DePARTAMENTO DE AUtOMÁTICA, IngENIERÍA ElÉCTRICA Y ELECTRÓNICA E INFORMÁTICA INDUSTRIAL

ESCUELA TÉCNICA SUPERIOR DE INGENIEROS INDUSTRIALES CENTRO DE ELECTRÓNICA INDUSTRIAL

\title{
Architecture and methodology for automated development of evolvable and reconfigurable hardware applications
}

Autor: Javier Mora de Sambricio

Máster en Electrónica Industrial por la Universidad Politécnica de Madrid

Director: Eduardo de la Torre Arnanz

Doctor Ingeniero Industrial por la Universidad Politécnica de Madrid 



\title{
Tribunal
}

Tribunal nombrado por el Excmo. y Magfco. Sr. Rector de la Universidad Politécnica de Madrid, el día de de 20

\author{
Presidenta: Marisa López Vallejo \\ Vocales: Lukáš Sekanina \\ Juan Antonio Gómez Pulido \\ Juan José Rodríguez Andina
}

Secretario: José Andrés Otero Marnotes

\section{Suplentes:}

Realizado el acto de lectura y defensa de la Tesis Doctoral el día de de 20 en la Escuela Técnica Superior de Ingenieros Industriales de la Universidad Politécnica de Madrid.

Calificación:

EL PRESIDENTE

LOS VOCALES

EL SECRETARIO 



\section{Acknowledgments}

To Edu, my supervisor; and my professors and colleagues, Tere, Jorge, Yago, Andrés, Alfonso, Arturo, Rubén, Juan, Ángel, and Txema; for putting up with me for all these years.

To Diana Göhringer and Michael Hübner for hosting me in the RuhrUniversität Bochum for 3 months; and Lester, Osvaldo, Philipp, Jones, Florian, and all other colleagues from there for being so friendly and welcoming.

To Lukáš Sekanina and Roland Dobai, for their invaluable collaboration.

To my parents, siblings, relatives, and friends, for their insistence and continued support, and to D. Francisco Silva for believing in me.

And finally, to the Universidad Politécnica de Madrid and the FPI grant program from the Spanish Ministry of Economy and Competitiveness for this opportunity. 



\section{Resumen en español}

This is the abstract in Spanish. For an English version of this abstract, please refer to page xi.

Uno de los retos en el ámbito del diseño de circuitos digitales es la necesidad de desarrollar sistemas que sean capaces de adaptarse a condiciones arbitrarias (y a menudo desconocidas) en tiempo de ejecución. Esto puede solucionarse en cierta medida dotando al circuito de varios modos de funcionamiento, o incluso de una funcionalidad programable, como es el caso de los microcontroladores o los procesadores digitales de señales; sin embargo, esta solución carece de autonomía (ya que el circuito necesita ser diseñado o programado explícitamente para tal propósito) y podría no ser lo bastante versátil como para cubrir todos los casos posibles.

El hardware evolutivo (EHW) proporciona una metodología para el diseño de circuitos digitales no convencional concebida para solventar esta dificultad. En EHW, la metodología de diseño cambia: en lugar de diseñar un circuito con conocimiento del problema a resolver, se «entrena» un circuito proveyendo un ejemplo de dicho problema junto con la solución que se desea. Para dicho entrenamiento se emplea un algoritmo evolutivo (EA). Los EAs son algoritmos metaheurísticos de optimización que tratan de hallar una solución mediante un proceso iterativo de prueba y error, realizando pequeñas modificaciones a una solución candidata de manera que su comportamiento en ciertas condiciones vaya mejorando incrementalmente, hasta dar con una solución suficientemente buena. En el caso del EHW, este algoritmo se emplearía para modificar partes aleatorias de un circuito hasta que su respuesta a cierta entrada se aproxime lo bastante a la salida deseada. Esto permite diseñar automáticamente circuitos para problemas que se desconoce cómo solucionar, pero para los cuales se conoce un ejemplo de "problema de entrenamiento" y la solución esperada para ese ejemplo. Una vez se ha entrenado el circuito para dicho ejemplo, éste seguramente sea capaz de procesar otros casos del mismo problema para los cuales no se conoce la salida ideal.

El EHW tiene aplicaciones en una gran variedad de campos, ya sea porque las condiciones de trabajo cambien con el tiempo, porque el problema no se conoce, o porque el sistema trabaja en un entorno hostil que cause la degradación paulatina del hardware (en este último caso, el EHW puede ser 
útil al adaptarse a los fallos del hardware según van apareciendo). Entre los ejemplos de aplicaciones del EHW se incluyen el filtrado de imagen y vídeo, clasificación de imágenes, reconocimiento de patrones, control de miembros protésicos, aplicaciones para satélites...

En el mundo del diseño de circuitos digitales se emplean comúnmente las llamadas matrices de puertas programables o FPGAs, que son circuitos integrados que contienen un gran número de elementos lógicos e interconexiones configurables que permiten la implementación de circuitos digitales arbitrarios, por lo que son una herramienta de gran utilidad para el prototipado de sistemas digitales. Asimismo, las FPGAs se usan para aplicaciones comerciales con bajos volúmenes de producción en las que desarrollar y fabricar un circuito integrado para aplicaciones específicas (ASIC) resultaría muy costoso. Sin embargo, éste no es el único uso de las FPGAs: dado que estos dispositivos (en su mayoría) se pueden reconfigurar tantas veces como se desee, también son útiles en aplicaciones en las que se precise de múltiples circuitos distintos para ser usado uno cada vez; en este caso puede emplearse una única FPGA en la que se cargue un circuito u otro según se necesite.

Ciertas FPGAs van más allá proveyendo la capacidad de realizar reconfiguración parcial dinámica (DPR), esto es, la capacidad de modificar una porción del circuito configurado mientras el resto funciona normalmente, sin necesidad de detener su funcionamiento. Esto es muy provechoso para la implementación de EHW, ya que puede hacerse un diseño que conste de varios bloques funcionales cuya funcionalidad se cambie mediante DPR, permitiendo generar configuraciones de circuitos arbitrarias que pueden modificarse empleando un EA. Además, la capacidad de reconfigurar una fracción del circuito mientras el resto sigue trabajando permite implementar un sistema completamente autónomo en la FPGA que ejecute EA, pruebe soluciones candidatas y finalmente use la solución optimizada para procesar datos provistos externamente.

El trabajo desarrollado en esta tesis doctoral tiene como antecedente el proyecto fin de carrera del autor en el Centro de Electrónica Industrial (CEI) de la Universidad Politécnica de Madrid (UPM). Este proyecto surgió como un trabajo de integración de los resultados parciales de las tesis de dos estudiantes de doctorado, Andrés Otero y Rubén Salvador. La tesis de Otero exploraba distintas metodologías para la implementación de DPR de grano fino, desde un motor de reconfiguración acelerado en hardware hasta una metodología de diseño para arquitecturas reconfigurables parcialmente. La tesis de Salvador, por otro lado, estudiaba distintos planteamientos para sistemas de EHW autoadaptativos; entre otras cosas, proponía el uso de una arquitectura de array sistólico como la base para una plataforma de EHW. El 
proyecto consistió en el desarrollo de una aplicación hardware a modo de prueba de concepto que integrara la metodología de reconfiguración de Otero con la arquitectura de array sistólico de Salvador para implementar un filtro de imágenes evolutivo. Este proyecto sirvió como demostración práctica de las metodologías propuestas en estas dos tesis.

Sin embargo, durante el desarrollo de dicho proyecto se hizo patente que, a diferencia del desarrollo de hardware convencional en el que los módulos pueden ser fácilmente parametrizados y reutilizados y son mayormente independientes de la plataforma, la metodología requerida para la implementación de EHW basado en DPR era bastante complicada, y requería ser repetida cada vez que se deseara realizar una nueva implementación. Esto suponía un obstáculo importante para la generalización de este paradigma ya que, si bien este proyecto mostraba su viabilidad y uso práctico empleando una aplicación específica, su extensión a nuevas aplicaciones requeriría repetir una gran parte del trabajo. Por esta razón, el autor determinó que era crucial generalizad y automatizar esta metodología de diseño.

Esta tesis ha establecido como objetivo principal la generalización de esta metodología de diseño y arquitectura de EHW orientadas a DPR con el fin de hacer su utilización en proyectos futuros lo más sencilla posible. Esto se ha abordado desde distintos flancos:

- Reemplazando la arquitectura específica para una única aplicación por un conjunto de módulos reutilizables altamente parametrizados.

- Proponiendo y siguiendo una metodología de diseño que automatice lo más posible la generación de sistemas de EHW.

- Desarrollando herramientas auxiliares para la generación de sistemas DPR.

- Explorando posibles aplicaciones que puedan beneficiarse de este paradigma, estudiando los requisitos que deban tenerse en cuenta a la hora de adaptar la arquitectura hardware a dichas aplicaciones.

- Optimizando la arquitectura hardware en la medida de lo posible, con el fin de que sea adecuada aún en tareas muy exigentes.

- Demostrando la idoneidad de la arquitectura de array sistólico propuesta por Salvador para EHW frente a alternativas habituales tales como la programación genética cartesiana.

Las aportaciones principales de esta tesis pueden resumirse como sigue: 
- Una metodología para la implementación de hardware reconfigurable dinámicamente para aplicaciones de EHW.

- Una serie de módulos hardware de altas prestaciones fuertemente parametrizados para la implementación de EHW basado en DPR, entre los que se incluyen un módulo de procesamiento de datos basado en un array sistólico y un motor de reconfiguración de grano fino.

- Un análisis minucioso de las prestaciones de los módulos desarrollados que muestra su rendimiento para distintos casos de uso, estudiando su escalabilidad, comparando implementaciones alternativas y explorando distintas posibilidades para acelerar su evolución.

- Una serie de herramientas de software auxiliares para la elaboración de EHW y hardware reconfigurable dinámicamente, entre las que se incluyen herramientas de extracción de bitstreams parciales para FPGA, modelos de simulación e implementaciones optimizadas de EA.

- Varias aplicaciones demostrativas basadas en esta metodología y módulos hardware.

Además, durante el desarrollo de esta tesis se han publicado tres artículos en revistas científicas JCR y numerosos artículos en conferencias.

Esta tesis ha sido subvencionada por el programa de ayudas de formación de personal investigador (FPI) del Ministerio de Economía y Competitividad de España (referencia de la ayuda BES-2012-060459), asociada al proyecto DREAMS (TEC2011-28666-C04) y su continuación, el proyecto REBECCA (TEC2014-58036-C4-2-R). 


\section{Abstract}

One of the challenges in the field of digital circuit design is the need to develop systems that will be able to adapt to arbitrary—and often unknownconditions at run time. This can be solved to a certain degree by making the circuit have multiple modes of operation, or even a programmable functionality, as is the case for microcontrollers and digital signal processors; however, this solution lacks autonomy-it needs to be explicitly designed or programmed for that purpose-and may not be versatile enough to cover all possible problems.

Evolvable hardware (EHW) provides an unconventional methodology for the design of digital circuits that was conceived to overcome this difficulty. In EHW, the design methodology changes: rather than designing a circuit with knowledge of the problem that needs to be solved, a circuit is "trained" by providing an example problem and the desired solution. For this training, an evolutionary algorithm (EA) is employed. EAs are metaheuristic optimization algorithms that attempt to find a solution through an incremental trial-anderror iterative process, making small modifications to a candidate solution so that its behavior under a certain problem is improved incrementally, until a good enough solution has been obtained. In the case of EHW, this algorithm would modify random parts of a circuit until its response to a certain input is close enough to the desired output. This permits the automatic design of circuits for problems whose general solution is unknown, but for which it is known what the solution for a specific "training problem" should be. Once the circuit has been trained for that example problem, it will ideally be able to process similar instances of the same problem for which the ideal output is not known.

EHW has applications in a wide variety of fields, whether because the working conditions change over time, the problem is unknown, or because the system works in a hostile environment that causes the degradation of the hardware-in this case, EHW can help by adapting to hardware faults as they appear. Example applications include image and video filtering, image classification, pattern recognition, prosthetic limb control, satellite applications...

In the field of digital circuit design, it is common to use field-programmable gate arrays (FPGAs). These are integrated circuits containing a large amount 
of configurable logic elements and interconnections that allow implementing arbitrary digital circuits, and thus are a helpful tool for the prototyping of digital systems. Moreover, FPGAs may be used for commercial applications with low production levels when developing and manufacturing an application-specific integrated circuit (ASIC) would be too costly. But this is not the only benefit of FPGAs: since these devices can be reconfigured on demand (in most cases), they are also useful in applications where multiple different circuits may be needed at different times; in this case, a single FPGA may be used and the required circuit would be loaded each time it is needed.

Some FPGAs go one step further and provide the ability to perform dynamic partial reconfiguration (DPR), this is, the ability to modify a fragment of its circuit configuration while the rest of it operates normally, without halting its operation. This is very convenient for the implementation of EHW, since a design can be made that consists of multiple functional blocks whose functionality is changed via DPR, thus permitting to generate arbitrary circuit configurations that can be modified using an EA. Furthermore, the ability to reconfigure a fraction of the circuit while the rest of it operates normally allows implementing a completely autonomous system on the FPGA that executes the EA, tests candidate solutions, and eventually uses the optimized solution for processing data provided externally.

The precursor of the work carried out in this Ph.D. thesis was the author's proyecto fin de carrera (B.Sc./M.Sc. final project) in the Centre of Industrial Electronics (CEI) of Universidad Politécnica de Madrid (UPM). This project was born as an integration work between the partial thesis results of two Ph.D. students, Andrés Otero and Rubén Salvador. Otero's thesis explored different methodologies for the implementation of fine-grain DPR, from a hardware-accelerated reconfiguration engine to a design methodology for partially reconfigurable architectures. Salvador's thesis, on the other hand, studied different approaches for self-adapting EHW systems; among other things, it proposed the use of a systolic array architecture as the base for an EHW platform. The project consisted in the development of a proof-of-concept hardware application integrating Otero's reconfiguration methodology with Salvador's systolic array architecture to implement an evolvable image filter. This project served as a demonstration of the methodologies proposed in these theses.

However, during the development of the aforementioned project, it became apparent that, unlike regular hardware development where modules can be easily parameterized and reused and are rather platform-independent, the methodology required for the implementation of DPR-based EHW was rather difficult, and needed to be repeated on a per-implementation basis. This 
posed an important obstacle for the generalization of this paradigm since, although this project demonstrated its feasibility and practical use using a specific application, extending it to new applications would require repeating most of the work. For this reason, the author found that the generalization and automation of this design methodology was crucial.

This thesis has set the main goal of generalizing this DPR-oriented design methodology and EHW architecture in order to make its use in future projects as straightforward as possible. This has been tackled from several angles:

- Replacing the application-specific architecture design with a set of highly parameterized reusable modules.

- Proposing and following a design methodology that automates the generation of EHW systems to the extent possible.

- Developing tools to assist in the generation of DPR systems.

- Exploring possible applications that may benefit from this paradigm, studying the requirements that should be taken into account to adapt the hardware architecture to them.

- Optimizing the hardware architecture as much as possible in order to promote its suitability for demanding tasks.

- Proving the suitability of the systolic array architecture proposed by Salvador for EHW over commonly used alternatives such as Cartesian genetic programming.

The main contributions of this thesis can be summarized as follows:

- A methodology for the implementation of dynamically reconfigurable hardware for EHW applications.

- A series of highly parameterized and efficient hardware modules for the implementation of DPR-based EHW, including a data processing module based on a systolic array and a fine-grain reconfiguration engine.

- A thorough analysis of the capabilities of the developed modules showing its performance under several use cases, studying its scalability, comparing alternative implementations, and exploring different possibilities to accelerate its evolution. 
- A series of software tools to assist in the elaboration of EHW and dynamically reconfigurable hardware, including partial FPGA bitstream extraction tools, simulation models, and optimized EA implementations.

- Several demonstrative applications based on this methodology and hardware modules.

In addition, three JCR journal articles and numerous conference papers have been published during the development of this thesis.

This thesis has been sponsored by the FPI grant program of the Spanish Ministry of Economy and Competitiveness (grant number BES-2012-060459), associated to project DREAMS (TEC2011-28666-C04) and its continuation, project REBECCA (TEC2014-58036-C4-2-R). 


\section{Contents}

Acknowledgments $\quad$ v

Resumen en español vii

Abstract $\quad$ xi

$\begin{array}{ll}\text { Contents } & \text { Xv }\end{array}$

List of Figures $\quad$ xix

List of Tables $\quad$ xxiii

1 Introduction 1

1.1 A brief introduction to the context of this thesis $\ldots \ldots \ldots$

1.2 Thesis motivation . . . . . . . . . . . . . 3

1.3 Thesis objective and main goals . . . . . . . . . . . . . 4

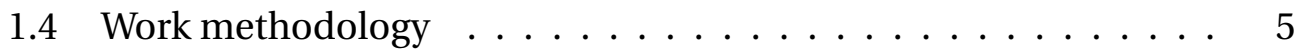

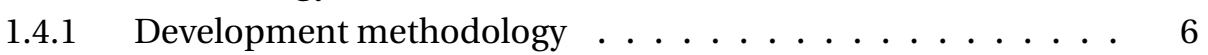

1.4.2 Methodology for the design of experimental tests . . . . . 6

1.4.3 Employed hardware and software tools . . . . . . . . . . 9

1.5 Thesis structure and organization . . . . . . . . . . . . 10

1.5.1 Structure and hierarchy of the thesis . . . . . . . . 10

1.5.2 Organization of the rest of the thesis $\ldots \ldots \ldots 10$

2 Technical background $\quad 13$

2.1 Introduction to evolvable hardware . . . . . . . . . . . . . . . 13

2.2 Implementation techniques for EHW systems . . . . . . . . . . 16

2.2.1 Evolutionary algorithms . . . . . . . . . . . . 16

2.2.2 PE interconnection topologies . . . . . . . . . . . . 22

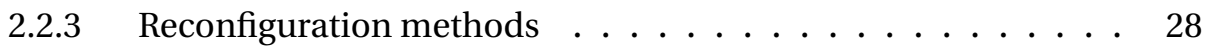

2.3 State of the Art . . . . . . . . . . . . . . . . . 32

2.4 Research opportunities . . . . . . . . . . . . . . . . 34 
3 Preliminary development of an EHW system 37

3.1 Description of the preliminary system . . . . . . . . . . . . 38

3.1.1 Systolic array characteristics . . . . . . . . . . . . 40

3.1.2 Reconfiguration methodology . . . . . . . . . . . . . . 41

3.1.3 Preliminary evolutionary algorithm . . . . . . . . . . . 47

3.2 System analysis and experimental results . . . . . . . . . . . 50

3.2.1 System characterization . . . . . . . . . . . . . . 50

3.2 .2 Evolution results $\ldots \ldots \ldots \ldots \ldots$

3.3 Assessment of the validity of the solution . . . . . . . . . . . 55

3.3 .1 Generalizability . . . . . . . . . . . . . . 56

$3.3 .2 \quad$ Adaptivity . . . . . . . . . . . . . . . . 57

3.3.3 Fault tolerance . . . . . . . . . . . . . . . . 6 61

3.3 .4 Scalability . . . . . . . . . . . . . . 63

3.3.5 Real-time capabilities . . . . . . . . . . . . . . 64

3.4 Derived applications . . . . . . . . . . . . . . . 65

3.4.1 CPU and GPU implementations . . . . . . . . . . . . . 65

3.4.2 Realistic noise effects and extension to color images . . . . . . 65

3.4.3 Exploiting scalability for increased fault tolerance . . . . . . 66

3.4.4 Learning by imitation . . . . . . . . . . . . . . . . 66

3.4.5 Real-time scalable demo . . . . . . . . . . . . . . 66

3.5 Problems and motivation for changes . . . . . . . . . . . . 68

3.5 .1 Integration $\ldots \ldots \ldots \ldots \ldots \ldots$

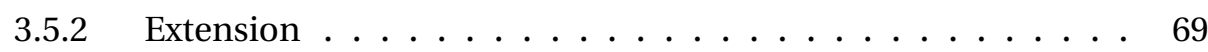

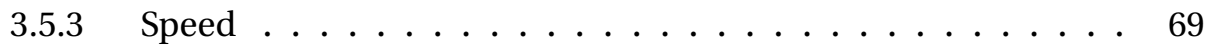

3.5.4 Real-time processing and larger video formats . . . . . . . . 69

3.5.5 Technology transition . . . . . . . . . . . . 70

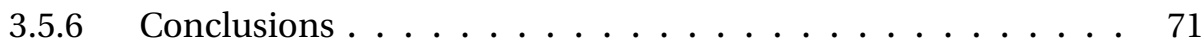

4 Reconfiguration techniques $\quad 73$

4.1 Generic reconfiguration engine . . . . . . . . . . . 73

4.1.1 Motivation and previous work . . . . . . . . . . 74

4.1.2 Description of the chosen solution $\ldots \ldots \ldots \ldots$

4.1 .3 Implementation . . . . . . . . . . . . . . 77

4.2 Partial bitstream extraction methodology . . . . . . . . . . 80

4.2 .1 Motivation . . . . . . . . . . . . . . . 80

4.2 .2 Framework description . . . . . . . . . . . . . . 82

4.2 .3 Derived applications $\ldots \ldots \ldots \ldots \ldots$

5 Architecture and HW design methodology 87 
5.1 LUT-based reconfiguration . . . . . . . . . . . . . . . 88

5.1.1 Understanding Virtex-5 CLBs . . . . . . . . . . . . . . . 89

5.1.2 Development of LUT-based PEs . . . . . . . . . . . . . . . 95

5.1.3 Extending to input and output elements . . . . . . . . . 100

5.1.4 Placement constraints . . . . . . . . . . . . . . 103

5.1.5 Reconfiguring a LUT-based design $\ldots \ldots \ldots \ldots$

5.1.6 Analysis of the solution and experimental results . . . . . . . . 109

5.2 Comparison of CGP and SA . . . . . . . . . . . . . . . 112

5.2 .1 Implementation details . . . . . . . . . . . . . 113

5.2 .2 Resource usage analysis . . . . . . . . . . . . . . 117

5.2 .3 Evolution results . . . . . . . . . . . . . . . . . . 119

5.2.4 Improving the behavior of SA with scalability . . . . . . . 120

5.2 .5 Other considerations . . . . . . . . . . . . . 127

5.2 .6 Conclusions . . . . . . . . . . . . . . . . . 128

5.3 Redesigning the system . . . . . . . . . . . . . . . . . 129

5.3 .1 Design considerations . . . . . . . . . . . 130

5.3.2 Separation of the design into different clock domains . . . . 131

5.3.3 Simplifying the design for a better performance . . . . . . . 132

5.3.4 Accelerating the evolution using multiple arrays in parallel . . 138

5.3.5 Analysis of the solution and experimental results . . . . . . . . 139

5.3 .6 Conclusions . . . . . . . . . . . . . . . . . . . . . 142

5.4 Moving to a dataflow paradigm . . . . . . . . . . . . . 143

5.4.1 Towards a dataflow-oriented paradigm . . . . . . . . . . 144

5.4.2 Choosing an interconnection interface: AXI4-Stream $\ldots \ldots 147$

5.4.3 Porting the system to the new paradigm . . . . . . . . . 152

5.4 .4 Conclusions . . . . . . . . . . . . . . . . 157

6 Improvements on the evolution techniques 159

6.1 Accelerating the convergence of the EA . . . . . . . . . . 159

6.1.1 Specification of the test case and initial results . . . . . . 160

6.1.2 Finding the optimal mutation rate . . . . . . . . . . . . . 162

6.1.3 Effect of the offspring size . . . . . . . . . . . . . . 164

6.1.4 Increasing the diversity to avoid the effect of local optima . . . 165

6.1.5 Promoting good evolutions . . . . . . . . . . 167

6.1.6 Restricting mutations to a single column . . . . . . . . . 170

6.1.7 Evaluation of results and conclusions . . . . . . . . . 170

6.2 Exploiting parallelism . . . . . . . . . . . . . 171

6.2.1 Analysis of the parallelizability of the system . . . . . . . 171

6.2 .2 Improved scheduling . . . . . . . . . . . . . 173 
6.2.3 Evaluation of results and conclusions . . . . . . . . . 177

$\begin{array}{llr}7 & \text { Exploration of applications } & 179\end{array}$

7.1 Noise-agnostic evolution . . . . . . . . . . . . . . . . 180

7.1.1 Usage of a noisy image as a reference . . . . . . . . . . . 181

7.1.2 Usage of two consecutive noisy frames as training data set . . 184

7.1 .3 Triggering evolution . . . . . . . . . . . . . . 188

7.1.4 Conclusions . . . . . . . . . . . . . . . . . 188

7.2 Extending the paradigm to different problems . . . . . . . . . 189

7.2.1 Exploring applications other than substitutive noise filtering $\quad 190$

7.2.2 Generalization of the reconfiguration engine . . . . . . . 193

7.2.3 Increasing the size of the sliding window $\ldots \ldots \ldots$. . . . 198

7.2.4 Improving the PE library . . . . . . . . . . . . . . . . . . 199

7.2 .5 Conclusions . . . . . . . . . . . . . . . . 208

7.3 Example dataflow-based application . . . . . . . . . . . . . 209

7.3.1 Description of the video processing system . . . . . . . . 210

7.3.2 Description of the different video blocks . . . . . . . . . 211

7.3.3 Analysis of the resulting system and conclusions . . . . . . . . 212

8 Conclusions and future lines $\quad 215$

8.1 Conclusions of the thesis . . . . . . . . . . . . . . . . 215

8.2 Summary of main contributions . . . . . . . . . . . . . . 217

8.2.1 Methodological contributions . . . . . . . . . . . 217

8.2.2 Analytical contributions . . . . . . . . . . . . . . 219

8.2.3 Developed hardware modules . . . . . . . . . . . . . 220

8.2.4 Developed software tools . . . . . . . . . . . . . . . 222

8.2.5 Summary of improvements on the HW platform . . . . . . . 222

8.3 Impact of the thesis . . . . . . . . . . . . . . . . . . 224

8.3.1 Publications and dissemination . . . . . . . . . . . . . 224

8.3.2 Related projects and research lines . . . . . . . . . . 228

8.3.3 Co-supervised works . . . . . . . . . . . . . . . . . 229

8.4 Future work . . . . . . . . . . . . . . . . . . 231

8.4.1 Experimental analysis and exploitation of the architecture . . 232

8.4.2 Evolutionary algorithm . . . . . . . . . . . . . 233

8.4.3 Architecture . . . . . . . . . . . . . . 233

8.4.4 Recofiguration methodology and tools . . . . . . . . . 235

8.4.5 Applications and future projects . . . . . . . . . 236

$\begin{array}{ll}\text { Bibliography } & 237\end{array}$ 


\section{List of Figures}

1-1 Histogram and box plot of a fitness sample . . . . . . . . 7

1-2 Box plot of two samples with different sample sizes $\ldots \ldots . . .8$

2-1 Fitness landscape examples . . . . . . . . . . . . . . . 18

2-2 Some examples of crossover . . . . . . . . . . . . . . . 19

2-3 Mutation ...................... 19

2-4 Tree representation of an algebraic expression . . . . . . . . 21

2-5 Crossover mechanism used in GP . . . . . . . . . . . . . . . 21

2-6 "NEWS" two-dimensional grid topology . . . . . . . . . . . . . . . . . 23

2-7 Artificial neural network . . . . . . . . . . . . . . . . 23

2-8 Block-based neural network . . . . . . . . . . . . 25

2-9 Cartesian genetic programming topology . . . . . . . . . . 26

2-10 Three examples of systolic array topologies . . . . . . . . . . . 27

2-11 Reconfiguration method used by virtual reconfigurable circuits . 29

2-12 Reconfiguration method used in dynamic partial reconfiguration 30

2-13 A simple 2-input look-up table implementing the XOR function . 31

3-1 Sliding window . . . . . . . . . . . . . . . . . . . 39

3-2 Experimental PSNR results obtained using SAE and SSE . . . . 40

3-3 4x4 systolic array and detail of each PE . . . . . . . . . . 40

3-4 8-bit bus macro as seen in FPGA Editor . . . . . . . . . . . . . 44

3-5 Bus macro based SA . . . . . . . . . . . . . . . . . . 45

3-6 Structure of the FPGA configuration memory . . . . . . . . . . 46

3-7 Example set of training images . . . . . . . . . . . . . . 49

3-8 Set of training images used for the tests . . . . . . . . . 53

3-9 Distribution the results for different mutation rates . . . . . . . 53

3-10 Progression of the fitness value during the evolution . . . . . . . . 54

3-11 Some of the results obtained with the evolved filters . . . . . . . 55

3-12 Example of overfitting . . . . . . . . . . . . . . . . 57

3-13 Results of filters trained with an image when applied to others . . 58

3-14 Different types of noise . . . . . . . . . . . . . . . . 59

3-15 SAE obtained when filtering images with filters trained for different noise levels . . . . . . . . . . . . . . . . 60

3-16 Result of filtering images with filters trained for different noise

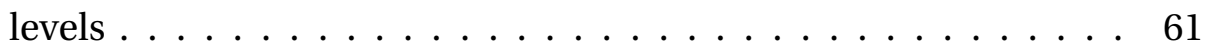


3-17 Output selector . . . . . . . . . . . . . . . . . 62

3-18 The latency of the solution depends on the predominant data path 63

3-19 Computing of fitnesses corresponding to different latencies using multiple fitness computing units . . . . . . . . . . . . 63

3-20 Reduction of 9 input channels to 3 combined with additional delay 64

3-21 Screenshot of the demo . . . . . . . . . . . . . . . . 67

4-1 Signal interface of the generic reconfiguration module . . . . . 77

4-2 Two-phase handshake . . . . . . . . . . . . . 78

4-3 Example use of the command-line extraction tool . . . . . . . . 83

4-4 Partial bitstream extraction GUI . . . . . . . . . . . . . . . . 84

4-5 Visualization of the bitstream content . . . . . . . . . . . 85

4-6 Example use of the interactive application . . . . . . . . . . . 86

5-1 Arrangement of slices within a CLB . . . . . . . . . . . . 90

5-2 Structure of a Virtex-5 SLICEL . . . . . . . . . . . . . . . . . . 91

5-3 Implementation of a Virtex-5 6-input LUT . . . . . . . . . . . . . . 92

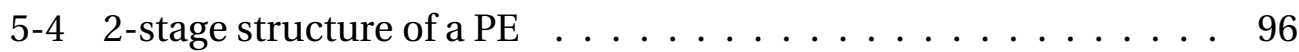

5-5 Fragment of a LUT-based PE, first version . . . . . . . . . . . . 98

5-6 Fragment of a LUT-based PE, improved version . . . . . . . . . . . 99

5-7 Fragment of a LUT-based input selector . . . . . . . . . . . . . . 101

$5-8$ Pipelining of the input selectors . . . . . . . . . . . . . . 101

$5-9$ Diagram of the output selector . . . . . . . . . . . . . 102

5-10 Pipelined data propagation through the SA in separate wavefronts 102

5-11 Specification of the SA position within the FPGA . . . . . . . . 105

5-12 Detail of the interconnections between PEs . . . . . . . . . . 110

5-13 Screen capture of Xilinx FPGA Editor showing a $24 \times 24$ array . . . 111

5-14 Training images used in the evolution . . . . . . . . . . 117

5-15 Comparison of resource usage versus number of PEs for both SA and CGP . . . . . . . . . . . . . . . . . . . 118

5-16 Fitness for different sizes of SA and CGP . . . . . . . . . . . . . 119

5-17 Fitness and LUT usage for different sizes of SA and CGP . . . . . . 120

5-18 Result of having an evolution four times longer . . . . . . . . . 121

5-19 Evolution results growing from an initial SA size of $10 \times 10 \ldots 122$

5-20 Possible routing of a primary input to an arbitrary PE . . . . . . 123

5-21 New PEs: cross and diverge . . . . . . . . . . . . . . . . . . 123

5-22 Two-output PE architecture . . . . . . . . . . . . . . . 124

5-23 Routing a primary input to an arbitrary PE with the new PEs . . . 124

5-24 Results obtained with the new PEs . . . . . . . . . . . . . . 125

5-25 Functional diagram of a bypassable PE . . . . . . . . . . . . . 125

5-26 Results obtained with the bypassable PEs . . . . . . . . . . 126 
5-27 Clock domain division of the system . . . . . . . . . . . . . . . 132

5-28 Window behavior at image border . . . . . . . . . . . 134

5-29 5×1 SA with inputs directly connected to the PEs . . . . . . . . 135

5 -30 Effect of pipelining on the fitness . . . . . . . . . . 136

5-31 Parallelized fitness computing unit . . . . . . . . . . . . 137

5-32 Different interconnection possibilities . . . . . . . . . . . . . 144

5-33 Diagram of a single SA-based processing module . . . . . . . . 145

5-34 Implementation of a Sobel filter using static and dynamic approaches . . . . . . . . . . . . . 146

5-35 Screenshot of Vivado IP Integrator displaying a block design . . . 148

5-36 Valid-ready handshake . . . . . . . . . . . . . . . . . . . 149

5-37 AXI4-Stream Video bus for an 8-bit video signal . . . . . . . . 150

5-38 Arrangement of the two PE stages in Virtex-5 and 7 Series implementations . . . . . . . . . . . . . . 153

5-39 Fragment of a dataflow-oriented PE . . . . . . . . . . . . . 154

5-40 Variable width window generator implemented as a circular buffer155

6-1 Progress of the fitness along the evolution . . . . . . . . . . . 161

6-2 Results obtained for different mutation rates . . . . . . . . . . 163

6-3 Results for a (1+8)-EA and a (1+1)-EA . . . . . . . . . . . . . 164

6-4 Median fitness of the best of $n$ evolutions . . . . . . . . . . 166

6-5 Comparison of the original (1+8)-EA with the best of $12(1+1)$-EA 167

6-6 Comparison of (1+8)-EA, best of $12(1+1)$-EA, and $12(1+1)$-EA with exchanges . . . . . . . . . . . . . . . 169

6-7 EA performance at different exchange intervals . . . . . . . . . 169

6-8 Results of mutating any column vs a single column . . . . . . 170

6-9 Number of evaluations per second achieved with multiple SAs in parallel. . . . . . . . . . . . . . . . . . 173

6-10 Comparison of both scheduling strategies for a 12 SA system . . . 174

6-11 Comparison of the previous parallelization approach with the half-and-half scheduling . . . . . . . . . . . . . 175

7-1 Some frames from the Foreman sequence . . . . . . . . . . . . 181

7-2 Evolution performed with a noisy image as a reference . . . . . 182

7-3 Evolution results using a noisy image as reference . . . . . . . 183

7-4 Evolution results using two consecutive frames . . . . . . . . . . 185

7-5 Correlation between SAE of noisy and noise-free frames . . . 186

7-6 Evolution results using two similar consecutive frames . . . . . 187

7-7 Input and reference images for different problems . . . . . . . . 191

7-8 Evolution results for the five analyzed problems . . . . . . . . . 192

$7-9$ Output of some of the evolved filters . . . . . . . . . . . 192 
7-10 Example mapping from hierarchical indexes to PBS address . . . 196

7-11 Comparison of evolution results with $3 \times 3$ and $5 \times 5$ windows . . . 199

7-12 Evolution results with the extended PE library . . . . . . . . . 202

7-13 Evolution results with the PE library optimized for S\&P . . . . . 205

7-14 Evolution results with a PE library optimized for each problem . 206

7-15 Evolution results with the proposed PE library . . . . . . . . . 208

7-16 Block diagram of the dataflow demonstration application . . . 210

7-17 Result of applying a Sobel filter to an image with and without noise filtering . . . . . . . . . . . . . . 214

8-1 Possible implementation of a $6 \times 9$ window selector . . . . . . . . 234 


\section{List of Tables}

2-1 State of the Art . . . . . . . . . . . . . 32

3-1 Functions implemented by the PEs . . . . . . . . . . . . . . . . 42

3-2 PE library size with and without relocation $\ldots \ldots . \ldots 43$

3-3 Resource usage of the system . . . . . . . . . . . . 51

5-1 Distribution of the content of the 4 LUTs . . . . . . . . . . . . . . 93

$5-2$ Position of LUT entries in the bitstream . . . . . . . . . . 93

5-3 LUT entry usage with A2=0 . . . . . . . . . . . . . . . 95

5-4 Two-stage implementation of PE functions . . . . . . . . . . 97

5-5 Register description for the LUT-based PE reconfiguration peripheral . . . . . . . . . . . . . . . 108

5-6 Functions implemented by the PEs in CGP . . . . . . . . . . . . 115

5-7 Resource usage of CGP and SA at different sizes . . . . . . . 118

5-8 Resource usage of the EHW system . . . . . . . . . . . . . 140

5-9 Evolution time breakdown . . . . . . . . . . . . . . . . . . 142

6-1 Evolution time breakdown for a single evaluation $\ldots \ldots \ldots$

7-1 Example register description . . . . . . . . . . . . . . 196

$7-2$ List of proposed PE functions . . . . . . . . . . . . . . 201

7-3 Rank of each PE function for each problem and overall rank . . . 204

7-4 Proposed PE function library . . . . . . . . . . . . . . . . . 207

7-5 Resource usage of the video filtering system . . . . . . . . . . . 213

8-1 Progression of the resource usage and scalability . . . . . . . . . 223

8-2 Incremental improvements on the evolution time . . . . . . . . 223 



\section{Chapter}

\section{INTRODUCTION}

This chapter aims to introduce the reader to the context of this thesis, explaining its motivation, intent, and organization.

First, a brief introduction of the basic concepts covered in this thesis is presented in section 1.1 in order to provide some background context for the development of this thesis.

Then, a series of existing problems that this thesis aims to cover and that motivate its development are described in section 1.2. The main goals of the thesis, which aim to solve those problems, are listed in section 1.3, complete with references to the chapters and sections where each of these goals is approached.

Section 1.4 details the way of working followed in this thesis, from the research "philosophy" and development guidelines to the methodology used to obtain experimental results.

Finally, section 1.5 summarizes the organization of the thesis, briefly describing each chapter and highlighting the most important results and conclusions that have been obtained.

\subsection{A BRIEF INTRODUCTION TO THE CONTEXT OF THIS THESIS}

This thesis focuses on the study and development of digital applications based on a design paradigm known as evolvable hardware (EHW). This paradigm consists in the automated generation of ad hoc electronic circuits that are able to adapt to different tasks which may not be known at design time.

Typically, an EHW implementation will consist of a series of interconnected processing elements (PEs) which perform a specific operation, processing the 
data they receive at their input and transmitting the obtained result to either the circuit output or to other PEs. The set of specific processing functions implemented by each PE and the way PEs are interconnected constitute one possible configuration of an EHW system. The key feature of EHW is the possibility of selecting different possible configurations after the system has been designed.

The distinctive property of EHW which differentiates it from other adaptive systems is the use of an evolutionary algorithm (EA) to find a satisfactory configuration. An EA is an optimization algorithm inspired by biological evolution which finds solutions to a problem through an iterative process of trial and error. This process follows a random incremental search: on each iteration, new candidate solutions are obtained by randomly combining and modifying previously existing ones; then, the obtained solutions are compared so that better solutions are more prone to be selected in future iterations over worse ones. This comparison is normally performed by calculating a fitness value, which is a figure of merit that indicates the degree to which a solution conforms to the problem to solve.

Field-programmable gate arrays (FPGAs) provide an excellent platform to develop such digital circuit implementations, as they can be configured to implement different digital circuits. Furthermore, some FPGAs provide the possibility of performing dynamic partial reconfiguration (DPR), by which a fragment of the FPGA circuit can be reconfigured while the rest of it continues working. This allows implementing autonomous EHW systems that run entirely within the FPGA and do not require any other external tool (such as a PC) to generate, evaluate, and implement the evolved circuit.

This thesis stems from research lines in the field of EHW [Salvador'15] and DPR [Otero'14] that were developed in the Centre of Industrial Electronics at the Universidad Politécnica de Madrid, and is a continuation of the author's B.Sc./M.Sc. final project [Mora'11] and master thesis [Mora'13a], which revolved around the implementation of evolvable image filters based on DPR.

However, although several applications were developed demonstrating the possibilities of this paradigm [Otero'11b, Salvador'11b, Otero'12a, Mora'14, Mora'13b], all these were limited to a narrow range of use cases, focusing mainly in the implementation and study of those specific applications without a more global vision of the possibilities of such a paradigm, neglecting its potential for other applications. The main goal of this thesis is to study different aspects of the generalization of this problem to different use cases. 


\subsection{THESIS MOTIVATION}

As it has been said, the main problem with the design methodology used in previous work is the fact that it is focused on a single application, with no considerations of reusability and generalizability. Therefore, any plans to adapt this work to a different use case or platform would face an obstacle: they would require a considerable amount of work, rewriting most of the system and repeating all the steps that were necessary to produce an implementation adapted to the needs of the new application.

For example, something as simple as changing the size of the processing architecture in order to obtain higher processing capabilities requires manually calculating and specifying the positions of the reconfigurable region boundaries within the FPGA, synthesizing the system, and double-checking that the obtained system does not violate any requirement for DPR to be feasible; unfortunately, this process is not automated in any way and has to be done manually. Some more significant changes to the system, such as changing the mode of operation or adapting to arbitrarily large image sizessomething necessary in order to adapt this system to real-time video filtering applications-would require a complete rewrite of the system, which would be difficult given the high degree of specialization the developed modules have. Additionally, all the previous work has been targeted to a specific FPGA model, which may be considered obsolete as newer FPGA families and tools targeting these families are released.

Despite the aforementioned difficulties, several derived projects have emerged, exploring possibilities such as cascading multiple evolvable filters [Gallego'14, Polop'14], implementing a hardware-accelerated EA [Lanza'13], filtering large video formats [González'17] and different pixel depths, implementing noise detectors for realistic noise types [Niño'15], or filtering entirely different signals such as electroencephalograms [López'14]. However, most of these projects required a great amount of work to adapt the existing system to those applications, or were not actually implemented at all but only studied theoretically through simulations. Nevertheless, the emergence of these derived applications shows the utility and convenience of this kind of system, bringing to light the importance of improving them and simplifying their implementation.

Therefore, in order to explore the full potential of the system in scope (improving its performance, studying its scalability, exploring new applications, comparing its behavior to existing alternatives...) it becomes 
necessary to develop a series of methodologies and tools that simplify the elaboration of this kind of system, independently from the specific application in scope.

This thesis pursues to remove the barriers inherent to the development of EHW applications, opening the possibility of creating miscellaneous systems based on this approach, from applications that integrate the described module as one more element in a more complex system to implementations that reuse parts of it with the necessary modifications to adapt them to different tasks.

\subsection{THESIS OBJECTIVE AND MAIN GOALS}

The object of this thesis is to propose methodologies, supported by the required tools and architectures, to automate and simplify the generation of systems based on EHW and DPR.

The main goals that have been pursued in this thesis are described below. The sections in which each of these topics has been most prominently studied is indicated in parentheses.

To study methodologies to improve the evolvable processing system described in previous work. These improvements focus on three main aspects:

- Make the system evolve faster, which includes reducing the filtering time $(\S 5.3, \S 6.2$ ) and reconfiguration time ( $\$ 4.1)$, as well as improving the EA in order to reach acceptable solutions requiring fewer evaluations $(\S 6.1)$.

- Obtain better results by improving the array topology and functionality $(\$ 5.2, \S 7.2)$ in addition to the aforementioned improvements on the EA. This includes comparing the performance of different EHW topologies in use in the state of the art $(\$ 5.2)$.

- Make the system smaller in terms of resource usage ( $\$ 5.1, \S 5.2$ ), in order to promote its scalability $(\$ 5.2)$, parallelization $(\$ 6.2)$, and integration into other projects $(\$ 7.3)$. 
To generalize the paradigm applied in this system in order to expand its range of application. This includes:

- Ensure the system is useful for a variety of tasks and under different conditions $(\$ 7.1, \S 7.2)$.

- Identify potential applications that could benefit from this paradigm $(\S 7.2)$.

- Parameterize and modularize the system to adapt it to different needs $(\S 5.1, \S 5.3)$

- Make the system easy to integrate with other projects $(\S 5.4, \S 7.3)$.

- Ensure the paradigm can be used in a range of platforms, in order to prevent locking into a single one that may become outdated (\$ 5.4).

- Provide the necessary tools for different DPR-related projects (\$ 4.2).

To define a design methodology that can be used in future projects for developing similar or related systems based on dynamic partial reconfiguration. This design methodology focuses on the following points:

- Ease of adoption and integration $(\S 5.1, \S 5.3, \S 5.4, \S 7.2)$.

- Reusability (§4.1, §5.3, §5.4).

- Ease of improvement and extension (\$ 5.3, § 7.2).

\subsection{WORK METHODOLOGY}

This section describes the guidelines and premises that have been followed during the development of this thesis. 


\subsubsection{Development methodology}

The work described in this thesis has followed the premise that it should be generalizable. This means that all modules, tools, and methodologies developed have been elaborated with the idea that they should be easily adaptable to different situations rather than a specific task. This way, although the thesis revolves around a specific use case that has been used as the basis for most of the development, the results of this work are not limited to said application; rather, they can be applied to a wide range of potential applications and situations.

In order to achieve this generalizability, all developed tools and systems have been designed in a modular way, isolating each different functionality on a separate module to the maximum extent possible. This modular development approach aims at fulfilling two goals: to simplify the modification and improvement of different parts (since changes on a certain functionality will be mostly limited to a certain module, reducing the side effects and necessary work on other modules), and to promote the development of derived or extended utilities by facilitating the reusability of these modules.

The thought process followed on the majority of this thesis has consisted on the following three-part process: (i) devise possible use cases that may benefit from the paradigm and tools studied in this thesis, (ii) study how these challenges could be solved with the available means, and (iii) if the tools and methodologies are not suited for these problems, consider extending or generalizing them to support these use cases or facilitate their solution. With this process, the author intends to ensure that the work presented here be generalizable to a wide range of applications.

\subsubsection{Methodology for the design of experimental tests}

This thesis has put special emphasis in the accuracy and validity of experimental results. These are very important not only in the assessment of achievements made in this work but also in the decision of which development lines have yielded results worth researching: they are a tool to guide the progress of the work.

Each experiment performed in this thesis consists of a set of evolutionary runs that are performed on the system under test. In order to obtain statistically meaningful results, several evolutionary runs are executed using different initial random seeds, which will lead to different evolution results. These results will then be subject to a statistical analysis to assess the behavior of the system under test and compare it to different solutions. 
The figure of merit used in this thesis as a comparative metric between different solutions will be the fitness achieved at the end of each evolution. Since each experiment consists of several evolutionary runs, no single fitness value is obtained, but rather a distribution of them. As an example, Figure 1-1 shows a histogram representing the distribution of 1000 fitness values that were obtained in one of the experiments. As can be seen, these values do not follow a symmetric distribution, but a rather skewed one with heavy tails, which makes the mean (indicated with a cross on the box plot) significantly different from the median.

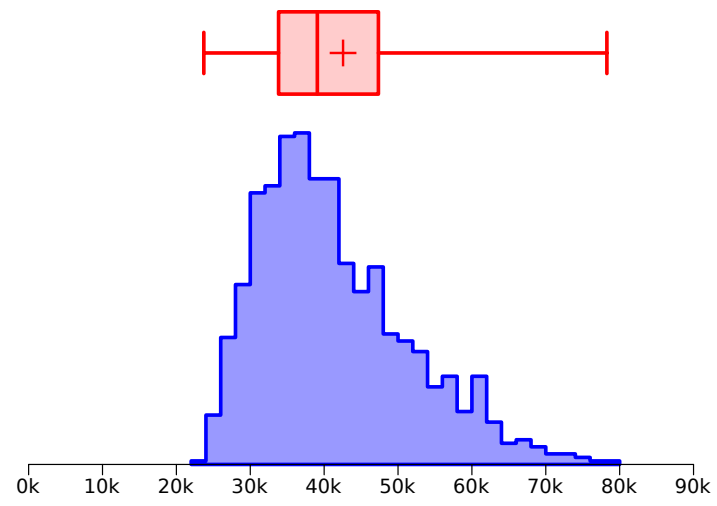

Figure 1-1: Histogram and box plot of a fitness sample

In order to make the statistics more robust to outliers and skewed distributions, this thesis makes heavy use of the median and quartiles when analyzing results. These are graphically represented in the form of box plots or, when intended to represent a continuously varying distribution (e.g. the progress of an evolution in time), as line graphs showing the median and quartiles. Since minimum and maximum of a sample have little statistical relevance, they are not shown in the latter graphs.

In order to ensure that the results are statistically significant, each experiment uses a large number of values, from 100 to 1000 depending on the required reliability. When, despite of the large sample sizes used, it is not clear whether or not two results should be considered different, statistical significance tests such as Student's t-test [Student'08] or Wilcoxon-MannWhitney test [Mann'47] are used.

Although using these many values may seem excessive, experience has shown that an excessively small number of tests can produce misleading results. This is illustrated in Figure 1-2. With a small sample size (10 values), samples 1 and 2 appear to be clearly different. Increasing the sample size 
to 30 still suggests that sample 2 has a much lower value than sample 1 overall. However, when the sample size is increased to 100 , the results no longer suggest a significant difference between distributions. Finally, a plot of 1000 values reveals that the distributions are actually pretty similar. In fact, both samples have been obtained from the same normal distribution with $\mu=0$ and $\sigma=1$.

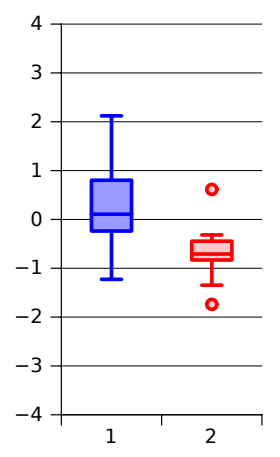

$n=10$

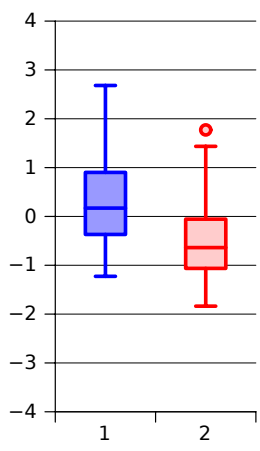

$n=30$

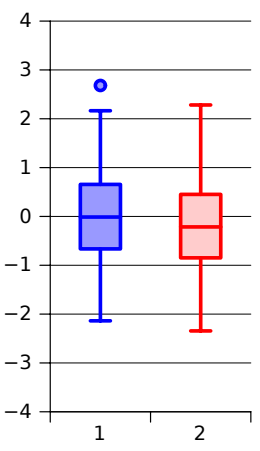

$n=100$

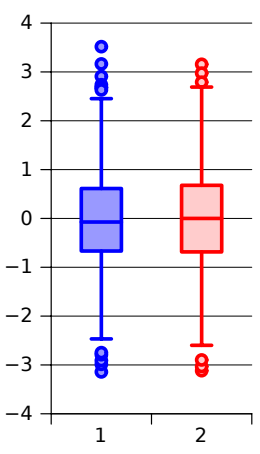

$n=1000$

Figure 1-2: Box plot of two samples obtained from the same normal distribution with different sample sizes $(n)$

All experimental tests elaborated in this thesis have been designed to be strictly repeatable, which is achieved by using a known random number generator and initial random seeds. This serves two purposes: to be able to repeat a specific run of an experiment (for example, to retrieve some additional data that had been discarded in the original experiment without repeating all of it), and to ensure that a specific implementation behaves exactly as expected, using an earlier version or a software simulation of its functional behavior as reference.

One of the benefits of evolvable hardware-its ability to overcome faultsbecomes a disadvantage when evaluating and characterizing such systems, since it can conceal underlying problems such as timing errors which can lead to unpredictable behavior. Thus, the assessment of the correct operation of a certain implementation shall be very strict, requiring the obtained results to be exactly as predicted in a simulated model. If the obtained result happens to be worse or better than the expected one, the implementation would be deemed incorrect and the reason for this discrepancy would be studied and mitigated. This approach ensures the repeatability of experiments and a predictable behavior.

Finally, it should be highlighted that all experimental tests aim at making fair comparisons of the metrics being evaluated; for example, comparing 
scalability in terms of resource usage rather than number of processing elements when these elements are significantly different in size, or measuring evolution length in terms of time rather than number of generations when measuring the performance of different EAs.

\subsubsection{Employed hardware and software tools}

The majority of the development and tests performed in this thesis have been elaborated using a Xilinx® University Program XUPV5-LX110T development. This board features a Xilinx Virtex®-5 LX110T, a high-performance FPGA with dynamic partial reconfiguration capabilities that can operate at frequencies of up to $550 \mathrm{MHz}$ [Xilinx-DS100]. The choice of this relatively old platform (released a decade ago) as the main FPGA used for development is motivated by the extensive work that had been previously performed targeting that FPGA as well as the detailed documentation on its reconfiguration procedures available. Nevertheless, newer platforms such as the Zynq-7000 are explored as well.

Hardware development (system, modules, and applications) has been performed using Xilinx ISE® Design Suite, which includes the Xilinx Platform Studio (XPS) embedded system design component, and the Software Development Kit (SDK) component for application development and debugging. However, during the time period of this thesis, Xilinx released Vivado, a new suite intended to replace ISE; however, due to its lack of support of the FPGA in use [Xilinx'12], this suite has not been used in most of the development. Nevertheless, the necessary considerations to move to this platform and newer FPGA families are detailed in $\$ 5.4$, with an example implementation of a complete video filtering system using this platform described in $\S 7.3$.

All hardware modules-the so-called intellectual property cores (IP cores)— developed in this thesis have been elaborated using VHSIC hardware design language (VHDL). Applications and experimental tests have been written using the $\mathrm{C}$ programming language, which is also employed for the elaboration of computer simulations of the hardware platform for compatibility reasons.

Offline image and data processing has been performed mostly using Python language, and image conversion from and to raw grayscale format has been facilitated by the open-source ImageMagick ${ }^{\circledR}$ image manipulation software suite. 


\subsection{THESIS STRUCTURE AND ORGANIZATION}

This section describes the organization of the present thesis document.

\subsubsection{Structure and hierarchy of the thesis}

This thesis studies, discusses, and develops several topics and ideas related to EHW and DPR. The present document is organized by topics, with each chapter covering a different topic. Each chapter is further divided into sections (\$), each discussing a separate idea or research line of the thesis. Sections are in turn subdivided into subsections, which describe separate parts of the research line.

\subsubsection{Organization of the rest of the thesis}

The rest of this thesis document is organized as follows.

Chapter 2 provides a technical background for this thesis. This chapter starts by describing the concept of EHW, as well as some of the most common EAs, topologies, and reconfiguration methods employed to implement it. After that, the state of the art showing the most relevant recent work in the topic is presented. The chapter concludes by identifying the potential lines of research to explore.

Chapter 3 describes a preliminary implementation of a DPR-based EHW system: a reconfigurable image noise filter based on a systolic array (SA). This work was developed in 2011 as part of the B.Sc./M.Sc. final project of the author [Mora'11], which stems from research lines in the fields of EHW [Salvador'15] and DPR [Otero'14], providing a proof of concept for those research lines. This implementation has been used as the base use case for the thesis, with all the research lines revolving around the improvement and generalization of said use case. The results of this chapter demonstrate the feasibility of the proposed EHW system, showing that it is able to find solutions that outperform other methods that are commonly used for the task in consideration, taking just a few minutes to evolve. Additionally, properties of the system such as generalizability of the solutions, adaptivity to different problems, and fault tolerance are described. The chapter concludes exposing a series of weaknesses of the described system, motivating the analysis and development performed in the following chapters. 
The following chapters discuss different changes in the design methodology oriented towards improving the capabilities of the system and simplifying its utilization and extension to different problems. Chapter 4 focuses on the reconfiguration methodology. In this chapter, a DPR methodology that primarily targets fine grain reconfiguration is presented. This methodology greatly simplifies the reconfiguration process of fine grain reconfigurable architectures like the one at hand, which results in a reduction of the reconfiguration time by a factor of 4 times and facilitates the development of new applications. Additionally, the chapter describes the development of a software framework for the acquisition of partial reconfiguration data to be used in DPR.

Chapter 5 describes the different advances made in the field of the hardware architecture. The main goal sought in this chapter is to improve the processing architecture in terms of operating frequency and resource usage. In effect, a change in the design methodology for the reconfigurable architecture manages to achieve a much higher operating frequency (from $100 \mathrm{MHz}$ to more than $400 \mathrm{MHz}$ ) which further reduces the overall evolution time, as well as a lower resource usage ( 2.5 times smaller than the best achieved in the preliminary work) and a much higher flexibility in the scalability of the design. This has led to the possibility of developing considerably larger processing arrays, from original sizes ranging from $4 \times 4$ to $8 \times 7$ processing

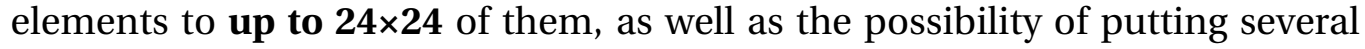
of these arrays in parallel in order to accelerate the evaluation of candidate solutions. This newfound scalability is evaluated in order to assess the benefit of larger arrays in terms of quality of the obtained solutions. Additionally, a comparative study between two different EHW topologies-SA and Cartesian genetic programming - in terms of scalability and resource usage is performed, concluding that the SA topology is the best choice for the use cases in scope. Finally, a change in the operating paradigm is proposed, which enables the system to be used in combination of more modern technologies, thus promoting its integration into future projects.

Chapter 6 discusses several improvements on the EA which aim at reducing the overall evolution time, both by making it more efficient in finding acceptable solutions and by exploiting the parallelization capabilities proposed in the previous chapter in an efficient manner. This results in a reduction of the evolution time by a factor of $\mathbf{3 7}$ times, which, combined with the previous improvements in the reconfiguration and processing speed, results in an overall reduction of the evolution time from several minutes to less than two seconds. This greatly increases the range of applications this system can 
be used in, including those with strict real-time requirements that would have previously been impossible to fulfill.

Chapter 7 changes the context from improving the system to exploring potential applications that may benefit from the current system, and studying how this system would be implemented and used in such cases and which modifications would be necessary to perform.

Finally, chapter 8 concludes the thesis, by comprehensively summarizing its main contributions, analyzing the impact this thesis has had in the state of the art, and establishing some guidelines and suggestions for future research lines. 


\section{TECHNICAL BACKGROUND}

Before proceeding with the research work this thesis has involved, some fundamental concepts of EHW and previous work related to the topic have been analyzed and described. This chapter covers the technical background of this thesis, from the description of concepts related to evolutionary design and evolvable hardware to the state of the art in the subject.

Section 2.1 provides a general description of the concept of EHW.

Afterwards, in section 2.2, the main aspects of EHW are described: evolutionary algorithms employed in their training stage, different interconnection topologies the building blocks of modular adaptive hardware may take, and reconfiguration techniques used to change the functionality of said building blocks during or after the evolution.

Then, section 2.3 presents the state of the art of this thesis, summarizing the most prominent works in the field of evolvable hardware.

Finally, section 2.4 enumerates the different topics of the field in which research may prove profitable.

\subsection{INTRODUCTION TO EVOLVABLE HARDWARE}

Typically, development of a system targeting a problem, be it a software system, a digital hardware application, or any other kind of system, involves deep knowledge of the problem to solve in order to properly design the system. For example, a system in charge of enhancing a picture may be designed using different techniques that have been previously studied and mathematically assessed, such as Fourier analysis, median filtering, FIR/IIR filtering, convolution... 
This traditional approach has two disadvantages. First, it involves knowing precisely what kind of problem needs to be solved, which, in situations where the problem conditions are unpredictable and fluctuate over time, may imply an analysis and classification of the current conditions in order to choose the most suitable solution. Second, it requires developing a system that will target that specific problem or problems, which in the case of varying conditions may involve making the system able to implement a set of different solutions, for example via adjustable parameters or selectable functional modules that will be configured according to a predetermined specification.

Adaptive systems, on the other hand, follow a different approach: instead of having a fixed functionality targeting a specific problem, they have a flexible parameterized functionality that is configured using an optimization algorithm that will find the solution that best suits the current problem conditions. Typically, adaptive systems have a set of modifiable parameters that are adjusted by the optimization algorithm. An example of such a system is an adaptive filter, which is a linear filter (FIR or IIR) whose coefficients can be adjusted [Mathworks].

In order to work, an adaptive system requires a training stage in which it will be adapted to a specific requirement. During this stage, the system will process a training dataset representing the problem that needs to be solved, and compare the result with a reference dataset representing the desired result. For example, an image filtering application used to remove noise from images could use an arbitrary test image as a reference, and the same image with some artificially added noise as a training input. The system will try different configurations, using an evaluation function typically known as fitness function or cost function, which calculates a numerical value indicating the quality of the solution based on the discrepancy between the output and the ideal reference. This fitness value is used in the optimization algorithm as the comparison criterion to determine which solutions are considered better. Seen as an optimization problem, the fitness function is the objective function to optimize (maximize or minimize).

Finally, once the system has been trained for a certain problem, it will—or should - be able to process other datasets that present a similar problem. This is, the solution "learned" by the system for a certain dataset is not limited to that dataset; it can be generalized to other ones with a similar problem. However, it is possible that a certain adaptive system overfits a solution, this is, learns a method to generate specifically the training output. This can typically be avoided by using large enough datasets

Evolvable hardware (EHW) is a realization of an adaptive system in hardware that uses an evolutionary algorithm (EA) to find the optimal 
configuration for a specific problem. Rather than adjusting parameters, EHW is typically configured by changing the functionality and interconnection scheme of small functional blocks known as processing elements (PEs). These PEs may implement functions of varying degrees of complexity, ranging from simple logic gates [Miller'99] to complex processing modules that may behave as microprocessors [Kung'78].

EHW can be evolved extrinsically or intrinsically [Garis'94]. In extrinsic evolution, the different configurations that are tested by the EA are not evaluated in the actual EHW system; instead, their result is computed in a simulated implementation of the system, eventually configuring the EHW with the optimal solution when the EA has finished. Intrinsic evolution, on the other hand, performs these evaluations directly on the EHW system. The latter has the advantage of not needing an absolutely accurate model of the system (which may not be available or be excessively costly from a computing point of view); additionally, it will include the effect of subtle differences from this theoretical model the hardware may be subject to, such as noise sources, timing issues, or degradation of the hardware, which enables the realization of fault-tolerant systems [Thompson'95, Tyrrell'01, Salvador'11b]. Additionally, it would allow making the EHW system completely autonomous, needing only a processor to host the EA (and not the whole simulation), and thus can be very beneficial for systems where dependency on external devices may be infeasible.

Field-programmable gate arrays (FPGAs) provide an excellent platform for the implementation of digital EHW systems, since they allow the realization of arbitrarily complex digital circuits providing a specific functionality with a high performance and low power consumption (compared, for instance, to a CPU or general-purpose GPU) [Nurvitadhi'17]. Furthermore, some FPGAs are able to perform dynamic partial reconfiguration (DPR) [Lie'09], this is, the ability to change the configuration of a fragment of the configured circuit without stopping or modifying the rest of the system. This has been seen as an enormously beneficial feature for the development of EHW systems, which could implement both evolvable and static parts on a single system and perform intrinsic evolution.

EHW has a wide range of possible applications, which can be motivated by multiple reasons: the need to generate solutions for problems that are not known in detail, the need to adapt to varying working conditions (such as different problems or performance requirements), and the ability to overcome potential faults in the system due to degradation of the hardware or exposition to hostile environments [Yao'99]. One example of such a situation would be a satellite application [Veljković'13]: satellites operate in an environment subject to high amounts of radiation that may interfere and degrade the 
equipment, thus benefiting from the fault tolerance provided by intrinsic EHW; additionally, communication with satellites has a high latency, so the on-board application should be able to autonomously adapt to different tasks without dependency on external commands.

\subsection{IMPLEMENTATION TECHNIQUES FOR EVOLVABLE HARDWARE SYSTEMS}

In the previous section, the concept of EHW has been described. This section provides details on the implementation techniques for the main aspects of EHW.

First, the concept of evolutionary algorithm is described, and some terms related to EAs are defined. Additionally, the main types of EA are described. Then, a description of the most common PE interconnection topologies used in EHW and other PE-based systems is provided, highlighting the most prominent features of each of them, and pointing out the advantages and disadvantages each approach would present in the context of DPR-based EHW implementations. The mission of the EA will be to define the behavior and interconnection options of those PEs. Finally, a series of different mechanisms that can be used for changing the functionality of the PEs as directed by the EA is described.

The aim of this section is to provide the reader with a background of the existing techniques for a better understanding of the challenges this thesis and the works in the state of the art have confronted.

\subsubsection{Evolutionary algorithms}

An evolutionary algorithm (EA) is a stochastic optimization algorithm, this is, a method to find optimal solutions to a problem based on a random search rather than a systematic search. The word "evolutionary" comes from the fact that these algorithms are inspired by Darwin's theory of evolution by natural selection: rather than following an absolutely random search of candidate solutions, EAs follow a progressive search in which new solutions 
are explored by combining and modifying previously tested solutions, favoring those solutions which present a more desirable behavior and discarding those that don't.

In order to compare different solutions, EAs rely on a figure of merit of each solution known as fitness. This will be a representative value of how desirable a solution is, so that two solutions can be compared by comparing their fitness values. The fitness function is a mechanism used to calculate the fitness of a solution, and typically consists in evaluating the solution against a specific known problem. For example, an evolvable noise filter could have a fitness function that consists in comparing the output of the filter under a known noisy input data set with the ideal noise-free data set, calculating the fitness as the error value between these two, for example as a root mean square error (RMS) or as the peak signal-to-noise ratio (PSNR). Therefore, one solution will be considered better than another if its RMS error is lower or its PSNR is higher, for instance.

The set of all possible solutions that can be explored by the search algorithm is known as search space. The no free lunch theorem [Wolpert'97] postulates that no search algorithm is better than a random search for every arbitrary problem; an algorithm that works better in some cases will work worse in others. However, some search algorithms can be determined to be more efficient than others for certain types of problem. This efficiency will depend on the ruggedness of the fitness landscape, which is an abstract representation of the fitness values of all elements in the search space as different heights on a terrain (Figure 2-1). Hence, a hill-climbing algorithm might be a good solution if the fitness landscape is smooth (with a single or few local optima, as shown in Figure 2-1, left), but if the fitness landscape is rugged (containing several local optima) this algorithm would likely get stuck at a local optimum and would be unable to find better solutions: a more suited search algorithm would be one able to randomly "jump" to different points in order to avoid getting stuck at these local optima (Figure 2-1, center), as is the case for EAs. EAs rely on the assumption that the fitness landscape will be relatively smooth, and thus small changes in a solution will generally result in small changes in its fitness (unlike the one shown in Figure 2-1, right); nevertheless, they are designed to tolerate a certain amount of ruggedness.

Normally, the search algorithm does not rely on directly modifying the solution, but instead uses an abstract representation of that solution, which is typically described as a sequence of bits, numbers, or symbols. This representation is known as genotype or chromosome of a solution, with each element in the sequence known as a gene.

All EAs follow a similar pattern: 

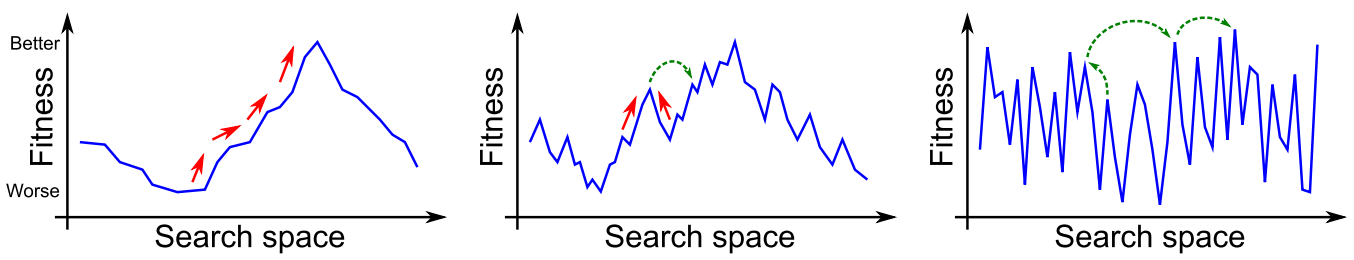

Figure 2-1: Fitness landscape examples. Left: smooth landscape with a single maximum. Center: rugged landscape with multiple local maxima. Right: extremely rugged landscape; small changes in the solution result in high fitness variations.

- First, an initial population of a certain number of candidate solutions is chosen, either randomly or by using a predefined initial solution.

- Then, an iterative process starts in which the population undergoes the following operations:

- Selection of the best individuals in the population based on their fitness, which will be the parents for the next generation.

- Crossover or recombination, where a new child is obtained by combining parts of the genotype of two or more parents (Figure 2-2).

- Mutation of a genotype, in which some randomly selected genes of the genotype are modified (Figure 2-3), either by flipping them (in the case of binary genes), adding a random value to them, or replacing them by a random value.

- Finally, the evolution is terminated after either a certain number of iterations (or generations) have elapsed, a target fitness value has been reached, or another termination criterion is reached. The best individual in the resulting population will be considered as the result of the evolution.

The specific mechanisms for selection, crossover, and mutation depend on the implementation. For example, selection may consist in picking the best $\mu$ individuals and discarding the rest (elitist selection), picking two individuals at random and choosing the one with best fitness (tournament selection), or using a uniform random distribution proportional to the fitness of the individuals (roulette-wheel selection). Crossover and mutation mechanisms may be more or less complex, and in some cases one of the two may not be used at all. 

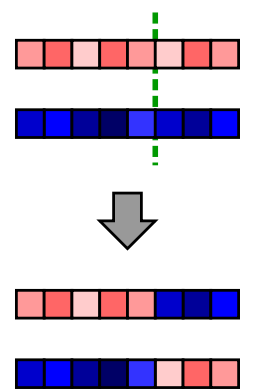
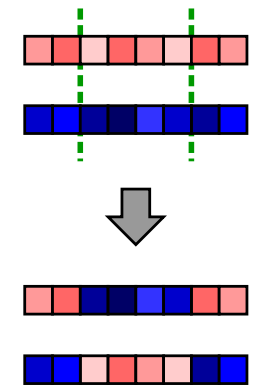
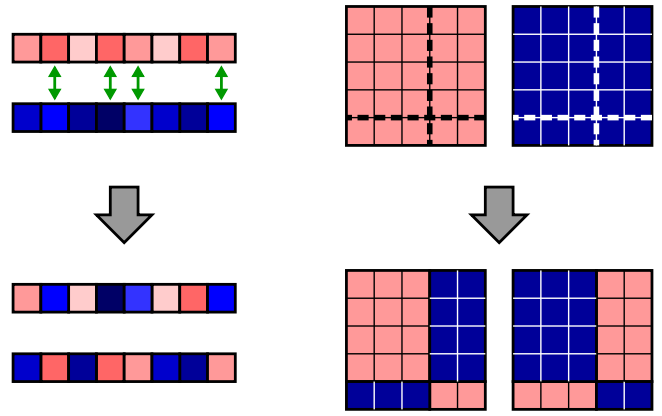

Figure 2-2: Some examples of crossover. From left to right: 1-point crossover, 2point crossover, uniform crossover, and a particular case of crossover designed for twodimensional chromosomes [Moon'01].

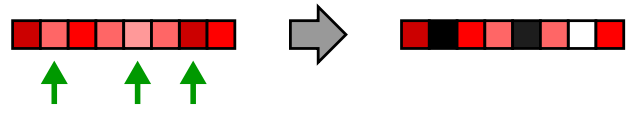

Figure 2-3: Mutation

\section{Main variants of evolutionary algorithms}

Depending on the problem description, genotype representation, and evolutionary operators (crossover, mutation, and selection), a wide variety of EAs can be devised. The most common types of EA are briefly described below. For an exhaustive description and formal definition of these EA variants, the reader is referred to [Bäck'96].

One of the first realizations of an EA was the evolution strategy (ES) [Rechenberg'65, Schwefel'93], developed in the Technical University of Berlin in the 1960s. In this EA, the genotype consists of a sequence of real-valued genes, each of them describing a different parameter of the system to optimize. Mutation consists in adding a normally distributed random value to all genes in the genotype (this random value might be identically distributed for all genes or have an ellipsoidal shape on $\mathbb{R}^{n}$ ). A distinctive feature of this EA is the fact that the standard deviation of this normal distribution may be included as part of the genotype, so that this EA not only evolves the individuals but also the degree in which they may change during the evolution. Originally, this EA did not use crossover, relying exclusively on mutation and selection; this was denoted in [Schwefel'77] as $(1+\lambda)$-ES, indicating that each generation has 1 parent and $\lambda$ children, and that selection is performed on both parent and children. Nevertheless, implementations exist that perform crossover by averaging two individuals; these are known as $(\mu+\lambda)$-ES or $(\mu, \lambda)$-ES (with $\mu$ 
being the number of parents) depending on whether the parents participate in the selection or are systematically discarded.

A similar EA variant is evolutionary programming (EP) [Fogel'62, Fogel'96], which was developed independently from ES in the 1960s. The mutation mechanism in EP consists in adding a normally distributed random value to each gene, but, unlike in ES, the standard deviation of this value is made proportional to the fitness of a solution, so that solutions with a lower (better) fitness will be subject to smaller mutations than those with a higher (worse) one. Crossover is not used at all. This EA variant uses a $(\mu+\mu)$ strategy in which a population of $2 \mu$ individuals is reduced to $\mu$ parents by performing a tournament selection: each new parent is obtained by randomly choosing two or more individuals of the population and picking the best of them.

A more flexible variant of EA is the genetic algorithm (GA) [Holland'75, Goldberg'89], which has become the most widely used form of EA due to its versatility and relative independence with the problem to optimize. Unlike ES and EP, the genotype in GA is typically represented as a sequence of bits, ${ }^{1}$ with each feature of the system being encoded in a specific group of bits. For example, a group of 4 bits at a specific position of the genotype may encode which of 16 possible arithmetic functions is performed by a certain PE. This encoding provides more flexibility than ES, since it can not only represent real-valued system parameters, but also the choice of a specific option within a range (e.g., indexed functions or modules, multiplexer settings, presence or absence of specific features...). Mutation in GA usually consists in randomly selecting a certain number of bits and flipping their value. This more closely approximates biological mutation, which affects a single base pair rather than the whole gene describing a single feature (with each gene typically spanning several thousands of base pairs). In addition to mutation, most implementations of GA heavily rely on crossover, unlike ES, which rarely uses it, or EP, which does not use it at all. The flexibility of this type of EA has made it a very popular choice for most evolvable systems.

Finally, an EA variant that is often used in the field of evolutionary computation is genetic programming (GP) [Koza'92]. GP is specifically oriented to the design of software functions. As such, the genotype is represented as a tree structure, with each node in the tree representing a basic function (such as addition, subtraction, multiplication...). This type of structure allows the representation of arbitrary expressions as used in mathematical or computing contexts; as an example, Figure 2-4 shows

\footnotetext{
${ }^{1}$ Nevertheless, other genotype representations (such as a sequence of integers, each representing one feature) are possible.
} 
the algebraic expression $\frac{-b+\sqrt{b^{2}-4 \cdot a \cdot c}}{2 \cdot a}$ represented as a tree. The crossover mechanism used by GP is very particular to this EA variant: it consists in swapping subtrees between two trees, as shown in Figure 2-5. This results in genotypes potentially growing to arbitrarily large sizes, which would make some solutions impossible to implement in systems with limited resources, so care must be taken to limit the length of the tree (for example, including it as a factor for the fitness computation).

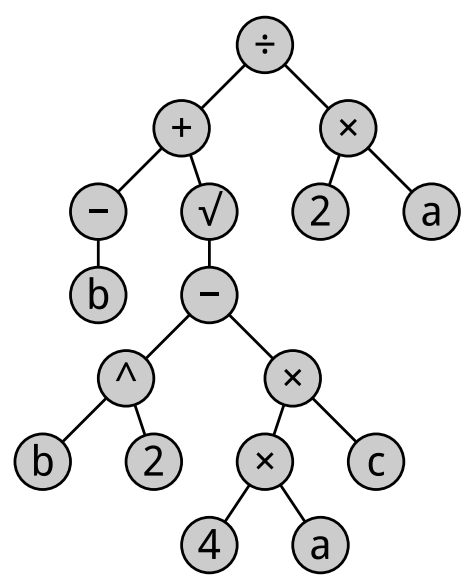

Figure 2-4: Tree representation of the expression $\frac{-b+\sqrt{b^{2}-4 \cdot a \cdot c}}{2 \cdot a}$
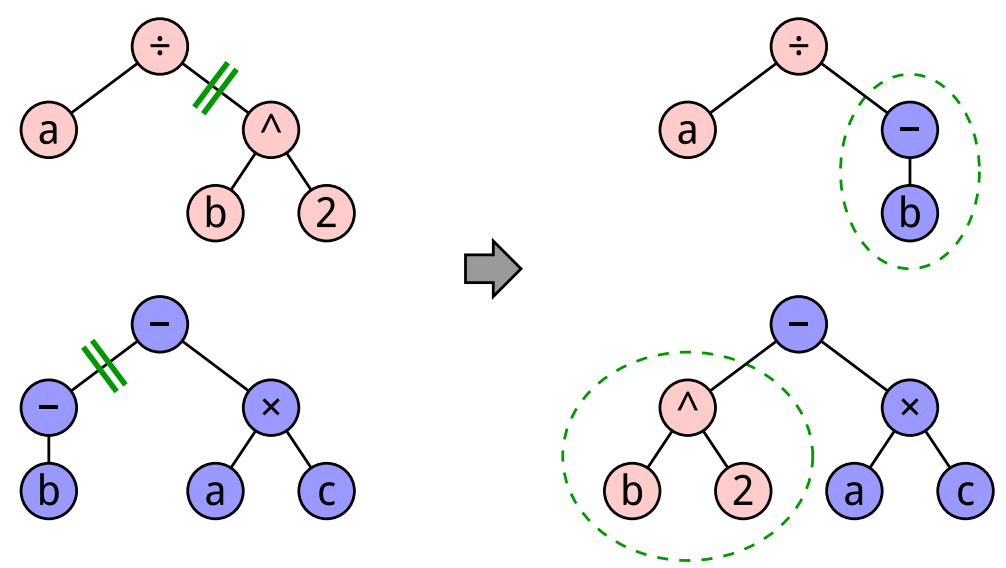

Figure 2-5: Crossover mechanism used in GP

Among all these EA variants, probably the one that is best suited for EHW is GA, since it allows mapping each gene to a specific feature of a basic hardware structure (which function is implemented by each PE, which value does each parameter take, etc), without the implications of a more abstract representation 
such as that of GP. Nevertheless, combinations of the characteristics of several EA variants are also possible, such as the use of GA-like genotype and mutation with the evolution methodology used by EP (tournament selection and no crossover),

\subsubsection{PE interconnection topologies}

As it was said in $\$ 2.1$, EHW systems are typically composed of multiple PEs, each of which performs a specific operation. Depending on the way these PEs are interconnected, different topologies can be obtained. The most common types of PE-based data processing structures-intended for evolvable/adaptive applications or not-are described below, comparing the interconnection topologies used in each of them and analyzing the suitability of these topologies for EHW applications.

The most straightforward way to generate arbitrary digital circuits is inspired in the internal structure of early FPGAs, which consisted of a twodimensional grid of configurable logic blocks (CLBs), with each CLB connected to its neighbors. Thus, early implementations of EHW [Thompson'97] exploited this structure to implement evolvable circuits directly on the FPGA fabric by following a north-east-west-south (NEWS) two-dimensional grid interconnection scheme. As it is shown in Figure 2-6, PEs in this scheme (usually referred to as cells) have an input and an output in each of the four sides, compute a function using some of the inputs, and generate each output as either a copy of an input or the result of the computed function. The outputs of the cells may be unregistered (as is the case for [Thompson'97]), leading to an asynchronous circuit design which may contain feedback loops and rely on the intrinsic delay of logic elements; or registered using a global clock signal (as is the case in [Upegui'06]) so that each cell operates with the data from neighboring cells from a clock cycle earlier. Implementations using the latter scheme are often referred to as cellular automata (CA).

This EHW scheme is very simple and leads to a high degree of flexibility in the range of possible circuit configurations. However, this flexibility may be a disadvantage for EHW since it increases the search space, hindering the effectiveness of the EA. Thus, modern EHW implementations rarely use this approach, and instead use a constrained range of higher-level PE functions and/or a specific interconnection structure.

A considerably widespread computing model used in adaptive systems (specially in the field of artificial intelligence) is the artificial neural network (ANN) [Wasserman'89], which is inspired on the behavior of neurons. An 


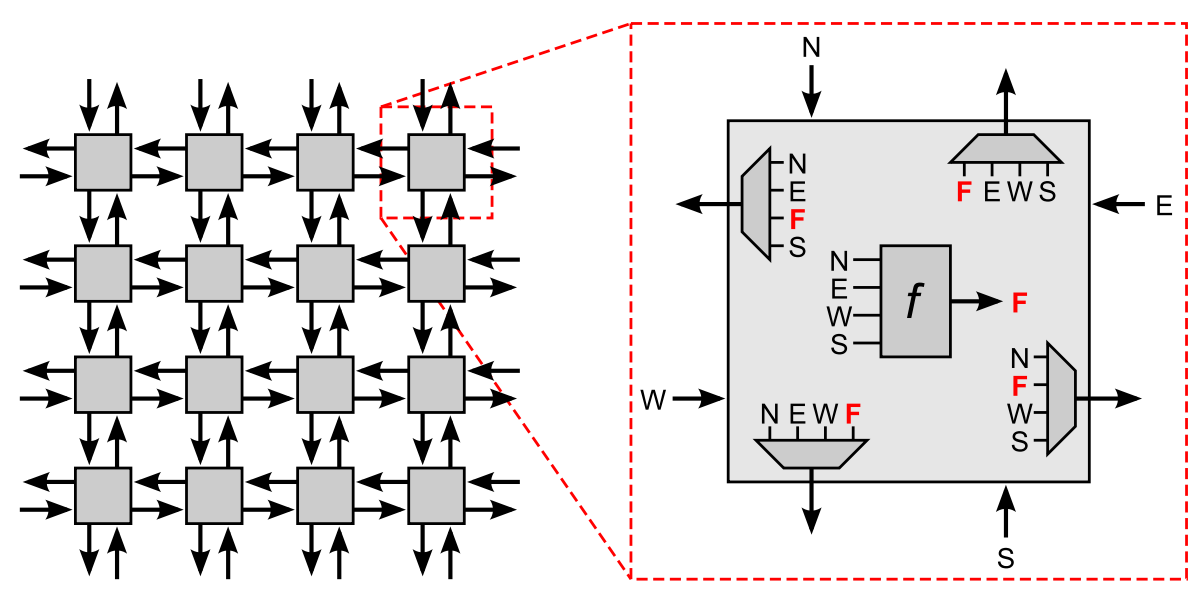

Figure 2-6: “NEWS" two-dimensional grid topology

ANN consists of a series of PEs (referred to as neurons), with the output of each neuron being calculated as a linear combination of its inputs following a formula like $x_{j}=f\left(b_{j}+\sum w_{i j} \cdot x_{i}\right)$, where $x_{i}$ are the different neuron inputs (either outputs of other neurons or primary inputs of the ANN), $w_{i j}$ and $b_{j}$ are the weights and bias used in this calculation, and $f$ is a nonlinear saturation function (e.g., a hyperbolic tangent) known as activation function. An optimization algorithm is used to determine the value of $w_{i j}$ and $b_{j}$. Given the mathematical nature of the problem, ES or EP can be used to optimize these parameters, although other EAs such as GA can be used as well.

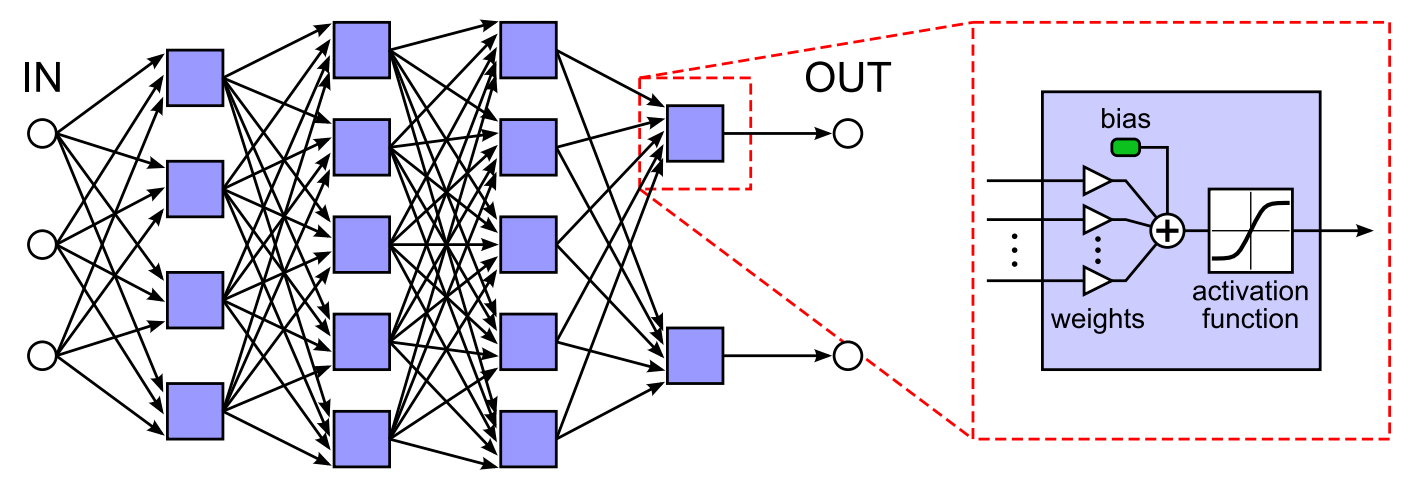

Figure 2-7: Artificial neural network (left) and detail of a neuron (right).

The simplest topology used in ANNs is the multilayer perceptron (MLP) [Rosenblatt'62]. In this topology, neurons are organized in layers, with the neurons of each layer receiving their input from the neurons of the previous layer (as it was the case in Figure 2-7). One benefit of this layered 
interconnection structure from the point of view of hardware design is that it can be easily pipelined by registering the output of each neuron in order to operate at higher clock frequencies; thus, one data vector could be fed in each clock cycle and would propagate forward one layer per clock cycle, without data input at different clock cycles getting mixed: the output will be strictly a function of the input a certain number of clock cycles before. This way, although this structure would have a latency of several clock cycles, its throughput would be one data vector per clock cycle regardless of the depth of the ANN. However, ANNs present a disadvantage: the arithmetic functions they implement consume a considerable amount of resources, so implementing all these operations in parallel would require one DSP unit per each multiplication performed (typically in the order of several millions). ${ }^{2}$ Therefore, if resources are scarce, purely parallel implementations of large ANNs are not possible, requiring the arithmetic operations to be sequenced at the expense of data throughput (or, alternatively, replacing these costly multiplications with an approximate method such as a combination of additions and bit shifts). Additionally, the high number of parameters to adjust (83 in the case of Figure 2-7, but potentially growing to several millions on larger ANNs) makes the search space considerably large, which may result in a poor performance of the EA. Finally, an important issue of FPGA implementations of this interconnection topology is the high complexity of the routing of interconnections between neurons: this complexity may result in high delays on the routes which would result in a poor performance in terms of maximum operating frequency.

An interesting variation of ANN topology that was devised to overcome this last issue of MLP is the block-based neural network (BBNN) [Moon'01]. As shown in Figure 2-8, BBNNs solve the problem of long routes by limiting the connectivity of each neuron to the neighboring ones, which simplifies the routing and makes the topology easily scalable in both dimensions. As it can be seen, neuron-to-neuron connections can go in either direction, so the EA will not only need to generate the weights and biases (up to 6 per neuron, depending on the number of inputs and outputs), but also the direction of each connection. Since direction is a binary parameter and thus cannot be evolved using ES or EP, this topology typically uses a GA to evolve, with a specialized crossover mechanism over a two-dimensional genotype [Moon'01].

Unlike the MLP topology described before, BBNNs may be subject to feedback, since data can flow backwards. This may have the effect of forming

\footnotetext{
${ }^{2}$ As an example, the simple 16-neuron ANN depicted in Figure 2-7 would require 67 multipliers; typical ANN implementations have thousands to millions of neurons. As a reference, the Virtex-5 LX110T FPGA that has been used in this thesis only has 64 DSP slices.
} 


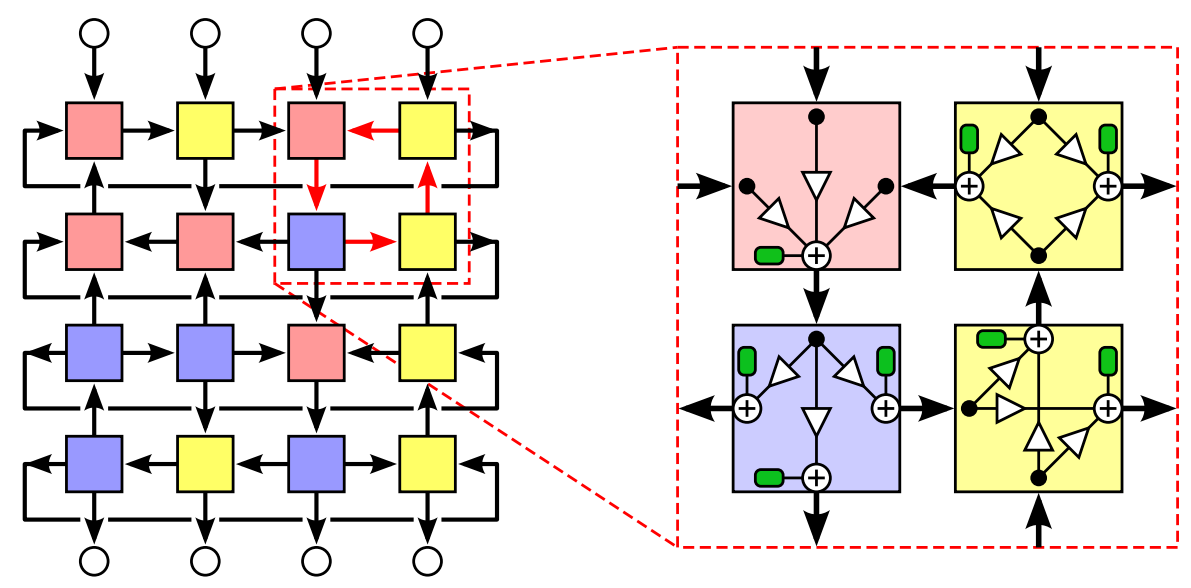

Figure 2-8: Block-based neural network (left) and possible neuron direction modes (right), displaying the cases of neurons with one (red), two (yellow), and three outputs (blue).

closed data loops (as is the case on the four neurons on the top right corner in Figure 2-8), which might result in the network behaving as an infinite impulse response (IIR) system, i.e., the effect of a certain input would remain in the filter indefinitely. Depending on the use case, this may be beneficial because it allows for a more complex functionality with many fewer neurons; on the other hand, it makes the system response to a certain input unpredictable since it depends on the internal state, which might be undesirable. This is avoided in [Merchant'10] by restricting the direction of vertical connections so that they always go downwards. Even with this solution, the overall latency of this network (i.e., the number of clock cycles a certain input takes to affect the output) is not well defined, since data can follow paths of different length, resulting in data from different clock cycles getting mixed; whether or not this is an issue depends on the particular problem. ${ }^{3}$

Several other ANN topologies exist; for example, [Garis'02] proposes a three-dimensional BBNN-like topology.

A very popular topology that was devised specifically for EHW is Cartesian genetic programming (CGP) [Miller'99]. Unlike ANNs, CGP focuses on digital circuit generation rather than a mathematical neuron model. As shown in Figure 2-9, the CGP topology consists of a series of PEs arranged in a two-

\footnotetext{
${ }^{3}$ The effect of this on a system where data is fed at a rate of one vector per clock cycle is that the output is a function of multiple time-shifted inputs. In $§ 5.3 .3$ it will be discussed how having this type of "fuzzy" latency can negatively impact the performance of the system at hand. [Merchant'10] overcomes this by establishing a token-based synchronization scheme, at the cost of a lower data throughput.
} 
dimensional grid (hence the name "Cartesian") in a similar way to MLPs, but, unlike those, each PE has a limited number of inputs (typically two), and implements a specific operation from a list of possibilities on those inputs (usually simple functions such as additions or logic operations). These inputs may be connected to a primary input or to the output of a PE from one of the columns to their left. An EA is in charge of deciding which operation is performed by each PE and which primary inputs or PEs are connected to its input (often restricting the number of columns to the left a source PE may be, in order to limit the size of the search space). This topology receives its name from the fact that it is able to implement tree-like functions such as those used in GP; however, implementations typically use variations of GA for its evolution (usually without crossover) rather than GP.

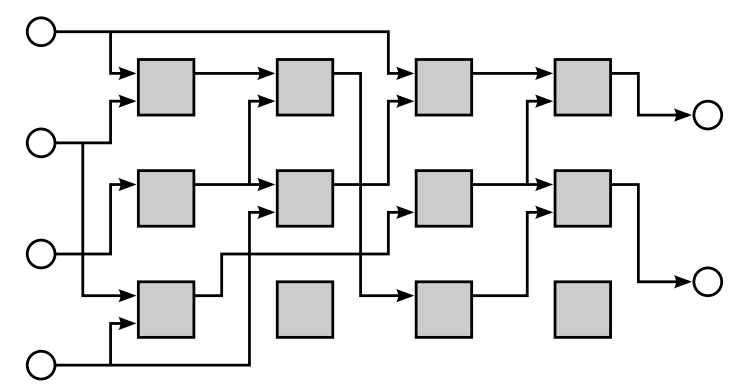

Figure 2-9: Cartesian genetic programming topology

Same as with MLP, this topology is easy to pipeline so that the output is kept strictly as a function of the input with a fixed latency. Additionally, the use of simple functions chosen from a list instead of weighted average functions considerably reduces the resource usage for each PE (as well as the search space, since each PE is encoded as a single function index and twoor more-input indexes, rather than as a long list of real values). However, FPGA implementations of CGP have the same routing issues as MLP, which might limit their maximum operating frequency. Additionally, although the logic resources needed to implement the PE operations have been substantially reduced, the fact that each $\mathrm{PE}$ input can be received from a wide range of sources implies using large multiplexers, which might use more resources than the PE itself. ${ }^{4}$

Finally, an interconnection topology that has been used for EHW implementations is the systolic array (SA) [Kung'78]. This topology consists of a series of interconnected PEs, with multiple interconnection schemes existing;

\footnotetext{
${ }^{4}$ The implications of the use of these multiplexers in both resource usage and computational capabilities will be discussed in $\$ 5.2$.
} 
some examples of such schemes are shown in Figure 2-10. The name "systolic" refers to the fact that the output of each PE is registered, leading to a pipelined topology where data moves in wavefronts through the array in "heartbeats" of one clock cycle; thus, each PE is able to process one input vector per clock cycle. It should be noted that SA was not originally meant as an EHW topology; its application to EHW was originally proposed in [Salvador'11a].
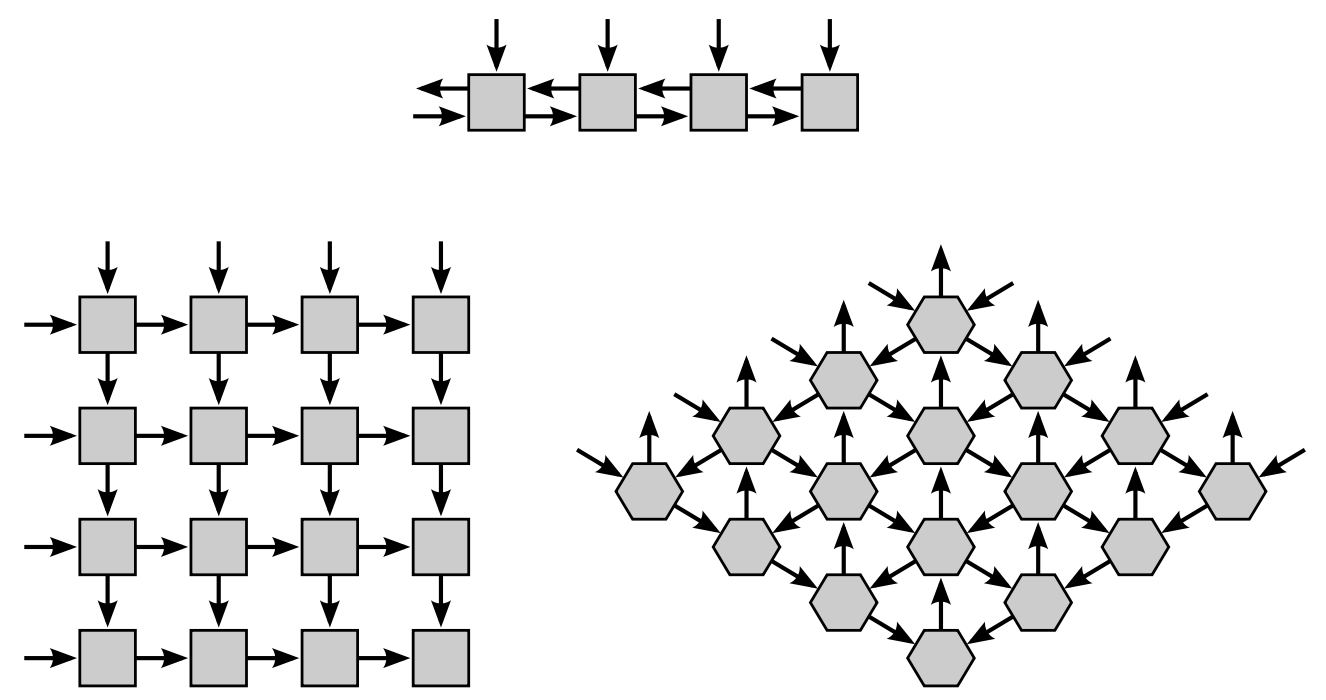

Figure 2-10: Three examples of systolic array topologies: linear (top), orthogonal (left), and hexagonal (right).

The motivation for using SA as an EHW topology is that it solves the routing issues of CGP the same way BBNN does: by limiting the interconnections of each PE to neighboring ones. Unlike in BBNN, these connections are unidirectional. This limitation on the interconnection possibilities reduces their length (thus simplifying the routing and increasing the clock performance) and simplifies the search space, since each PE is defined solely by the operation it performs and not the configuration of its inputs. The pipelining characteristics of SA are discussed in detail and exploited in [Kung'84]: it is shown that, with the correct sequencing of inputs, it is possible to obtain a strict functional dependency of output from input (as with MLP and CGP).

From all these topologies, the one that is best suited for DPR-based EHW is SA, with the functionality of each $\mathrm{PE}$ (both operation and order of its inputs) being changed by partially reconfiguring it. Since each PE is only connected to its neighbors, no additional space is needed for routing the interconnections outside the reconfigurable regions, leading to a compact design that maximizes the resource availability for PEs in a certain area. 
Additionally, the SA topology is easily scalable to arbitrary sizes without changing the PE structure. CGP, on the other hand, would require using multiplexers in addition to DPR, or a much more sophisticated reconfiguration methodology for the interconnections (e.g., an interconnection matrix), and would not be as easily scalable. ANN-based topologies are generally not well suited for high-throughput DPR-based FPGA implementations of EHW for the previously mentioned problems: aside from having an excessive resource usage (which can be solved by replacing the averaging functions with simpler ones), MLPs require a complex interconnection routing that may result in a low value for the maximum operating frequency, and grid-based ANN topologies such as BBNN are affected by a latency uncertainty that results in data from different clock cycles being mixed or some form of data arbitration being needed.

All these reasons have motivated the choice of SA as the PE interconnection topology for the implementation of DPR-based EHW in the research line under which this thesis has been developed. Throughout this thesis, suitability of DPR-based SA implementations as a reduced-size, compact, and high-speed solution for EHW will be proved.

\subsubsection{Reconfiguration methods}

Once EA and hardware architectures used in EHW have been described, the remaining question is how to reconfigure the elements of the EHW circuit, this is, how to modify the functionality of the PEs so that they operate as mandated by the EA.

The most obvious way to generate different circuit configurations for an FPGA implementation of EHW is to synthesize them separately on a computer and loading the obtained circuit on the target FPGA. This approach is known as offline evolution. In the early days of FPGA technology, the structure of FPGAs was quite simple and well documented, so it was easy to generate FPGA configurations directly using an EA. One notable example of this approach is the work of [Thompson'97], where random FPGA configurations are generated directly from software running on a desktop PC. However, more modern FPGAs have acquired a high degree of complexity, requiring specialized FPGA circuit synthesis tools (typically vendor-provided and closed source) running on a PC.

Given the latency involved in synthesizing a solution (which may take from several minutes to several hours) and uploading it from the computer to the FPGA, this solution is generally only feasible as the last step in an extrinsic evolution process, where the EA has been run on a simulated model and the eventually obtained solution has been synthesized in an automated manner. 
Therefore, this approach is not suited for intrinsic EHW systems: these systems must be developed in such a way that their functionality can be modified at run time. This is typically achieved by constructing an overlay on the FPGA, i.e., a basic "skeleton" of a generic circuit, the parts of which can be individually configured at run time using different methods.

When the configuration of EHW can be expressed as a mere series of parameters (as is the case of ANN), the reconfiguration method may consist simply in writing the desired values on specific registers. This idea can be extended to more heterogeneous systems, such as those in which a PE can perform one among a list of operations (e.g., CGP or SA). This led to the creation of virtual reconfigurable circuits (VRCs) [Sekanina'03]. In a VRC, each PE contains all of the functions that it can implement; the output of the PE will be selected through a multiplexer (Figure 2-11). Thus, configuration of a PE is done by writing the function index on a dedicated register. This solution has the advantage of being portable to any platform (FPGA-based or not), and of not having any limitations in the functions a PE can implement, which can be described in HDL. The drawback of VRC is that it is extremely resourceconsuming, since each PE requires implementing all the possible functions simultaneously.

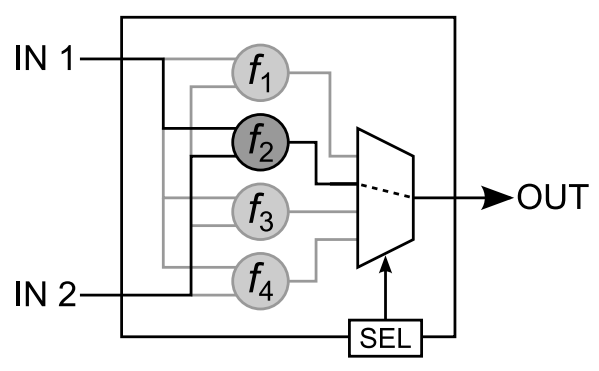

Figure 2-11: Reconfiguration method used by virtual reconfigurable circuits

Since FPGAs can be reconfigured in order to change the circuit they implement, a more resource-efficient solution than VRC would be to replace each PE in its entirety with another one with a different functionality, as depicted in Figure 2-12. This can be achieved thanks to the aforementioned dynamic partial reconfiguration (DPR) capabilities provided by some FPGAs. Specifically, some FPGAs provide a specific port that allows performing this DPR from the FPGA itself, as is the case of the internal configuration access port (ICAP) present in some Xilinx® FPGAs. This approach provides the same versatility in the possible functionalities that can be implemented as VRC with a much lower resource usage, but has several drawbacks: first, it is not as portable as VRC, requiring specific FPGAs and a different design flow 
for each FPGA family; second, it requires special care to be taken so that the interconnections crossing the boundaries of reconfigurable regions are kept consistent (which is solved when using dedicated partial reconfiguration dataflows that the synthesis tool needs to provide, or with techniques such as the use of bus macros in order to fix these interconnections [Lim'04]); and third, the reconfiguration process may be relatively time-consuming, resulting in the evolution process being slowed down. Therefore, in many cases it would be preferable to use VRC for the implementation of EHW systems rather than DPR; nevertheless, the ability to implement a much larger number of PEs when the resources are limited is an attractive feature that has drawn the attention of the research community, making DPR-based systems a promising research line.

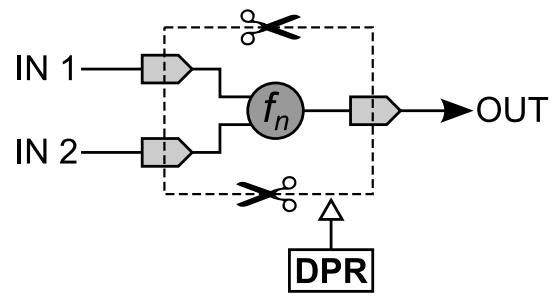

Figure 2-12: Reconfiguration method used in dynamic partial reconfiguration

In order to overcome the difficulties DPR involves (specially those related to the routing of interconnections), an approach that is used in some FPGA implementations of EHW is to only modify the logic component of a circuit, leaving the interconnections intact. FPGAs implement most of their basic digital functions (both logic and simple arithmetic such as addition) by using binary look-up tables (LUTs). A LUT is a digital component presenting an input of several bits (the address) and an output of one or more bits; the output of a LUT is obtained by looking up the output value corresponding to a certain input in a memory (Figure 2-13). Thus, a LUT with enough inputs and outputs is able to perform any logic operation, provided that the right binary pattern is stored in its memory. Hence, LUTs are often used in FPGAs as the building blocks for logic functions. LUT-based reconfiguration of a circuit consists in changing its behavior by modifying the content of these LUTs and thus the function they implement. This can be achieved in two ways: by performing DPR of those LUTs while leaving the interconnections intact, or by using the shift-register LUT (SRL) functionality available in some LUTs of certain FPGAs, where these LUTs double as shift registers and thus their content can be rewritten by shifting in a new configuration.

Although LUT-based configuration solves the complexity of handling the interconnections and reduces the configuration time, it restricts the range of 


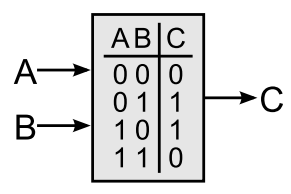

Figure 2-13: A simple 2-input look-up table implementing the XOR function

functions a LUT-based PE can implement, since all functions will need to be implemented following an identical structure of logic operations. Additionally, SRLs have the disadvantage of being less abundant (specifically, in Xilinx Virtex-5 and 7 Series FPGAs, only $25-30 \%$ of LUTs have SRL functionality; additionally, while LUTs in these FPGAs have 6 inputs, SRLs only use 5 of these inputs, and thus cannot implement the same range of logic functions). LUTbased DPR overcomes this last issue since every LUT in the FPGA is eligible for being reconfigured, but this is at the cost of being more technology-dependent, due to the use of DPR.

Among all the described reconfiguration methods, probably the most common is VRC, since it removes the technological barriers and restrictions imposed by DPR and LUT-based reconfiguration. However, as it has been said, it is considerably inefficient in resource usage. DPR and LUT-based solutions, on the other hand, allow a much more efficient use of the resources, thus allowing to implement much more complex solutions in small FPGAs, so research in this field is promising. DPR-based EHW implementations provide a much higher flexibility in the range of possible PE functions than LUT-based reconfiguration, at the cost of a more complex and technology-locked design flow. However, since EHW is based in the approximation of functions by using simple functions, this flexibility might not be necessary at all, and PEs could implement simple or even approximate functions and still lead to satisfactory solutions. 


\subsection{STATE OF THE ART}

The most notable works on evolvable hardware in the state of the art are listed in Table 2-1. The columns in this table show the bibliographic reference, a description of the use case application, FPGA family or alternative platform used, reconfiguration method ("Rcfg."), PE interconnection topology ("Topol."), number of PEs, and the type of search algorithm used (evolutionary or not).

It can be seen that early implementations of EHW relied on offline generation of random circuits. Then, as interest on autonomous systems grew, VRC-based implementations gained popularity. Afterwards, the emergence of self-reconfigurable FPGAs enabled the development of more resource-efficient EHW implementations using DPR; however, this comes at the cost of a large reconfiguration time overhead and a very constrained design process. For this reason, several modern implementations of EHW rely on DPR of LUTs, which reduces this time without affecting the PE functionality for simple PE specifications.

Table 2-1: State of the Art

\begin{tabular}{|c|c|c|c|c|c|c|}
\hline Reference & Application & FPGA & Rcfg. & Topol. & PEs & EA \\
\hline [Higuchi'93] & $\begin{array}{l}\text { Evolution of a } 6 \text {-multi- } \\
\text { plexer }\end{array}$ & GAL16Z8 & offline & $\begin{array}{l}\text { GAL } \\
\text { fabric }\end{array}$ & 8 & GA \\
\hline [Thompson'97] & Tone discrimination & XC6200 & offline & NEWS & 100 & GA \\
\hline [Higuchi'96] & $\begin{array}{l}\text { Pattern recognition / } \\
\text { robot control }\end{array}$ & $\begin{array}{l}2 \times \mathrm{XC} 4000 \\
/ \mathrm{ASIC}\end{array}$ & DPR-VRC* & $\begin{array}{l}\text { CGP- } \\
\text { like* }\end{array}$ & 140 & GA \\
\hline [Miller'99] & Boolean functions & - & - & CGP & $100-256$ & GA/PH* \\
\hline [Tørresen’00] & $\begin{array}{l}\text { Road image recogni- } \\
\text { tion }\end{array}$ & XC6200 & offline & CGP & 128 & GA \\
\hline [Moon'01] & $\begin{array}{l}\text { Pattern classification / } \\
\text { robot control }\end{array}$ & (any) & DPR & BBNN & 5 & GA \\
\hline [Garis'02] & $\begin{array}{l}\text { Real-time robot con- } \\
\text { trol }\end{array}$ & $72 \times \mathrm{XC} 6200$ & VRC & $\begin{array}{l}3 \mathrm{D} \\
\text { BBNN }\end{array}$ & 1000 & GA \\
\hline [Sekanina'03] & $\begin{array}{l}\text { Image operators and } \\
\text { filters }\end{array}$ & Virtex/II/E & VRC & CGP & 29 & $\mathrm{GA}^{*}$ \\
\hline [Upegui’06] & $\begin{array}{l}\text { Random Boolean net- } \\
\text { work }\end{array}$ & Virtex-II & LUT-DPR & NEWS & 30 & $\mathrm{CP}^{*}$ \\
\hline [Vašíček'07b] & Image filtering & Virtex-II Pro & VRC & CGP & 32 & $\begin{array}{l}\mathrm{RS}^{*} / \mathrm{HC}^{*} \\
/ \mathrm{GA}^{*}\end{array}$ \\
\hline
\end{tabular}


Table 2-1: State of the Art (continued)

\begin{tabular}{|c|c|c|c|c|c|c|}
\hline Reference & Application & FPGA & Rcfg. & Topol. & PEs & EA \\
\hline [Glette'07] & Face recognition & Virtex-II Pro & VRC & $\mathrm{CDM}^{*}$ & 48 & GA \\
\hline [Glette'08] & $\begin{array}{l}\text { Prosthetic hand con- } \\
\text { trol }\end{array}$ & $\begin{array}{l}\text { Virtex-II Pro } \\
\text { (or better) }\end{array}$ & VRC & $\begin{array}{l}\text { CGP/ } \\
\text { CDM }^{*}\end{array}$ & $50-250$ & GA \\
\hline [Glette’09] & $\begin{array}{l}\text { Sonar return / face } \\
\text { recognition }\end{array}$ & Virtex-II Pro & SRL & $\mathrm{CDM}^{*}$ & $120(\times 2)$ & GA \\
\hline [Merchant'10] & $\begin{array}{l}\text { Bit parity / image clas- } \\
\text { sification / prediction }\end{array}$ & Virtex-II Pro & VRC & BBNN & $4-40$ & GA \\
\hline [Otero'11b] & Image filtering & Virtex-5 & DPR & SA & 16 & $\mathrm{GA}^{*}$ \\
\hline [Salvador'11a] & Image filtering & Virtex-5 & DPR & SA & 16 & $\mathrm{GA}^{*}$ \\
\hline [Salvador'11b] & $\begin{array}{l}\text { Fault-tolerant image } \\
\text { filter }\end{array}$ & Virtex-5 & DPR & SA & 16 & $\mathrm{GA}^{*}$ \\
\hline [Gallego'13c] & Scalable image filter & Virtex-5 & DPR & SA & 56 & $\mathrm{GA}^{*}$ \\
\hline [Mora'13b] & $\begin{array}{l}\text { Noise-agnostic image } \\
\text { filtering }\end{array}$ & Virtex-5 & DPR & SA & 16 & $\mathrm{GA}^{*}$ \\
\hline [Bartolini'13] & (any; not specified) & Virtex-5 & SRL & $\begin{array}{l}\text { CGP- } \\
\text { like** }\end{array}$ & $\begin{array}{l}512 \\
(16 \times 32)\end{array}$ & GA \\
\hline [Dobai'14] & Image filtering & Zynq-7000 & $\begin{array}{l}\text { LUT-DPR } \\
+ \text { VRC }\end{array}$ & CGP & $32(\times 6)$ & $\mathrm{GA}^{*}$ \\
\hline [Glette'14] & $\begin{array}{l}\text { Sonar return / face } \\
\text { recognition }\end{array}$ & Virtex-5 & LUT-DPR & $\mathrm{CDM}^{*}$ & 160 & GA \\
\hline [Dobai'15] & Image filtering & Zynq-7000 & $\begin{array}{l}\text { LUT-DPR } \\
+ \text { VRC }\end{array}$ & CGP & 32 & $\mathrm{GA}^{*}$ \\
\hline [Mora'15] & Image filtering & Virtex-5 & LUT-DPR & SA & $64(\times 8)$ & $\mathrm{GA}^{*}$ \\
\hline [Mora'18b] & Image filtering & Virtex-5 & LUT-DPR & SA & $64(\times 12)$ & GA* \\
\hline
\end{tabular}

GA* Mutation-only GA (no crossover).

CP* Cellular programming (a type of CA-oriented EA).

RS* Random search.

HC* Hill climbing.

PH* Probabilistic hillclimber.

DPR-VRC* Reconfiguration of VRC-like multiplexers using DPR.

CDM* Category detection module (see reference).

CGP-like* CGP with fixed-function PEs (one of each in each column).

CGP-like** Two-level hierarchical CGP-like structure $(4 \times 4$ modules of $4 \times 8$ LUTs) with fixed interconnections. 


\subsection{RESEARCH OPPORTUNITIES}

In view of the state of the art of EHW systems and applications, the following aspects of the field have been considered to be interesting to research.

The main goal of this thesis is to enable the easy adoption of the EHW paradigm for different applications. However, most implementations of EHW are highly application-specific. Thus, an important research path is the exploitation of the integrability and reusability of EHW systems and tools, aiming for a generalized design methodology, which should focus on a more application-agnostic perspective, but with specific real-life applications in mind. The result of this work should be a series of utilities and methodologies that allow the integration of EHW into practical systems.

An important aspect of EHW design that has been taken into account is the resource usage of the system: although FPGAs become larger as newer families are released, so do the requirements of applications. As it has been explained, DPR-based EHW provides a high degree of resource efficiency and flexibility in the design. Therefore, exploiting the capabilities of DPR for the development of resource-efficient EHW may be a profitable research line. Specifically, the SA topology has been proven to be advantageous for the implementation of EHW; however, little work has been done in the field, making the research of the capabilities of this topology and its possible variations an important line of work. In particular, it would be interesting to make a comparison between SA and CGP in terms of resource usage and computational performance, in order to determine which topology is better suited for EHW.

A disadvantage of DPR-based EHW is the difficulty of design: DPR introduces a series of obstacles that must be overcome, due to the strict requirements of placement and routing of interconnections, which impose an additional level of constraints. Thus, research on the automation of the design methodology and development of tools to aid in this design become necessary for the spread of EHW systems.

Additionally, research may focus on the search of potential applications and the extension of the EHW paradigm to different fields, studying its behavior under different circumstances. In order to extend EHW to applications with tight timing requirements, such as real-time processing systems, it becomes necessary to optimize its time behavior, both in terms of data throughput and reduction of the overall training time. 
Finally, it would be profitable to research on the possible improvements on the computational capabilities of the system, studying ways to improve the quality of evolved circuits and the scalability of EHW systems. 



\section{Chapter}

\section{PRELIMINARY DEVELOPMENT OF A DPR-BASED EVOLVABLE HARDWARE SYSTEM}

This chapter describes an EHW system that was built as a proof-of-concept to demonstrate the capabilities of EHW. The development of this system was a prior work to this thesis carried out as part of the author's B.Sc./M.Sc. final project [Mora'11], and upon which most of the work of this thesis has been built.

The system implemented an evolvable image filter that removes noise from grayscale images. The work is based upon the idea of using a systolic array topology to implement EHW, as is described in [Salvador'15]. This platform has been used as a physical realization of such a system in order to prove its feasibility and obtain experimental results.

Additionally, the system has served as a proof-of-concept to implement the dynamic partial reconfiguration techniques described in [Otero'14].

The implementation details of this system are presented in section 3.1. This implementation is evaluated experimentally in section 3.2, where it is seen that the EHW system succeeds in generating configurations able to filter noise from an image.

In section 3.3, different properties of the system are further evaluated in order to assess its behavior as EHW: generalizability, meaning that a result obtained by training with a certain data set can be used with other data sets as well, and adaptivity, showing that the system adapts to different problems rather than a single one, and that the solutions for each problem are specialized for said problem. Additionally, several other beneficial properties such as fault tolerance [Salvador'11b] and scalability [Gallego'13c] are discussed as well. These properties make the system useful for a wide range of applications, some of which are described in section 3.4.

However, as it will be seen in section 3.5, although this proof-of-concept demonstrates the feasibility of such a partially reconfigurable system to implement EHW, the development is far from being applicable to generic real life problems. In the development of this proof-of-concept system, a lot 
of manual work was required, and moving to a different application or even a different technology would require redoing most of it. This hinders the applicability of this theoretical work on practical projects.

This last section motivates the need for a development methodology that simplifies the design of such EHW systems.

As stated before, this chapter describes prior work that was carried out before this thesis; the majority of the contributions of this thesis will appear in chapters 4 to 7 .

\subsection{DESCRIPTION OF THE PRELIMINARY SYSTEM}

The EHW system was implemented on a Xilinx University Program XUPV5LX110T development board [Xilinx-XUPV5]. This board is equipped with a Xilinx Virtex®-5 LX110T FPGA. The choice for this board and FPGA was motivated by the fact that the reconfiguration details for the Virtex- 5 family are extensively documented.

As it has been said, the system implemented an SA-based evolvable image filter whose mission was to remove noise from images. The filter input is an 8 -bit grayscale image with a size of $128 \times 128$ pixels stored in an internal FPGA memory element known as block RAM (BRAM).

The filter uses a $3 \times 3$ pixel sliding window that sweeps the input image line by line and pixel by pixel (Figure 3-1), so that one pixel and its 8 neighbors are fed to the SA in each clock cycle.

The output of the filter is a $126 \times 126$ pixel image that gets stored into another BRAM. In order to compute the fitness of a specific solution, this output is compared pixel by pixel with a reference $126 \times 126$ pixel image in hardware. The resulting fitness is computed as the sum of absolute errors (SAE):

$$
\mathrm{SAE}=\sum_{i=1}^{126} \sum_{j=1}^{126}\left|\mathrm{out}_{i, j}-\operatorname{ref}_{i, j}\right|
$$




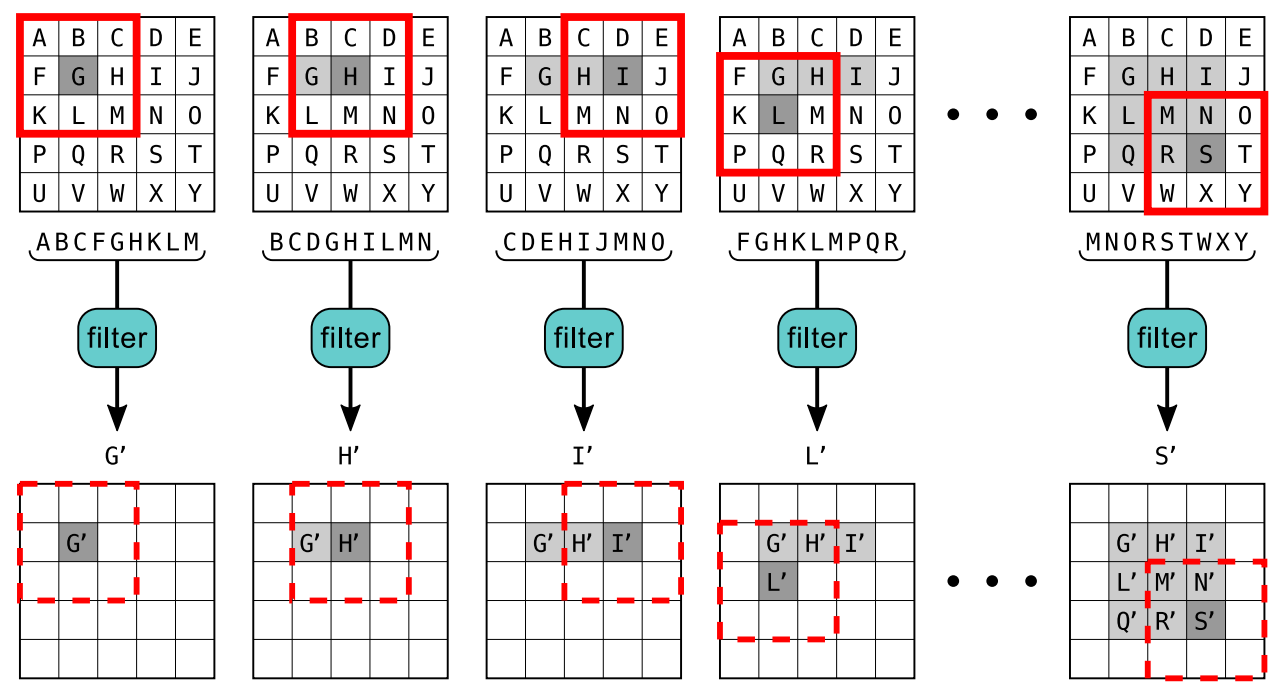

Figure 3-1: Sliding window

with lower values indicating a higher similitude between the output and reference images, and thus a better fitness. ${ }^{1}$

It should be noted that image processing literature often uses a different metric for the visual similitude between images: the peak signal-to-noise ratio (PSNR), which is obtained from the sum of squared errors (SSE) following a logarithmic formula. Thus, a better approach to obtain an optimal PSNR would be to use SSE rather than SAE as the metric to optimize. However, computation of the squared error has a much higher resource usage than the absolute errorspecifically, it requires using DSP blocks, which are scarce in the FPGA-; and previous work [Vašíček'09] has shown that SAE is good enough to drive the EA, achieving similar results to those obtained using SSE (Figure 3-2 ${ }^{(2)}$ ), since both metrics guide the evolution towards minimizing the error.

The whole filtering system is implemented in the FPGA. This system is managed by a MicroBlaze ${ }^{\mathrm{TM}}$ soft-processor, which is implemented in the FPGA as well. The MicroBlaze is in charge of performing the evolutionary algorithm and managing the reconfiguration of the SA. The system is completely autonomous, not requiring an external entity such as a computer to perform the reconfiguration or run the EA.

\footnotetext{
${ }^{1}$ Using a fitness measure where low fitness values indicate a good fitness and high values indicate a bad one may be somewhat counter-intuitive. Nevertheless, although the opposite convention is more frequent in the literature, the current one is not uncommon, and is sometimes referred to as cost function.

${ }^{2}$ These results were obtained with a more mature version of the system, described in $\S 5.3$.
} 


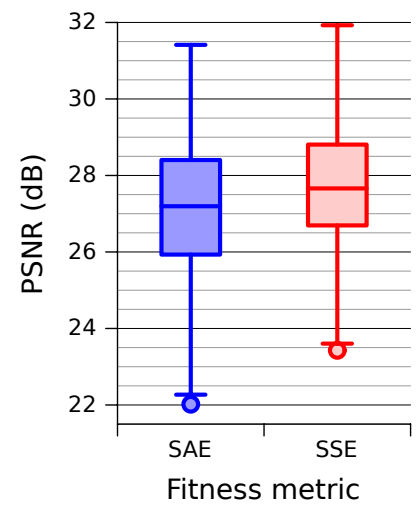

Figure 3-2: Experimental results of the PSNR at the output of an EHW system obtained by evolving using SAE or SSE as fitness metrics (higher is better). As it can be seen, although the usage of SSE as fitness metric leads to slightly better PSNR results, the difference is small, and does not justify the resource overhead introduced by a squared error comparator.

\subsubsection{Systolic array characteristics}

The systolic array had a size of $4 \times 4$ processing elements, as seen in Figure 3-3. Each of these processing elements has two input ports on the north and west borders, and two output ports on the east and south borders. These ports connect each PE with a neighbor PE, or a primary input or output of the SA.

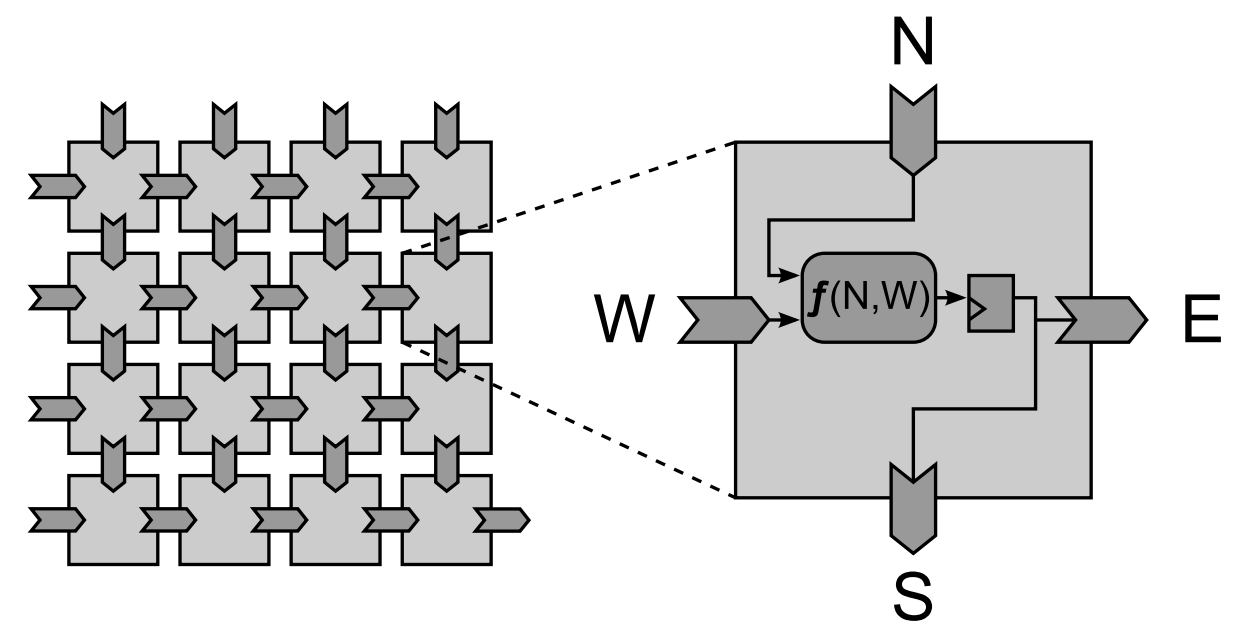

Figure 3-3: $4 \times 4$ systolic array (left) and detail of each PE (right)

Each PE performs a simple arithmetic operation per clock cycle using the data obtained from the north and west inputs-one or both of them depending 
on the operation-, and sends the result to the east and south outputs. ${ }^{3}$ The output is registered in order to obtain a pipelined architecture.

The arithmetic function performed by each PE is fixed during the whole filtering process, not depending on the window position on the image, and is one of the functions listed in Table 3-1 [Salvador'11a]. These functions are based on the ones used in [Vašíček'07b], after removing some of them (mostly bitwise ones) in order to reduce the list to 16 functions. ${ }^{4}$ Each of the primary inputs of the SA is tied to a fixed pixel of the $3 \times 3$ pixel input window. The set of functions for each of the PEs and positions of each pixel within the window constitute the filter configuration, which will be adjusted with the EA described in $\$ 3.1 .3$.

It is worth noting that the input window is injected into all of the primary inputs simultaneously, even though they have different propagation latencies to the array output. Other works [McCanny'82, Sekanina'03] correct this latency discrepancy by adding extra registers on the inputs; however, simulation experiments performed prior to the design of the system showed that these registers can be omitted without a negative impact on the filter performance, as the EA is able to overcome this issue. Nevertheless, this design decision will be revisited in $\$ 5.3 .3$.

\subsubsection{Reconfiguration methodology}

The selection of the pixels from the input window for each primary input is done using multiplexers. Thus, configuration of these inputs is performed by changing the value of a register via a software interface.

Configuration of the PE functions, on the other hand, is done via DPR, as implementing all possible functions and choosing between them with a multiplexer (as is done in VRC) would be too resource wasteful.

In order to perform DPR, modern Xilinx FPGAs provide access to the internal configuration memory through the internal configuration access port (ICAP). In order to access this port, the vendor provides the XPS HWICAP IP core [Xilinx-DS586], which provides a software interface to access this port. However, due to the bottleneck caused by the software interface, usage of this

\footnotetext{
${ }^{3}$ Therefore, although east and south are conceptually two separate ports, their value is the same.

${ }^{4}$ Although the list in [Vašíček'07b] only has 16 functions, the implementation presented here also requires encoding the choice of arguments as part of the function (e.g. $a+b$ may be $N+W$, $N+N$, or $W+W)$, increasing the count to 44 .
} 


\begin{tabular}{ccl} 
Index & Equation & Description \\
\hline 0 & $N+W \bmod 256$ & Addition (modulo 256) \\
1 & $N+N \bmod 256$ & Multiply by 2 (modulo 256) \\
2 & $W+W \bmod 256$ & $\prime$ \\
3 & $\min (N+W, 255)$ & Addition (limited at 255) \\
4 & $\min (N+N, 255)$ & Multiply by 2 (limited at 255) \\
5 & $\min (W+W, 255)$ & " \\
6 & $\left\lfloor\frac{N+W}{2}\right\rfloor$ & Average (rounded down) \\
7 & 255 & Constant value of 255 \\
8 & $\left\lfloor\frac{N}{2}\right\rfloor$ & Divide by 2 (rounded down) \\
9 & $\left\lfloor\frac{W}{2}\right\rfloor$ & " \\
10 & $N$ & Identity (pass through) \\
11 & $W$ & " \\
12 & $\max (N, W)$ & Maximum \\
13 & $\min (N, W)$ & Minimum \\
14 & $\max (N-W, 0)$ & Subtraction (limited at 0) \\
15 & $\max (W-N, 0)$ & "
\end{tabular}

Table 3-1: Functions implemented by the PEs

IP core greatly slows down the reconfiguration. For this reason, the more efficient SDRAM-based HWICAP described in [Otero'10] was used instead.

This IP core fetches partial reconfiguration data from the external DDR2 SDRAM memory in the XUPV5 board and writes it on the ICAP. These fragments of reconfiguration data, known as partial bitstreams (PBS), are stored in a CompactFLASH memory and loaded into the SDRAM at the beginning of the software execution. 


\section{Usage of bus macros for DPR with relocation capabilities}

Xilinx tools include a toolchain for designing DPR-based systems named the partial reconfiguration flow (PR flow). This toolchain allows specifying certain regions of the design to have multiple possible circuits configured on them, and thus could be used to configure individual PEs in the design.

However, PR flow has an important disadvantage: it does not allow relocation, this is, the possibility of using a single PBS on different regions. Therefore, using this flow would imply obtaining a PBS of each function for each of the PE positions, resulting in a PE library as many times larger than necessary as PE positions there are. This would become an impediment for scalability, causing the PE library to grow if larger SAs are desired (as shown in Table 3-2 for a 16 function library with an individual PBS size of $11.5 \mathrm{~KB}$ ), and making dynamic scalability (\$ 3.3.4) impossible.

\begin{tabular}{ccc} 
SA size & \multicolumn{2}{c}{ Library size (MB) } \\
& With relocation & Without relocation \\
\hline $2 \times 2$ & 0.18 & 0.72 \\
$3 \times 3$ & 0.18 & 1.62 \\
$4 \times 4$ & 0.18 & 2.88 \\
$5 \times 5$ & 0.18 & 4.50 \\
$6 \times 6$ & 0.18 & 6.49
\end{tabular}

Table 3-2: PE library size with and without relocation

Given the homogeneity of the internal FPGA fabric, it should be possible to use the configuration data for a certain region on a different one, as long as both regions are structurally identical. Therefore, in order to take advantage of this feature, a different methodology other than the PR flow must be used.

The main challenge when designing a DPR system is ensuring that the interconnections between the reconfigurable region and the static region are compatible. Unfortunately, there is no guarantee that these interconnections will have the same routing for different circuits, even if the reconfigurable circuit is constrained to a certain region of the FPGA.

A widely used solution for this problem is the usage of bus macros [Lim'04, Lysaght'06], which are dummy circuits with a fixed routing (Figure 3-4). These circuits implement a pass-through function and act as a bridge between the static and the reconfigurable regions, using logic elements on both sides 
in order to anchor the interconnections. That way, as long as all versions of the circuit to be reconfigured on a region contain these bus macros on the same positions, the interconnections with the static region will be compatible.

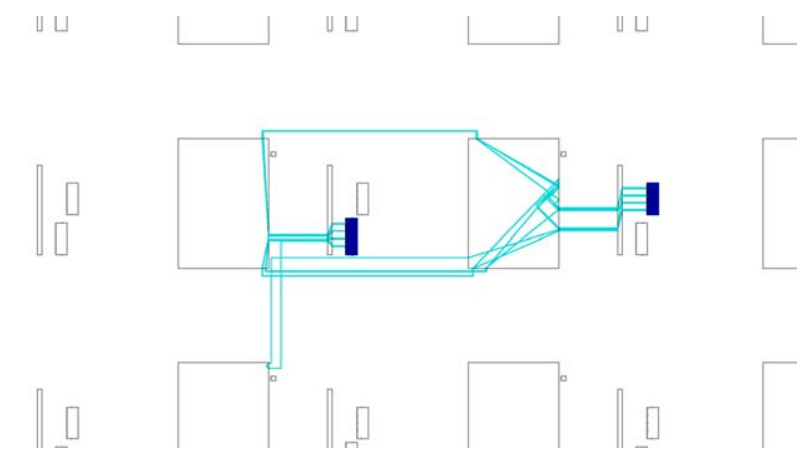

Figure 3-4: 8-bit bus macro connecting two slices (dark blue), as seen in FPGA Editor. The routing of the nets (teal) is fixed.

Additionally, since bus macros are not restricted to a single position but can be placed on different zones of the FPGA, they can be used as a way to obtain relocation, by setting up a grid of bus macros as shown in Figure 3-5.

Another challenge of reconfigurable designs is ensuring that the logic and interconnection elements of both static and reconfigurable regions do not use resources from the neighboring regions; otherwise, the circuits on these regions would become incomplete after reconfiguring.

This is easy to do for the logic resources, as the synthesis tool allows using placement constraints so that logic elements for both the static and reconfigurable regions stay in the corresponding regions. However, this is not so easy to do for the interconnection nets: it is possible that a net belonging to the static region is routed across a reconfigurable region, so when this region is reconfigured, a fragment of the net will be deleted. Similarly, if a net from a reconfigurable region uses routing resources from outside of that region, its PBS will not contain the complete net, and thus will not be fully functional.

The methodology used to overcome this problem is to restrict the placement of logic elements in the static part (for example, avoiding to put two interconnected logic elements on opposite sides of the reconfigurable region, which would likely cause the routing engine to route the interconnection net across said region), followed by a manual inspection in Xilinx FPGA Editor to ensure that no nets violate the region boundaries, and rerouting the nets that do. 

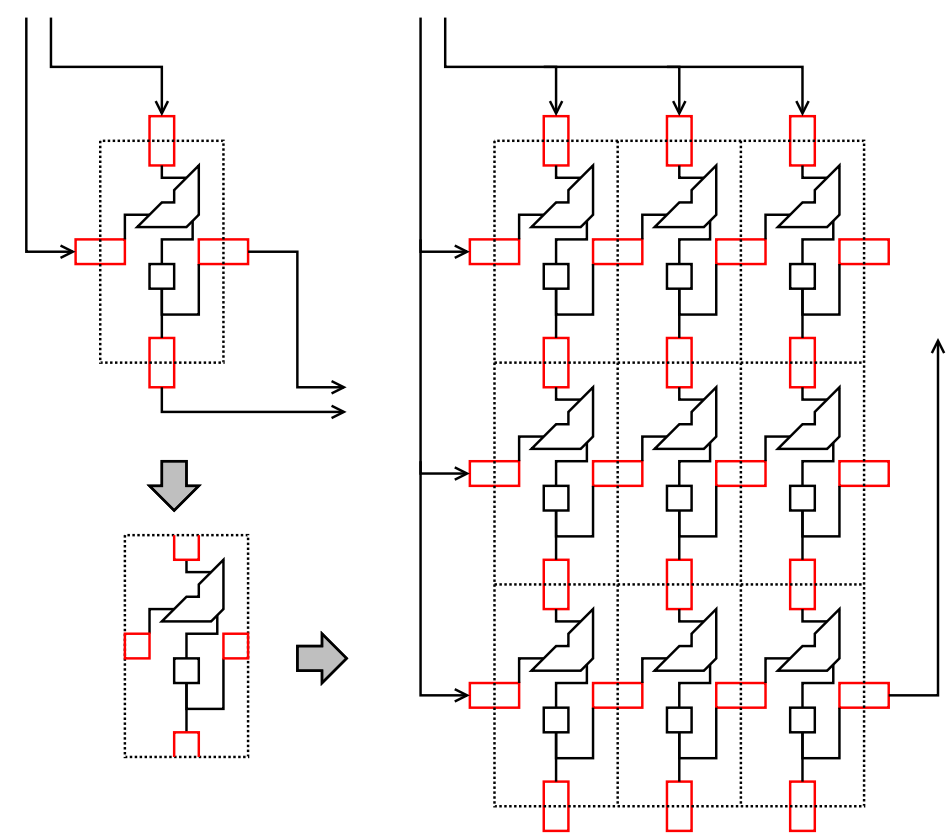

Figure 3-5: Construction of a bus macro based SA. Top left: a PE is designed using horizontal and vertical bus macros (in red) to cross the border of the reconfigurable region (dotted line). Bottom left: the PBS corresponding to the reconfigurable region is extracted. Right: several PBSs are tiled; bus macros ensure that the interconnections will be compatible.

\section{Reducing the size of PEs by using sub-frame reconfiguration}

The aforementioned methodology was used to develop an initial PE library with 16 PBSs for the PE functions described in Table 3-1. However, these PEs have been oversized in order to avoid routing problems, resulting in a size of 20 CLBs high and 2 CLBs wide. This vertical size of 20 CLBs is used because it is the vertical size of a reconfiguration frame, which is the smallest unit of configuration data that can be configured via the ICAP (see Figure 3-6). Nevertheless, these PEs are quite resource wasteful, as each PE uses less than $15 \%$ of the slices in that area.

In order to allow reconfiguring regions smaller than 20 CLBs in height, the reconfiguration engine described in [Otero'10] featured a readback capability where it could read the configuration of a whole frame or set of frames and then write it back replacing a fragment of the data within each frame. This allowed reducing the size of each PE to $5 \times 2$ CLBs, shrinking the size of the array by a factor of 4 . This permitted making larger arrays [Gallego'13c] or putting multiple arrays in parallel [Gallego'13b]. 


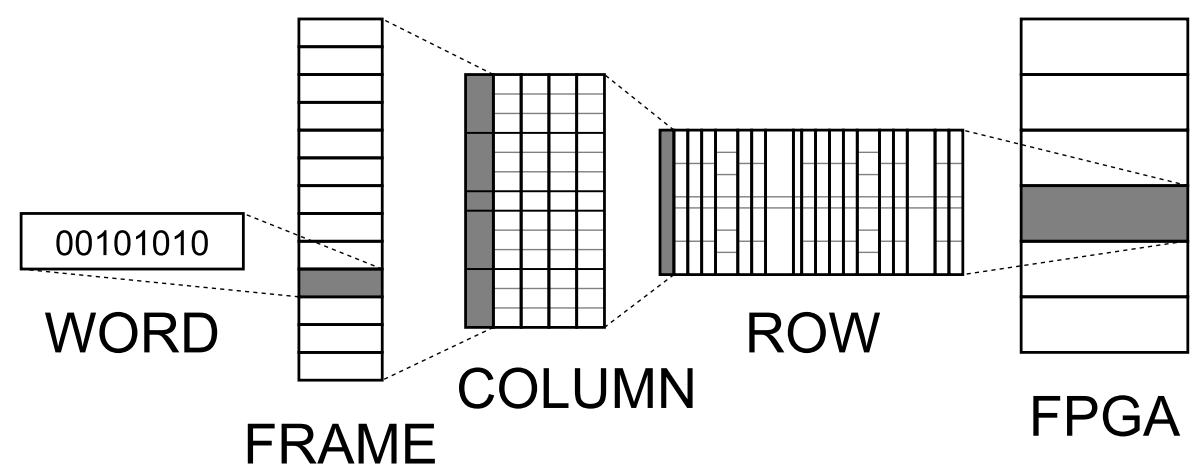

Figure 3-6: Structure of the FPGA configuration memory. A Virtex-5 CLB column contains 20 vertically arranged CLBs.

\section{Using the DREAMS tool for an automated routing and a more efficient resource usage}

One problem of bus macros is that they require using extra logic resources, which may be negligible for large circuits but have an important impact on small ones such as the ones used here. Specifically, the 8-bit bus macros used here use 2 slices-one on each end-to anchor the signals. Thus, a single PE with 4 bus macros used 4 slices (one per bus macro) for the inputs and outputs alone.

However, the resources needed to implement the logic function and 8bit register are small, ranging from 2 to 5 slices. Therefore, near half of the resources in a PE were used solely for routing the signals.

This motivated moving to a different approach, consisting in using a custom routing engine, the DREAMS tool [Otero'12c]. This tool provides a routing methodology that allows freezing fragments of each interconnection net so that it will be consistent across different designs. This provides an alternative to the use of bus macros.

As a result, bus macros can be removed from the design, which not only results in a lower resource usage but also in a smaller delay in each net, since the extra LUTs that were previously added in the data path to anchor the net have been removed.

Additionally, this tool allows introducing extra routing constraints, which solve the problem of nets violating the region borders in an automated manner.

Using this tool, the PEs have been further reduced to a size of $5 \times 1$ CLBs. This has allowed making the array reach larger sizes, of up to $8 \times 7$ PEs. Additionally, the tool has allowed to implement a dynamically scalable array [Gallego'13c] 
that will use more or fewer resources (and thus more or less energy) depending on the requirements of the problem, which will be detailed in $\S 3.3 .4$.

\subsubsection{Preliminary evolutionary algorithm}

Although the system has been designed specifically for image filtering applications, aside from the window-based architecture and the PE function library the design makes no assumptions regarding the task it has to perform. In order to adapt the SA-based filter to a certain task, the system must first undergo a training stage in which an optimization algorithm will be used to find the optimal solution to a specific problem.

Given the complexity and non-linearity of the problem, the optimization algorithm chosen is an evolutionary algorithm (EA), as it does not depend on the smoothness of the fitness landscape as much as other algorithms such as hill climbing.

The EA employed is a simplified version of a genetic algorithm (GA). This algorithm encodes all the information of the SA configuration using a chromosome composed of several genes. Each gene is an integer ${ }^{5}$ representing either the function of a certain PE (using the 0-15 indexes shown in Table 3-1) or a pixel within the input window selected by each input multiplexer (from 0 to 8 ), making a total of 24 genes for a $4 \times 4$ SA. Whether a gene encodes information of a PE or a multiplexer and which of them it corresponds to is determined by the position of the PE within the chromosome.

The EA uses a selection method known as $(1+\lambda)$ [Schwefel'77], ${ }^{6}$ in which a population of one parent individual is mutated in order to obtain $\lambda$ children. Unlike typical GA implementations, this EA does not use crossover, relying exclusively in mutation and selection.

The complete algorithm is as follows: first, an initial parent is obtained by configuring a chromosome as an identity (pass-through) filter. ${ }^{7}$ Then, the EA enters an iterative process that repeats for a given number of generations (100000 in this case [Salvador'13]). In each generation, the parent is mutated

\footnotetext{
${ }^{5}$ Typically, chromosomes in GA are represented as a sequence of bits rather than integers, but this is not a strict requirement: GAs using integers or even real numbers exist as well. [Mitchell'98]

${ }^{6}$ This nomenclature is used in [Schwefel'77] to describe a selection method for evolution strategy (ES) in which a $(\mu+\lambda)$-ES describes an EA with a population of $\mu$ parents and $\lambda$ children. Nevertheless, it is often used for other types of EA as well [Bäck'96].

${ }^{7}$ Alternatively, the chromosome may be initialized with a random configuration; however, it was later seen that starting with a pass-through filter could be advantageous in real-time applications such as the one described in $\$$ 3.3.5.
} 
obtaining $\lambda=8$ children. All of the children are evaluated and compared with the parent. If one or more of the children present a fitness better or equal to the parent, the best of them becomes the new parent. Otherwise, the parent is preserved for the next generation. The result of the evolution is the parent at the end of the 100000 generations.

The pseudocode for this algorithm is shown in Algorithm 3-1.

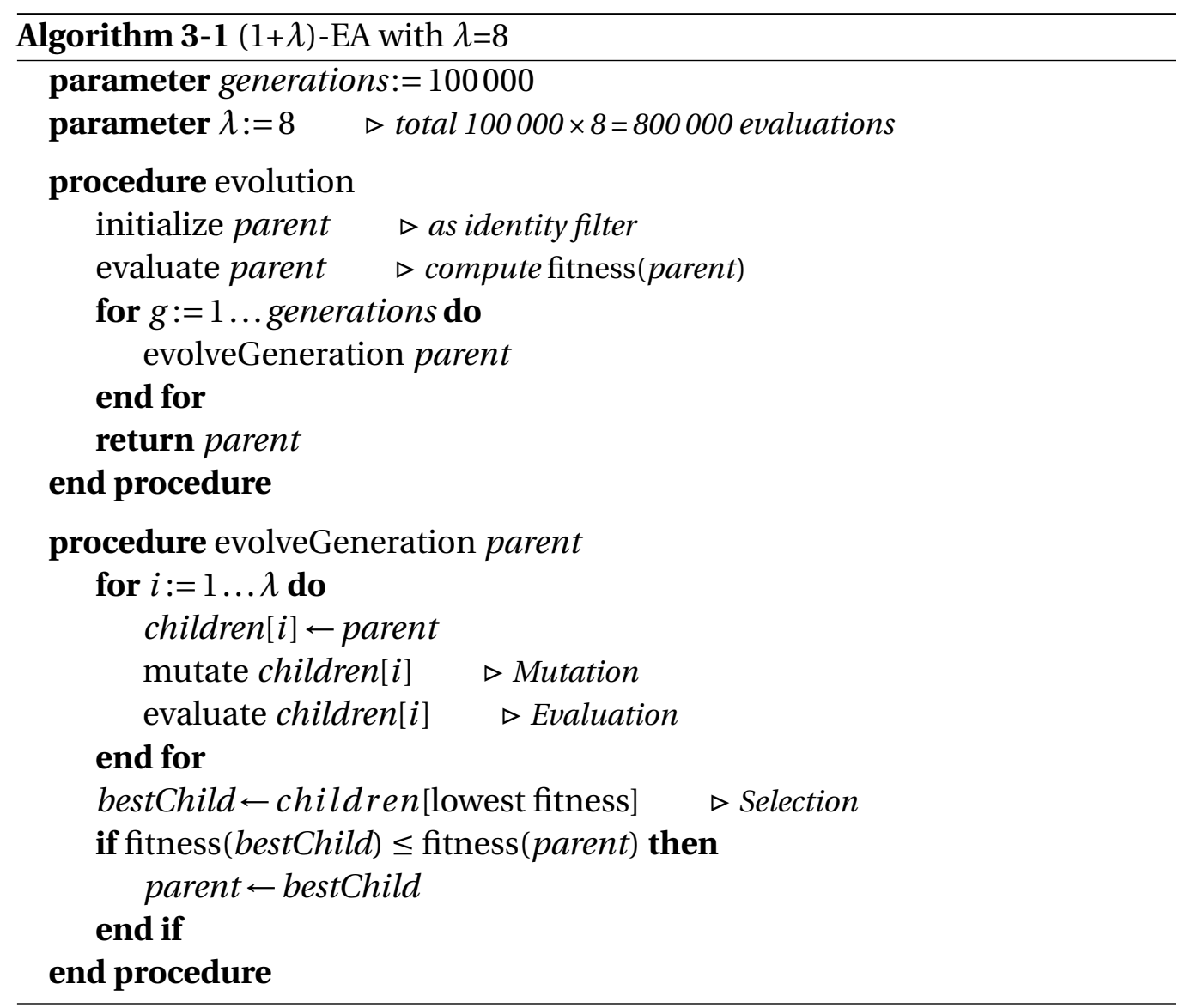

The mutation process consists in selecting a random gene (which will represent either an input multiplexer or a $\mathrm{PE}$ ) and changing its value by a random value in the range of that gene. This process is repeated $K$ times, where $K$ is a fixed parameter of the evolution known as mutation rate. ${ }^{8}$ Experiments have shown that values of $K=3$ yield good results.

The evaluation process consists in implementing a specific configuration from the chromosome into the SA by using DPR to change the functionality

\footnotetext{
${ }^{8}$ In the literature, the mutation rate is often expressed in the form of a percentage; nevertheless, here it refers to an absolute number of genes for convenience.
} 
of the PEs and writing to some registers to change the behavior of the input multiplexers. Then, a $128 \times 128$ pixel image representative of the problem is fed to the filter. The output of the filter is compared with a $126 \times 126$ pixel reference image which demonstrates what the filter should do. The fitness of the solution is defined as the SAE difference between the output and the reference image.

The training input and reference images are generated so that they are representative of the problem. For example, in order to obtain a noise removal filter, the input image will be obtained by adding artificially generated noise to a predefined image, and the reference will be the original image (Figure 3-7).
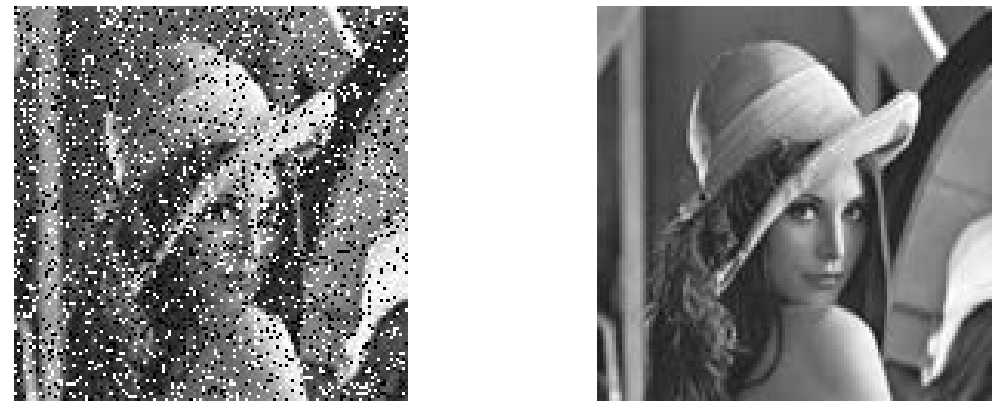

Figure 3-7: Example set of training images that may be used for the evolution in order to obtain a filter able to remove salt and pepper noise. Left: $128 \times 128$ pixel image of Lena, widely used for demonstrative purposes, with a $20 \%$ of artificially generated salt and pepper noise added to it. Right: original image before adding noise, which is cropped to a size of $126 \times 126$ to be used as reference.

\section{Note on the classification of the EA}

The EA in use is not easy to classify as it is inspired in both GA and evolution strategy (ES).

Thus, although the EA is quite similar to a GA, it differs from the latter in the lack of crossover, which is often regarded as the primary operator distinguishing GAs from other stochastic search methods [Mitchell'98, ch.6], in addition to the fact that $a$ single parent is used. For this reason, some authors [Miller'11, Sekanina'13] prefer to classify this kind of EA as an ES.

However, one of the defining properties of ES is the fact that it operates with real numbers, using a mutation operator that consists in adding a randomly generated real number to some of the genes in the chromosome. Additionally, the standard deviation of this random number is also often subject to evolution. This is not the case for the currently used EA, which replaces each mutated gene entirely rather than applying a small variation on it. 
Therefore, this EA is often described as a simplified form of GA [Bäck'96, pt. II]. Nevertheless, given the simplicity of the EA, it is hard to classify it unequivocally.

\subsection{SYSTEM ANALYSIS AND EXPERIMENTAL RESULTS}

Once the EHW image filtering system has been designed and implemented, it is analyzed to assess its performance. This analysis covers both the properties of the hardware (resource usage and operation frequency) and the behavior as an image filter.

All of the results reported here are based on a $4 \times 4$ PE array. ${ }^{9}$

\subsubsection{System characterization}

The hardware has been analyzed in order to obtain reports of the resource usage and the supported frequency of operation. This analysis can be used to compare this solution to others, determine the FPGA requirements to implement such a system, study the integrability of this hardware module into a larger system, and explore optimization and extension possibilities.

\section{Resource usage}

A breakdown of the resource usage of the system is shown in Table 3-3. This table includes the resources present in the area covered by the reconfigurable region, whose size depends on the size of each PE. As it can be seen, the 10 CLBs per PE version uses less than $17 \%$ of the LUT and FF resources available on a Virtex-5 LX110T FPGA. The MicroBlaze-based microprocessing system uses a considerable amount of BRAM, partly due to the controllers for the external CompactFLASH and DDR2 SDRAM needed to store the partial bitstreams.

A resource usage report for the scalable version of the SA using the DREAMS tool is not included because a version of this specific system

\footnotetext{
${ }^{9}$ This small size was chosen due to limitations in the available resources, after determining that this size yielded satisfactory results using a simulation of the hardware. Nevertheless, evolution results with larger arrays (up to $24 \times 24$ PEs) will be evaluated in $§ 5.2$.
} 


\begin{tabular}{lrrrr} 
Part & LUT & FF & BRAM & DSP \\
\hline Static region & & & & \\
SA interface & 1782 & 1597 & 12 & - \\
Reconfiguration engine & 2344 & 2765 & 9 & - \\
Microprocessing system & 5014 & 5825 & 49 & 3 \\
TOTAL & $\mathbf{9 1 4 0}$ & $\mathbf{1 0 1 8 7}$ & $\mathbf{7 0}$ & $\mathbf{3}$ \\
\hline Reconfigurable region & & & & \\
$4 \times 4$ PEs (1 PE = 40 CLBs) & 5120 & 5120 & - & - \\
$4 \times 4$ PEs (1 PE = 10 CLBs) & 1280 & 1280 & - & - \\
\hline \hline Available in Virtex-5 LX110T & 69120 & 69120 & 148 & 64
\end{tabular}

Table 3-3: Resource usage of the system as reported in [Mora'11]. The resources reserved by the reconfigurable region are shown separately.

using that approach has not been implemented. However, a similar-albeit more complex and feature rich-system has been implemented and analyzed by [Gallego'14], showing a similar resource usage for the static system.

\section{Operation frequency}

Xilinx provides a timing analysis tool to obtain an estimate of the maximum frequency at which a system can operate. However, this is a reconfigurable system whose logic and interconnection nets are going to change after each reconfiguration, leading to different timing behaviors that are not covered by such analysis. Therefore, the best way to assess the maximum operating frequency is to do so experimentally.

Once the system was implemented, it was observed that it could operate at a frequency of $200 \mathrm{MHz}$ without causing observable timing errors, which allows filtering a $128 \times 128$ pixel image in $82 \mu$ s. However, the version of the system described in [Gallego'14] presented some signs of timing errors at this speed due to its increased complexity, so the frequency in this version was reduced to $100 \mathrm{MHz}$.

Regarding the reconfiguration engine, the documented nominal frequency of the ICAP is $100 \mathrm{MHz}$, meaning that it can write up to 100 million reconfiguration words per second. Nevertheless, previous work [Claus'10, Hansen'11] shows that the ICAP can be overclocked to much higher frequencies. Thus, the system was implemented with an ICAP frequency of $200 \mathrm{MHz}$, not presenting

\footnotetext{
${ }^{10}$ Excluding the SA itself, which is in the reconfigurable region.
} 
any timing errors. This allows reconfiguring a single $20 \times 2$ CLB PE in less than $16 \mu \mathrm{s}$.

However, this changes when sub-frame reconfiguration is used, needed to reconfigure PEs of a size of $5 \times 2$ CLBs. Somewhat counterintuitively, the number of clock cycles required to reconfigure this smaller $\mathrm{PE}$ is actually larger than what would be needed if the PE were exactly one frame in height, since the configuration data has to be read for the whole frame-tall area and then written back. Furthermore, the readback capabilities of the ICAP seem to be more strict with timing, showing timing errors at $200 \mathrm{MHz}$. For this reason, the ICAP clock has been slowed down to $\mathbf{1 0 0} \mathbf{M H z}$ for designs that use sub-frame reconfiguration. Thus, a single $5 \times 2$ CLB PE takes $64 \mu$ s to be reconfigured.

The MicroBlaze processor supports frequencies of up to $125 \mathrm{MHz}$; however, in order to keep all frequency ratios simple-which has shown to be less prone to errors-its frequency has been set to $100 \mathrm{MHz}$.

With these frequency configurations, the system is able to evaluate $\mathbf{5 7 0 0}$ candidate solutions per second for the $20 \times 2$ CLB version, and $\mathbf{2 6 0 0}$ for the $5 \times 2$ CLB one.

\subsubsection{Evolution results}

In order to evaluate the capabilities of this EHW system as an adaptive image filter, a batch of tests has been run.

These tests have been performed using a Lena image (Figure 3-8), which has been cropped from its original resolution of $256 \times 256$ pixels to $128 \times 128$ in order to fit in the system memory.

The quality of the solutions is measured as the final fitness of the evolution, this is, the SAE between the filtered training input and the training reference.

The specific problem for which the filter has been trained is the removal of salt and pepper noise, in which random pixels of an image get corrupted, being replaced with totally white (salt) or black (pepper) pixels, while the rest of the pixels remain unaltered. Specifically, the filter is trained using an image with a $5 \%$ of salt and pepper noise (5\% of the pixels are corrupted) artificially added to it. This kind of noise has been used often in previous work to evaluate similar adaptive systems [Zhou'98, Saeidi'05, Vašíček'07a].

Each test consists in running the EA described in $\$ 3.1 .350$ times, in order to obtain statistically meaningful results. These 50 runs use identical parameters (run length, number of children, mutation rate), and differ only in the initial random seed. 

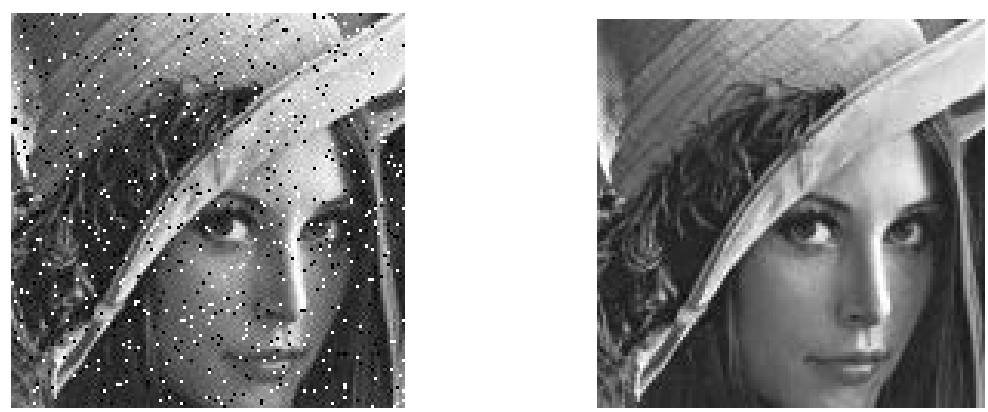

Figure 3-8: Set of training images used for the evolutionary tests. Left: $128 \times 128$ pixel training input, with $5 \%$ random salt and pepper noise artificially added. Right: $126 \times 126$ pixel training reference, which is a cropped version of the original image before adding the noise.

A total of eight tests with mutation rates from 1 to 8 have been performed. The result of these tests is shown in Figure 3-9, where it can be seen that mutation rates between 2 and 5 provide the best results. This is because, as it can be seen in Figure 3-10, a mutation rate of 1 quickly gets stuck at a local optimum and does not improve (the search algorithm is not random enough to get out of this optimum), whereas an excessively high mutation rate leads to an excessively random search, making the algorithm converge too slowly. This has motivated a choice of $K=3$ as the optimal mutation rate for future experiments.

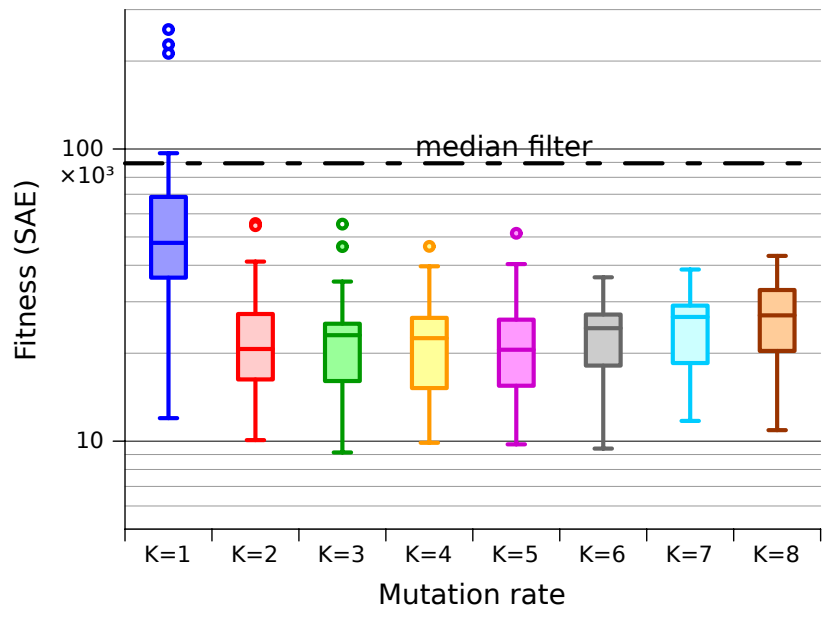

Figure 3-9: Distribution of the evolution results for different mutation rates (SAE; lower is better)

These results include a comparison with a $3 \times 3$ pixel window median filter (a general purpose filter often used to remove this kind of noise), which obtains 

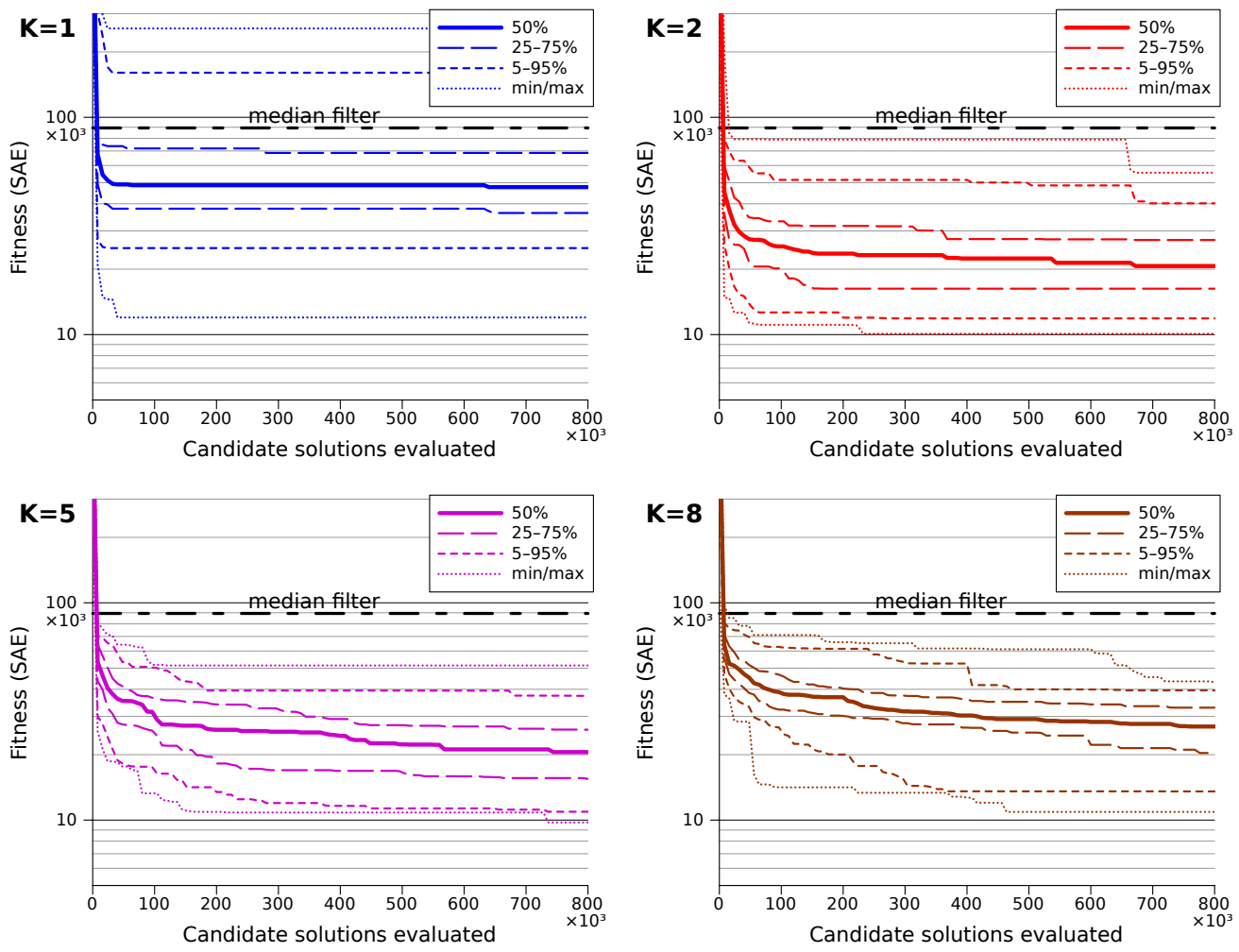

Figure 3-10: Progression of the fitness value during the evolution for different mutation rates, showing the median value of all 50 runs (solid), minimum and maximum (dotted), and different percentiles (dashed).

an SAE of 89345 (black dashed line) for the training image set. As it can be seen, the evolved filters always obtain notably better results than the median filter.

Figure 3-11 shows the final result of some of the trained filters, comparing them with the result of applying a median filter. As it can be seen, these filters obtain visually better results than the median filter, since they do not tend to blur the image as the latter does. 


\subsection{ASSESSMENT OF THE VALIDITY OF THE SOLUTION}

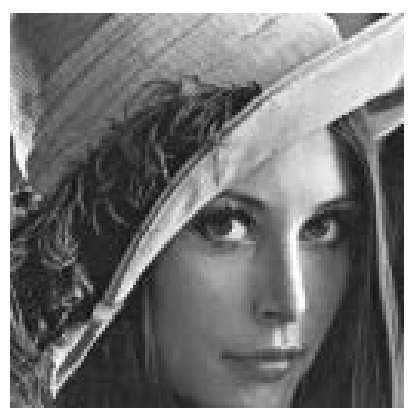

$\mathrm{SAE}=0$ original image

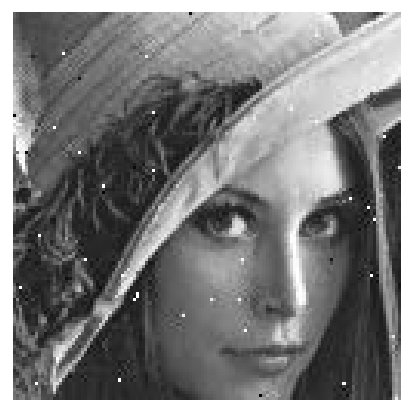

$\mathrm{SAE}=24793$

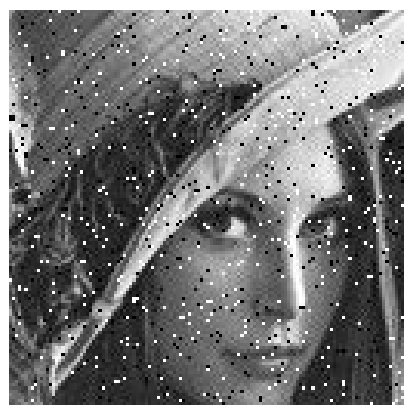

$\mathrm{SAE}=104115$

noisy input

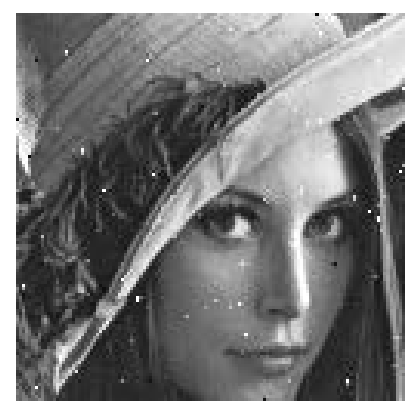

SAE $=22986$

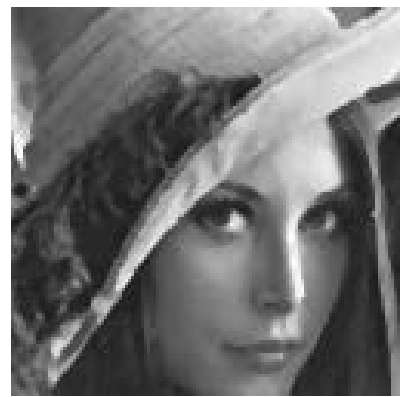

$\mathrm{SAE}=89345$ median filter

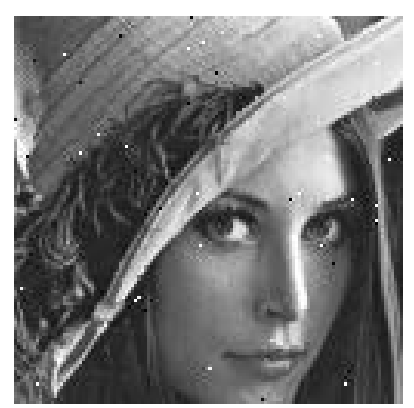

$\mathrm{SAE}=13269$ (best)

output of several evolved filters

Figure 3-11: Some of the results obtained with the evolved filters using a mutation rate of $K=3$ (bottom row) and with a median filter (top right). Although the median filter is more effective at removing all white and black dots, it has the effect of blurring the image details, making the borders less sharp. The best of the 50 results is also shown (bottom right).

\subsection{ASSESSMENT OF THE VALIDITY OF THE SOLUTION AND ANALYSIS OF ITS PROPERTIES}

The analysis performed in $\$ 3.2$ shows that the system can be trained to remove a specific kind of noise from a certain image. However, for this system to be acceptable as an autonomous adaptive filter, some conditions must be met. Specifically, the filter must be able to perform a certain task regardless of the input (generalizability) and the problem conditions (adaptivity). These properties are described and analyzed in this section. 
Additionally, other interesting properties of the system are studied, such as the ability to overcome local defects (fault tolerance) and the runtime scalability of the array. Finally, it is shown how such a system may perform a real time task without the training stage interfering with its operation.

\subsubsection{Generalizability}

A question that often comes up in this kind of adaptive system-and which the reader may be asking - is: "If a single image was used to train the filter, did it really adapt to removing that noise type, or did you just make a circuit that generates that image?" Or, going further: "What is the point of training a filter to remove noise from an image when you already have the original?"

A critical characteristic of evolvable filters is their generalizability, this is, the ability to use a filter trained with a specific data set to process any input. It is possible, however, that such a system specializes excessively on the training data set-sometimes to the point of learning how to produce the training reference regardless of the input-, becoming unable to process any other data. This condition is known as overfitting.

One of the theoretical reasons why this filter is expected to be immune to this condition is its non-locality: this sliding window filter applies the same filtering function to every point of the image and thus the output depends exclusively on the filtered pixel and its surroundings, not its position within the image. Therefore, it is not possible for the filter to "memorize" an image by learning what pixel to generate at each position.

Another way for the filter to "cheat" would be to learn specific patterns and memorize their expected output, so that it specializes on specific segments of the training input. However, the input window is too small for this to happen, and the filter is probably not complex enough to memorize multiple algorithms for each separate segment.

Finally, there is the possibility that the filter adapts only to the features appearing on the training image, so filtering an image with different features would be problematic. For example, if the filter is trained to remove salt and pepper noise from a dark image, it is possible that it does not learn how to filter bright areas, so when this filter is fed an image with bright areas, it is likely that the filter mistakenly treats them as noise and corrupts them (Figure 3-12). For this reason, although the solution is generalizable to an extent, the filter should be trained with an image that is a good enough representative of the problem. 


\subsection{ASSESSMENT OF THE VALIDITY OF THE SOLUTION}
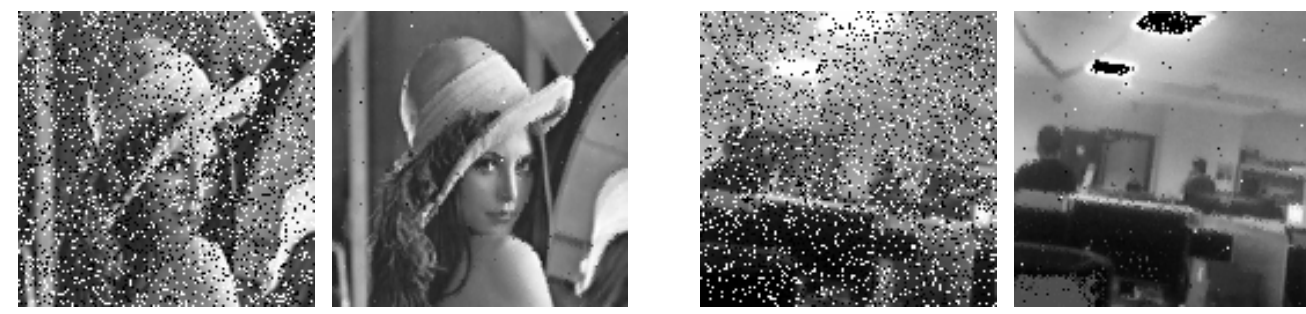

Figure 3-12: Example of overfitting. Left: training input (which has no extremely bright or dark areas) and resulting output. Right: filtering an image with unusual contrast settings produces erroneous results in saturated (black or white) areas.

These theoretical conclusions about the generalizability of the system are experimentally assessed in [Salvador' $13, \$ 5$ ]. As an example, Figure 3-13 shows the result of filtering an image with filters trained for different images. As it can be seen, although each image is best filtered with a filter trained with it, the solutions in all cases are still acceptable, with SAE values about three times smaller than those obtained with the reference median filter.

\subsubsection{Adaptivity}

Another important aspect of this system is its adaptivity, this is, its ability to set up specialized configurations for different problems. This capability is described in [Salvador'13] along with generalizability. Specifically, the ability of the system to adapt to different types of noise, noise levels, and tasks other than noise removal (specifically, an edge detection task) is assessed.

\section{Different types of noise}

Although the aforementioned salt and pepper noise is very commonly used in the literature as an example, it is a relatively easy to filter noise, since corrupted black and white pixels are easy to tell apart from the rest, so the filter could discriminate between corrupt and non-corrupt pixels. Additionally, the sparseness of the corrupt pixels allows estimating their original value from their surroundings.

For this reason, the system has been tested with other types of noise; namely impulse noise, where pixels are replaced with a uniformly distributed random value from 0 to 255, and burst noise, where noise is realized as sequences of corrupt white pixels rather than being scattered over the image, emulating the effect of a faulty transmission line. Additionally, additive Gaussian noise has been considered, where all pixels vary their value slightly, 

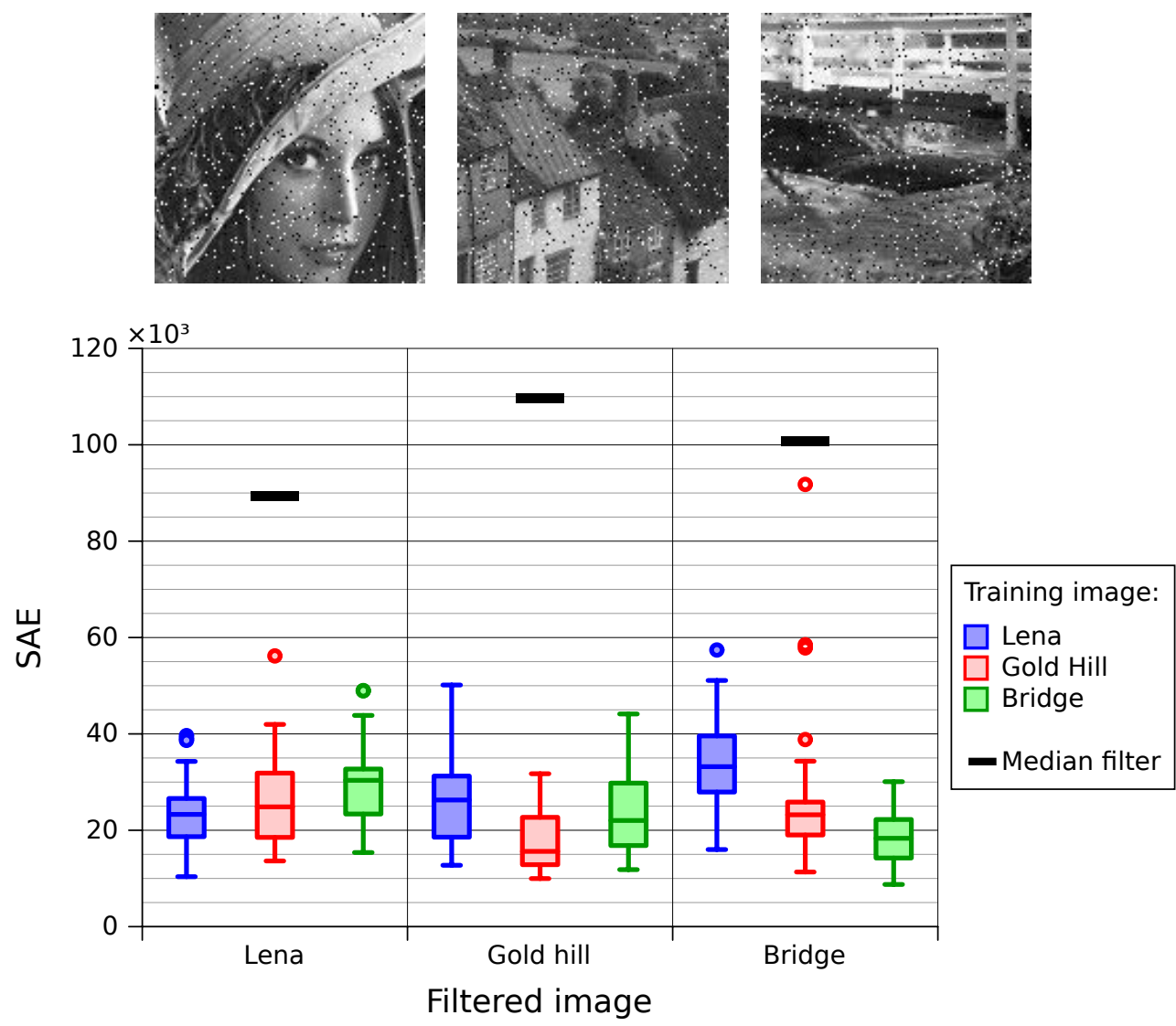

Figure 3-13: Results of filters trained with Lena, Gold hill, and Bridge images (top) when applied to each image. The result of applying a $3 \times 3$ window median filter to each image is indicated with a black dash.

with the error following a normal distribution; however this type of noise is normally considered to be out of the scope of filters based on a sliding window, requiring more complex procedures for a satisfactory result. All the noise types are shown in Figure 3-14.

It has been observed that the filter performs satisfactorily for impulse and burst noise. ${ }^{11}$

The system does not perform as well with Gaussian noise-then again, neither do other window-based filters such as the median filter. In this case, the system adapts by generating a filter that blurs the input image by averaging pixel values, thus removing the heterogeneity introduced by the noise but also

\footnotetext{
${ }^{11}$ Results for different noise types can be seen in [Mora'13b, figs. 11 and 12].
} 

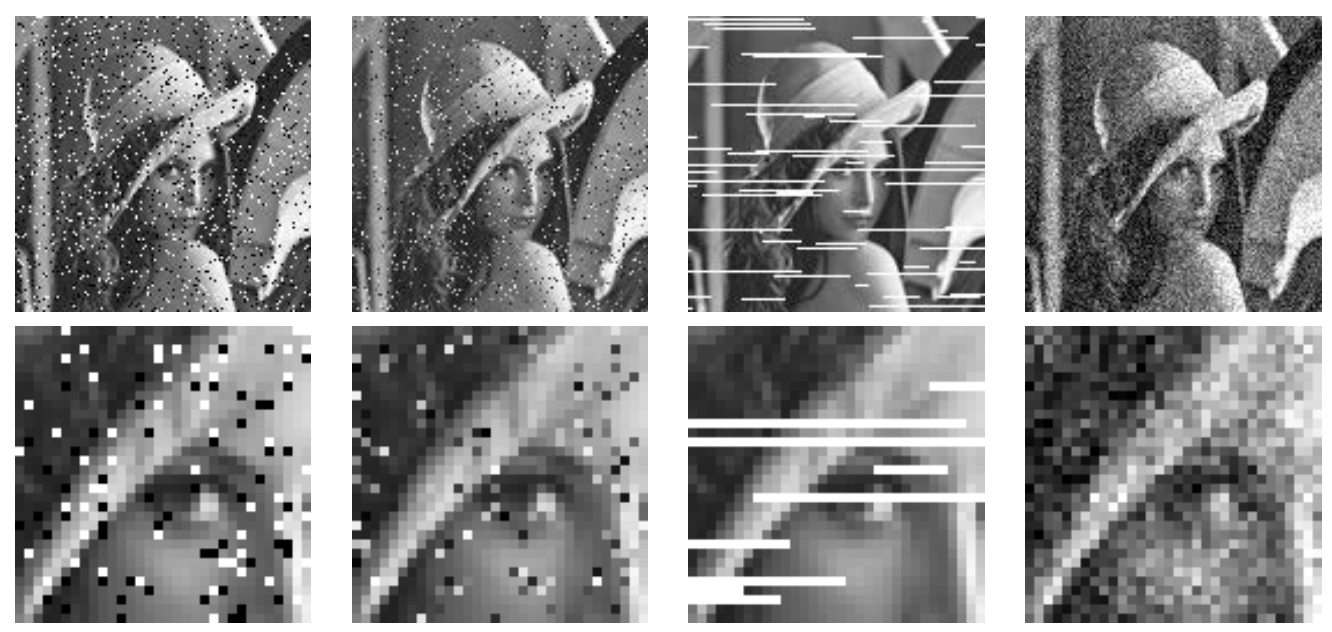

Figure 3-14: Different types of noise. From left to right: salt and pepper, impulse, burst, and Gaussian. Details of each image are included at the bottom.

removing details of the image. A solution for this might be to use larger input windows.

\section{Different noise levels}

As it is described in [Salvador'13], this filter can not only adapt to different types of noise, but also to different levels of noise. Figure 3-15 shows how an image with a certain noise level will be best filtered with a filter trained for that noise level, although similar results are obtained with filters trained for a different level. Specifically, a high-noise image will require a more aggressive filter that may corrupt some details of the image, but for a low-noise image a more conservative filter will still do a good job at removing the sparse noise while respecting the details, as can be seen in Figure 3-16.

As it can be seen, performance is similar to that of a median filter for high noise levels, but for low levels the system is able to find optimized solutions that outperform the median filter. 


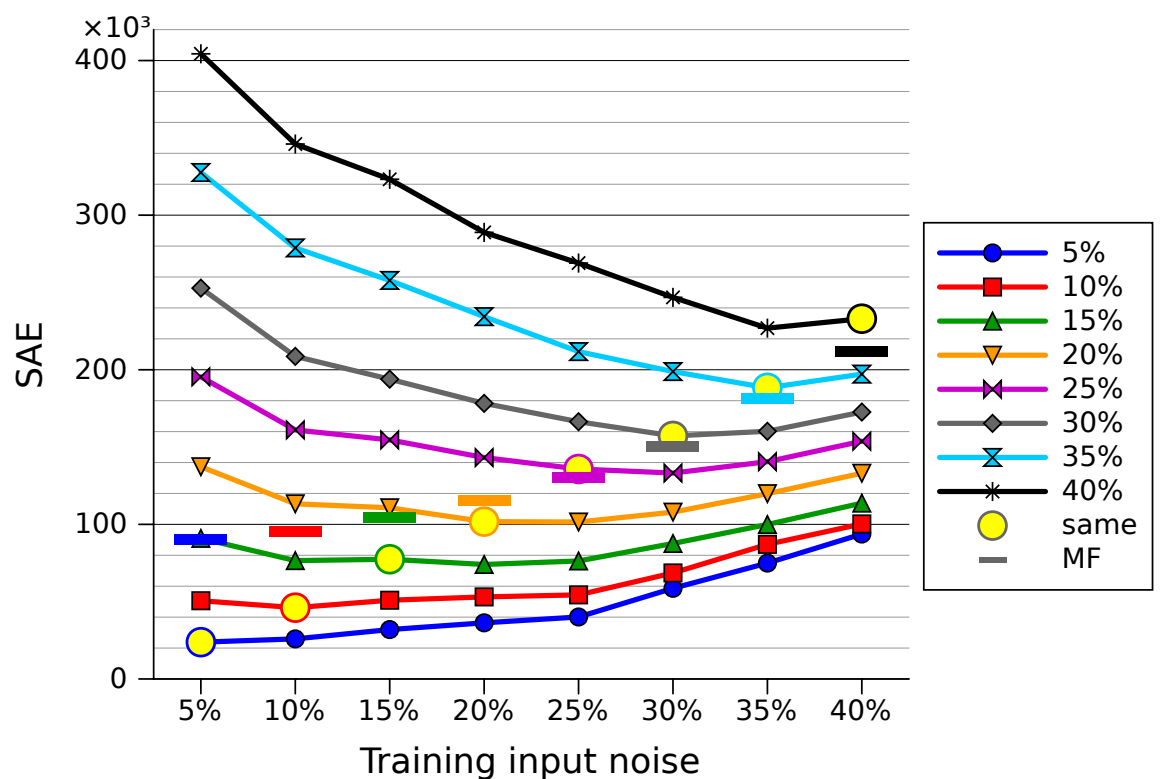

Figure 3-15: Median value of SAE obtained when filtering images with noise levels from $5 \%$ to $40 \%$ with filters trained for different noise levels. Results obtained with filters trained for the same noise level are marked with a yellow circle. The result of applying a $3 \times 3$ median filter to each image is indicated with a dash.

\section{Different tasks}

In addition to noise removal, this system also performs nicely as a hardware accelerator for other image processing tasks such as edge detection. For this application, the system is trained using a clean image at its input and the result of applying the desired window-based filter in software. This can be useful when this software implementation of the filter is available but consumes an excess amount of time.

Additionally, combined tasks such as noise removal and edge detection have been successfully tested [Salvador'13, fig. 9]. This is a complex task since edge detection is very susceptible to noise, so it is expected that the results may improve using larger or cascaded filters. 


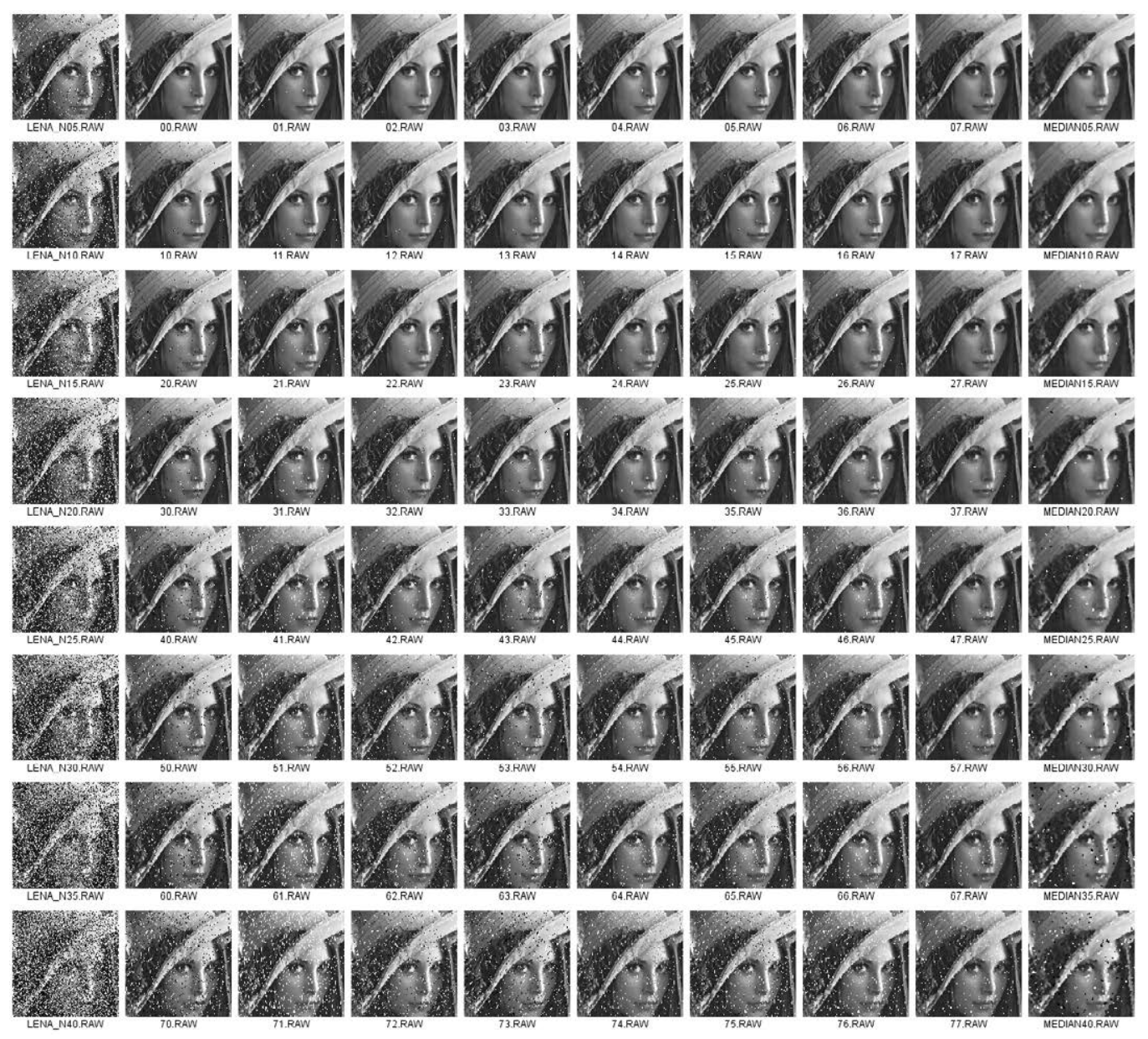

Figure 3-16: Result of filtering images with noise levels from $5 \%$ (top row) to $40 \%$ (bottom row) with filters trained for different noise levels. Left column is the input image; the following columns are the results of filtering each image with filters trained for different noise levels (from 5 to $40 \%$ ). The rightmost column shows the result of a median filter.

\subsubsection{Fault tolerance}

One of the advantages of intrinsic evolution-based EHW is the ability to adapt to faults in the circuit fabric itself, since in case part of the circuit fails, the EA can find an alternative solution that avoids the use of that part.

In the case of an SA implemented on an FPGA, multiple conditions (degradation of the FPGA, impact of a high energy particle...) could cause a permanent fault on a certain PE. If this happens, the EA can be run again in order to find a solution adapted to the new situation. 
However, in the implementation described so far, the bottom right PE becomes a critical point of failure, as it is a bottleneck for the data: if this element fails, the output will be "blocked". This can be solved by adding a multiplexer at the output that allows selecting any of the east outputs of the SA (Figure 3-17). ${ }^{12}$
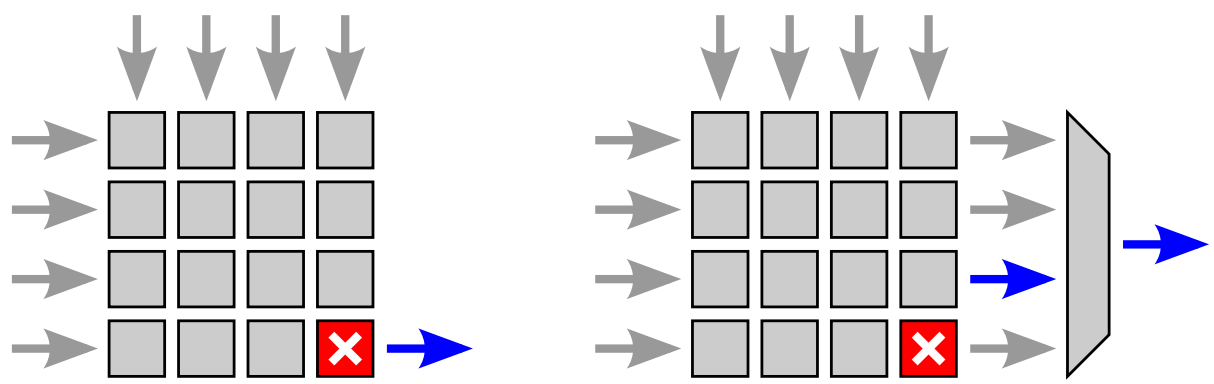

Figure 3-17: Left: with no output selector, the bottom right $P E$ becomes a failure point, blocking the output (blue arrow). Right: if an output selector is added, the system can overcome a failure on the bottom right PE by selecting a different output.

This addition introduces a new problem: since each PE is registered, the latency of a filter depends on the length of the predominant ${ }^{13}$ data path on the filter, which is not known at design time. ${ }^{14}$ Under the previous considerations, the latency was just assumed to be somewhat consistent regardless of the solution, and was given a fixed value of 5 clock cycles as this showed the best experimental results. However, in case of a fault, different paths circumventing the failing PE may show uneven lengths (Figure 3-18), resulting in the latency of the obtained filters having a high variability. Thus, restricting the latency of the filter to a fixed value would exclude some potentially good solutions.

The solution chosen for this problem is to allow the filter have a configurable variable latency. In order to find the optimal latency for a certain configuration, multiple comparators are used in parallel which compare the reference image with versions of the output shifted a number of clock cycles, as shown in Figure 3-19. The hardware peripheral then reports the lowest SAE as well as the latency with which it was obtained, so the system can evolve normally using the lowest SAE as fitness metric and finally selecting the reported latency once the evolution has finished.

\footnotetext{
${ }^{12}$ It is assumed that neither the output multiplexer nor the rest of the logic outside the SA (window generator, input multiplexers, microprocessor...) are vulnerable to faults. This can be achieved with design techniques such as triple modular redundancy, but the implementation and analysis of these are out of the scope of this thesis.

${ }^{13}$ The output of the SA will in fact be a combination of paths with different latencies.

${ }^{14}$ This is a consequence of the decision described in $\$ 3.1 .1$ of not using a strict pipelining, and will motivate its correction in $§$ 5.3.3.
} 


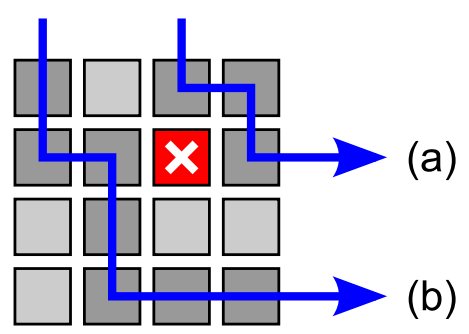

Figure 3-18: With one faulty PE, the latency of the solution will depend on the predominant data path: path (a) has a latency of 3 clock cycles whereas path (b) has a latency of 7 .

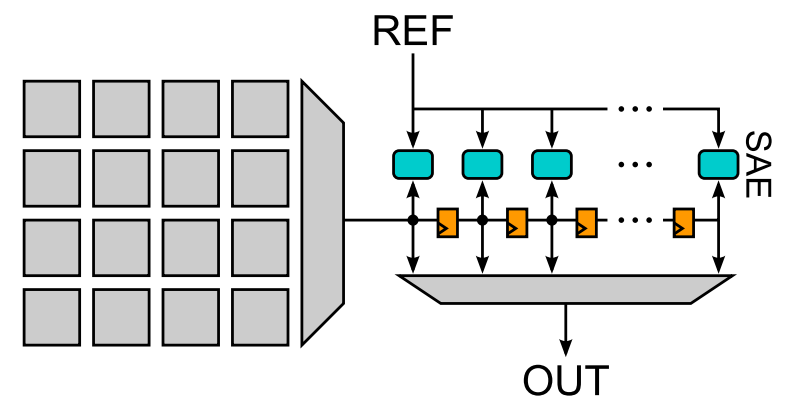

Figure 3-19: Computing of fitnesses corresponding to different latencies using multiple fitness computing units (blue). The reference image must be delayed for as many clock cycles as the largest expected latency; each registering stage (orange) will subtract one clock cycle from the expected latency of the filter in order to match that of the reference image.

Fault tolerance results of the system using this solution are analyzed in [Salvador'11b].

\subsubsection{Scalability}

As it was said in $\$ 3.1 .2$, by using the routing capabilities of the DREAMS tool [Otero' $12 \mathrm{c}$ ] it is possible to easily implement a run-time scalable SA, since this tool simplifies the development of interconnecting PBS and reduces their resource usage.

This requires leaving some unused area on the FPGA so that the array can grow, as well as scalable interconnections at the SA input and output. The latter is solved in [Gallego'13c] by making these interconnections (input and output multiplexers) modular. Multiplexers are replaced with selectors with a fixed 
functionality; changing the input and output configuration is done using the reconfiguration engine.

In order to reduce the number of interconnection nets to be routed on the SA input selectors, it has been originally proposed to only transmit the three rightmost pixels of the $3 \times 3$ window; each selector will be able to obtain an arbitrary pixel by delaying one of these pixels 0,1 , or 2 clock cycles, as shown in Figure 3-20. This allows reducing the number of nets between selectors from 72 to 24 , which simplifies the routing process considerably. Despite of this, routing is still quite complex and results in the selectors being considerably large (10 CLBs, twice as large as each 5 CLB PE).
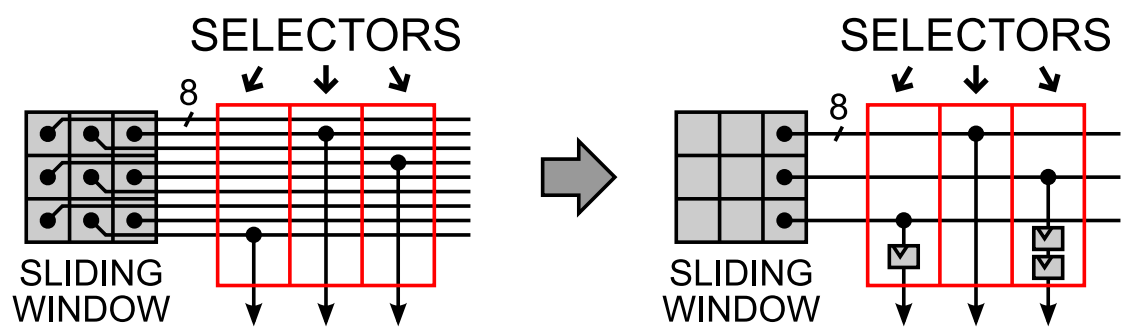

Figure 3-20: Reduction of 9 input channels to 3 combined with additional delay

Original contribution 3-1 The replacement of 9 input channels to 3 plus a variable delay as the primary input for a system based on a $3 \times 3$ pixel sliding window, in order to reduce the number of channels to route. (This is also advantageous in terms of FF usage when these input channels need to be registered, as will be the case in \$5.1.)

\subsubsection{Real-time capabilities}

Finally, it is worth noting that, although the system requires a few minutes to complete the evolution process, this does not mean that it is not suited for real time applications.

In [Mora'14], it is demonstrated how the system can operate in real time starting from a default configuration, using the time between frames to improve the solution. This way, even if the EA needs to be relaunched (for example, because the external conditions have changed or faults have appeared), the system can still work at a sub-optimal performance until a better solution is found. 


\subsection{DERIVED APPLICATIONS}

Based on the functionality of this hardware system, several derived and related projects have been developed in order to demonstrate and evaluate its capabilities and study possible applications of such a system.

\subsubsection{CPU and GPU implementations}

In order to aid in the development and improvement of the system, an accurate software simulation of the SA has been implemented. This simulation not only helps in the development of the system, but can also be used in parallel heterogeneous systems that distribute the evolution process across different platforms, such as FPGAs and PCs.

However, given the sequential nature of a software implementation versus the parallel nature of FPGAs, this simulation is considerably slower than the hardware implementation, even despite of PCs working at a much higher frequency. For this reason, the simulation platform has been also implemented on a GPU using the general-purpose computing on GPU (GPGPU) languages CUDA [Fernández'14] and OpenCL [Niño'15].

This varied implementation would allow a cooperative network of heterogeneous devices (FPGA, CPU, GPU) to perform a distributed evolution in parallel. However, this has been left for future work.

\subsubsection{Realistic noise effects and extension to color images}

One of the problems of the described implementation is its limitation to a few types of noise.

In [Niño' 15], it is justified that real life applications would require filtering more complex types of noise, such as additive white noise, and an SA-based application is designed in order to deal with these types of noise. This is achieved by combining the original input with the output of an SA-based filter, to a degree determined by an SA-based noise quantifier that determines the required intensity of the filtered signal for each pixel. Additionally, a set of new PE functions are proposed and evaluated.

Furthermore, the aforementioned work analyzes the effect of correlated and uncorrelated noise on multiple channels, which improves the system for its application to RGB images. 


\subsubsection{Exploiting scalability for increased fault tolerance}

Given the reduced size of PEs-specially when making use of the DREAMS tool as described in $\$ 3.1 .2$ - it is possible to make rather large SAs.

This not only allows to generate more complex filters, but also to increase the fault tolerance of the system. In [Gallego' 13c], it is shown how a faulty array can recover more easily by upscaling its size.

Another approach to scalability is the implementation of multiple arrays working simultaneously. This is demonstrated in [Gallego'13b], where three $4 \times 4$ SAs are implemented in such a way that they can be configured to work in parallel, allowing to filter multiple images separately, or in cascade, increasing the potential complexity of a filter. This redundancy in the number of arrays can also be used as a tool for fault tolerance, since a faulty array could be discarded, leaving the system to work with the remaining two.

\subsubsection{Learning by imitation}

As it has been stated in $\S 3.3 .2$, a useful application of this family of adaptive filters is the imitation of preexisting algorithms.

This finds an excellent application in the field of self healing hardware when multiple arrays are available. In [Gallego'13b], a faulty array is re-trained so that it imitates the output of a healthy array, taking advantage of the multiple parallel arrays that have been implemented.

\subsubsection{Real-time scalable demo}

In order to demonstrate the real time and fault tolerance capabilities of the system, a demo was developed (Figure 3-21). ${ }^{15}$ This demo was implemented on a Virtex-5-based XUPV5 development board, displaying on a monitor the evolution results, as well as a noisy video input fetched from a digital camera attached to the board, along with the filtered video output.

A variation of the demo is used to demonstrate the autonomy of the system by having it re-evolve and scale automatically-without user interactionwhen a change in the filter performance is detected.

\footnotetext{
${ }^{15}$ This demo has evolved together with the project, adopting new features as they are developed. The screenshot displayed in Figure 3-21 corresponds to the latest version, which includes optimizations described in chapters 4,5 , and 6 .
} 


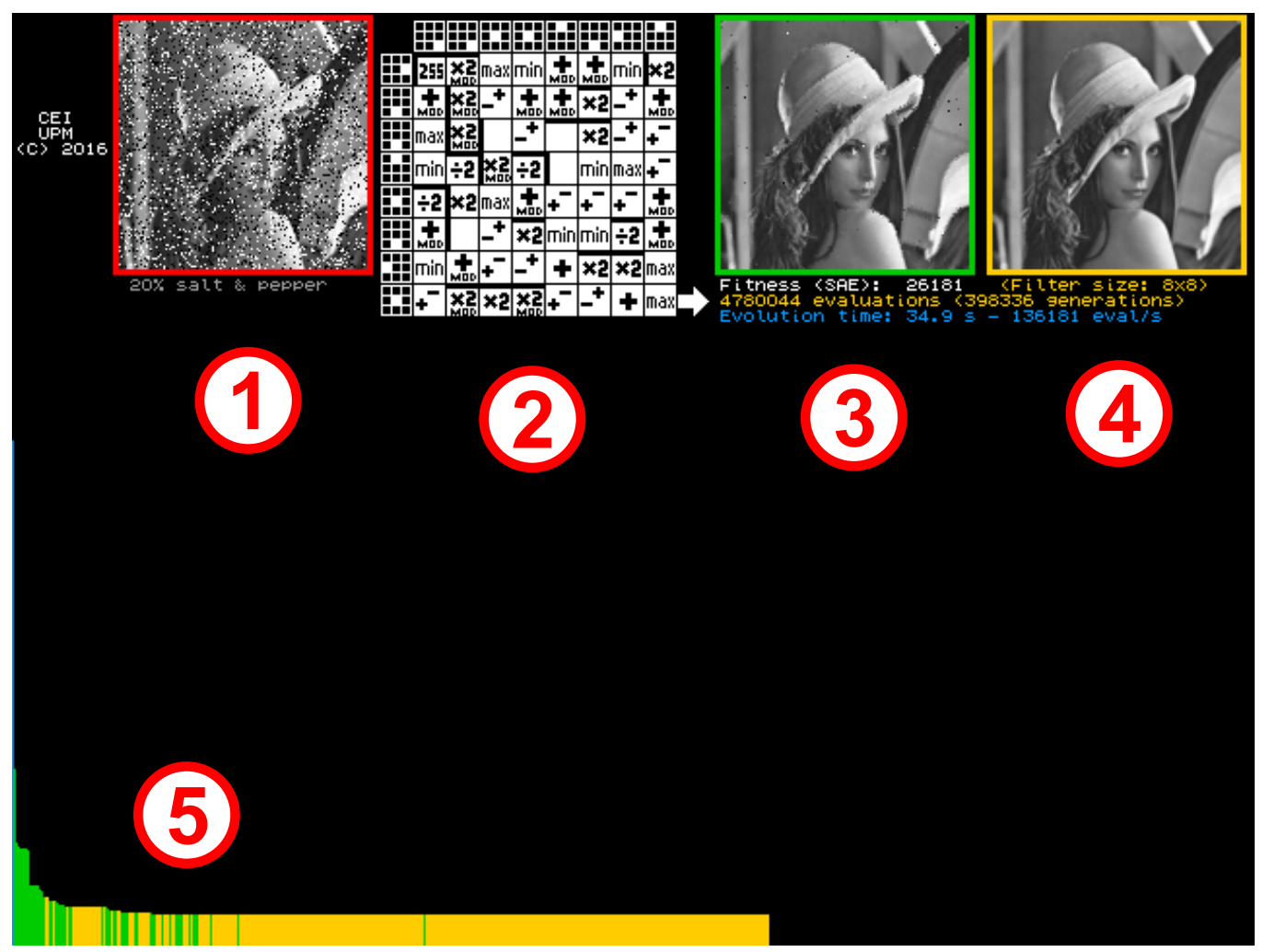

Figure 3-21: Screenshot of the demo, showing how a corrupted image (1) is filtered with the displayed filter configuration (2) in order to obtain an output (3) that matches the reference (4) as accurately as possible. Both filter configuration and resulting output get updated in real time as the evolution progresses. The variation of the fitness is displayed on a plot (5).

Although the development of this demo is not of much academic interest per se, it has been very useful to disseminate the advantages and capabilities of dynamically reconfigurable EHW to the academic world as well as the industrial sector. 


\subsection{PROBLEMS OF THE SOLUTION AND MOTIVATION FOR A CHANGE IN THE DESIGN METHODOLOGY}

The system described in this chapter serves as proof for the feasibility of the proposed implementation as an EHW system. However, this does not provide a general solution for EHW problems: in its current status, it has several weaknesses that difficult its use on other applications.

This section discusses which changes would be necessary to apply to the design methodology used to develop this proof-of-concept system in order to make it generalizable.

\subsubsection{Integration}

The development of this hardware module has focused on a single applicationin fact, the application has been built around the hardware module more than the other way around. This is due to the fact that the system has been developed as a research-oriented proof of concept rather than a general solution.

This does not mean that the hardware developed cannot be reused in other projects: the SA and controlling components could be integrated into a more complete system where the EHW filter is just one of the many parts. However, this would involve redesigning the system, rewriting many of its parts to adapt it to different applications.

Instead of having to do this for every application of this kind of EHW system, it would be desirable to develop a design methodology that simplified the integration of an SA into different designs.

This will be tackled in $\$ 5.3$ by incrementing the parameterization of the module, which will permit its inclusion in new designs with different requirements without modifying the internal structure of the module. 


\subsubsection{Extension}

Another problem of the system is its lack of modularity. This system has been developed targeting a single application, and thus each of its parts has been designed to fit the requirements of that specific application. In general, this is not a good design methodology; instead, it would be preferable to have the system clearly structured into separate modules, each with a clearly defined task.

This modularity is important towards the maintainability of the system; more specifically, to its extension. Separating the system in clearly separate functional modules would simplify the modification of different aspects of the system while keeping the rest intact. Additionally, a modular design will promote the reutilization of its parts for the development of derived applications. Such a modular redesign of the system will be described in $§ 5.3$.

\subsubsection{Speed}

As it was seen in $\$ 3.2 .1$, this EHW implementation is able to test from 2600 to 5700 candidate solutions per second. Since the EA runs for 100000 generations ${ }^{16}$ and tests 8 candidate solutions in each generation, the evolution will need 3 to 5 minutes to complete. This time may be acceptable for some applications, but there may be cases in which a few seconds is already too much.

This evolution time can be reduced in three different ways: by reducing the reconfiguration time (which will be the main goal of $\$ 4.1$ ), by increasing the filter throughput (which will be tackled in $\$ 5.1$ and $\$ 5.3$ ), and by improving the EA so that the same results are achieved with fewer evaluations (for which a series of EA techniques will be analyzed in $\$ 6.1$ ).

\subsubsection{Real-time processing and larger video formats}

The current implementation relies on BRAM memory for temporarily storing the input and filtered images. This is not a problem for the current implementation, where a $128 \times 128$ pixel image is filtered. However, using memory blocks to store the video frames has several disadvantages.

First, the amount of BRAM memory is limited. As an example, the Virtex-5 LX110T FPGA that was used for the project has about $600 \mathrm{~KB}$ of memory, which

\footnotetext{
${ }^{16}$ This number of generations is not a hard requirement and could be reduced at the expense of the quality of the result.
} 
would not be enough to store two grayscale VGA frames $(640 \times 480)$. This could be solved by using the external SDRAM to store the images, but that would complicate the system considerably.

Second, needing to have one complete image before the filtering process can start would severely increase the latency of the system, even more so if multiple intermediate memories are used.

Thus, a better solution would be to move towards a video streaming approach, in which data is processed sequentially without using intermediate memories. An implementation of the system that uses this approach will be the main topic of $\S 5.4$.

\subsubsection{Technology transition}

Finally, one of the major concerns for the future development of this system is more of a technical nature: the FPGA model used, a Virtex-5, is rather old and may be on the way of becoming deprecated, as newer FPGAs are appearing.

Specifically, the 7-series Zynq all-programmable SoC-an integrated system combining an FPGA with an ARM-based SoC-is receiving growing attention. Thus, new projects are more likely to be based on this technology, and therefore limiting the current development to an old FPGA family would decrease its usability in future projects.

Additionally, the software platform used for the design, Xilinx ISE, was discontinued in late 2013. ${ }^{17}$ Its replacement, Vivado, is a promising suite that provides numerous IP cores and facilitates the development of video applications. Nevertheless, the design methodology in this suite is different from that on ISE, and therefore some changes to the IP core would be required.

The design considerations necessary for this technology transition will be described in $§ 5.4$.

\footnotetext{
${ }^{17}$ This suite is still supported and available for download, but no new versions have been released since 2013, except for a 2018 release for Windows 10 supporting Spartan-6 FPGAs only.
} 


\subsubsection{Conclusions}

The system described in this chapter is a very promising proof of concept for DPR-based EHW systems. However, there are several obstacles that hinder its usefulness for future projects.

In the following chapters of this thesis, the different problems enumerated in this section will be tackled in order to ensure the generalizability of the system and its future extension to new projects, not being limited to a single platform, task, or use case. Different techniques will be explored in order to maximize the performance and capabilities of the EHW system and minimize the cost of implementation, both in terms of resource usage and effort of adoption. 



\section{Chapter}

\section{RECONFIGURATION TECHNIQUES: METHODOLOGY AND TOOLS}

This chapter focuses on the aspects of the thesis related to the reconfiguration process, from software tools to assist in the development to the methodology used in the reconfiguration process.

In section 4.1, a new reconfiguration methodology is originally proposed to solve problems posed with former reconfiguration problems, focusing on fine grain reconfiguration.

Section 4.2 describes the methodology developed to obtain the partial bitstreams that will be used in the reconfigurable design. This methodology is not focused exclusively on the hardware architecture described in this thesis, but rather meant as a general purpose and extensible methodology that may be useful in future projects as well. The section concludes by presenting a series of partial bitstream extraction tools that have been elaborated based on this methodology. These tools are currently being used in other projects involving DPR [Zamacola'18].

\subsection{A GENERIC FINE-GRAIN RECONFIGURATION ENGINE}

One problem with the existing flows for DPR is that they typically target the reconfiguration of relatively large areas of an FPGA. However, there are use cases which require modifying small parts of the design, this is, performing fine grain reconfiguration, which is not covered by the aforementioned flows, thus requiring the use of custom DPR tools and methodologies. One such case is that of DPR-based EHW systems, which typically involves the reconfiguration of small-sized PEs. 
Furthermore, one of the keys to achieve good performance in DPR-based EHW systems is minimizing the reconfiguration time. When the training stage of EHW is limited in time (for example, for real time applications), short reconfiguration times allow testing a larger number of candidate solutions, thus improving the chances of obtaining a suitable solution. This limits the suitability of software for driving the reconfiguration process when the reconfiguration data needs to be preprocessed in some way, as may be the case in the aforementioned custom fine grain DPR methodologies.

In this section, the problems with the currently available solutions for finegrained DPR are analyzed, and a hardware implementation of a reconfiguration engine targeting fine-grained reconfiguration is described.

Original contribution 4-1 A generalized DPR engine implemented as a reusable module with a general-purpose interface, which may not only be useful for the SA-based system studied in this thesis, but also for any other fine-grain DPR application.

\subsubsection{Motivation and previous work}

Xilinx FPGAs provide access to the configuration memory through an internal port known as Internal Configuration Access Port (ICAP). This port has a parallel communication interface that allows both sending commands and reading/writing configuration data.

In order to facilitate the usage of this port, Xilinx provides an IP core, the Hardware ICAP (HWICAP), which provides an interface between the microprocessor and the ICAP.

The main problem with this core is that it is accessed through the system bus via registers, providing no burst access option. This greatly reduces the speed of the system, as single transactions through this bus take approximately 10 clock cycles to complete, whereas the ICAP can work at one word per clock cycle. $^{1}$

Additionally, the ICAP has a problem: it can only address whole frames, which means that it cannot directly reconfigure regions smaller than one clock region in height. This is typically not a problem for the intended use of the

\footnotetext{
${ }^{1}$ The Zynq family of all-programmable SoCs provides a port accessible by the processing system, the PCAP, which can perform direct memory access at a speed of one word per clock cycle, so it is not necessary to use the ICAP at all on these devices unless hardware-managed reconfiguration is desired.
} 
partial reconfiguration capabilities of Xilinx FPGAs, which is the coarse grain reconfiguration of big reconfigurable regions. However, EHW typically has very small PEs spanning much less than a clock region in height, and thus needs to perform fine grain reconfiguration. Therefore, in order to achieve this reconfiguration granularity with the HWICAP, the configuration of a whole column should be generated by combining the bitstream of the new element with the preexisting configuration of said column. Doing this combination in software would further slow down the reconfiguration process, and therefore is not a satisfactory solution.

In order to overcome this problem, previous work [Otero'10] presents an alternative hardware-accelerated reconfiguration engine. This engine is based on the one provided by Xilinx, but is able to perform direct memory access in order to load configuration data from memory using burst transactions, thus being much more efficient than the one provided by Xilinx.

Additionally, this engine is able to perform a readback of the current configuration data on the FPGA, save it on an internal memory, and write it back replacing a specific segment with data fetched from the external memory, thus being able to perform fine grain (sub-frame) DPR directly, without the intervention of software.

The nominal frequency of the ICAP is $100 \mathrm{MHz}$; nevertheless previous work [Claus'10, Hansen'11] shows that it can be overclocked to much higher frequencies.

However, the aforementioned reconfiguration engine has some drawbacks:

- Although designed with the purpose of some reusability, changing the target FPGA involves modifying a VHDL package file, which hinders the extension of the system to other FPGA models.

- Although it does support hardware-accelerated fine grain DPR, it is not optimized for it, being oriented mainly to coarse grain DPR.

- It relies on external RAM memory. This is necessary for big bitstreams; however, for very small ones such as those used in fine grain reconfiguration it would be more efficient to use BRAM blocks.

- It cannot address specific frames within a column individually. This will be needed in $\$ 5.1$, where reconfiguration of whole CLB columns will be replaced by reconfiguration of a few frames.

- Its control is somewhat complicated, which is not a problem if the engine is to be controlled by a microprocessor, but limits the possibility of implementing the evolution fully in hardware [Lanza'13]. 
- Experiments show that this core does not operate correctly when the ICAP is overclocked if readback is involved.

The need to efficiently perform fine grain DPR as needed by EHW has motivated the development of a new reconfiguration engine.

\subsubsection{Description of the chosen solution}

In order to overcome the aforementioned problems, a new reconfiguration engine targeting fine grain reconfiguration has been originally developed. This engine follows a different approach: instead of reading the current configuration from the column containing the region to reconfigure and rewriting it with some changes, as done by [Otero'10], it regenerates the whole configuration directly. This implies knowing the current configuration of all the regions in the column, not only the one that needs to be reconfigured.

Original contribution 4-2 A reconfiguration methodology based on readback-free sub-frame DPR by on-the-fly combination of preexisting DPR cells. A similar approach is followed in [Dobai'14] for LUT reconfiguration; however, this methodology is applicable to fine-grain DPR applications in general.

An advantage of this approach is that it halves the reconfiguration time since it removes the readback stage. Additionally, it allows configuring multiple elements at once when they are in the same column, thus potentially reducing the reconfiguration time.

Partial bitstreams used by this engine are stored on a BRAM. This is necessary so that random memory positions can be efficiently accessed, which is needed in order to compose the whole column to be reconfigured; using the external SDRAM efficiently would require performing burst accesses, which have an overhead which is proportionally larger the shorter the bursts are. Given the small size of the PEs, not much memory is needed to store the whole library of PE bitstreams. ${ }^{2}$

Original contribution 4-3 The replacement of external SDRAM by an internal BRAM on the reconfiguration engine, allowing easily interleaving PBSs to compose the global one without the overhead caused by jumping addresses in SDRAM.

\footnotetext{
${ }^{2}$ In the case of PEs with a size of $5 \times 2$ CLBs, the whole library would fit in just $45 \mathrm{~KB}$, which is a feasible size for a BRAM-based memory.
} 


\subsubsection{Implementation}

The engine is designed as a core VHDL module containing an ICAP instance, control ports, and a state machine to lead the configuration. This core module does not contain a BRAM memory nor configuration registers; those shall be implemented by an external wrapper file that communicates this core with the microprocessor bus.

The module reconfigures an area one frame in height, ${ }^{3}$ divided into multiple cells of arbitrary height, with each cell corresponding to one reconfigurable region.

\section{Control interface}

The module is controlled via the input/output port interface depicted in Figure 4-1.

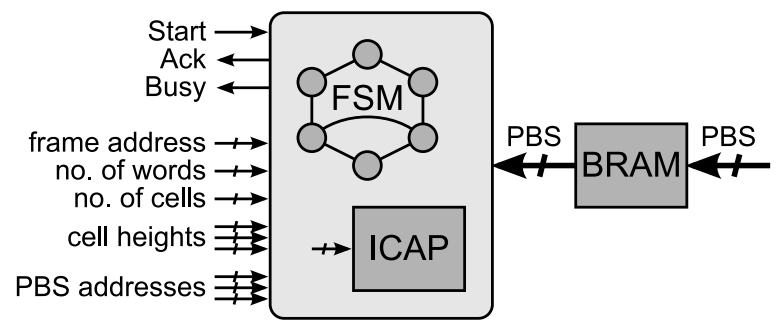

Figure 4-1: Signal interface of the generic reconfiguration module

The parameters for a specific reconfiguration operation (initial frame address, number and size of the individual cells, addresses in memory for the partial bitstreams of each cell, and size in words of the reconfigured area) are set by writing values on dedicated input ports. These values will be registered at the beginning of the operation. Normally, implementations will use fixed values for the number of cells and height of each cell, so these inputs will typically be tied to fixed values.

The reconfiguration process is triggered by a Start input signal. The module acknowledges this trigger with an Ack output, indicating that the configuration parameters have been registered and thus the external logic may change them in order to prepare for the next request. Finally, a Busy output is used to indicate whether or not the reconfiguration operation has finished.

\footnotetext{
${ }^{3}$ Reconfiguring regions spanning multiple clock regions vertically is not directly supported and would require doing so in multiple stages; this is out of the scope of this fine-grain reconfiguration engine.
} 
Additionally, the module has an interface to access an external BRAM memory where the PBSs will be stored.

As it was said, previous work [Claus'10, Hansen'11] shows that, although the nominal frequency of the ICAP is $100 \mathrm{MHz}$, it is possible to overclock it to obtain shorter reconfiguration times. In order to allow the use of different clocks for the reconfiguration module and the rest of the system, the Start and Ack ports use a two-phase handshake: they are asserted by toggling their value rather than relying on a one-cycle pulse (Figure 4-2).

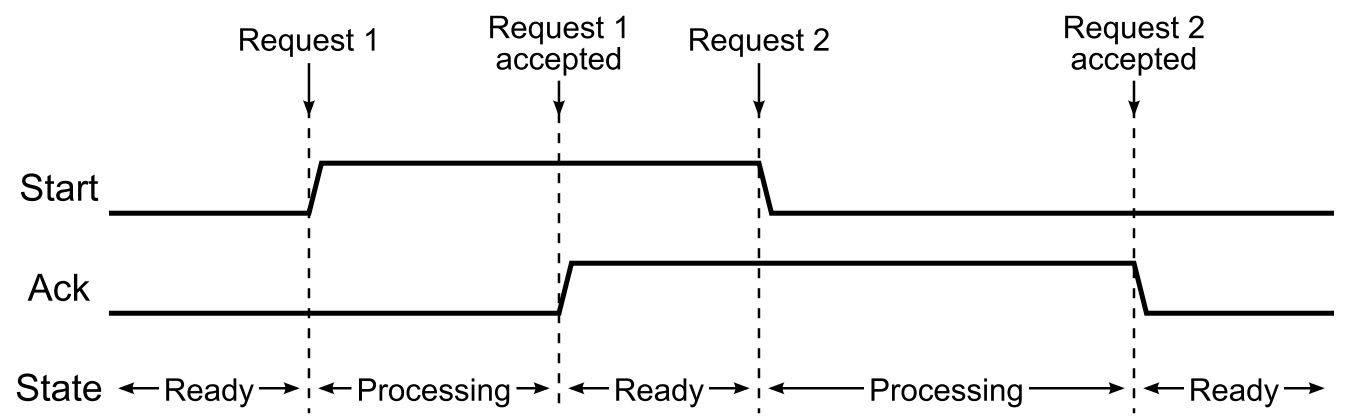

Figure 4-2: Two-phase handshake. The module is ready to receive new requests whenever Ack is equal to Start.

By having separate Ack and Busy signals, the microprocessor can start preparing a new request as soon as the reconfiguration module has registered the current one, without having to wait for it to be completed.

\section{Reconfiguration sequence}

The reconfiguration process is performed by a state machine that performs the following steps:

1. Wait for Start to be asserted.

2. Set the Busy signal, establish communication with the ICAP, and send a sequence of configuration commands (header) to initialize the configuration. ${ }^{4}$

3. Register the configuration parameters from the module input (frame address, number of words, cell number and size, and cell configurations) and assert the Ack signal.

\footnotetext{
${ }^{4}$ Header and tail are identical for all Virtex-5 FPGAs, with the exception of the IDCODE, which is a unique identifier for each FPGA model. The module is parameterized so that this IDCODE can be changed, making the module suitable for all Virtex-5 FPGAs without requiring modifications on the HDL code.
} 
4. Setup ICAP to write on the desired frame address.

5. Write the configuration information frame by frame by accessing the external BRAM in an interleaved manner.

6. If Start has been asserted again during steps 4 and 5, repeat from step 3; otherwise continue.

7. Write another command sequence (tail) to finish the communication with the ICAP, clear the Busy signal, and go back to step 1.

This way, if a new request is issued before the configuration has finished, the module can skip steps 2 and 7, performing all the configurations on a single connection with the ICAP.

The commands needed to reconfigure the FPGA (steps 2, 4, and 7) are detailed in [Xilinx-UG191].

\section{Implementation as an IP core}

The module described here is a generic one suitable for a high number of designs. However, design of digital systems typically requires modules to be implemented as processor peripherals in the form of IP cores. Thus, the reconfiguration engine will be wrapped on an IP core along with a block RAM to host the partial bitstreams and some configuration registers that will be accessible by the processor through a bus.

Given the simplicity of the described module, it is easy to generate custom IP cores that will suit a specific need. For example, the frame address can be passed from a processor-accessible register, letting the software running on the processor access arbitrary addresses, or it can be tabulated for a simple control if the PE positions are going to be fixed.

An implementation of this reconfiguration engine tailored for an SA will be described in $\$ 5.1 .5$, and a generalized version that may be used for other finegrain applications will be proposed in $\S 7.2 .2$. 


\subsection{PARTIAL BITSTREAM EXTRACTION: METHODOLOGY AND TOOLS}

Designs based on DPR need to overwrite part of the configuration data stored in the FPGA memory with new data corresponding to the circuit to be configured using a partial bitstream. The tools provided by the FPGA vendor (Xilinx) contain utilities to obtain this partial bitstream in their partial reconfiguration (PR) flow. However, due to limitations on this PR flow such as the impossibility of performing relocation, other approaches need to be followed (as detailed in \$3.1.2). Among other things, this means that a replacement for the PR flow to obtain these partial bitstreams from a design is necessary.

This section describes the advances made into the generalization of a tool to perform this task.

Original contribution 4-4 A PBS analysis and extraction tool, along with some derived applications, which are useful for current and future projects where DPR is involved.

\subsubsection{Motivation}

The reconfiguration engine described in $\$ 4.1$ is able to generate a series of commands for initiating and finishing the configuration. However, the configuration data that is written to the FPGA, i.e., the actual information of the digital circuit being configured, is obtained from an internal memory to which it must be previously written.

This information needs to be obtained separately. Typically, this involves creating a project of a digital system containing one of the circuits to be used in the reconfigurable design on a predefined area, and then using a tool to extract the configuration information of said circuit. This information will be transferred to the reconfiguration engine at start up so that it can be used at run time.

As it has been said, the partial reconfiguration flow provided by the FPGA vendor does not fulfill the needs of the current project due to the lack of features such as relocation. Therefore, design methodologies related to this feature will require the use of a custom extraction tool.

In order to facilitate the development of DPR-based systems, the tool to perform the extraction of a partial bitstream should be designed in such a way 
that it interferes as least as possible with the system development, and avoid being limited to a specific FPGA model in order to simplify future porting to other FPGAs.

In previous work, this task was carried out by a very simple $C$ program, which read a . bit file generated with Xilinx tools for a Virtex-5 LX110T FPGA and some parameters to determine the region limit, and generated a binary . pbs file with the raw binary content of said region—-the partial bitstream. ${ }^{5}$

However, this solution only covers a specific FPGA model, requiring to recompile a different version of the program if support for a different FPGA were desired. In order to make this approach generalizable to new FPGA models, it would be necessary to make the program extensible by the addition of modules without requiring to recompile it. Additionally, it would be desirable to provide the program with more interactivity and the ability to inspect the content of bitstreams, rather than having a fixed functionality. For these reasons, it was decided to create an extensible program that allowed easily adding specifications for new FPGAs in the form of text files.

The programming language chosen for this program is Python, which is an interpreted language with a rather simple syntax; this was seen as a potential advantage as it would allow adding support for new FPGAs in the form of Python scripts.

Rather than limiting the program to a single functionality, it was decided to design it in the form of a framework where all the bitstream handling functionality is put on a module, and the bitstream extraction program is just a regular Python program that makes use of this module. Thus, what started as a small project to make the existing bitstream extraction tool more extensible grew into a very complete framework, which not only allows the extraction of partial bitstreams but also the analysis of configuration sequences. This analysis has been helpful in the design of the reconfiguration engine described in $\S 4.1$, as well as the study of different FPGA models and families for future projects.

This framework has also been useful in other lines of development where manual extraction of partial bitstreams was required, as it was the case in early stages of development of the ARTICo ${ }^{3}$ architecture.

\footnotetext{
5 "Partial bitstream" can also be interpreted as a bitstream file with all the necessary information to reconfigure only a fragment of the FPGA, including all the configuration commands needed to initiate and terminate the reconfiguration. In this context, however, it only refers to the raw data that will be written to the configuration memory; the aforementioned commands will be generated independently.
} 


\subsubsection{Framework description}

The main functionality of this framework is written as a Bitstream class. This class has all the necessary utilities to load a regular . bit file, retrieve the configuration commands and some metadata, identify the beginning of the configuration data, and extract a fragment of said data so that it can be later saved as a file.

In order to make the tool easily extensible, the set of parameters describing each supported FPGA is supplied in the form of Python modules. For example, a Virtex-5 LX110T with FF1136 packing - the one present on XUPV5 boardswill load the module fpgas $/ 5 \mathrm{vlx} 110 \mathrm{tff} 1136$. py. Thus, adding support for additional FPGAs will only require creating the corresponding file in said directory.

These files need to be created manually for each FPGA model intended to be supported; nevertheless, they have a very simple syntax and only require concatenating identifiers for the different types of column, like:

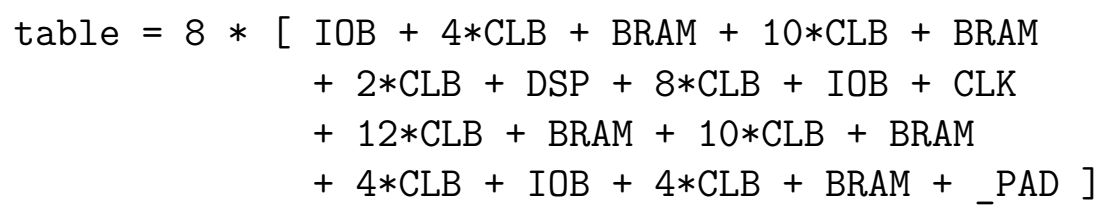

which describes the table of content of an FPGA with 8 identical rows with the described distribution of IOB, CLB, BRAM, DSP, and clock columns. ${ }^{6}$

These files also import a set of methods and parameters from a master FPGA definition file, which contains parameters common to all FPGAs in a family; for example, fpgas/5vlx110tff1136.py will import the master file fpgas/virtex5.py. This file defines properties such as word and frame size, and number of frames for different column types. Additionally, it defines a function that prints all the configuration commands in a readable form, allowing to display all these commands for a certain bitstream. This feature has been considerably helpful in the development and improvement of the reconfiguration engine described in $\$ 4.1$.

\footnotetext{
${ }^{6}$ The possibility of providing script files with a simple syntax as an extension mechanism has been one of the motivations for the choice of Python to develop this framework.
} 


\subsubsection{Derived applications}

Several applications have been developed using this framework. They are detailed below as example use cases.

\section{Command-line tool}

The first application that has been developed using this framework is a simple command-line tool for the direct extraction of partial bitstreams. This application can be run in an interactive manner (Figure 4-3), or it can be executed directly with a set of parameters from the command line, which allows integrating it within . bat or . sh scripts, permitting the automated extraction of multiple regions from several files.

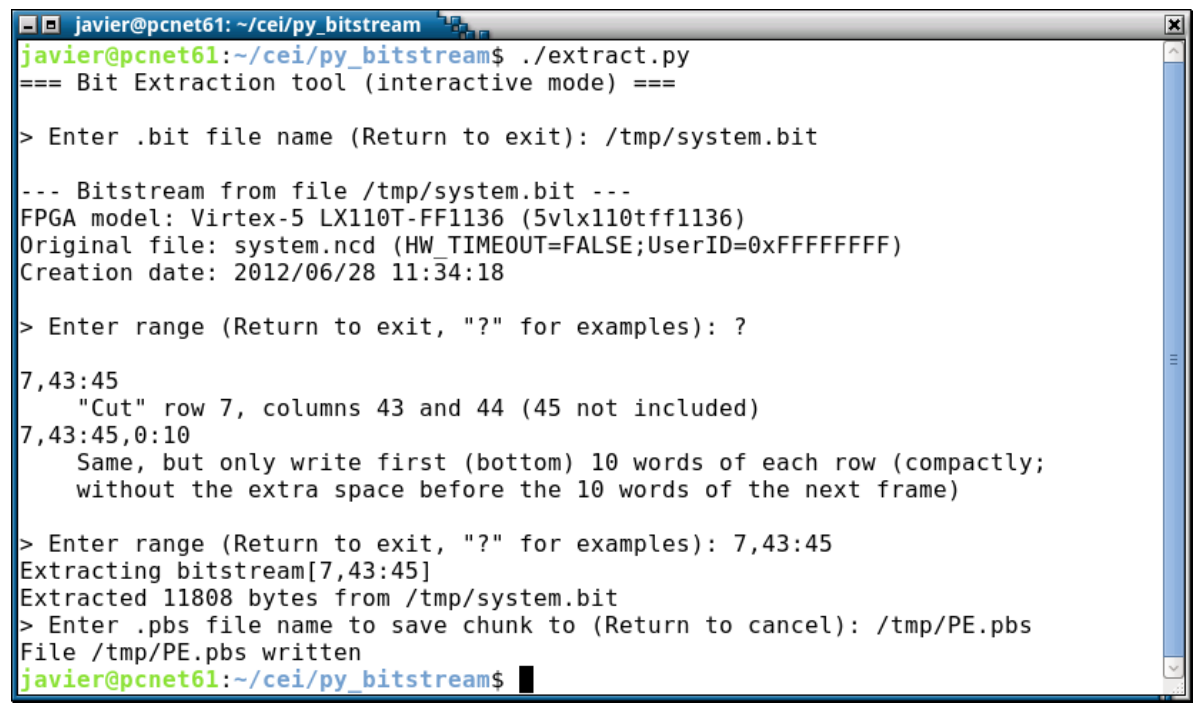

Figure 4-3: Example use of the command-line extraction tool in interactive mode

\section{Graphical application}

A problem with the aforementioned command-line tool was that it was not practical nor intuitive for a regular use. In order to facilitate the use of the framework to regular users, it was decided to develop a graphical user interface (GUI) that used the framework in a more user-friendly way. 
As can be seen in Figure 4-4, the interface is quite simple. It allows selecting a rectangular range with a granularity down to a single configuration word. ${ }^{7}$ Additionally, the application allows visualizing the content of the bitstream as plain text and dumping it to a file (Figure 4-5).

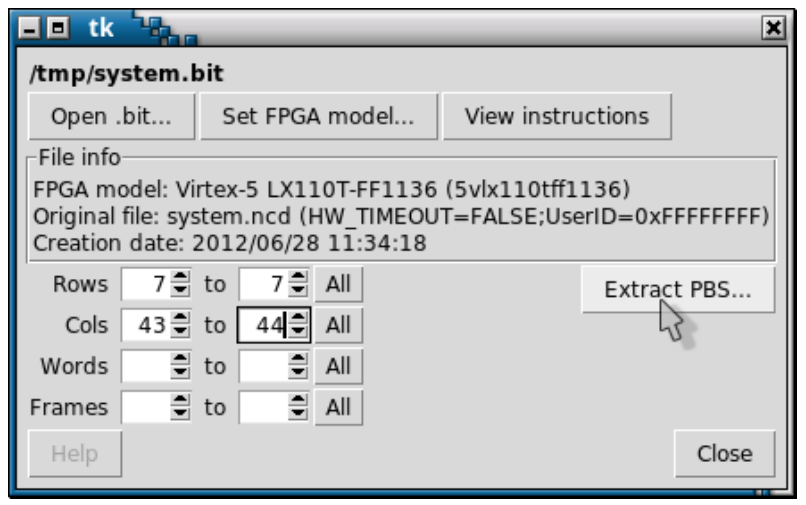

Figure 4-4: Partial bitstream extraction GUI

\section{Interactive console}

A useful application for the development of this framework was the interactive console (Figure 4-6). This tool is not as practical or user-friendly as the GUIbased application for extracting bitstreams, but is very useful for debugging and testing the different features implemented in this framework, and it gives the user a closer control of the framework rather than being limited to a fixed functionality.

\footnotetext{
${ }^{7}$ This feature is useful for extracting the bitstream corresponding to a single slice in order to reconfigure only logic elements, as will be required in $\$ 5$.1. Nevertheless, typical usage will use granularities of one row in height by one column in width.
} 


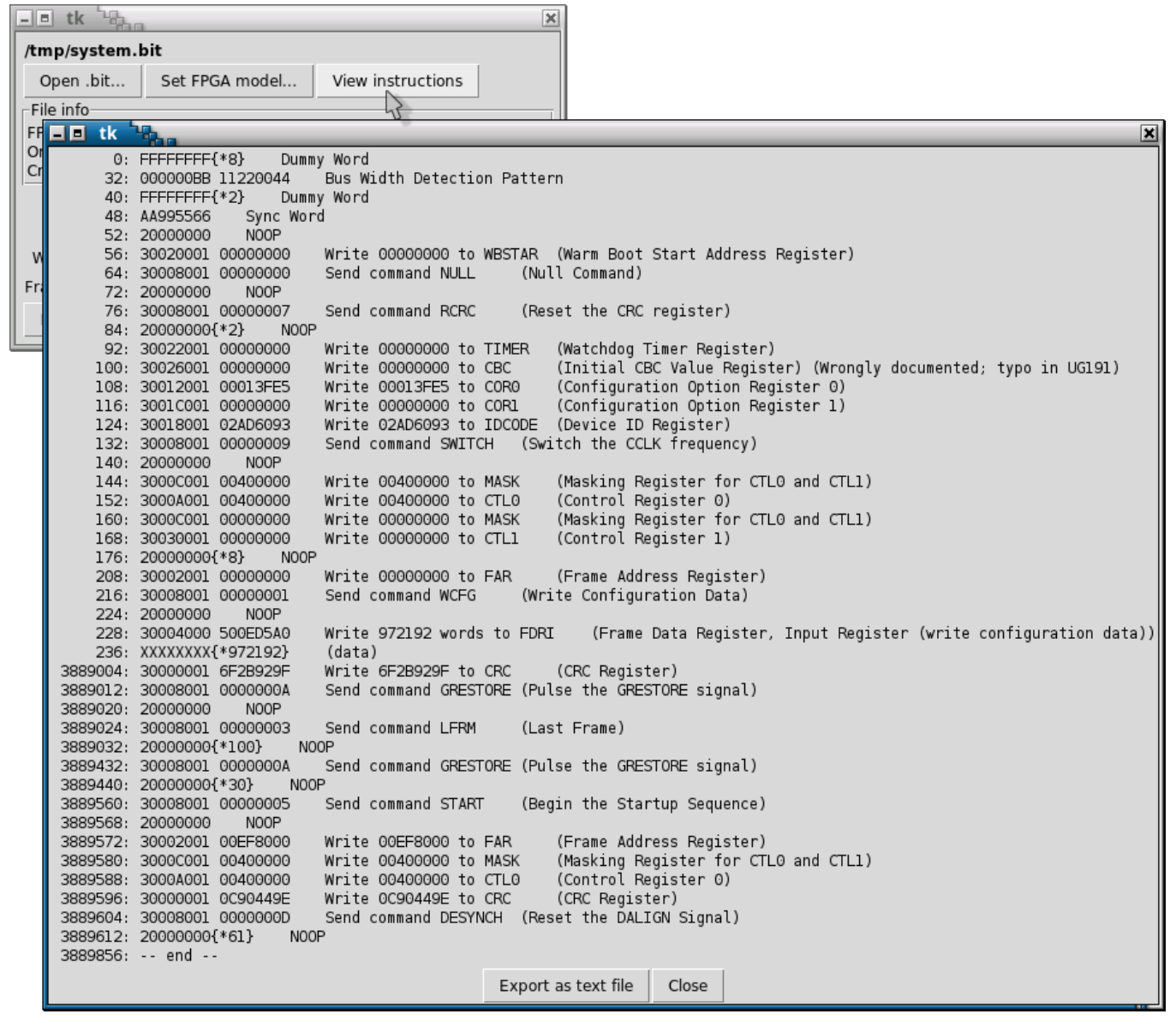

Figure 4-5: Visualization of the bitstream content 


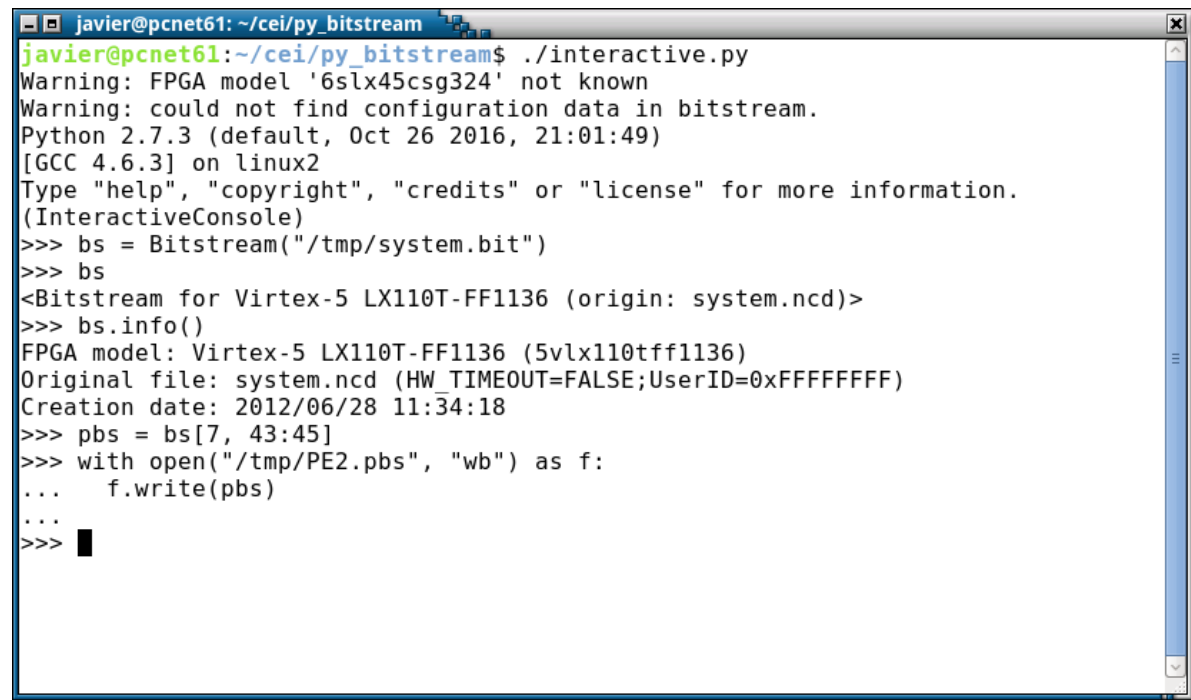

Figure 4-6: Example use of the interactive application. Unlike the command line tool in interactive mode, this tool gives the user more control over which actions to perform on a bitstream object. 


\section{ARCHITECTURE AND HARDWARE DESIGN METHODOLOGY}

This chapter analyzes different design choices for EHW and describes the different design methodologies adopted in order to obtain efficient EHW systems that can be easily integrated into projects.

First, a PE implementation using a LUT-based reconfiguration methodology is described in section 5.1. This approach results in a very compact and resource-efficient design, allowing for very large SAs. The compactness of this implementation and the removal of routing constraints results in an SA whose working frequency can reach $500 \mathrm{MHz}$ for a Virtex-5 FPGA, a performance level that is difficult to reach even for non-reconfigurable designs. Additionally, this LUT-based design approach has been deeply parameterized, resulting in a selfcontained system that can be easily integrated in a design by simply adjusting a few parameters.

This newfound resource efficiency and potentially large array size has opened the door to the exploration of the scalability of the proposed architecture. Following this premise, section 5.2 makes a comparative analysis between different topologies for EHW based on the new PE architecture. This section shows the differences in performance and resource usage between SA and CGP when used for EHW, studying the limitations of each solution and motivating the choice of an SA topology for the design. Additionally, scalability of both topologies is studied, showing that, although SA sizes of $4 \times 4$ PEs have been proven to yield satisfactory results, sizes of up to $10 \times 10$ could be considered as they provide a significant improvement on the results.

However, the aforementioned improvement in the maximum operating frequency of the SA does not directly impact the processing speed of the complete system, since logic elements surrounding the SA become a limiting factor. This has motivated a redesign of the whole filtering system, detailed in section 5.3, which favors a more modular design methodology with an optimized and simplified design, allowing different parts of the system to be optimized separately. Furthermore, the possibility of parallelizing the system in order to evaluate the fitness of multiple candidate solutions in parallel is introduced. 
This modularization has been exploited in section 5.4, which proposes a design methodology based on stream-oriented building blocks, permitting the design of more complex processing systems based on a dataflow paradigm.

The optimizations in the processing architecture and surrounding logic result in a system able to reach a throughput in the range of 400-500 megapixels per second. This results in a considerable speedup of the evolution stage, as well as a high performance once the SA has been evolved. Additionally, LUT-based reconfiguration simplifies the implementation and results in a great improvement in reconfiguration time, further increasing the evolution speed.

The modularization of the system makes its different parts easily reusable in new designs, thus promoting the proliferation of derived projects. Furthermore, future development could use the separate modules as building blocks for coarse grain reconfigurable systems, allowing to change parts of the design such as the fitness computing unit at run time.

\subsection{LUT-BASED RECONFIGURATION METHODOLOGY}

The reconfiguration methodology described so far involves configuring relatively large areas of the FPGA containing both logic and interconnections.

This method has the advantage of being very flexible in the range of functions that each PE can implement. However, it has some important limitations:

- Making a design suited to relocation-enabled DPR is very complex, as it requires the use of bus macros or of third party routing tools such as the DREAMS tool. This becomes an obstacle to the exploration of possible modifications and improvements on the system, such as larger array sizes or improvements in the control logic.

- Bus macros represent an extra resource usage and increase the delay, worsening the timing. Even if bus macros are not used, tools such as DREAMS will typically require the designer to oversize the circuit in order to give the routing process some freedom to choose a routing that meets the requirements. 
- It requires reconfiguring both logic and interconnections. The information related to interconnections represents more than $70 \%$ of the configuration bitstream on a Virtex-5 FPGA [Xilinx-UG191, § Frame Addressing]. A design that only required reconfiguring the logic would require a shorter reconfiguration time and therefore would accelerate the evolution. Additionally, it would reduce the required size of the PE library.

- The forced routing is probably not optimal. The manufacturer provides a routing algorithm that optimizes the routing for several factors, including timing and energy consumption.

Therefore, moving to a simpler design methodology is an important goal for the generalizability of the system, as it would allow exploring new applications and increase the range of applications of such a system.

As it was said in $\$ 2.2 .3$, other alternatives to DPR are the use of VRC (which involves a waste of resources and a lower performance) and using the SRLUT functionality of some LUTs to change their behavior (which is limited by the fact that only $25-30 \%$ of the LUTs have this functionality in Xilinx FPGAs).

An alternative to using the shift register functionality of LUTs to change their internal configuration is to use DPR. Unlike the current design model, this DPR would only be used on the logic elements of the FPGA and therefore would not require modifying the interconnections. This greatly simplifies the design of reconfigurable systems, since it only requires restricting the placement of logic elements on the design and not the routing: placement restrictions are very easy to specify and the manufacturer provides well documented tools for this [Xilinx-UG625].

Given the enormous benefit that LUT-based reconfiguration provides in terms of simplicity and resource efficiency, a PE design based entirely on reconfigurable LUTs has been developed. This has been possible with the collaboration of Ing. Roland Dobai, Ph.D. from the Brno University of Technology.

\subsubsection{Understanding Virtex-5 configurable logic blocks}

Xilinx FPGAs implement low-level logic elements such as registers, logic functions, adders, and distributed memory by using dedicated blocks known as configurable logic blocks (CLBs); the internal structure of these blocks is detailed in [Xilinx-UG190, ch. 5] for the Virtex-5 family (with other families such as the 7 Series following a very similar scheme). Each Virtex-5 CLB contains two slices, often represented on the bottom left and top right of the CLB (Figure 5-1). 


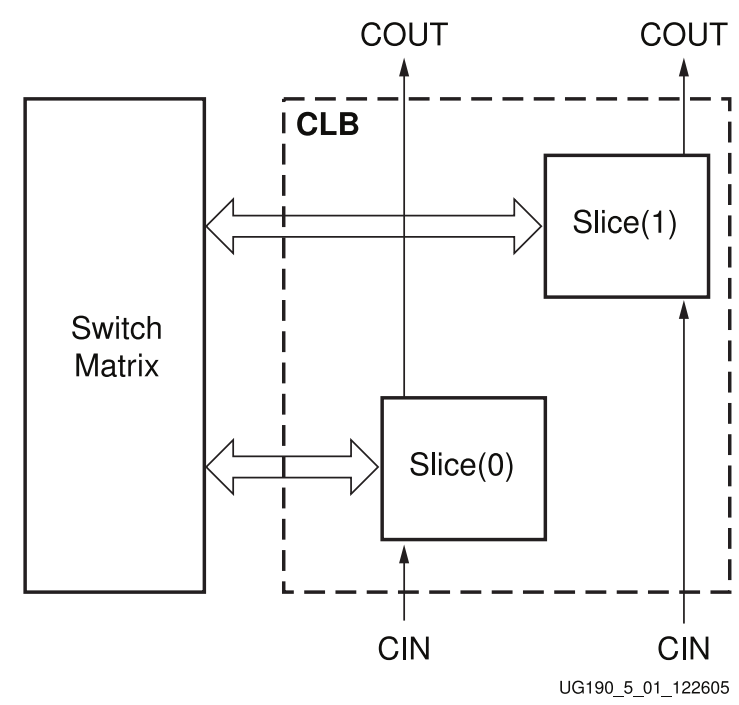

Figure 5-1: Arrangement of slices within a CLB. Image obtained from [Xilinx-UG190], (C) Xilinx, Inc.

Each of these slices contains four 6-input LUTs (labeled A, B, C, D), a 4-bit fast carry chain, three 1-bit multiplexers, and four FFs/latches, interconnected as shown in Figure 5-2. The LUTs can be used to implement arbitrary logic functions (such as a half-adder or a 4:1 multiplexer with a 2-bit select). The 4bit carry chain can be combined with the four LUTs to implement a cascadable 4-bit adder with fast carry propagation. The three 1-bit multiplexers can be used to extend the functionality of the four LUTs as a single 8-input LUT or a 16:1 multiplexer. Finally, the four FFs allow registering the slice output.

There are two types of slice in Virtex-5. The one depicted in Figure 5-2 is a SLICEL. Virtex-5 FPGAs also feature another type of slice known as SLICEM, which is similar to a SLICEL except that the LUTs have RAM capabilities, allowing them to work as distributed RAM or shift registers. The functionality of a SLICEM is a superset of that of a SLICEL.

The 6-input LUT is implemented as two 5-input LUTs as Figure 5-3 shows [Xilinx-UG621, §LUT6_2]. This allows using it as a 6-input 1-output LUT (by ignoring the $\mathrm{O} 5$ output) or as a 5-input 2-output LUT (by tying A6 to 1). Each of the 5 -input LUTs is implemented in such a way that lower inputs (A1, A2...) are slower than higher inputs (A5, A4...), so it is advisable to use the latter for 


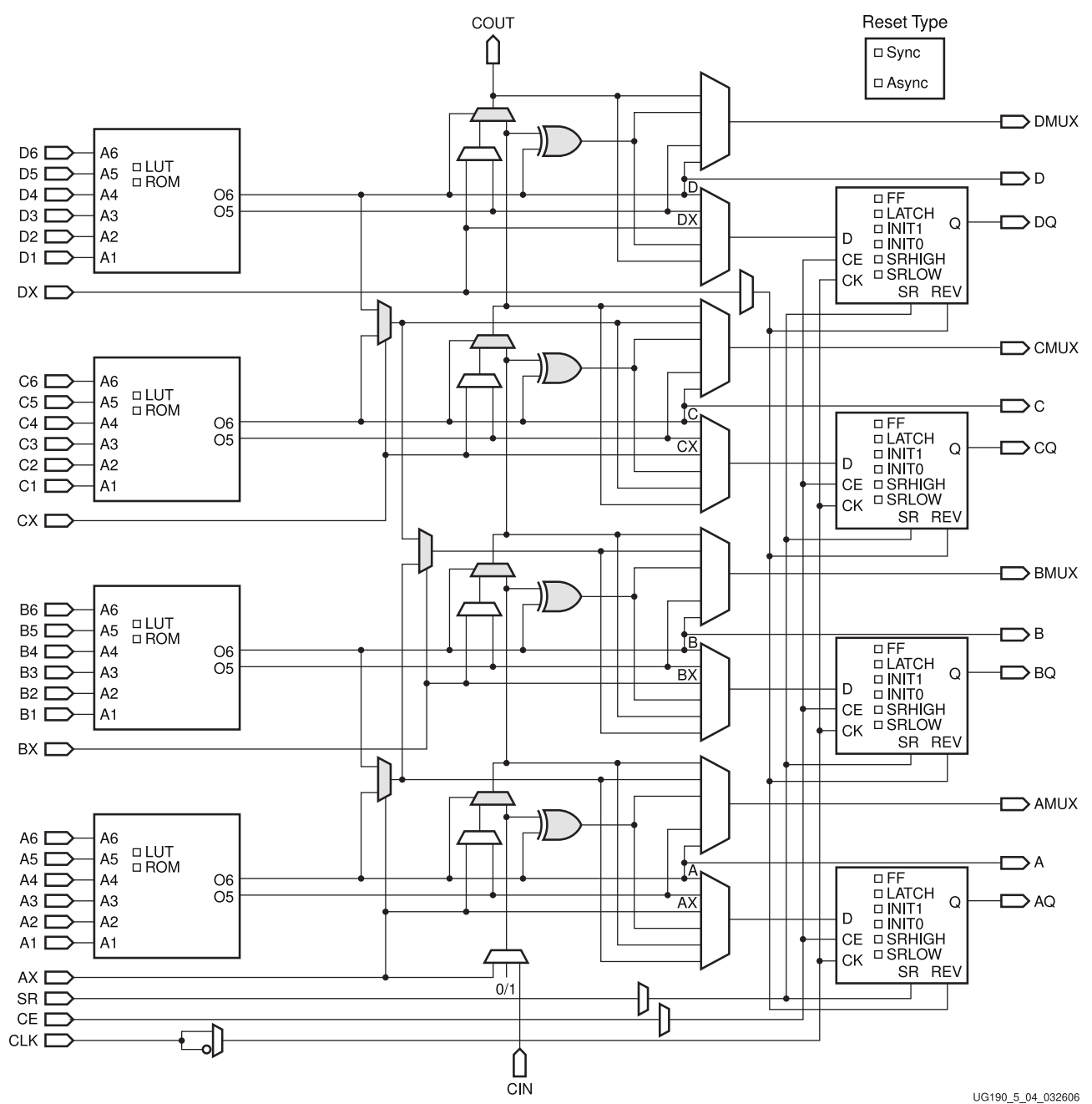

Figure 5-2: Structure of a Virtex-5 SLICEL. Image obtained from [Xilinx-UG190], (c) Xilinx, Inc.

timing-critical signals when designing with LUTs [Xilinx-UG904, $₫$ Critical Pin Optimization]. ${ }^{1}$

\footnotetext{
${ }^{1}$ [Xilinx-UG904] applies to FPGAs in the 7 Series and not to the Virtex-5. Nevertheless, some tests performed using FPGA Editor have shown a similar behavior for a Virtex-5 FPGA: nets routed from a FF to the Al pin of a LUT in the same slice have a delay approximately $0.5 \mathrm{~ns}$ longer than nets routed from the same FF to the A6 pin of the same LUT.
} 


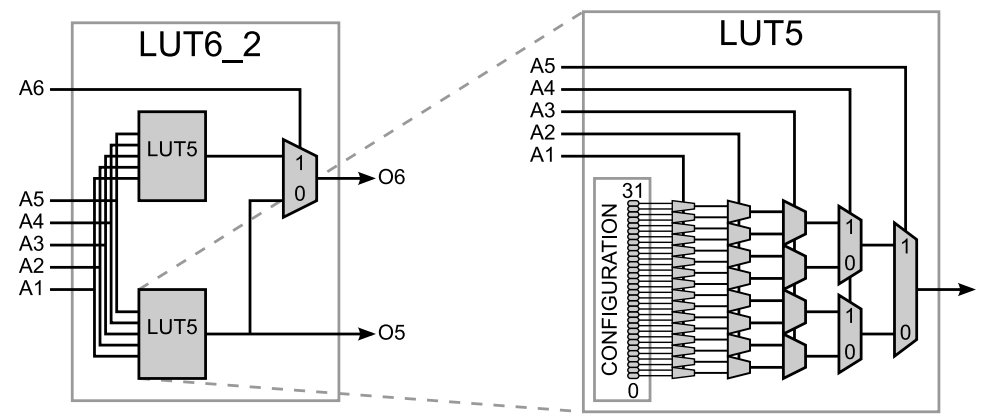

Figure 5-3: Left: Virtex-5 implementation of a 6-input LUT as two 5-input LUTs, which also allows its use as a 5-input 2-output LUT by tying A6 to 1 (this is documented in [Xilinx-UG621] as a LUT6_2 primitive). Right: possible implementation of each of the 5-input LUTs that illustrates their behavior (and would explain why input A1 is slower than A5). This scheme is speculative and may not reflect the actual implementation within the FPGA.

\section{Distribution of LUT content in the configuration memory}

In order to be able to change the configuration of a LUT, it is important to understand how this information is stored within the bitstream.

As explained in [Xilinx-UG191, § Frame Addressing], a CLB column—which contains 20 CLBs-is composed by 36 frames. Frames $0-25$ are used to encode the switch box interconnection configuration, leaving frames 26-35 for the CLB configuration. Each two words of each frame correspond to a CLB, with the first two words corresponding to the bottommost CLB, and the extra word in the middle of the frame is used for clock and error correction purposes.

The documentation does not specify the format in which the LUT content is encoded in these 10 frames. However, [Shayani'13, appx. A] shows this distribution in detail. This information is replicated in tables 5-1 and 5-2.

The conclusions obtained from these tables are:

- The LUT configuration is contained in frames 32-35 for the bottom slice, and 26-29 for the top slice (Table 5-1). Frames 30-31 likely contain other slice configuration information (FF behavior, reset synchronicity, FF input...).

- Each LUT spans 4 frames and uses 16 bits of each frame, which is consistent with the vertical word arrangement documented in [Xilinx-UG191, fig. 6-9]. 


\begin{tabular}{|c|c|c|c|c|c|c|c|c|}
\hline & \multicolumn{4}{|c|}{ Word 0} & \multicolumn{4}{|c|}{ Word 1} \\
\hline & Byte 0 & Byte 1 & Byte 2 & Byte 3 & Byte 0 & Byte 1 & Byte 2 & Byte 3 \\
\hline & $31 \ldots 24$ & $23 \ldots 16$ & $15 \ldots 8$ & $7 \ldots 0$ & $31 \ldots 24$ & $23 \ldots 16$ & $15 \ldots 8$ & $7 \ldots 0$ \\
\hline \multicolumn{9}{|l|}{ Frame 0} \\
\hline \multicolumn{9}{|l|}{$\ldots$} \\
\hline \multicolumn{9}{|l|}{ Frame 25 } \\
\hline \multicolumn{9}{|l|}{ Frame 26} \\
\hline \multicolumn{9}{|l|}{ Frame 27 } \\
\hline Frame 28 & \multirow{2}{*}{\multicolumn{2}{|c|}{ top slice }} & \multirow{2}{*}{\multicolumn{2}{|c|}{ top slice }} & \multirow{2}{*}{\multicolumn{2}{|c|}{ top slice }} & \multirow{2}{*}{\multicolumn{2}{|c|}{ top slice }} \\
\hline Frame 29 & & & & & & & & \\
\hline \multicolumn{9}{|l|}{ Frame 30} \\
\hline \multicolumn{9}{|l|}{ Frame 31 } \\
\hline \multicolumn{9}{|l|}{ Frame 32} \\
\hline \multicolumn{9}{|l|}{ Frame 33} \\
\hline Frame 34 & \multirow{2}{*}{\multicolumn{2}{|c|}{ bottom slice }} & \multirow{2}{*}{\multicolumn{2}{|c|}{ bottom slice }} & \multirow{2}{*}{\multicolumn{2}{|c|}{ bottom slice }} & \multirow{2}{*}{\multicolumn{2}{|c|}{ bottom slice }} \\
\hline Frame 35 & & & & & & & & \\
\hline
\end{tabular}

Table 5-1: Distribution of the content of the 4 LUTs of each slice across the configuration frames.

-SLICEL-

\begin{tabular}{|c|c||cccccccc|cccccccc|}
\hline \multicolumn{1}{|c||}{ Slice } & \multicolumn{8}{|c|}{ Byte 0/2 } & \multicolumn{7}{|c|}{ Byte $1 / 3$} \\
\hline bottom & top & 15 & 14 & 13 & 12 & 11 & 10 & 9 & 8 & 7 & 6 & 5 & 4 & 3 & 2 & 1 & 0 \\
\hline \hline Frame 32 & Frame 26 & 2 & 7 & 10 & 15 & 18 & 23 & 26 & 31 & 34 & 39 & 42 & 47 & 50 & 55 & 58 & 63 \\
\hline Frame 33 & Frame 27 & 3 & 6 & 11 & 14 & 19 & 22 & 27 & 30 & 35 & 38 & 43 & 46 & 51 & 54 & 59 & 62 \\
\hline Frame 34 & Frame 28 & 1 & 4 & 9 & 12 & 17 & 20 & 25 & 28 & 33 & 36 & 41 & 44 & 49 & 52 & 57 & 60 \\
\hline Frame 35 & Frame 29 & 0 & 5 & 8 & 13 & 16 & 21 & 24 & 29 & 32 & 37 & 40 & 45 & 48 & 53 & 56 & 61 \\
\hline
\end{tabular}

-SLICEM-

\begin{tabular}{|c|c||cccccccc|cccccccc|}
\hline \multicolumn{1}{|c||}{ Slice } & \multicolumn{1}{|c|}{ Byte 0/2 } & \multicolumn{1}{|c|}{ Byte 1/3 } \\
\hline bottom & top & 15 & 14 & 13 & 12 & 11 & 10 & 9 & 8 & 7 & 6 & 5 & 4 & 3 & 2 & 1 & 0 \\
\hline \hline Frame 32 & - & 1 & 4 & 9 & 12 & 17 & 20 & 25 & 28 & 33 & 36 & 41 & 44 & 49 & 52 & 57 & 60 \\
\hline Frame 33 & - & 0 & 5 & 8 & 13 & 16 & 21 & 24 & 29 & 32 & 37 & 40 & 45 & 48 & 53 & 56 & 61 \\
\hline Frame 34 & - & 3 & 6 & 11 & 14 & 19 & 22 & 27 & 30 & 35 & 38 & 43 & 46 & 51 & 54 & 59 & 62 \\
\hline Frame 35 & - & 2 & 7 & 10 & 15 & 18 & 23 & 26 & 31 & 34 & 39 & 42 & 47 & 50 & 55 & 58 & 63 \\
\hline
\end{tabular}

Table 5-2: Position of LUT entries in the bitstream on a Virtex-5 FPGA, for both SLICEL and SLICEM. Top slices are always of type SLICEL; bottom slices are SLICEL on some CLB columns and SLICEM on others. The LUT entry for a certain input is represented as a number whose binary representation is A6...A1 (e.g. 32 for A6=1, A5 .. 1=0). 
- Each LUT entry (i.e., the output for each possible input) is encoded as a single bit on the bitstream, using a 0 for an output of 0 and a 1 for an output of 1 .

- The LUT entries do not follow a natural order but a rather convoluted one, as can be seen in Table 5-2.

- The order is different for SLICEL and SLICEM. More specifically, the order in which the four frames are arranged is different, but the order within each frame is the same.

The fact that SLICEL and SLICEM have a different order complicates things, as it would require having different partial bitstreams for both slice types. A solution to this is to tie the $\mathbf{A 2}$ input to $\mathbf{0}$. This reduces the number of LUT entries that are used so that they are all contained in two consecutive frames (Table 5-3), and additionally the order of these two frames is the same for SLICEL and SLICEM, allowing these two slice types to be configured using the same partial bitstream, changing only the frame address where the configuration starts.

The number of frames to reconfigure can be further reduced to one by tying A1 and A3 to 0 as well (or, if an extra input is needed, it is possible to obtain the same result by connecting both $\mathrm{A} 1$ and $\mathrm{A} 3$ to the same input), which can be useful for reducing the reconfiguration time and the PBS size.

\section{Considerations for moving to 7 Series Xilinx FPGAs}

As it was mentioned in $\$ 3.5 .5$, Virtex-5 FPGAs are not supported by newer Xilinx tools and may become obsolete in a near future. In order to avoid getting locked in an obsolete technology, the same considerations for LUT reconfiguration regarding the bitstream content have been made for FPGAs in the 7 Series, such as Artix, Kintex, and the Zynq all-programmable SoC.

These families of FPGAs are very similar to Virtex-5, with the main differences being the presence of twice as many FFs per slice and a larger CLB column size (of 50 CLBs rather than 20, resulting in larger frames [Xilinx-UG470]). The distribution of LUTs within the configuration frames is the same, so Table 5-1 still applies; however, the distribution of the LUT content changes. Similarly to Virtex-5, 7-Series FPGAs have a different frame order for SLICEL and SLICEM LUTs. This problem has the same solution as before, but in this case tying A4 to 0. 
-SLICEL-

\begin{tabular}{|c|c|c|c|c|c|c|c|c|c|c|c|c|c|c|c|}
\hline \multicolumn{2}{|c|}{ Slice } & \multicolumn{6}{|c|}{ Byte $0 / 2$} & \multicolumn{8}{|c|}{ Byte $1 / 3$} \\
\hline bottom & top & 15 & 14 & 13 & $\begin{array}{lllll}3 & 12 & 11 & 10\end{array}$ & 9 & 8 & 7 & 6 & 5 & 4 & 3 & 2 & 1 & 0 \\
\hline Frame 32 & Frame 26 & & & & & & & & & & & & & & \\
\hline Frame 33 & Frame 27 & & & & & & & & & & & & & & \\
\hline Frame 34 & Frame 28 & 1 & 4 & 9 & $\begin{array}{lll}12 & 17 & 20\end{array}$ & 25 & 28 & 33 & 36 & 41 & 44 & 49 & 52 & 57 & 60 \\
\hline Frame 35 & Frame 29 & 0 & 5 & 8 & $\begin{array}{lll}13 & 16 & 21\end{array}$ & 24 & 29 & 32 & 37 & 40 & 45 & 48 & 53 & 56 & 61 \\
\hline
\end{tabular}

-SLICEM-

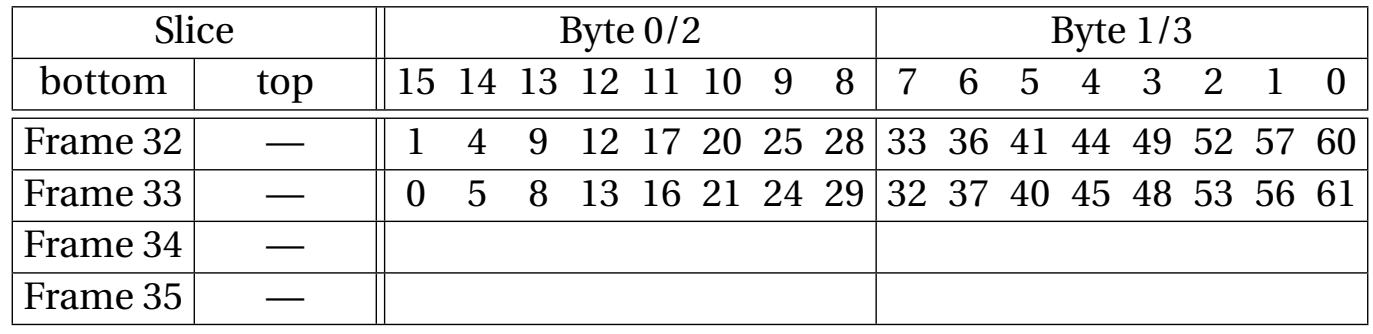

Table 5-3: LUT entry usage with A2 tied to 0 . Notice how 2 and 3 (000010 and 000011) are skipped, as well as any other number with the second bit from the right set to 1 . If $\mathrm{Al}$ and $\mathrm{A} 3$ are also connected to 0 or to the same input, only frame 35/29/33 will be used.

\subsubsection{Development of LUT-based PEs}

In order to move to a LUT reconfiguration methodology, the architecture of the PEs has to be redesigned so that it consists of a common structure based on LUTs whose functionality can be entirely changed by modifying the content of these LUTs. This has been possible through a collaborative effort with Prof. Roland Dobai from Brno University of Technology, who contributed the original idea of the LUT-based PE architecture, which has later been modified in order to improve its performance and reduce the reconfiguration time.

\section{Structural description}

In order to implement a PE structure that can be reconfigured by only reconfiguring the content of a few LUTs, the high level behavioral PE design that used arithmetic functions has been replaced by a lower level structural design which instantiates and interconnects FPGA-specific components such as LUTs, FFs, and carry chains directly. Each PE consists of 8 "levels" to process each of the 8 bits of the inputs and output, which makes the design easily generalizable to $n$ bits. 
This design is divided into two stages (Figure 5-4). Stage 1 adds two $n$ bit values, returning an $n+1$-bit value (the most significant bit of this value is the carry out from the adder). These values can be either of the PE inputs, optionally bitwise negated (i.e. subtracted from $2^{n}-1$ ), or the constant values 0 or $2^{n}-1$.

Stage 2 selects either of two $n$-bit values depending on the value of the most significant bit of stage 1 (i.e. the carry out of the adder). These values can be either of the PE inputs, the result of stage 1 discarding the most significant bit (i.e. modulo $2^{n}$ ), or the result of stage 1 discarding the least significant bit (i.e. divided by 2 and rounded down), all of them optionally bitwise negated; or the constant values 0 or $2^{n}-1$.

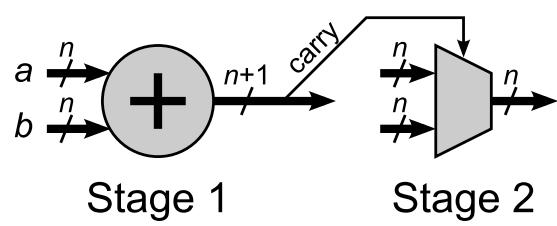

Figure 5-4: 2-stage structure of a PE. Inputs to both stages can be configured. The output of stage 1 includes the carry bit, and therefore has one bit more than each of the inputs. The carry bit is also used as the selector in stage 2 .

Stage 1 can be used as an adder/subtractor or as a comparator, ${ }^{2}$ and stage 2 can perform a conditional operation on the result (such as a selection or a saturation in case of overflow). This approach allows implementing all of the functions described in [Salvador'11a], as can be seen in Table 5-4-some of these implementations are not obvious and require some careful reasoning.

In addition to these functions, the generalization of the PEs as a twostage structure opens the possibility of introducing a wide range of new functions, such as new arithmetic functions or step functions that return either 0 or $2^{n}-1$ depending on the value of the inputs. The definition given here would theoretically allow 2100 combinations, ${ }^{3}$ although many of

\footnotetext{
${ }^{2}$ For example, a way to obtain the maximum between $N$ and $W$ inputs is to check the result of adding $N$ and negated $W$ and choose the output according to whether or not it overflows:

${ }^{3}$ Each of the two inputs to stage 1 can take one of 6 possible forms, which-considering that addition is commutative-gives 21 possible unordered combinations, and each of the two inputs to stage 2 can take one of 10 possible forms; therefore, the total number of combinations considering commutativity is $21 \times 10 \times 10=2100$.
} 


\begin{tabular}{cc|cc|cc} 
& FUNCTION & \multicolumn{2}{|c|}{ STAGE 1} & \multicolumn{2}{c}{ STAGE 2} \\
$\#$ & equation & $a$ & $b$ & $S<2^{n}$ & $S \geq 2^{n}$ \\
\hline 0 & $N+W \bmod 2^{n}$ & $N$ & $W$ & $S^{*}$ & $S^{*}$ \\
1 & $N+N \bmod 2^{n}$ & $N$ & $N$ & $S^{*}$ & $S^{*}$ \\
2 & $W+W \bmod 2^{n}$ & $W$ & $W$ & $S^{*}$ & $S^{*}$ \\
3 & $\min \left(N+W, 2^{n}-1\right)$ & $N$ & $W$ & $S^{*}$ & $\overline{0}$ \\
4 & $\min \left(N+N, 2^{n}-1\right)$ & $N$ & $N$ & $S^{*}$ & $\overline{0}$ \\
5 & $\min \left(W+W, 2^{n}-1\right)$ & $W$ & $W$ & $S^{*}$ & $\overline{0}$ \\
6 & $\left\lfloor\frac{N+W}{2}\right\rfloor$ & $N$ & $W$ & $S / 2$ & $S / 2$ \\
7 & $22^{n}-1$ & - & - & $\overline{0}$ & $\overline{0}$ \\
8 & $\left\lfloor\frac{N}{2}\right\rfloor$ & $N$ & 0 & $S / 2$ & - \\
9 & $\left\lfloor\frac{W}{2}\right\rfloor$ & $W$ & 0 & $S / 2$ & - \\
10 & $N$ & - & - & $N$ & $N$ \\
11 & $W$ & - & - & $W$ & $W$ \\
12 & $\max (N, W)$ & $N$ & $\bar{W}$ & $W$ & $N$ \\
13 & $\min (N, W)$ & $N$ & $\bar{W}$ & $N$ & $W$ \\
14 & $\max (N-W, 0)$ & $\bar{N}$ & $W$ & $\overline{S^{*}}$ & 0 \\
15 & $\max (W-N, 0)$ & $\bar{W}$ & $N$ & $\overline{S^{*}}$ & 0
\end{tabular}

Table 5-4: Two-stage implementation of the PE functions listed in Table 3-1. $S^{*}$ represents the output of stage 1 modulo $2^{n}, S / 2$ represents the output of stage 1 divided by 2 and rounded down, and $\bar{x}$ the bitwise negation of $x$.

these are redundant. ${ }^{4}$ In order to facilitate the addition of new functions, the development of a Matlab script that finds the combination that best approximates a user-supplied function will be discussed in $\$ 7.2 .4$.

It should be noted that this implementation also allows certain LUT configurations which would permit functions such as bitwise AND of the inputs and many other non-arithmetic operations. However, this possibility has not

\footnotetext{
${ }^{4}$ This number could be further reduced to 1800 , since $N+\bar{N}=W+\bar{W}=0+\overline{0}=2^{n}-1$ and therefore 2 of the 21 combinations in stage 1 are redundant, and also a stage 1 of $0+0$ does not provide any information that could not be generated on stage 2 directly. Additionally, certain stage 1 combinations such as those producing a fixed carry out will cause some stage 2 combinations to be redundant.
} 
been considered, following the assumption that arithmetic operations make more sense for digital signal processing applications.

\section{Detailed implementation}

The first implementation design of a LUT-based PE intended for our systolic array system was proposed by Prof. Roland Dobai from Brno University of Technology, as part of a collaborative project. The proposed structure is shown in Figure 5-5.

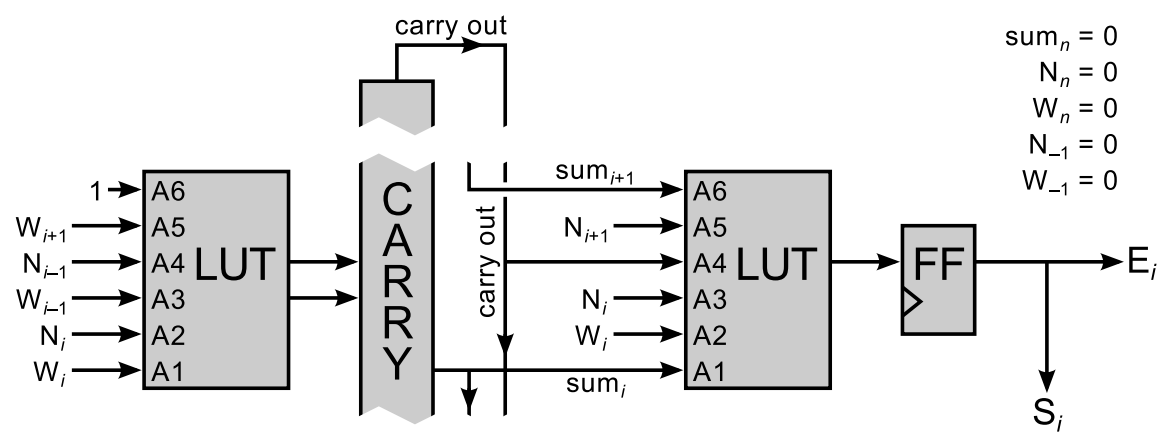

Figure 5-5: Fragment of a LUT-based PE, first version. Each PE is constituted by $n$ fragments like this, one per bit.

For a data size of $n=8$, this structure uses 16 LUTs, 8 FFs, and 2 cascaded 4-bit carry chains.

This configuration is very versatile and allows implementing a large number of functions. However, it presents some disadvantages:

- Using all six LUT inputs results in unavoidable inconsistencies between SLICEL and SLICEM, as explained in $\$$ 5.1.1. This could be solved by not using SLICEM or by having different versions of the partial bitstream for both.

- The output of stage 1 is extended with a 0 rather than with the carry bit, therefore computing a sum divided by 2 will involve an overflow. This can be easily solved by explicitly making the most significant LUT make its output equal to the carry bit, but this involves using different configurations for each of the LUTs, which makes the generation of partial bitstreams slightly more complicated. ${ }^{5}$

\footnotetext{
${ }^{5} \mathrm{An}$ advantage of having a homogeneous configuration for all LUTs is that the partial bitstream for a frame corresponding to two CLBs (8 LUTs) will consist of 4 identical words. This characteristic will be exploited in $\$ 7.2 .2$ to compress the PBS library to a quarter of its size.
} 
- Low LUT inputs are generally slower than high ones [Xilinx-UG904, $\S$ Critical Pin Optimization]; therefore feeding the output of stage 1 to the lowest input of stage 2 will cause a very bad performance.

- Many of the inputs are unnecessary for the current set of functions, although they allow implementing some extra functions such as $4 N, 4 \mathrm{~W}$, and $W / 4$ (but not $N / 4$ ). Reducing the number of inputs would reduce the amount of frames to be reconfigured, which would result in a faster reconfiguration (see $\$ 5.1 .1$ ).

In order to overcome these issues, the architecture was originally simplified as shown in Figure 5-6.

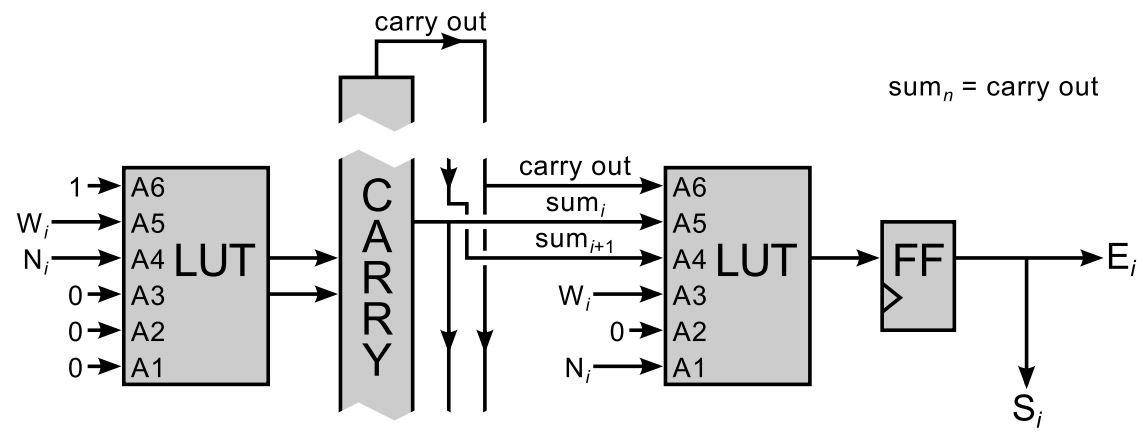

Figure 5-6: Fragment of a LUT-based PE, improved version. Each PE is constituted by $n$ fragments like this, one per bit.

This design removes unnecessary inputs, and rearranges the remaining ones in order to obtain a design as fast as possible. As it can be seen, the LUT on the first stage does not use the slow inputs A1-A3, and the one on the second stage uses them for the $N$ and $W$ inputs, which are less critical than the outputs of stage 1. This improves the design timing considerably, reducing the critical path by roughly $1 \mathrm{~ns}$, which results in an increase of the maximum operating frequency from 260 to $350 \mathrm{MHz}$.

Additionally, tying the unused inputs to 0 ensures that both SLICEL and SLICEM can be configured using the same partial bitstream, and reduce the number of frames to be reconfigured to 1 (for the first stage) and 2 (for the second stage).

Despite the simplifications, this PE structure is able to implement all of the required functions, using the configurations listed in Table 5-4. 
Original contribution 5-1 An optimized mapping of LUT inputs for the proposed two-stage LUT-based design, which considerably improves timing, reduces the number of frames to reconfigure, and ensures that the same PBS can be used for both SLICEL and SLICEM LUTS.

\subsubsection{Extending the LUT-based reconfiguration methodology to input and output elements}

As it has been explained, the proposed systolic array implementation has three parts: the array itself, consisting of multiple interconnected PEs; the input multiplexers, which allow selecting one of the 9 pixels of the $3 \times 3$ input sliding window; and the output multiplexer, which allows selecting the array output from the east PEs in order to avoid that a fault on a single PE can render the whole array useless.

Therefore, the reconfiguration process would use a hybrid approach: DPR would be used for PEs, whereas multiplexers would be configured by writing a value on a register. This not only implies requiring two different reconfiguration mechanisms, but also complicates the scalability of the design since larger SAs would require a larger number of registers. For the sake of consistency and simplicity, it would be preferable to rely exclusively on DPR to reconfigure the array.

In [Gallego' 13c], in order to achieve a scalable array, these multiplexers are replaced by hard-wired blocks (which will be referred to as selectors), which makes it possible to configure them by changing the block via DPR rather than writing to a register. Additionally, it introduces an alternative to simplify the interconnections on the input multiplexers: instead of generating a $3 \times 3$ window and routing the 9 values, it generates a $3 \times 1$ vertical window; the rest of pixels in the window are obtained by delaying the signal 0,1 , or 2 clock cycles in each selector (since the sliding window moves one pixel to the right per clock cycle, delaying the signal one clock cycle is equivalent to using the window value from one clock cycle before, i.e. one pixel to the left).

This approach has been combined with the idea of using LUT-based reconfigurable blocks to obtain LUT-based input and output selectors. Analogously to the LUT-based PEs, the new input selectors use a two-stage design (Figure 5-7), where the first stage selects one of three channels (window rows) and the second stage selects how much this signal is delayed in order to obtain different pixels in the row. This design can be easily extended to window sizes of $5 \times 5$ by adding two extra FFs per bit and adding LUT inputs A1 and A3 
on both stages. In order to improve performance, the output of the selector is registered.

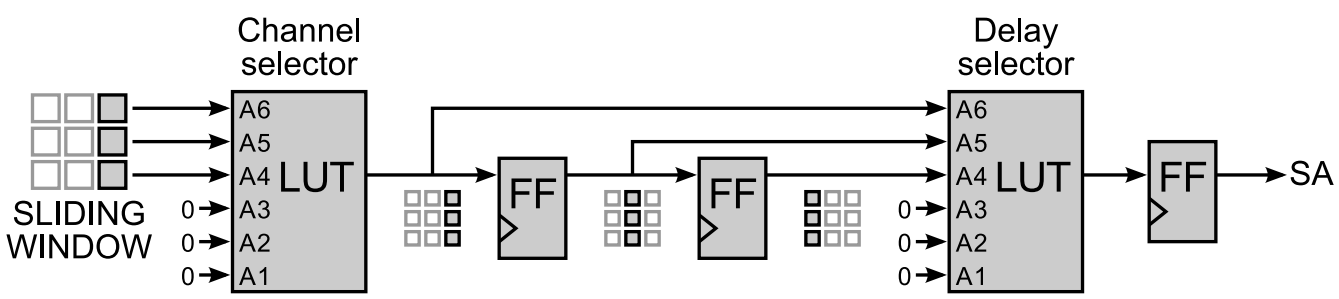

Figure 5-7: Fragment of a LUT-based input selector. Each input selector is constituted by $n$ fragments like this, one per bit.

In $\S 5.3 .3$ it will be discussed whether or not to add extra latency in order to have a consistently pipelined design. The system has been parameterized so that such extra latency can be added or not. Figure 5-8 shows how the input is registered in order to obtain said extra latency, which has the additional advantage of splitting long interconnections into shorter ones, potentially improving timing. Thanks to the two-stage approach, only three $n$-bit registers are needed per input instead of nine.

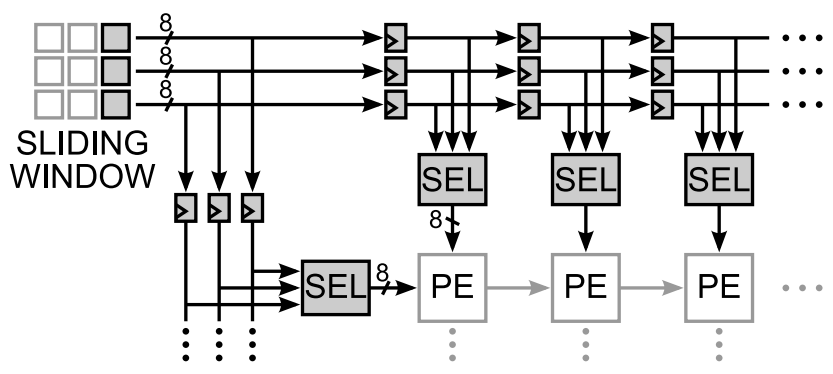

Figure 5-8: Pipelining of the input selectors

The output selector is not implemented as a single multiplexer, but as a series of cascaded selectors (Figure 5-9). This greatly simplifies the array scalability, but makes the use of intermediate registers necessary in order to obtain good timing. Each of the segments of the output selector (one per SA row) comprises a single stage of $n$ LUTs, with their A5 input connected to the output of the corresponding PE, the A6 input connected to the output of the segment above it, and the remaining inputs tied to 0 .

As shown in Figure 5-10, the additional registers added to the input and output selectors complete the pipelining of the array, making data propagate across the SA in separate wavefronts without interactions between different 


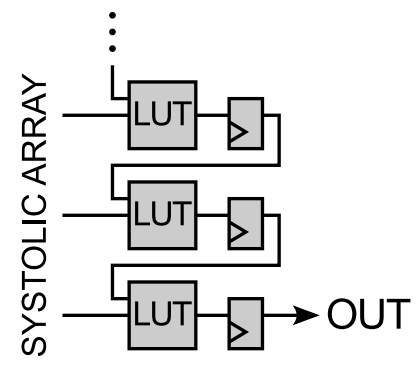

Figure 5-9: Diagram of the output selector (for 1 of the $n$ bits). The size of this selector depends on the height of the systolic array.

wavefronts. This makes the array latency totally predictable, depending exclusively on the array size and not its configuration.

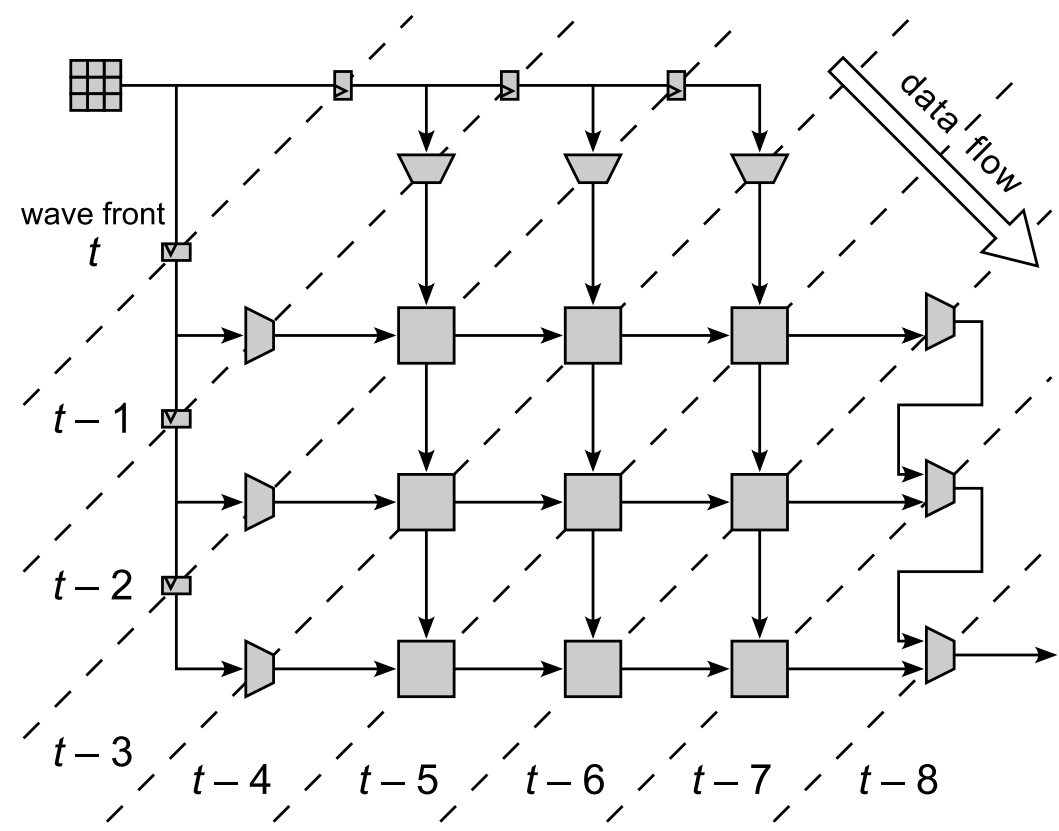

Figure 5-10: Pipelined data propagation through the SA in separate wavefronts

This design makes the input and output selectors behave as if they were extra PEs from a reconfiguration point of view, so PEs and selectors can be reconfigured simultaneously using the same DPR engine. The input selectors only need one frame to be reconfigured on each stage (unlike PEs which require two frames for stage 2); nevertheless configuring the rest of frames has no effect on their behavior. The output selector has one stage only, for which only one frame needs to be reconfigured. 
Original contribution 5-2 The implementation of input and output multiplexers as LUT-based selectors that can be configured using DPR, making the whole configuration process homogeneous.

It should be noted that this design is not oriented towards fault tolerance: a fault on the bottommost segment of the output selector breaks the whole array and cannot be circumvented. A solution to this could be to implement this selector using a fault tolerant strategy such as triple modular redundant chains [Lee'01]. The same applies to the chain of input selectors. However, this falls out of the scope of this thesis and should be considered as possible future work -in any case, fault tolerance on the input and output multiplexers was not being considered before.

\subsubsection{Placement constraints}

Unlike regular hardware design, partially reconfigurable design not only comprises the HDL specification but also a series of constraints to determine where the reconfigurable region should be and what layout it has. Typically, this involves specifying the placement of logic components and the routing of the interconnections between the reconfigurable region and the static system. Nevertheless, the current approach only requires reconfiguring the logic components while preserving the interconnections, so only placement constraints need to be applied.

Specifically, two types of placement constraints have to be applied:

- Exact positions of LUTs so that they are arranged orderly and in columns, in order to facilitate the reconfiguration.

- Positions of the FPGA that should not contain any logic, so that reconfiguring a whole CLB column will only affect the LUTs of the SA and not the rest of the system.

\section{Automated placement}

There are several ways to apply these constraints. In the previous approach, placement restrictions were applied by using pblock constraints on the User Constraints File (UCF). These constraints define an area of the FPGA where all logic elements of a certain submodule should be placed. However, the current 
approach requires specifying the exact position of each LUT, so the use of a pblock does not fit this approach.

UCF also allows specifying placement constraints for individual elements, which is better suited to this design. Each of these constraints would specify the hierarchical name of the element within the design and its position in the FPGA fabric. An example of such a constraint would be:

INST "sysarr_system_0/sysarr_system_0/USER_LOGIC_I/SYSTEM_INST/ SYSARR_INST/PE_I_GEN[0].PE_J_GEN[0].PE_INST/STAGE1_GEN [7].LUT6_ INST" LOC="SLICE_X76Y137" | BEL="D6LUT";

where LOC specifies the coordinates of the slice within the FPGA (location) and BEL identifies the basic element within the slice, i.e. which of the four LUTs is to be used. [Xilinx-UG625] provides detailed information of the meaning of these parameters.

However, the number of LUTs is very large: an SA with a size of $4 \times 4$ PEs contains 416 LUTs, which implies writing 416 lines on the UCF. Therefore, it is not feasible to write this file manually. A solution could be to generate this file in an automated manner according to a series of parameters. However, it would still be necessary to launch the generation of this file manually, which interferes with the design process. Additionally, UCF is not supported in Vivado, so removing the dependency on this mechanism would facilitate the portability to newer tools.

For these reasons, it has been decided not to use UCF constraints, and replace them with VHDL attributes as described in [Xilinx-UG625]. Xilinx provides a series of custom attributes that can be embedded in the HDL code itself (VHDL or Verilog), which has the following advantages:

- They are embedded in the module's HDL itself, not requiring external dependencies.

- They refer to specific elements within a certain HDL file, not requiring to specify the whole hierarchy path, which is difficult to generate in an automated manner and depends on the IP core instance name.

- Most importantly, they can be automatically generated from the HDL according to some parameters.

Thus, the aforementioned line would be replaced by a specification like: 


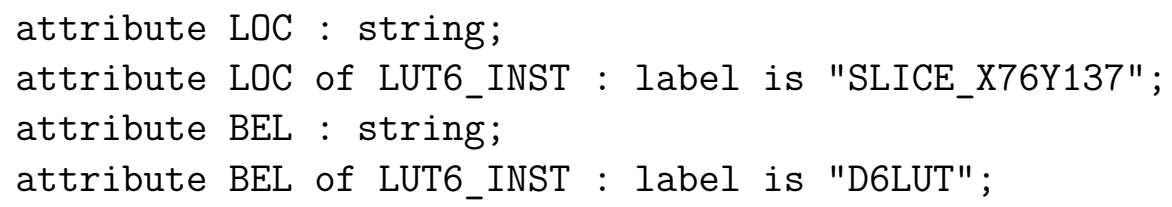

The strings "SLICE_X76Y137" and "D6LUT" can be replaced by expressions that generate the required constraint. For example, the former can be replaced with "SLICE_X" \& natural'image(x) \& "Y" \& natural'image(y), where $\mathrm{x}$ and $\mathrm{y}$ are two constants that are calculated from the entity parameters in the architecture preamble.

Following this methodology, the SA hardware module has been designed in such a way that the user only has to specify the slice position of the top left corner of the array (as shown in Figure 5-11), and the constraints needed to place all the LUTs will be generated automatically.

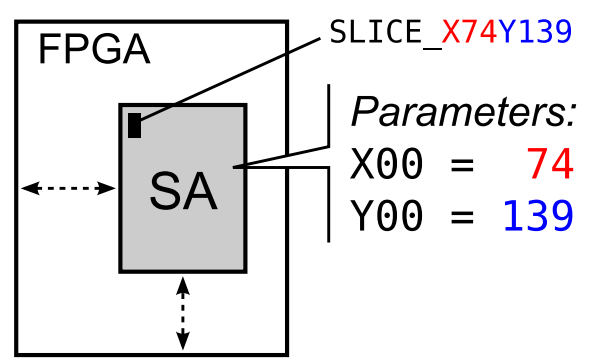

Figure 5-11: Specification of the SA position within the FPGA based on the top left slice coordinates

Specifically, the array has been designed so that a single 8-bit PE occupies two CLBs, with stage 1 using the bottom slices and stage 2 the top ones. This way, a whole PE column can be configured at once in two reconfiguration sequences—one for stage 1 and one for stage 2 .

Original contribution 5-3 The automated generation of placement constraints via VHDL attributes, removing the need of generating a complicated constraints file (manually or automatically). 


\section{Placement prohibition}

As it was explained in $\S 4.1$, the reconfiguration engine reconfigures whole CLB columns. Therefore, the design has to avoid placing other logic elements in the CLB columns belonging to the SA, as these elements would be overwritten by the reconfiguration engine.

The problem is that the SA may not occupy the whole height of the CLB column: for example, an $8 \times 8-\mathrm{PE} \mathrm{SA}$ where each PE or input selector is two CLBs high will use 18 of the 20 CLBs in a Virtex- 5 column. Therefore, the place and route process may decide to place logic resources belonging to other system components in the two remaining CLBs. While this should not be a problem for FFs as they're not reconfigured, overwriting a LUT belonging to an unrelated part of the system would have unpredictable consequences.

Therefore, this design must prevent the remaining LUTs in the CLB column from being used.

An easy way to achieve this is using the UCF. Xilinx provides a CONFIG PROHIBIT constraint that prevents a range of logic resources from being used. A preliminary approach has been to write this constraint manually in the UCF: since this constraint works with ranges of positions, it does not need to be repeated for a large number of LUTs-just a few constraints suffice to remove all conflicting LUTs in the current implementation. However, as it was said, relying on UCF interferes with the design process, so using HDL constraints would be preferable. Unfortunately, prohibit constraints can be assigned only through the UCF [Xilinx-UG625, § Slices Prohibited].

The chosen solution has been to saturate the remaining space in the reconfigurable area by adding a number of "padding" LUTs whose inputs and outputs are unconnected.

These padding LUTs have been placed by determining the range where the SA will be placed, the total number of LUTs in that area, and the number of LUTs that will be actually used by the SA itself; and then adding the remaining number of LUTs using a LOC parameter that specifies a range of slices. ${ }^{6}$

This parameter-based generation of placement restrictions allows the IP core to be used without any intervention by the user other than specifying the array position within the FPGA through the module parameters.

\footnotetext{
${ }^{6}$ This "slice range" feature is not available in Vivado [Xilinx'14]. An alternative is to generate all the LUT positions in the reconfigurable area, and instantiate a padding LUT in each position that is not used by the SA.
} 
Original contribution 5-4 The use of padding LUTs as a replacement for placement prohibit constraints.

Original contribution 5-5 A scalable SA design as a fully parameterized module (with all the necessary placement constraints built into it).

\subsubsection{Reconfiguring a LUT-based design}

\section{Application-specific reconfiguration peripheral}

Reconfiguration of the described design is done using a tailored peripheral IP core that uses the fast reconfiguration engine described in $\$ 4.1$.

This peripheral simplifies the interface between the fast reconfiguration engine and the microprocessor by providing a layer of software-accessible registers. These registers are used to specify an FPGA frame address and partial bitstream memory addresses, as well as the number of frames to reconfigure. The remaining configuration parameters (number of cells and height of each cell) are fixed.

These parameters allow reconfiguring a single column of LUTs. The software application will be in charge of sequencing requests to this peripheral in order to set a specific configuration.

The peripheral needs to store 16 PBSs for the 16 different PE functions, plus 9 for the input selectors (one for each of the 9 possible pixels on the $3 \times 3$ window), and 2 for the multiple segments of the output selector (which can be configured to take their input from the PE to the left or the segment above). Therefore, PBSs for a total of 27 elements need to be stored. Additionally, since each PE has two stages which require two different PBSs, the number of partial bitstreams doubles.

In order to fit these many PBSs, the bitstream memory has been partitioned into 64 equal parts. This way, only 6 bits need to be transmitted from the microprocessor to the IP core in order to specify a certain PBS, allowing to transmit up to 5 addresses simultaneously on a single 32-bit register; the IP will expand these shortened addresses by appending zeros, concatenate them, and pass them to the reconfiguration module from $\S 4$.1.3.

Since one PE or input/output selector is 2 CLBs high, a 20 CLB column is divided into 10 cells. The parameters passed to the reconfiguration IP core via registers are the 6-bit addresses of the PBSs for the 10 cells (which fit in 
two 32-bit registers), a 24-bit frame address, and the number of configuration words to write, as shown in Table 5-5. Writing to register 0 also triggers the reconfiguration process. Additionally, reading from this register provides status information, indicating whether the reconfiguration has finished and if the peripheral is ready to receive a new reconfiguration request.

\begin{tabular}{c||c|c|c|c|c|c|} 
Bits & 3130 & $29 \ldots 24$ & $23 \ldots 18$ & $17 \ldots 12$ & $11 \ldots 6$ & $5 \ldots 0$ \\
\hline \hline Register 0 & \multicolumn{2}{||}{ word count } & \multicolumn{4}{|c|}{ frame address } \\
\hline Register 1 & - & cell 9 & cell 8 & cell 7 & cell 6 & cell 5 \\
\hline Register 2 & - & cell 4 & cell 3 & cell 2 & cell 1 & cell 0 \\
\hline
\end{tabular}

Table 5-5: Register description for the reconfiguration peripheral for LUT-based PEs. This peripheral is designed to reconfigure a CLB column divided into 10 cells, from 0 (bottom) to 9 (top).

Given the small size of the PBSs, 4 words for stage 1 and 8 for stage 2, the partitioning can be done in blocks of 8 words, so the 64 PBSs require a minimum memory size of 512 words (2048 bytes), thus fitting on a single $4 \mathrm{~KB}$ RAMB36 primitive.

\section{Software application}

On the processor's side, the possible values of the register containing frame address and number of words is tabulated, so this value can be obtained directly by a table look up. This is more efficient than generating the value on the fly by concatenating values, or writing to multiple registers.

In order to optimize the write of registers 1 and 2 as well, the EA in software has been designed so that a chromosome stores the value for these two registers directly, for each of the columns the SA spans. ${ }^{7}$ The mutation function will be in charge of modifying the 6 bits of the chromosome corresponding to a cell containing a single $\mathrm{PE}$ or selector ${ }^{8}$ using bitwise operations when a modification of a gene is required by the algorithm.

The reconfiguration process is accelerated by keeping track in software of the previous configuration of the SA, and only calling the reconfiguration

\footnotetext{
${ }^{7}$ For example, the chromosome for an SA spanning 10 columns will contain 20 configuration words corresponding to the addresses of stage 1 ; those for stage 2 will be obtained by setting the lowest bit of each address to 1 .

${ }^{8}$ The exception to this is the output selector, whose modification involves modifying the configuration of multiple cells. This is still considered as a single gene modification from the point of view of the EA; the implementation takes care of modifying all the needed fragments of the chromosome when this gene is modified.
} 
peripheral for those columns whose configuration has changed, this is, whose two configuration words (for registers 1 and 2) differ from the stored ones. Since the EA only makes small modifications on the chromosome, only a few columns will need to be reconfigured.

\subsubsection{Analysis of the solution and experimental results}

\section{Behavior}

The correct behavior of the obtained implementation has been assessed by comparing it with a simulated model that will be described in $\$$ 5.2.1.

The experiment has consisted in launching a set of evolutionary processes with the same parameters, initial random seeds, and training image set both in hardware and simulation. It was observed that both hardware and simulation reached the same fitness at the end of the evolution, indicating that the behavior of the hardware implementation is exactly the expected one.

In order to further prove the correctness of the hardware implementation, a checksum of all the intermediate evaluations was calculated. This checksum also turned out to be identical for both hardware and simulation, indicating that not only both evolutions reached the same result, but also that they showed the same results for every candidate solution that was evaluated. Therefore, it is concluded that the LUT-based implementation behaves correctly and identically to the previous one.

\section{Resource usage}

Each PE uses a total of 16 LUTs, 2 four-bit carry chains, and 9 FFs (one of them used exclusively as pass-through due to routing restrictions). The design methodology has allowed to place these elements in a tightly packed manner, in such a way that a PE fits entirely in 2 CLBs, and that the local LUT usage in the SA area is $100 \%$. This compactness keeps interconnections short (Figure 5-12), which results in a better timing behavior.

Each input selector uses 16 LUTs and 48 FFs (including the registers used for pipelining), and each stage of the output selector uses 8 LUTs and 8 FFs. Additionally, padding LUTs are added to ensure that no extra logic is placed in the area to be reconfigured ( $\$ 5.1 .4)$. Therefore, an $8 \times 8 \mathrm{PE}$ array spans a total area of 200 CLBs (20 CLBs vertically and 10 horizontally, including two CLB columns for the input and output selectors), and uses 1600 LUTs (padding included), 1408 FFs, and 128 carry chains. 


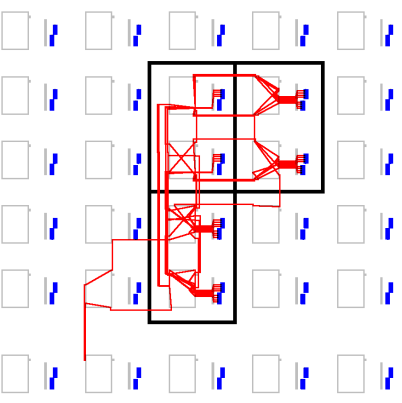

Figure 5-12: Detail of the interconnections between a PE (top left) and its two neighbors connected to its output (right and bottom) as seen on FPGA Editor. Regardless of the array size, the interconnections between neighbors are kept short.

Overall, the hardware peripheral containing the $8 \times 8$ SA uses 2598 LUTs, $2184 \mathrm{FFs}$, and 12 BRAM blocks; and the reconfiguration peripheral uses 656 LUTs, 462 FFs, and 1 BRAM block. This represents less than $5 \%$ of the LUT/FF resources available on the Virtex-5 LX110T used. Therefore, this implementation brings the opportunity of integrating this module into larger systems, or incrementing the number of SAs in order to perform a parallelized evaluation of candidate solutions (as will be done in $\$ 6.2$ ).

As can be seen, although this implementation has the drawback of requiring additional resources for the reconfiguration peripheral, its small size (less than $1 \%$ of the FPGA) makes DPR readily become advantageous with respect to other solutions such as VRC.

\section{Speed}

Once the correct behavior of the implementation has been assessed, it was measured how fast it could perform.

The first measure was theoretical, and was obtained from Xilinx ISE's timing report. This report indicates that the systolic array can work at a maximum frequency of $350 \mathrm{MHz}$.

Nevertheless, this is a rather conservative report that shows a worst-case behavior of the system. In $\$ 5.3 .5$ it will be seen how this array can be safely overclocked to achieve operating frequencies of 400 and up to $500 \mathrm{MHz}$. 


\section{Size}

Thanks to the parameterization of the system and the automated placement of elements, it has been possible to scale the SA to very large sizes. The array has been tested for sizes of up to $\mathbf{2 4 \times 2 4}$ PEs (Figure 5-13).

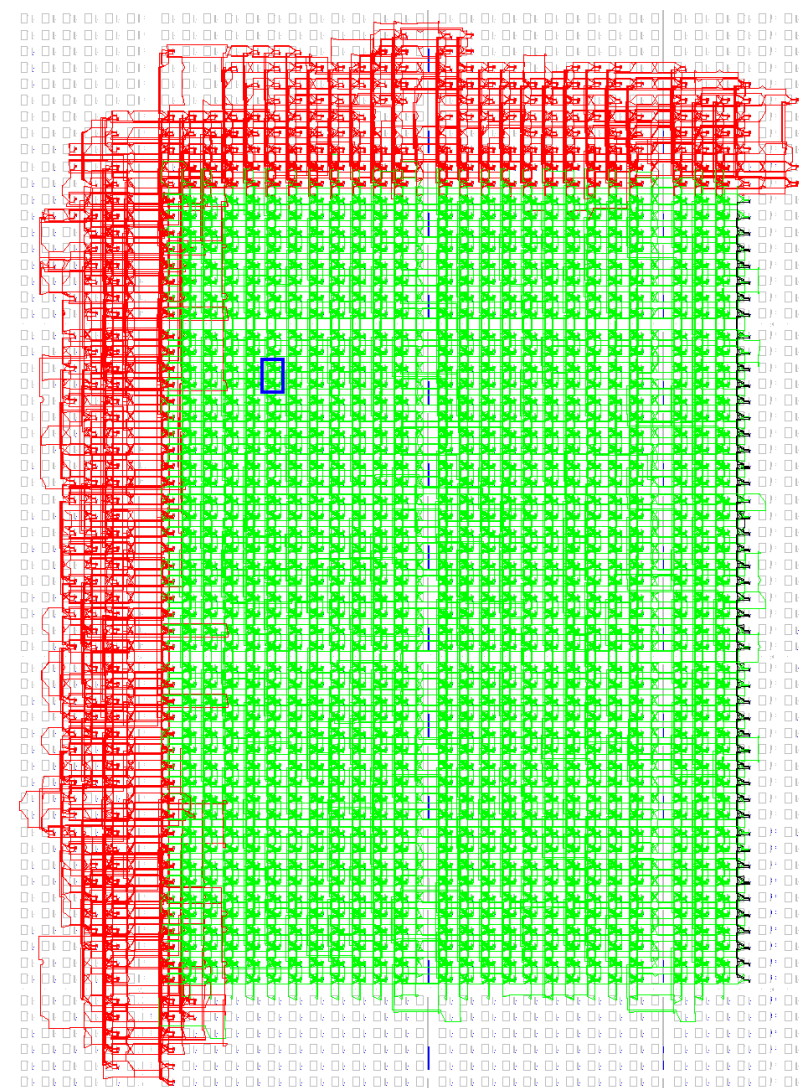

Figure 5-13: Screen capture of Xilinx FPGA Editor showing a $24 \times 24$ array. Red: input selectors, including pipelining. Green: $24 \times 24 \mathrm{PEs}$, with an individual PE marked with a blue box. Black: output selector.

This increase in size does not affect the operating frequency of the array because the length of the interconnections between neighbor PEs is not affected by the overall size of the SA since they always connect nearby elements, as it was seen in Figure 5-12. 


\subsection{COMPARISON OF DIFFERENT RECONFIGURABLE ARCHITECTURES: CGP AND SA}

The choice of the SA architecture over other alternatives has been motivated by previous work, in which this topology was chosen due to its simplicity of interconnections and easy scalability. However, as it was discussed in $§ 2.2 .2$, there are several alternative EHW topologies in use in the state of the art.

One of the most popular topologies for EHW is CGP [Miller'11, Vašíček'07b, Glette'08, Li'09]. This popularity arises from the fact that arbitrary logic circuits can be mapped to it, by implementing a logic gate on each PE and configuring the PE inputs to replicate the routing of the circuit. That way, CGP is a very direct implementation of the idea of evolvable hardware: a digital circuit that can be modified and evolved at the logic gate level. This allows designing digital circuits that can later be implemented directly. In order to simplify the evolution and reduce the search space, CGP can also be used with more complex PEs such as adders or subtractors.

However, CGP has an important disadvantage: it needs to use multiplexers in order to select the inputs for each PE. Multiplexers are themselves logic elements, and as such do use resources when implemented on an FPGA. This is not a problem if the FPGA is only being used to obtain an evolved circuit which will later be implemented directly without multiplexers, either on an FPGA or an ASIC; however, if it is planned to use the same architecture for both evolution and runtime operation, having larger PEs will involve a higher resource usage, which could limit the size of the processing architecture.

SA provides a solution to this problem by fixing and limiting the connections. This way, each PE is connected exclusively to its neighbors in a fixed way, thus removing the need for multiplexers at its input. Additionally, this also simplifies the routing of interconnections between PEs, making them local and therefore shorter, thus promoting a good timing behavior.

By removing the input multiplexers, each individual PE will use fewer resources than that of CGP, which allows making bigger and more complex architectures (or similar in size, but larger in number) when the amount of resources is limited. However, this is a double-edged sword, since the reduced interconnection possibilities limit the potential computational power of these architectures, so it would be expected that the performance of an SA is worse than that of a CGP array with the same number of PEs. 
The question is: is it worth having PEs that use more resources for the sake of connectivity, or will this connectivity not represent an important advantage? Which topology is better, CGP or SA? And more importantly, how does this difference vary as the architecture is scaled in size?

In order to determine this dilemma, an analysis has been carried out, comparing the performance of each of these architectures for the main application this thesis revolves around: image filtering. The results of this analysis have led to the publication of a journal article in Genetic Programming and Evolvable Machines [Mora'18a].

Original contribution 5-6 A comparative analysis between CGP and SA in terms of performance and resource usage with scalability.

\subsubsection{Implementation details}

The details of the implementation of the CGP- and SA-based systems and test conditions that have been used for this comparative study are described below.

\section{Simulation platform}

In order to study the behavior of different architectures without needing to implement them in hardware (with all the development time this would involve), a simulation program has been developed. This program has been written in $\mathrm{C}$ and is designed to be functionally identical to implemented designs, both in the evolutionary algorithm and the computing functionality.

Versions of the simulation software have been developed for both CGP and SA architectures. However, this software is considerably slower than the hardware implementations, more so the larger the processing array is, since increasing the array size involves performing more calculations. These calculations are performed in parallel in hardware implementations, thus not affecting the evaluation time; but sequentially in software. Therefore, for an array twice as large horizontally and vertically, it is expected that the evaluation time be about 4 times longer.

An alternative to accelerate this would be to implement both architectures in hardware. However, implementing a reconfigurable CGP architecture in hardware is beyond the scope of this thesis, so CGP has been evaluated using the software platform. 
SA, on the other hand, has been evaluated using a hardware implementation in order to reduce the evaluation time (not without previously validating that hardware and software versions of SA behave identically). This implementation is based on a $24 \times 24 \mathrm{PE}$ array, and has been used to evaluate the behavior of SA sizes from $4 \times 4$ to $24 \times 24$ PEs. ${ }^{9}$ Such a large array has been possible to implement thanks to the system parameterization and important reduction in resource usage described in $\$ 5.1$.

Original contribution 5-7 This simulation platform is a contribution of this thesis, and could be useful either for verifying future changes to the HW platform or for implementing a heterogeneous computation platform that uses both the FPGA and software implementations as accelerators [Fernández'14, Niño'15].

\section{Architecture}

Both architectures behave in a purely functional way, this is, they are pipelined in such a way that the output at a certain time is a function of the input a fixed number of clock cycles before, without data from inputs at different clock cycles being mixed, as detailed in $\$ 5.1 .3$ (in the case of the computer model, this is implemented by not emulating latency in any way, and just calculating the output from the inputs for every pixel).

The set of functions implemented by the PEs in the case of SA is the one described in $\$ 3.1 .1$ (Table 3-1) as proposed by [Salvador'11a]. However, CGP uses a reduced version of this set, since many of them are redundant there-for example, $N-W$ and $W-N$ correspond to a single $a-b$ function. The list of PE functions for CGP are shown in Table 5-6.

Both SA and CGP have been tested with a varying number of PEs. In the case of SA, all tested sizes have the same number of rows and columns, with sizes ranging from $4 \times 4$ to $24 \times 24$ PEs (nevertheless, in [Gallego' $13 \mathrm{c}$ ] it is shown that other ratios between rows and columns have a similar performance). CGP uses twice as many columns (stages) as rows (PEs per stage), as this ratio has been frequently used in previous work [Sekanina'03, Dobai'14].

Each CGP PE can take its input from either the primary input $(3 \times 3$ window) or any of the PEs on the column immediately to its left.

\footnotetext{
${ }^{9}$ The behavior of smaller SAs is emulated by using a small fragment of this array and filling the unused PEs with pass-through elements.
} 


\begin{tabular}{ccl} 
Index & Equation & Description \\
\hline 0 & 255 & Constant value of 255 \\
1 & $a+b \bmod 256$ & Addition (modulo 256) \\
2 & $\min (a+b, 255)$ & Addition (limited at 255) \\
3 & $\max (a-b, 0)$ & Subtraction (limited at 0) \\
4 & $\left\lfloor\frac{a+b}{2}\right\rfloor$ & Average (rounded down) \\
5 & $\left\lfloor\frac{a}{2}\right\rfloor$ & Divide by 2 (rounded down) \\
6 & $\max (a, b)$ & Maximum \\
7 & $\min (a, b)$ & Minimum
\end{tabular}

Table 5-6: Functions implemented by the PEs in CGP

As it was shown in $\$ 5.1$, each of these functions requires 16 LUTs to be implemented. However, as it was said before, PEs in CGP require two 8bit multiplexers on their inputs, which also account for the resource usage. In particular, a 16:1 one-bit multiplexer-needed for CGP heights of up to 7 PEs $^{10}$ _requires 4 LUTs to be implemented [Xilinx-UG190, ch. 5]. Therefore, a single PE in CGP will require a total of 80 CLBs: 16 for the function itself, and 32 for each of the two input multiplexers (4 LUTs per bit for 8 bits word length).

SA does not need these multiplexers on every stage; only at the input and output. The reconfigurable selectors described in $\$ 5.1 .3$ demand only 16 LUTs for each input, plus an additional $8 \cdot H$ for the output selector.

A similar approach could have been applied to CGP, replacing the multiplexers with fixed selectors, which would reduce the number of LUTs used for this as it would allow using all 6 inputs of the LUT for data, rather than 4 for data and 2 for selection. However, this would be beyond the scope of this thesis; additionally, the savings would be small—the LUT usage would only be reduced by about $20 \%$.

\footnotetext{
${ }^{10}$ Each PE input on CLB can be taken from any of the 9 pixels on the primary input or the $H$ outputs of the previous computing column. Therefore, for $H=4$ to 7 , eight 13:1 to 16:1 onebit multiplexers are needed, which require 4 LUTs per bit to be implemented [Xilinx-UG190, ch. 5].
} 


\section{Evolutionary algorithm}

The evolution for both architectures has been performed using a simple EA. This EA was used in [Mora'15], and is the result of several improvements that will be detailed in $\$ 6.1$.

The evolution is carried out in 8 separate runs. $^{11}$ Each run employs a simple $(1+1)$ algorithm, in which an individual is iteratively mutated and compared with the original, reverting the changes in case the fitness worsened. Every 2048 generations, the worst run is terminated and the best one is forked in order to improve the evolution results as a tradeoff between the survival of the fittest individual but with sufficient diversity. The evolution is finished after 16 such exchanges, performing a total of 262144 candidate solution evaluations. ${ }^{12}$

The evolution employs a mutation rate of $K=3$, this is, 3 randomly chosen genes are replaced by random values. In the case of SA, each gene represents either the function of a PE (from 0 to 15), the configuration of an input multiplexer (from 0 to 8 , for each of the 9 pixels on the $3 \times 3$ input window), or the configuration of the output multiplexer (from 0 to $H-1$ where $H$ is the height of the SA in PE rows). For CGP, each gene encodes either the function of a PE (from 0 to 7), the configuration of each of the PE inputs (from 0 to 8 on the first column, and from 0 to $8+H$ for the rest), or the configuration of the output multiplexer (from 0 to $H-1$ ), Thus, the total number of genes of an $H \times W$ array is $H \cdot W+H+W+1$ for SA and $3 \cdot H \cdot W+1$ for CGP, respectively.

For the experiments, a $128 \times 128$ pixel grayscale Lena image with a $20 \%$ salt and pepper noise has been used (Figure 5-14). The fitness is calculated as the sum of absolute errors (SAE) between the reference (noiseless) image and the result of filtering the input (noisy) image with the candidate solution.

The resulting fitness of the evolution has been used as measure for the quality of each solution, as it has already been shown in $\$ 3.3$ that the result of an evolution performed with a certain image is generalizable to other images with a similar type of noise.

In order to obtain results that are statistically significant, the whole evolution process is repeated 100 times with different initial random seeds,

\footnotetext{
${ }^{11}$ In $\$ 6.2$ it will be seen that the speed is maximized with 12 SAs in parallel, motivating the use of 12 parallel runs in $\$ 6.1$ rather than 8 . However, the work in [Mora'15] and the studies performed in this section were done before finding this optimal strategy.

${ }^{12}$ The use of powers of 2 is motivated by the method used to find the best evolution parameters, in which an evolution with a fixed size of $2^{18}=262144$ evaluations was partitioned into an optimal number of runs and exchanges. Using powers of 2 simplified the empirical search of this optimum.
} 

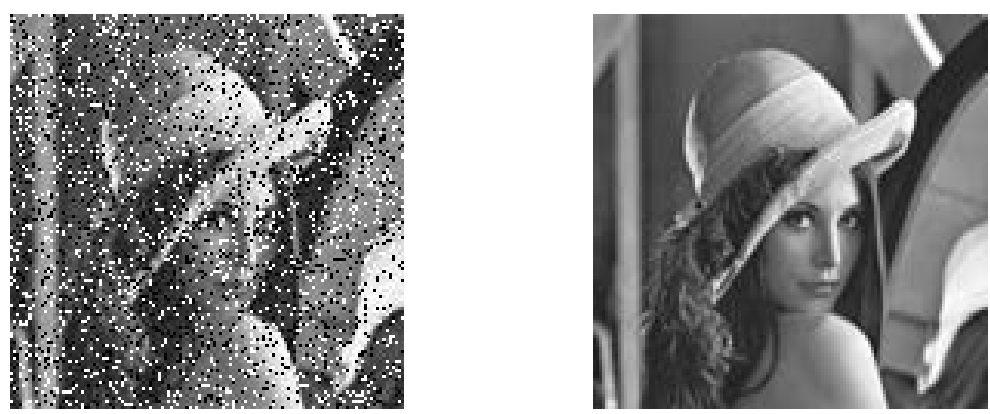

Figure 5-14: Training images used in the evolution: input (left) and reference (right).

measuring the median and 25th and 75th percentiles. These values gives an idea of the average behavior of each solution and the spread of the results.

\subsubsection{Resource usage analysis}

The amount of resources required for the processing array will depend on the size of the array and the topology used. These resources are LUTs (logic elements) and FFs (registers). However, the number of FFs required is very small (8 per PE for both architectures, much fewer than the number of LUTs used in both cases), while there are as many LUTs as FFs on Virtex-5 FPGAs. Therefore, the limiting factor when designing large arrays will be the number of LUTs, not FFs. For this reason, the measures shown in this section only show the LUT usage.

As it has been detailed, PEs in the CGP implementation require 80 LUTs. The output multiplexer will only require one or two LUTs per bit depending on the number of PEs on the last stage, so it has been neglected. Therefore, the total LUT usage of the described CGP architecture is

$$
\operatorname{LUTs}_{\mathrm{CGP}}=80 \cdot H \cdot W
$$

SA, on the other hand, requires only 16 LUTs per PE, 16 LUTs per input selector, and $8 \cdot H$ for the output selector. Thus, the LUT usage for this topology is

$$
\operatorname{LUTs}_{\mathrm{SA}}=16 \cdot H \cdot W+16 \cdot(H+W)+8 \cdot H
$$

Figure 5-15 shows the resource usage of both architectures for different sizes, with SA being square $(W=H)$ and CGP being twice as wide as high $(W=2 \cdot H)$, as is frequently seen on the literature. Numeric values for a few of these sizes are provided in Table 5-7. 


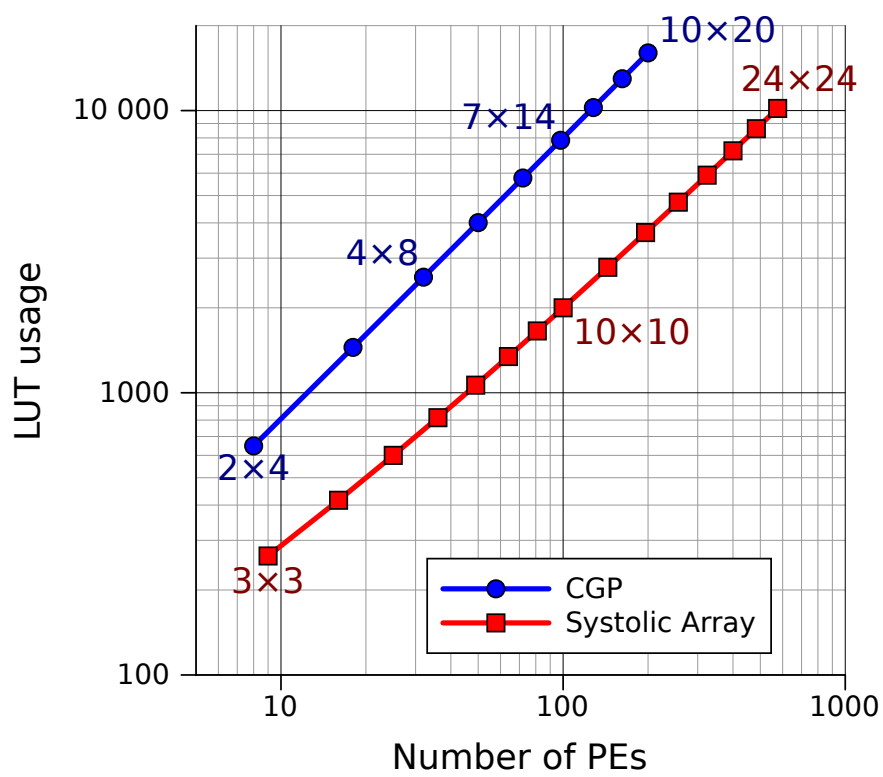

Figure 5-15: Comparison of resource usage versus number of PEs for both SA and CGP.

\begin{tabular}{|c|c|c|c|c|c|c|c|}
\hline \multicolumn{4}{|c|}{ CGP } & \multicolumn{4}{|c|}{ SA } \\
\hline Size & PEs & LUTs & LUTs/PE & Size & PES & LUTs & LUTs/PE \\
\hline $3 \times 6$ & 18 & 1440 & 80.0 & $4 \times 4$ & 16 & 416 & 26.0 \\
\hline $4 \times 8$ & 32 & 2560 & 80.0 & $6 \times 6$ & 36 & 816 & 22.7 \\
\hline $5 \times 10$ & 50 & 4000 & 80.0 & $8 \times 8$ & 64 & 1344 & 21.0 \\
\hline $7 \times 14$ & 98 & 7840 & 80.0 & $10 \times 10$ & 100 & 2000 & 20.0 \\
\hline $10 \times 20$ & 200 & 16000 & 80.0 & $14 \times 14$ & 196 & 3696 & 18.9 \\
\hline $16 \times 32$ & 512 & 40960 & 80.0 & $24 \times 24$ & 576 & 10176 & 17.7 \\
\hline
\end{tabular}

Table 5-7: Resource usage of CGP and SA at different sizes

As it can be seen, SA achieves about 4 times more PEs than CGP for the same resource usage, or 4 times fewer resources for the same number of PEs (specially for large SA sizes, where the resource overhead caused by input and output selectors is proportionally smaller), so this will likely compensate the worse interconnectivity between PEs of SA compared to CGP.

These measures on the resource usage do not take into account the resources used by the rest of the logic (fitness computation, memory interfaces, control modules...). However, these elements do not depend on the array size, so their resource usage is fixed regardless of the size. 


\subsubsection{Evolution results}

Figure 5-16 shows the variation of the fitness for SA and CGP with different sizes (i.e., number of PEs). As it was expected, CGP offers better performance per PE than SA, as the increased connectivity of CGP offers more possibilities for the same number of PEs.

However, when evaluating the results from a point of view of resource usage (Figure 5-17), it can be seen that SA is much more efficient than CGP for moderate sizes. However, SA scales worse than CGP, getting stalled past sizes of $10 \times 10$ PEs and being eventually outperformed by CGP.

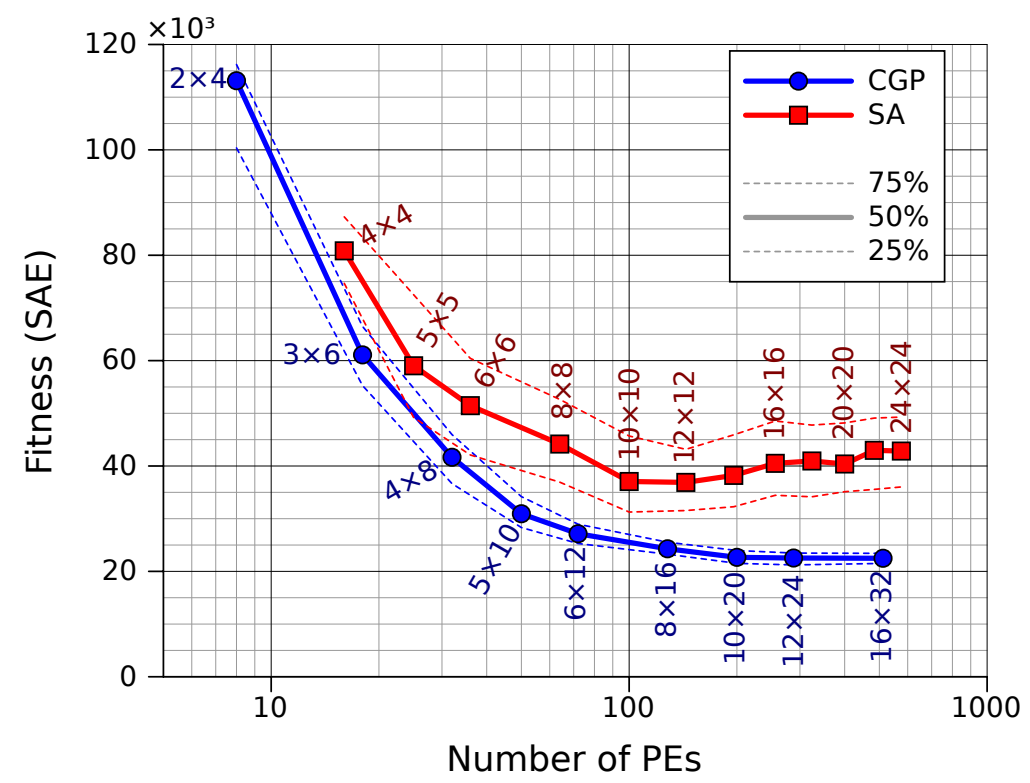

Figure 5-16: Fitness (SAE; lower is better) for different sizes of SA ( $4 \times 4$ to $24 \times 24$ ) and CGP $(2 \times 4$ to $16 \times 32)$. The solid line represents the median of the 100 evolutions for each size, and the dashed lines represent 25 th and 75 th percentiles.

This stalling in the performance can be due to several factors: either the EA is not effective enough to fully exploit the capabilities of large SA architectures, or such capabilities are limited and this limit is reached for a size of $10 \times 10$, with sizes above this not providing any improvement.

CGP eventually reaches a limit too, in this case at a size of $10 \times 20$ PEs; however it manages to reach much better results than SA. Additionally, the dispersion of the results for CGP $(25-75 \%$ distance) is much smaller than that of SA, which may indicate that CGP converges better than SA. 


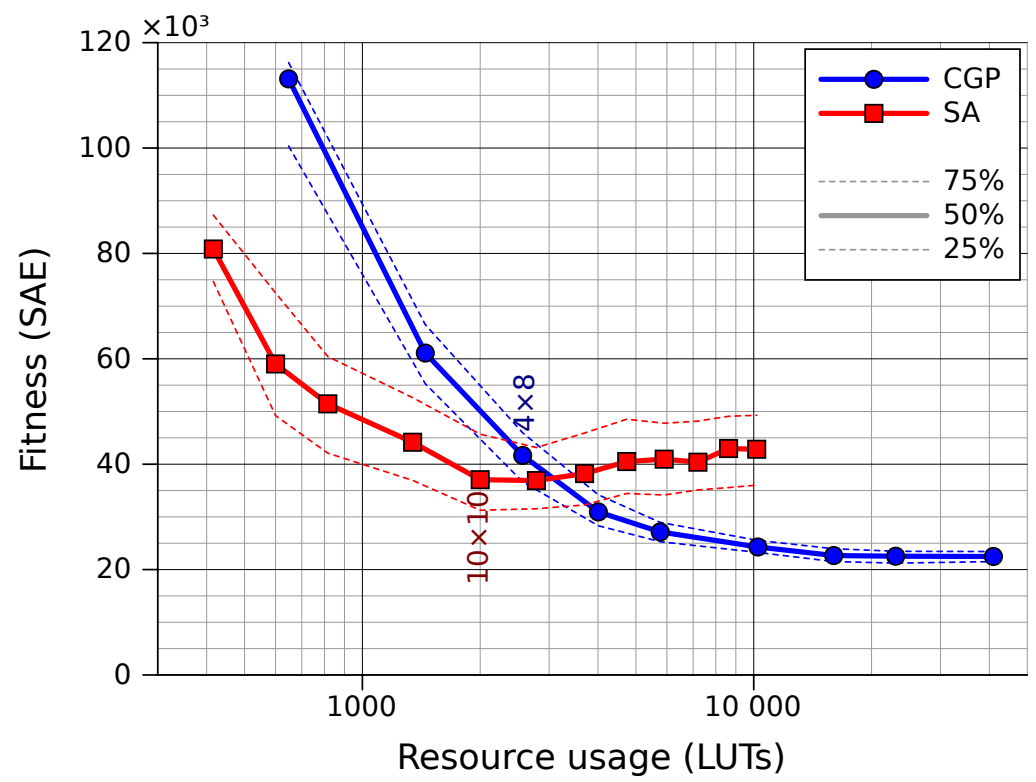

Figure 5-17: Fitness for different sizes of SA and CGP, using LUT usage rather than number of PEs as the horizontal axis.

Original contribution 5-8 The evaluation of scalability in terms of performance versus resource usage as a figure of merit.

\subsubsection{Improving the behavior of SA with scalability}

\section{Evolutionary algorithm}

The behavior of SA on sizes above $10 \times 10$ is somewhat paradoxical: not only does it not show any improvement, but it actually provides worse results. As it was seen in Figure 5-17, the fitness obtained for $24 \times 24$ SA is actually worse than for $10 \times 10$.

This cannot be due to the larger SA having an actually smaller functionality than the smaller one, since a large array can implement the functionality of a smaller array by filling the unused PEs with pass-through functions. Therefore, this behavior indicates a problem in the EA.

A possible reason for SA being less effective than CGP for the same EA is that the search space becomes too large due to the high number of genes. This is not likely the cause of this behavior, since a $16 \times 16$ SA has only 289 genes (where each gene represents either a PE or an input/output selector), whereas an $8 \times 16$ CGP has 385 genes and this behavior is not observable. 
In order to rule out the large number of genes as the cause, the EA has been run for longer times, up to 16 times longer. Figure 5-18 shows that, although a longer evolution time does improve the results, these are still similar to the ones obtained in CGP for a much shorter evolution; and more importantly, it does not solve the problem of scalability for sizes larger than $10 \times 10$ resulting in worse results.

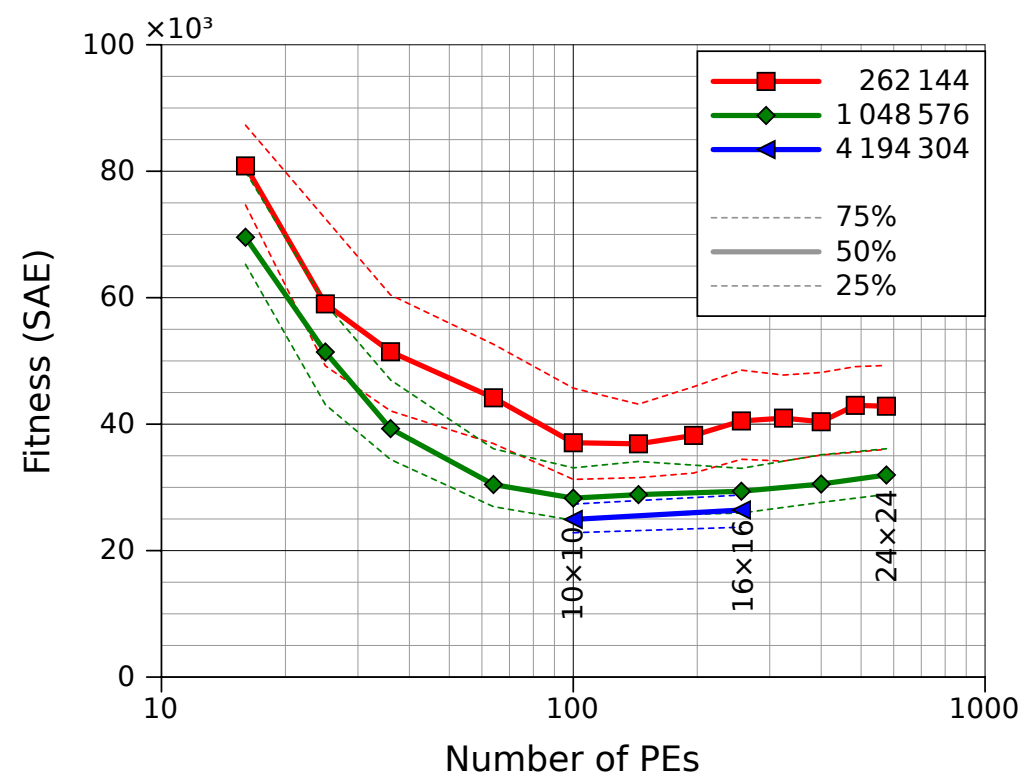

Figure 5-18: Result of having an evolution four times longer, comparing the former length of 262144 evaluations with an extended length of 1048576 . Two points of a 16 times longer evolution (4 194304 evaluations) are shown as well, although the improvement in this case is much smaller.

Another way to overcome the problem of evolving an excessively large genotype is to perform a gradual evolution, starting with a size of $10 \times 10$ PEs and then increasing the array size progressively up to the final size, as it was done in [Gallego'13c]. This will keep the search space small at the beginning of the evolution, "guiding" the EA so that it does not get lost in such a large space.

The results of this approach (for an evolution length of 262144 evaluations) can be seen in Figure 5-19. As it can be seen, this approach does solve the problem of larger sizes resulting in worse performance, and even shows some improvement; however, this improvement is quite small.

The conclusion that can be drawn from this analysis is that both SA and CGP have a limit in the performance that can be obtained, at least with the current EA. This limit is different for both architectures, and attempts to improve the 


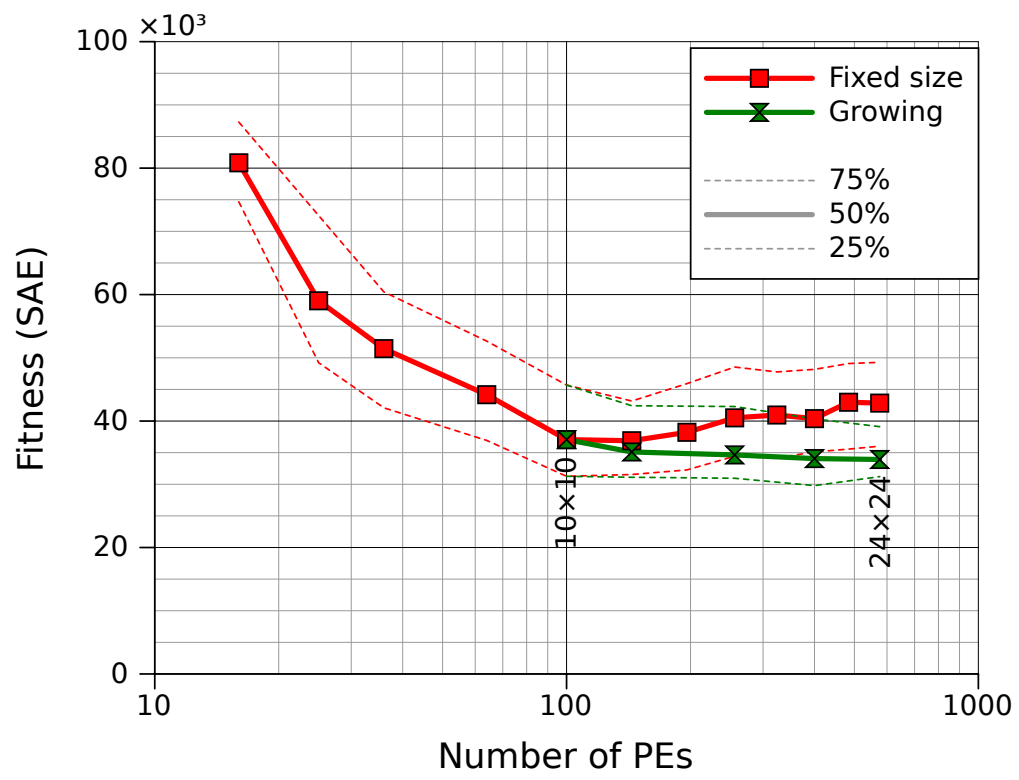

Figure 5-19: Evolution results growing from an initial SA size of $10 \times 10$ to a specific size, compared with the previous results obtained with a fixed size.

behavior of SA were not very profitable. This could mean that the main cause of this difference is the limited connectivity of PEs in SA, not only because it reduces the potential computing capabilities of the architecture but also because it may hamper the evolution.

\section{Improved connectivity through dedicated PEs}

Another approach to improve the behavior of SA for large sizes is to solve the problem of connectivity. The proposal of a new topology with better connectivity is beyond the scope of this thesis; nevertheless, it may be possible to improve the connectivity of the current topology with some minor modifications to the PEs.

One possible reason why CGP outperforms SA for big sizes may be the ability of all PEs to get the primary input in addition to the input from upstream PEs. In an SA, on the other hand, a PE needing the primary input would only be able to get it if said input were propagated through other PEs configured with identity functions (Figure 5-20). This chain of pass-through PEs would not only waste resources, but also act as a barrier preventing other data paths to cross it.

In order to partially address this problem, two new PE functions were originally introduced (Figure 5-21). These new functions differ from the rest 


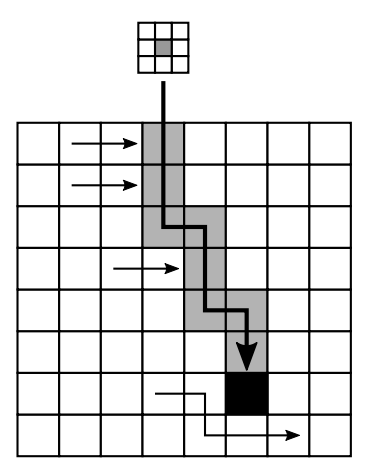

Figure 5-20: One possible way of routing a primary input to an arbitrary PE (marked in black). This requires sacrificing several PEs (marked in gray) and blocks other possible data paths (represented as small arrows).

in that they have two independent east and south outputs. One of the new functions makes the PE act as a "bridge" that crosses its paths, copying the north input to the south and the west to the east; and the other one diverges them, copying north to east and west to south.

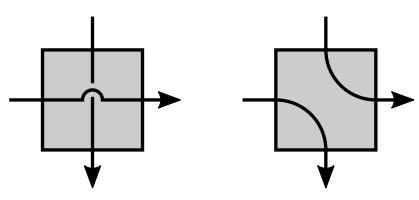

Figure 5-21: New PEs: cross (left) and diverge (right).

These two new functions can be implemented in the current PE architecture with some minor modifications that do not increase the LUT usage (Figure 5-22). However, both outputs need to be registered, which increases the FF usage, although this is not a problem since half of the FFs in the SA area were not used (furthermore, new FPGA families like the 7 Series have twice as many FFs per slice as the Virtex-5). The functionality of the resulting PE is a superset of the old one, so all 16 previous functions can be implemented.

The aim of these two new PEs is to be able to transmit the primary input to a certain PE without acting as a data barrier (Figure 5-23). However, as can be seen in Figure 5-24, although this modification results in some degree of improvement of the results (specially for sizes between $6 \times 6$ and $10 \times 10$ ), the array still reaches its maximum performance at a size of $10 \times 10$. 


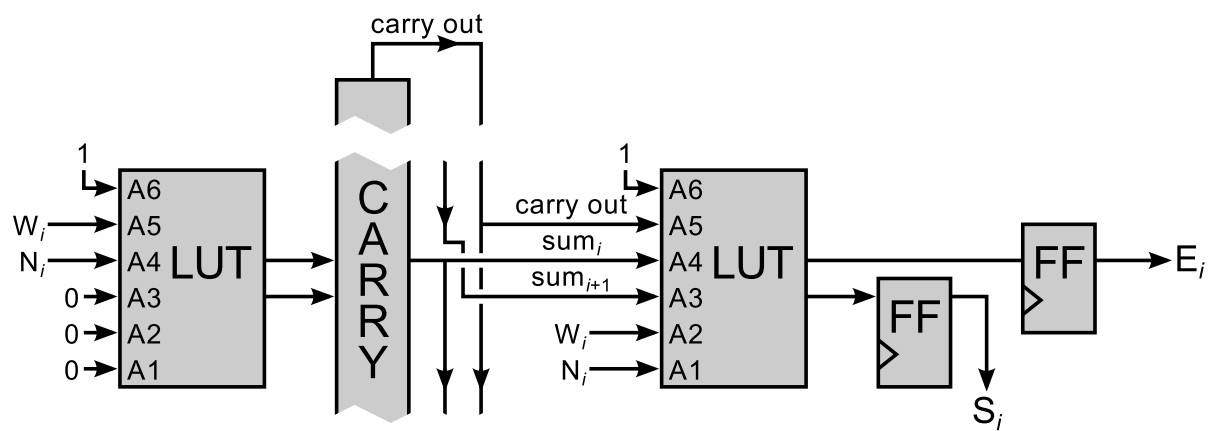

Figure 5-22: Two-output $P E$ architecture

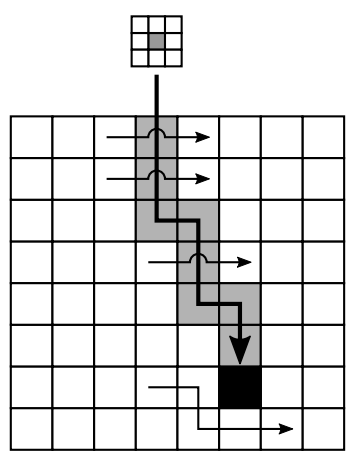

Figure 5-23: With the new PEs, routing a primary input to an arbitrary PE no longer blocks other possible data paths, which are now able to "jump" to the other side, although it still requires sacrificing several PEs for a single PE to be able to access the primary input.

\section{Improved connectivity through bypassable PEs}

The reason why the addition of cross and diverge PEs barely improved the results could be because, as it was seen in Figure 5-23, routing a primary input to a certain PE still requires sacrificing the functionality of several PEs for this connection.

However, following the two-output PE model proposed in the previous point, a different approach could be taken: rather than having two special PE functions with two different outputs, it would be possible to make every PE function able to use one of its inputs as one of the outputs, while the result of the PE function is passed to the other output, as depicted in Figure 5-25. In other words, this makes PEs able to be bypassed, so that they do not necessarily block results from upstream PEs. This approach is actually closer to the one 


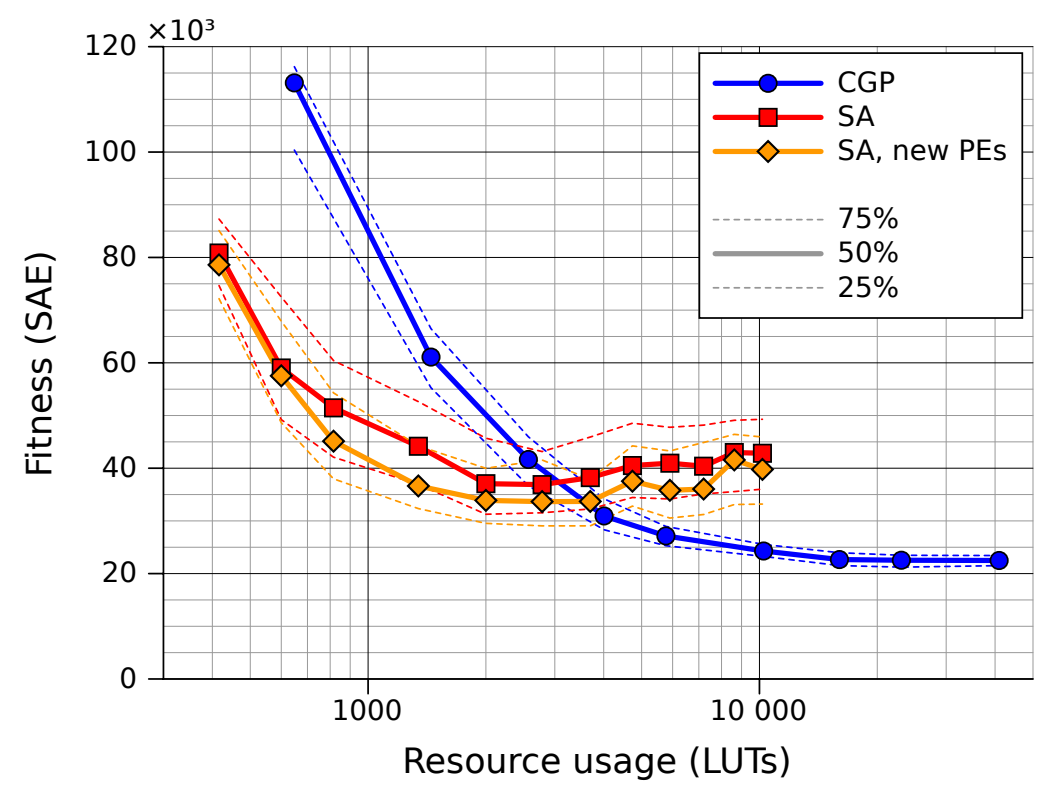

Figure 5-24: Comparison of the results obtained using the former $16 \mathrm{PE}$ functions and the new 18 functions (former 16 plus the two new PEs). The improved system is still unable to reach the performance of the CGP for resource usage above 4000 LUTs.

originally proposed in [Kung'78], where PEs could distribute their data in a pass-through manner or operate on it in a cascaded way.

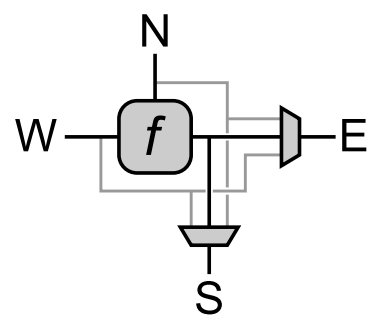

Figure 5-25: Functional diagram of a bypassable PE, where each output can be configured as either the result of the PE function or one of the inputs.

This new PE model can implement the previous 16 functions, as well as the "cross" and "diverge" PEs proposed before, so it is a superset of the previous functionality, with the difference that routing a primary input no longer sacrifices additional PEs, since those can be bypassed while still computing a function for the other output. However, this model is not fully unconstrained, since PEs with an output configured for bypass will be unable to transmit their result to the corresponding neighbor. 
It should be noted that, unlike the multiplexers used in CGP PEs, these are very simple ones that only require selecting between three possible values, so this modification, like the previous one, does not increase the LUT usage and can be implemented in the PE architecture from Figure 5-22. However, it does require a modification in the genotype, and now each $\mathrm{PE}$ is represented by three genes: the function it implements, the configuration of the east output, and the configuration of the south output. ${ }^{13}$ This PE model also makes functions 10 and 11 from Table 3-1 ( $N$ and $W$ pass-through) redundant (since they can be implemented simply by bypassing the PE), so they have been removed, leaving the number of PE functions as 14 .

Figure 5-26 shows the results obtained with this approach. As it can be seen, although there are still some problems with scalability above sizes of $14 \times 14$ (which are likely due to limitations in the EA) for this evolution length, the results are much better than before, showing a better fitness and a much smaller dispersion, comparable to that of CGP.

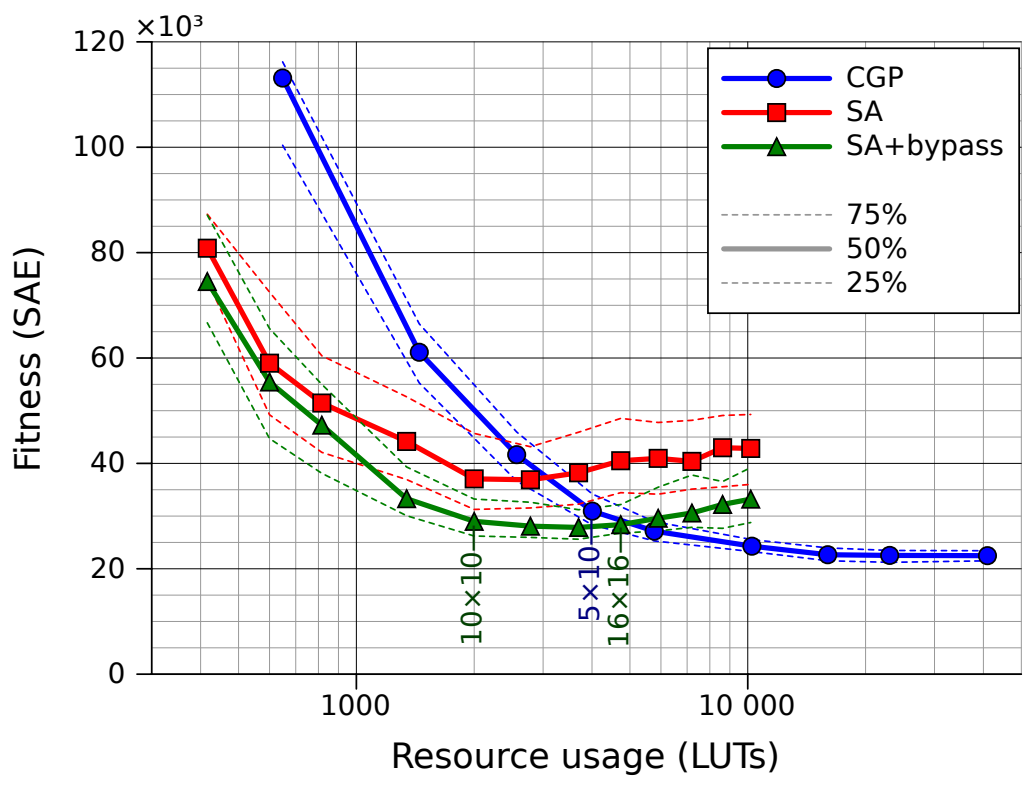

Figure 5-26: Results obtained with the bypassable PEs, compared with the ones obtained with CGP and the initial version of SA. As it can be seen, while the LUT usage for the SA with bypassable PEs is identical to that of the old one, the results are much better.

\footnotetext{
${ }^{13}$ This complicates the reconfiguration methodology, making the capabilities of the reconfiguration engine implementation described in $\$ 5.1 .5$ insufficient, and thus requiring a more complex one in order to reconfigure efficiently. An improved implementation addressing this issue among others will be proposed in $\$ 7.2 .2$.
} 
This modification resolves the main weakness of SA against CGP, making it the preferred option in terms of resource usage for most scenarios. However, although this modification provides a significant improvement over the previous approach, this result has been obtained at a very late stage of the thesis, not allowing for further analysis and exploitation. Additionally, it requires a considerable modification of the preexisting evolution and reconfiguration routines that had already been developed in this thesis, so it is not trivial to adapt it to the already existing use cases. Therefore, this modification has been excluded from the rest of the work shown in this thesis. Nevertheless, it is an interesting candidate for future work.

Furthermore, investigating other possibilities of dual-output PEs providing two "complementary" outputs that can be computed simultaneously without using more resources (such as maximum and minimum ${ }^{14}$ ) could be a potentially profitable future line of development. Said research would fall in the category of improvements on the PE library, and thus should be complemented with a study for the improvement and extension of the current set of $\mathrm{PE}$ functions.

Original contribution 5-9 The use of bypassable PEs is an original contribution in the context of dynamically reconfigurable SA-based architectures. Nevertheless, the original description of $S A$ as a generalized processing topology already considers this possibility.

\subsubsection{Other considerations}

In addition to resource usage and filter performance, there are other factors that have not been experimentally analyzed in this section but that should be considered as well:

Simplicity of design. The lack of scalable multiplexers in the SA architecture simplifies the design. CGP, on the other hand, requires its multiplexer size to depend on the vertical size of the array. Furthermore, these multiplexers require additional control registers to select their functionality. As it was said in $\$ 5.2 .1$, these multiplexers could be replaced by LUT-based reconfigurable selectors; however, designing such selectors in a scalable way would be complicated.

\footnotetext{
${ }^{14}$ Blocks that output the maximum and minimum of two inputs are used in some implementations of median filters [Sekanina'04, fig. 7.4].
} 
Complexity of interconnections. Since interconnections in SA go from one PE to two adjacent ones, they are short, resulting in a very reduced delay and a small routing effort. CGP, on the other hand, requires the output of each PE to reach multiple PEs (as many as there are rows in the array), making these interconnections more complex. In CGP, this complexity in the interconnections grows the larger the array is, whereas in SA the size of the array does not influence the length and complexity of the interconnections.

Dynamic scalability. Previous work [Gallego'13c] demonstrates the possibility of using DPR to make an SA scalable at runtime. This is possible because the structure of SA is homogeneous and the interconnections are simple and immutable. Scaling a CGP architecture vertically would be unfeasible, as it would involve changing the structure of the interconnections. ${ }^{15}$ Horizontal scalability would be easier to achieve as it would just imply adding more stages; however, the complexity of the interconnections would probably require a large space overhead to make routing possible without violating the routing constraints required by DPR.

Usability of preexisting EAs. An advantage of CGP over SA is that it was designed specifically with evolution in mind. Its architecture is designed to be compatible with concepts such as genetic programming, allowing the implementation of functions in the form of trees. Nevertheless, CGP is frequently used with simpler EAs that focus on mutating individual elements of the genotype, and it has been shown that SA performs similarly well in this aspect (with a lower resource usage).

\subsubsection{Conclusions}

In this section, it has been shown that both SA and CGP are good alternatives for the implementation of EA.

From the point of view of resources, SA provides much better results for a low resource usage. However, oversized SAs do not provide a significant improvement over a size of $10 \times 10$ PEs. Therefore, it is reasonable to use SAs with sizes of $8 \times 8-10 \times 10$ for the purpose of evolvable hardware.

\footnotetext{
${ }^{15}$ The author does not consider scalable interconnections to be impossible. For example, these could be implemented as a scalable interconnection matrix that provides a crossbar between stages of CGP. Nevertheless, this would likely be too complex to implement, and the size of such matrix would probably consume an excessively big amount of resources.
} 
The resources saved by using an SA architecture can be invested in implementing multiple arrays (this is, scaling the number of arrays rather than the size of each array). This can be used to accelerate the evolution by evaluating multiple solutions in parallel (as is done in [Dobai'14]), or to filter multiple images simultaneously, thus achieving better performance after the training stage. Having multiple arrays can also be useful as a redundancy measure, ensuring that the system can still work even if a single array fails [Gallego'13b] ${ }^{16}$.

Additionally, multiple arrays may be cascaded in order to obtain a larger filter, rather than scaling the size of a single array. This is demonstrated in [Gallego'13b], and has the advantage that it allows evolving each array separately, thus simplifying the search space considerably.

Finally, a small resource usage may be advantageous if the resources are limited (for example, on low-end FPGAs), or if the EHW is going to be part of a larger system.

All these arguments suggest that the best suited architecture for the kind of EA being studied is an SA of a size of $10 \times 10$ or below. Specifically, arrays of size $\mathbf{8} \times \mathbf{8}$ have shown to be quite practical to use [Mora'15, Mora'18b], since these arrays fit vertically on a single FPGA clock region when using the PE implementation described in $\$ 5.1 .{ }^{17}$ This size provides a good compromise between resource usage, filter performance, and ease of reconfiguration.

\subsection{REDESIGNING THE SYSTEM WITH A METHODOLOGY THAT EMPHASIZES GENERALIZABILITY}

As it was said in $\$ 4.1$, EHW systems, specially those intended to be used in real time applications, need to have a small evolution time. Most of the time

\footnotetext{
${ }^{16}$ The author of this Ph.D. thesis is a coauthor of [Gallego' 13c].

${ }^{17}$ A $10 \times 10$ SA implemented as described in $\$ 5.1$ would overflow an FPGA clock region by 2 CLBs, thus spanning for two clock regions, which will need to be filled with padding LUTs $(\$ 5.1 .4)$, resulting in a waste of resources. Therefore, the height of the SA must be limited to 9 PEs; however, leaving a margin of a few CLBs between SAs in parallelized implementations such as [Mora'15, Mora'18b] may avoid potential routing problems. This motivated the choice of a height of 8 PEs.
} 
spent into evaluating each of the candidate solutions goes into two main tasks: reconfiguring the hardware and using it to process the training input. The reconfiguration engine described in $\$ 4.1$ only covers the former. In order to accelerate the latter, the reconfigurable processing system has to be optimized so that it achieves a throughput as big as possible.

In $§ 5.1$, it has been seen how a LUT-based implementation of the SA can achieve high working frequencies, theoretically up to $350 \mathrm{MHz} .{ }^{18}$ This would provide a filtering throughput of 350 megapixels per second.

However, the array is not the only part of the processing system. Input and output data must be read from and written to memories, the $3 \times 3$ pixel window has to be generated, and fitness must be computed at one pixel per clock cycle as well. Therefore, if these logic units surrounding the SA are slower than the SA itself, they will become the limiting factor for the system speed.

The system implementation described in chapter 3 did not show too good timing behavior, displaying a considerable amount of faults at speeds of $200 \mathrm{MHz}$. Additionally, it was implemented targeting a specific application, hindering its reuse for future applications. Thus, in order to take advantage of the fast and parameterizable SA described in $\$ 5.1$, the system needs to be redesigned using a methodology that favors performance and facilitates its adaptation to new use cases.

\subsubsection{Design considerations}

The system described in chapter 3 was designed for a very specific problem, and its implementation was far from ideal. A more generalizable design that is able to grow and adapt to new problems requires reconsidering the design methodology.

The generalizability of the design will be driven by the following guidelines:

- Make the system modular, in such a way that each module performs a specific task independently of the rest. This will be helpful in the design process and will allow reusing these modules in future designs.

- Parameterize the design. Making the system parameterized will make it more generalizable and reusable, and remove the need of modifying the whole HDL code if different specifications are required.

\footnotetext{
${ }^{18}$ This figure is subject to experimental assessment; it is expected that higher frequencies will be obtained in practice, since this timing report is quite conservative. Furthermore, a small number of timing errors during the training stage would be acceptable, since they will be discarded in the EA.
} 
- Simplify the design. This not only helps during the development and future reuse, but also makes the system have a better timing behavior, as the maximum operational frequency is reduced the more complex the logic operations are.

This way, the system will not be restricted to a single use case-the image noise removal used as proof-of-concept in this thesis-but instead will be suited for numerous different applications.

\subsubsection{Separation of the design into different clock domains}

It is likely that different parts of the system will have different maximum clock frequencies. For instance, although the theoretical maximum FF switching frequency of the Virtex-5 FPGA used in this study is $600 \mathrm{MHz}$ [Xilinx-DS202], BRAMs are limited to $450 \mathrm{MHz}$. Similarly, parts using more complex logic will likely have a slower maximum frequency.

One way to improve the throughput of the slower parts is to have multiple logic blocks working in parallel. For example, the fitness computing unit could use two separate comparators and adders, one for odd pixels and the other for even pixels, thus being able to process two pixels per clock cycle.

However, unnecessarily replicating the logic of the system would result in an excessive resource usage, so this should be done only when strictly necessary. Therefore, the system has been split into regions working at different clock speeds.

These regions share data using dual-clock asymmetric FIFOs. These FIFOs ensure that data can cross clock boundaries without glitches.

"Asymmetric" refers to the fact that input and output ports have different widths. For example, a FIFO designed to transfer data from a region operating at $100 \mathrm{MHz}$ to another one operating at $200 \mathrm{MHz}$ may have a 16-bit input (able to transmit two pixels per clock cycle) and an 8-bit output. Thus, despite having different clocks, both input and output will operate at the same throughput of 200 megapixels per second.

Figure 5-27 shows the system division into different clock domains. The data width of each domain has been parameterized, except for the SA domain which operates at one pixel per clock cycle due to the nature of the SA.

Each clock domain of the design has the ability to pause depending on the status of the surrounding FIFOs, so that data will not be lost or corrupted when these get full or empty. The exception is the SA domain, as the current 


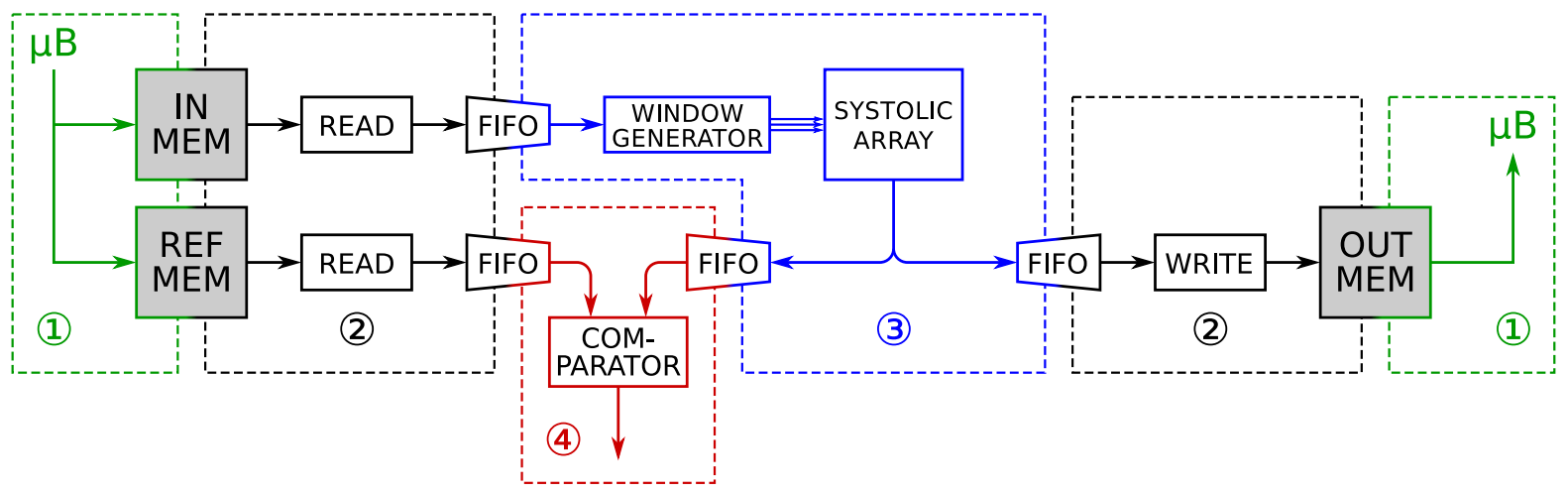

Figure 5-27: Clock domain division of the system, differentiating the clock domains of the MicroBlaze processor (1), the BRAM reading and writing (2), the SA itself (3), and the fitness unit (4), which is able to process multiple pixels in parallel.

implementation of the SA does not have a way to pause the data stream, ${ }^{19}$ and just uses a delay line from the input FIFO to the output one in order to enable the latter. Therefore, care must be taken to ensure the FIFOs at the input of this domain to never run empty, and FIFOs at the output to never get full. This can be achieved by setting the different clocks and data widths of each domain so that the throughput of the SA domain is never greater than that of the other domains.

Original contribution 5-10 The separation of the SA-based processing system into multiple clock domains operating at different clock frequencies in order to obtain the maximum performance on each part.

\subsubsection{Simplifying the design for a better performance}

One problem with the methodology used to design the system described in chapter 3 was that it attempted to strictly follow a series of initial specifications without considering their impact on performance; however, EHW allows designs to be flexible in their implementation. By moving to a methodology in which the specifications adapt to the ease of implementation, a more efficient design can be achieved. This may result in a lower resource usage and a higher maximum frequency. Additionally, having a simple design means that parts of it may be easily adapted to new purposes, thus making the system more versatile and reusable on new projects.

\footnotetext{
${ }^{19} \mathrm{~A}$ version of the SA with a clock enable will be discussed in $\S 5.4$.
} 


\section{Dealing with image borders}

A common issue of window-based image filters is what to do near the borders. For example, on a filter based on a $3 \times 3$ pixel window, each pixel is based on the surrounding ones. However, pixels on the top and bottom rows and the leftmost and rightmost columns are not completely surrounded by other pixels.

There are several approaches to tackle this problem. One of them is to add extra pixels on the image borders (typically by extending the last row/column). Another one is to simply discard these pixels, making the output image smaller than the input one.

The latter approach had been used in previous realizations of the system. However, it has some problems:

- The output image is smaller than the input one. This complicates the usage of multiple cascaded filters, as the border would need to be regenerated in each stage.

- There has to be a state machine that masks the output of the systolic array so that it is ignored for two clock cycles whenever the output reaches a border (for both the storage of the output image and the computation of the SAE). This complicates the hardware implementation, which requires additional logic and may reduce the maximum operating frequency.

In order to simplify the system, it was decided to simply ignore the fact that the image data is not continuous. Thus, as seen in Figure 5-28, a pixel on the border at either side of the image will be filtered as if a displaced copy of the leftmost/rightmost column were attached to the right/left side of the image. Therefore, from an implementation point of view, the sides of the image are not a special case, thus simplifying the control of the system. The top and bottom of the images are completed with zeros.

This border will not only be included as part of the filter output, but also in the computation of the SAE. Doing this implies that the filter will be partially trained to operate correctly even on the border, which could involve a larger filter complexity than if the border were just ignored. Nevertheless, the contribution of this border to the overall SAE of the image is small, and it was experimentally observed that the system was still able to evolve satisfactorily. 

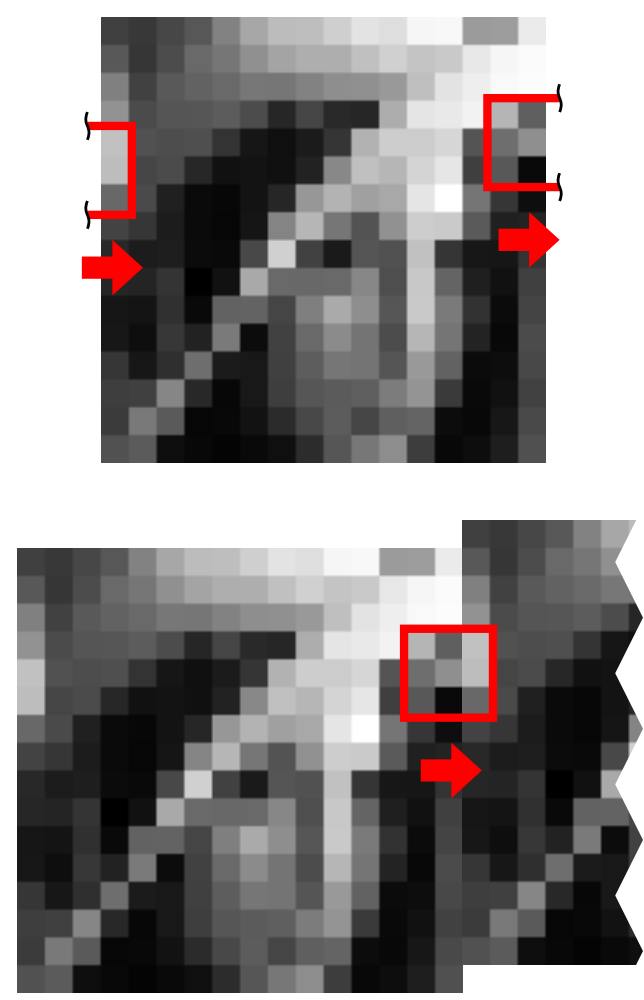

Figure 5-28: Window behavior at image border. When the window reaches the end of a line, it splits and continues on the next line (top). This is equivalent to extending the right side of the image with columns from the left side (bottom).

Original contribution 5-11 The choice of removing the special treatment of the image border, which simplifies the control. Additionally, this makes the output have the same size as the input, which simplifies the application of cascaded operations; this could be useful in datafloworiented applications as will be proposed in $\$ 5.4$.

\section{Impact of pipelining the SA inputs and outputs}

Registering the output of all PEs in the SA creates a pipelined architecture which allows operating at high frequencies-since each PE has a whole clock cycle to perform an operation-while keeping the throughput at 1 pixel per clock cycle.

This way of pipelining the SA ensures that data will propagate from PE to PE without mixing with data from other clock cycles. Therefore, the array output is identical to what would be obtained if there were no registers at all, but with a delay of a few clock cycles. 
The exception to this is the SA input. As can be seen in Figure 5-29, if the primary input is fed directly to the PEs, data will enter the SA on different "time zones", causing the output to be a result of input windows fed at different clock cycles. In other words, the effective input will not be a $3 \times 3$ pixel window as depicted, but will stretch horizontally.
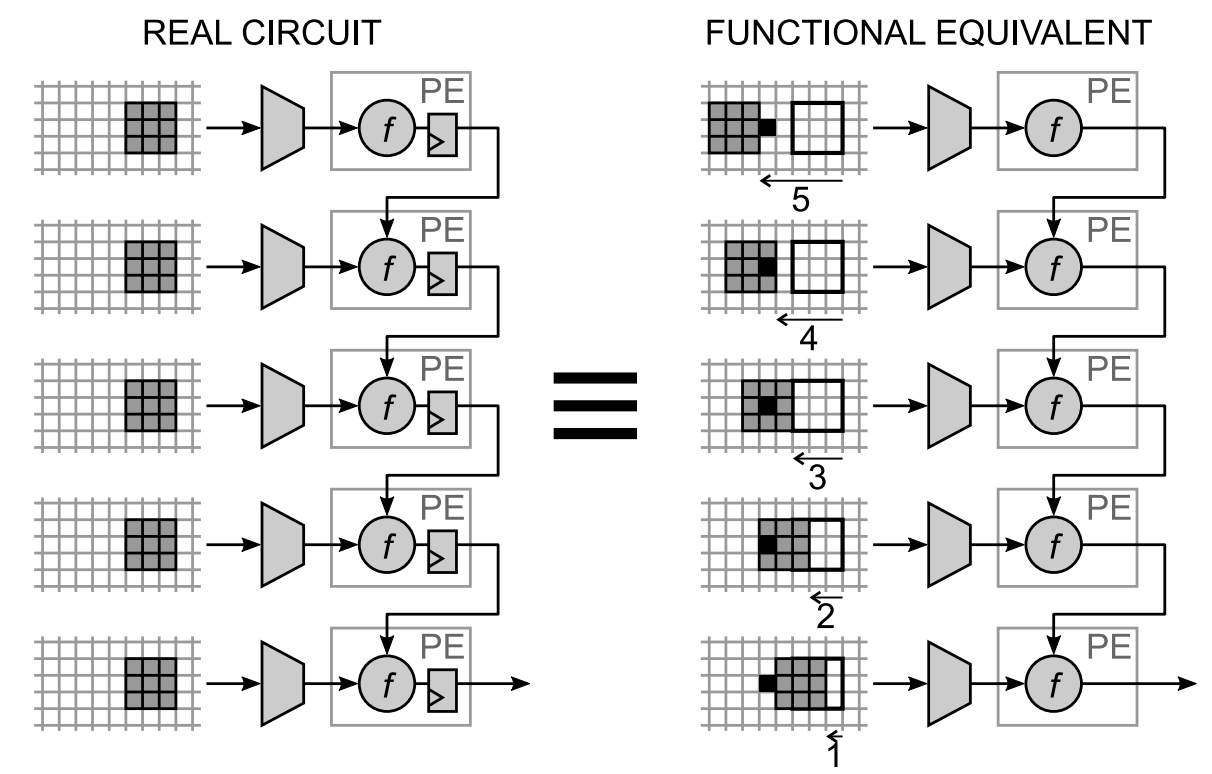

Figure 5-29: Left: $5 \times 1$ SA with inputs directly connected to the PEs, each of which registers its output. Right: this delay is equivalent to shifting the input window one pixel per clock cycle to the left; as a result, each effective input window is positioned differently with respect to the pixel marked in black.

This is a known peculiarity of the SA architecture, and can be easily solved by adding extra registers at the inputs and outputs, as it was shown in $\$ 5.1 .3$ (specifically, in Figure 5-10). These registers are often used in the literature [McCanny'82, Kung'84], also for other topologies such as CGP [Sekanina'03].

Nevertheless, having a stretched input window should not necessarily be a disadvantage. In fact, having a larger pixel sample could improve the quality of the filter. For this reason, previous work [Salvador'11a, Salvador'13, Gallego'13c, Mora'13b] neglected these registers and connected the SA inputs directly, obtaining satisfactory results.

However, this simplification presents several disadvantages:

- It makes the array latency uncertain and unpredictable, since it will be a combination of multiple latencies, each with a different impact on the result depending on the SA configuration. In [Salvador'13], this is 
solved by comparing the output with differently shifted versions of the reference by using multiple comparators-with the extra resource usage this involves-and reporting the best one.

- It makes certain inputs take a window that is too far from the "pixel of interest" (marked in black in Figure 5-29) rather than centered around it, which may make those inputs less useful. This effect can be seen as an advantage since it increases the effective window size, but, as Figure 5-30 shows, it actually results in a worse performance.

- It makes the system harder to characterize and simulate.

The effect of not pipelining the input is larger the bigger the array is, since the variability of the latency grows with the size of the array. Figure 5-30 shows the result of evolving arrays of size $4 \times 4$ and $8 \times 8$ without and with pipelining. As can be seen, the effect of pipelining the inputs is small for a $4 \times 4$ array (as used in [Salvador' 11a]), but for an $8 \times 8$ array it noticeably improves the results.
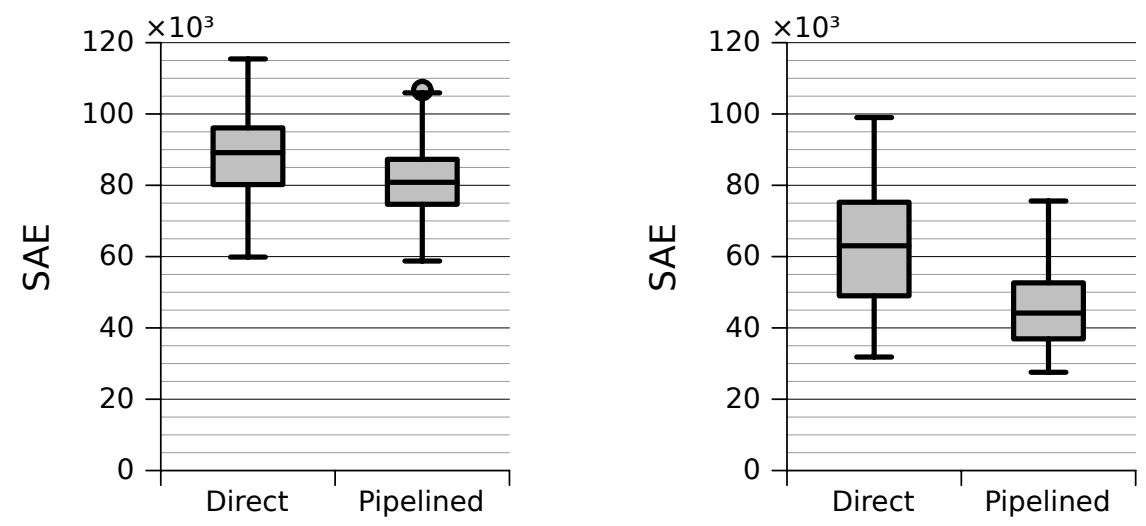

Figure 5-30: Fitness (SAE, lower is better) obtained after evolving $4 \times 4$ (left) and $8 \times 8$ (right) SAs, showing the effect of pipelining the inputs and outputs instead of connecting them directly.

In conclusion, introducing registers at the input of the SA for pipelining purposes greatly improves the performance of the system for big SA sizes, and removes the uncertainty on the SA latency, thus removing the need for multiple comparators. 
Original contribution 5-12 The analysis of the effect of introducing a proper pipelining of input and output over the evolution results. This pipelining also simplifies the design, removing the need for redundant comparators. Notice that such pipelining is not unique from this work, since early SA implementations already suggest its use [McCanny'82, Kung'84].

\section{Optimizing the fitness computing unit}

The fitness (SAE) computing unit performs a series of algebraic operations which may be excessively complex to perform on a single clock cycle at high frequencies.

An easy way to improve the timing behavior of this is to pipeline the unit so that error and accumulation are computed in different clock cycles. However, this may not be enough for very high operating frequencies.

Therefore, this unit has been divided into a parameterized number of modules, so that several pixels can be compared simultaneously (Figure 5-31). Each of the modules calculates a partial sum of absolute errors, and when the whole image has been received, all the partial sums are added together to form the result. This allows reducing the clock frequency of this unit while maintaining its throughput.

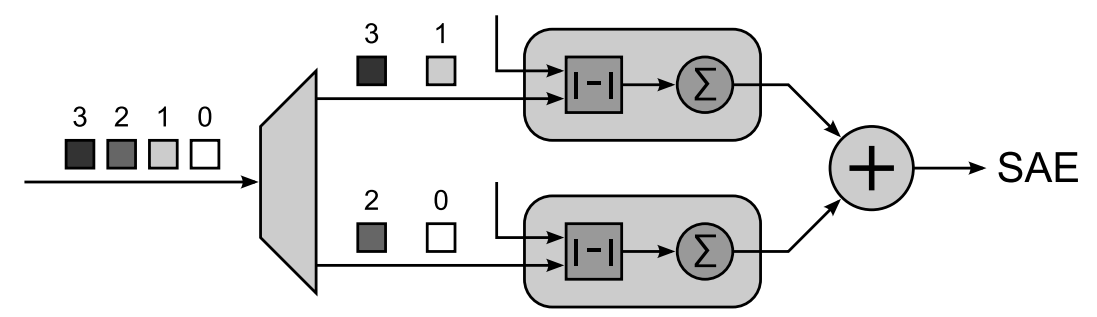

Figure 5-31: Parallelized fitness computing unit with a parallelization level of 2

This approach gives the designer freedom to choose an appropriate level of parallelization that compromises operating frequency and resource usage.

Original contribution 5-13 The parallelization of the fitness computing unit in order to obtain a configurable tradeoff between performance and resource usage. 


\subsubsection{Accelerating the evolution using multiple arrays in parallel}

As it has been seen, the filtering speed can be greatly improved by optimizing the system and SA implementation, reducing the time needed to evolve. However, no matter how good the design is, there is still a physical limitation to the maximum frequency achievable: the maximum switching frequency of the FPGA. Specifically, the FFs in the Virtex-5 FPGA in use have a maximum switching frequency of $600 \mathrm{MHz}$ [Xilinx-DS202].

One way to further accelerate the training stage of EHW while respecting this limit is to evaluate multiple solutions in parallel. This approach is not new; for example, it is used in [Dobai'14], where a CGP implementation originally able to evaluate 8700 candidate solutions per second uses 6 arrays in parallel to increase this figure to $15000 .^{20}$ Nevertheless, this has been considered a rather beneficial methodology that should be adopted into the design.

A very simple way to achieve this parallelization would be to just put several copies of the SA-based hardware module into the system, each with its own SA, fitness computing unit, memories, and control state machine. However, this would be very resource-consuming.

Since this is only going to be used during the training stage, which uses the same training input for all the candidate evaluations and does not use the training output at all, the system could use a single common set of input and reference memories and their corresponding control logic. Also, since fitness is computed in hardware, the output memories are not necessary during the training stage; the system only needs to store the output of a single filter for its operation after the training stage. Therefore, the only parts of the design that would need to be replicated are the systolic array and the fitness computing unit.

It should be noted that the reconfiguration process cannot be parallelized, and therefore it will be a bottleneck in the evolution speed, which will not grow proportionally to the number of arrays. The effect of having multiple arrays in parallel on the evolution time, together with an analysis of the optimum number of arrays and an EA that takes advantage of this parallelization, will be discussed in $\$ 6.2$.

On a final note, it is worth mentioning that one of the advantages of intrinsic EHW is that fault recovery is intrinsic to the evolution. However, if multiple arrays-assumed to be identical—are used in parallel, a fault in one of the

\footnotetext{
${ }^{20}$ This figure does not account for the further reconfiguration optimizations performed in [Dobai'14], with which the authors reach a total of 30000 evaluations per second.
} 
arrays will not be reflected in the output of another, so the evolution may not generate a solution resilient to this fault. Therefore, if a fault tolerant system is desired, this kind of parallelization should not be used directly; nevertheless, it would be possible to develop a new evolution methodology aware of faults across multiple arrays, but this has been left out of the scope of this thesis.

\subsubsection{Analysis of the solution and experimental results}

Once the proposed solution has been implemented, it has been characterized in order to determine its resource usage and performance. The results of this characterization are shown below, together with a summary of the parameterization of the system.

\section{Summary of configurable parameters}

By following a design methodology that heavily relies on parameters rather than hard-coding these values, a very versatile and configurable system has been obtained. Specifically, the following parameters have been implemented as VHDL generics:

- Number of SAs.

- Height and width of the SAs.

- Position of each of the SAs within the FPGA.

- Height and width of the sliding window up to $5 \times 5$ pixels.

- Height and width of the images (input, output, reference). ${ }^{21}$

- Parallelization level of the fitness computing unit.

- Whether or not PEs use the two-output scheme described in $§ 5.2 .4$.

- PE data width.

The possibility of using data widths larger than 8 bits (e.g., 16 or 32 bits) would allow using this filter for processing non-image data with higher precision requirements, such as analog signals coming from an EEG sensor. However, this feature has not been tested; evaluation of the benefits of its exploitation has been left as a candidate for future work.

\footnotetext{
${ }^{21}$ This would allow reducing the size of the training images for an evolution speedup; however this BRAM-based system is not suited for very large images such as $640 \times 480$ as these would not fit in the available BRAM.
} 


\section{Resource usage}

In order to characterize the resource usage of the EHW-based filtering system, a complete design has been implemented under Xilinx ISE. This design contains a MicroBlaze-based processing system, a reconfiguration engine, and two versions of the EHW peripheral, one with a single $8 \times 8$ PE SA and another with 12 of them.

The resource usage report is detailed in Table 5-8.

\begin{tabular}{lrrr} 
Part & LUT & FF & BRAM \\
\hline SA-based filter (1 SA) & $\mathbf{2 6 1 1}$ & $\mathbf{2 1 8 4}$ & $\mathbf{1 2}$ \\
SA & 1344 & 1408 & - \\
Padding LUTs (see $§ 5.1 .4)$ & 256 & - & - \\
Comparator & 427 & 240 & - \\
Rest of logic and memory & 457 & 352 & 12 \\
MicroBlaze interface & 127 & 184 & - \\
\hline SA-based filter (12 SAs) & $\mathbf{2 5 9 2 2}$ & $\mathbf{2 1 3 8 2}$ & $\mathbf{1 2}$ \\
SA (each) & 1344 & 1408 & - \\
Padding LUTs (each) & 256 & - & - \\
Comparator (each) & 427 & 240 & - \\
Rest of logic and memory & 1047 & 1012 & 12 \\
MicroBlaze interface & 551 & 594 & - \\
\hline Reconfiguration engine & $\mathbf{6 4 6}$ & $\mathbf{4 6 2}$ & $\mathbf{1}$ \\
\hline Processing system & $\mathbf{1 8 0 3}$ & $\mathbf{1 4 7 6}$ & $\mathbf{1 6}$ \\
\hline \hline Available in Virtex-5 LX110T & 69120 & 69120 & 148
\end{tabular}

Table 5-8: Resource usage of the EHW system

As it can be seen, the resource usage (LUTs and FFs) of the control logic and MicroBlaze interface represents roughly a $23 \%$ of the total amount used by the SA-based filter with a single SA, so when this is scaled to 12 SAs, a $20 \%$ of resources is saved with respect to implementing 12 separate filters, as anticipated in $\$ 5.3 .4$. This saving becomes most obvious in the BRAM usage, since requiring 12 times as much BRAM would have exhausted all the BRAM available in the FPGA.

Additionally, it can be observed that the resource usage required by the reconfiguration engine is small compared to that of the SA, which proves that using DPR can be beneficial over other solutions such as VRC even despite of this resource overhead.

A complete processing system with a MicroBlaze, reconfiguration engine, and a single $8 \times 8$ SA would only use $7 \%$ of the LUT and FF resources available 
in the target FPGA, leaving a lot of room to implement additional hardware modules. An implementation using 12 SAs of the same size ${ }^{22}$ would still fit in that FPGA, using $41 \%$ of the resources.

\section{Experimental evaluation of the maximum frequency}

All individual parts of the filtering system have been measured to have a maximum operating frequency of $400 \mathrm{MHz}$ or more according to the vendor's timing report tool, except the SA itself which, as it was said in $\$ 5.1 .6$, reported a maximum operating frequency of $350 \mathrm{MHz}$. Nevertheless, this is a rather conservative report based on the worst-case behavior of the system. In order to obtain more realistic values, the system was overclocked to the following frequencies:

- Systolic array frequency: $400 \mathrm{MHz}$

- BRAM frequency: $100 \mathrm{MHz}$

- Comparator frequency: $100 \mathrm{MHz}$ (comparing 4 pixels in parallel)

- Reconfiguration engine frequency: $200 \mathrm{MHz}$

Despite the overclocking, the system worked perfectly, presenting no errors in the evolution and obtaining always the same result as the simulated model.

Speeds of 450 and up to $500 \mathrm{MHz}$ were also tested, but some errors were observed at those speeds under certain conditions, e.g. when implementing multiple arrays in parallel or combining the system with other modules such as external SDRAM controller and video output. ${ }^{23}$

Nevertheless, even if the intermediate results were not absolutely correct at $500 \mathrm{MHz}$, they were stable enough for the EA to still obtain good results. Therefore, it would be possible to overclock the system during the evolution in order to accelerate it, and then go to nominal speeds once the training has finished. However, it was decided to keep the system at a predictable behavior, thus fixing the speed at a conservative value of $400 \mathrm{MHz}$.

With this operating frequency, the array is able to process 400 million pixels per second, and a $128 \times 128$ pixel grayscale image gets filtered in $42 \mu \mathrm{s}$.

\footnotetext{
${ }^{22}$ In $\S 6.2$ it will be seen that using 12 SAs in parallel maximizes the speed of the EA presented in said section.

${ }^{23}$ This is partly due to the fact that the system works better if all clocks are generated by the same PLL, which restricts the frequencies that can be obtained, since the external DDR2 SDRAM requires a clock of exactly $200 \mathrm{MHz}$. This makes a clock of $450 \mathrm{MHz}$ hard to obtain if the external RAM is used. A clock of $500 \mathrm{MHz}$ is easy to obtain but often presented timing problems.
} 


\section{Evolution speed}

Overall, the current implementation requires $53.8 \mu$ s to evaluate a single candidate solution. This includes the reconfiguration of the SA, the filtering of the training input and computation of the resulting fitness, and the time required by the microprocessor for the chromosome mutation and fitnessbased selection. These times are detailed in Table 5-9.

\begin{tabular}{lrr} 
Stage & \multicolumn{2}{c}{ Time $(\mu \mathrm{s})$} \\
\hline Mutation & 2.2 & $(4.1 \%)$ \\
Reconfiguration & 9.3 & $(17.3 \%)$ \\
Filtering & 42.0 & $(78.1 \%)$ \\
Selection & 0.3 & $(0.6 \%)$ \\
\hline Total & $\mathbf{5 3 . 8}$ &
\end{tabular}

Table 5-9: Evolution time breakdown in microseconds for a single evaluation

This makes the EA able to evaluate 19000 candidate solutions per second, a considerably higher figure than the previous 2600-5700 obtained in $\$ 3.2 .1$. Thus, a 100000 -generation evolution (with 8 children evaluated per generation) will take 43 seconds to complete.

This does not account for the possibility of using multiple SAs in parallel, which would result in a drastic reduction of the filtering time, which represents the biggest fraction of the total. This approach is to be exploited and characterized in $\$ 6.2$.

\subsubsection{Conclusions}

In this section, a highly optimized and parameterized architecture for the control of an SA for image filtering applications has been implemented. This has been achieved thanks to a design philosophy that emphasizes modularity and simplicity of design.

This implementation serves as a demonstration of the discussed DPR design methodology, and also provides an evaluation platform for different experimental analyses in order to characterize different EAs and design considerations. In addition, the developed system is a useful asset that could be used in other projects as an accelerated image processing peripheral. 


\subsection{MOVING TO A DATAFLOW PARADIGM}

The previous section described the efforts made into dividing the BRAM-based evolvable image filtering system into separate modules in order to promote its generalizability and adaptation to potential new tasks. In principle, this would facilitate the task of adapting the system to be used in applications such as a real time video processing platform.

However, having an image processing system that relies on memories to store the input and output images presents a disadvantage: although this approach is suited for training an evolutionary system, which needs to filter the same image over and over, using it as a real time video processing system requires writing the input image to this memory, filtering it, and reading the result from memory. This slows down the process considerably, since memory transactions between microprocessor and peripherals are typically slow.

In addition, the current system has a fixed image size of $128 \times 128$ pixels. This is not realistic for real time video processing, since common video formats typically range from $640 \times 480$ (VGA) to $1920 \times 1080$ (Full HD).

Filtering a $640 \times 480$ pixel image would require input and output image memories with sizes of $300 \mathrm{~KB}$ each $(600 \mathrm{~KB}$ in total); however, the amount of BRAM memory available in a Virtex-5 LX110T FPGA is only $592 \mathrm{~KB}$ [Xilinx-DS100]. ${ }^{24}$ This could be solved by using a smaller memory as a circular buffer [González'17]; however, a simpler solution would be to get rid of these intermediate memories.

Another problem of the system described in $\$ 5.3$ is that, although it has been divided into independent modules, creating a derived system that changes the flow of data between these modules (as is done in [Gallego'12], for example) involves changing the HDL code of the system. A more beneficial design methodology would be to replace this "monolithic" IP approach with a collection of smaller IPs that can be interconnected in a simple way.

In order to solve these problems, it was seen necessary to adopt a dataflow paradigm, this is, a design methodology based on data streams where multiple blocks can be cascaded without needing to store intermediate results in memory. This would promote not only the design of complex systems with the building blocks developed in this thesis, but also the integration with other preexisting video processing blocks. For example, Xilinx provides a wide

\footnotetext{
${ }^{24}$ XC5VLX110T contains 5328 Kbit of BRAM [Xilinx-DS100, table 1], which corresponds to $592 \mathrm{~KB}$ of usable data (one byte $=8$ bits of data +1 bit used for error correction).
} 
range of video processing IP cores [Xilinx-PG231], as well as tools for designing custom ones [Neuendorffer'15]; the possibility of combining these IP cores with an EHW system could open the door to many interesting projects.

\subsubsection{Towards a dataflow-oriented paradigm}

The idea of adopting a dataflow-based approach arises as a generalization of some preliminary implementations of cascaded SA-based image filters [Gallego'13b, Gallego'14]. These works exploited scalability in the number of arrays by permitting multiple ways to interconnect individual SA-based modules (Figure 5-32).

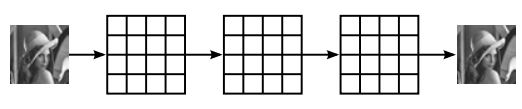

(a)

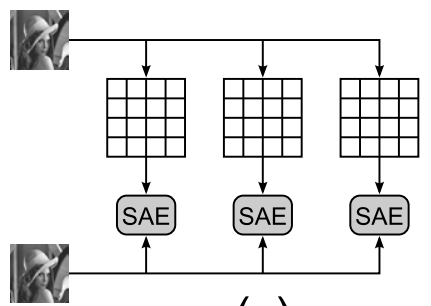

(c)

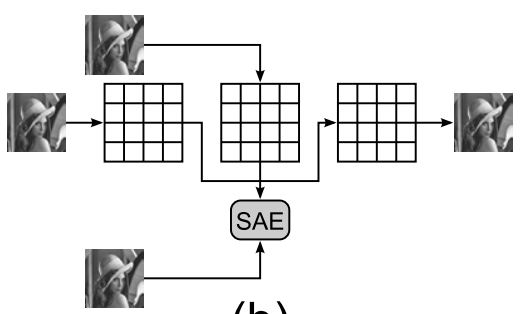

(b)

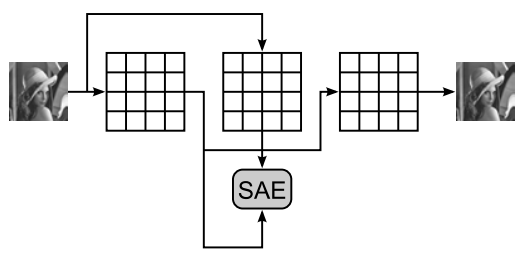

(d)

Figure 5-32: Different interconnection possibilities, as seen in [Gallego'14]: (a) cascaded, (b) bypass, (c) parallel, and (d) "learn by imitation".

In [Gallego'14], this was achieved by designing individual filtering stages with a wide range of interconnection possibilities provided by multiplexers, as shown in Figure 5-33. This not only permitted using the arrays in a cascaded manner - which is useful to implement multi-stage filters with better capabilities than a single one-, but also having three arrays in parallel-for increased throughput or redundancy—, training one filter while the others are processing data-e.g., to fix a fault in the filter-, or using the comparator module to compute the "quality gap" between input and output rather than the difference between output and reference (which will be used in $\S 7.1 .3$ as a way to monitor changes in the problem conditions). Furthermore, these stages were designed to be easily cascadable, with the long-term goal of making them 
dynamically reconfigurable, thus varying the number of arrays dynamically at run time.

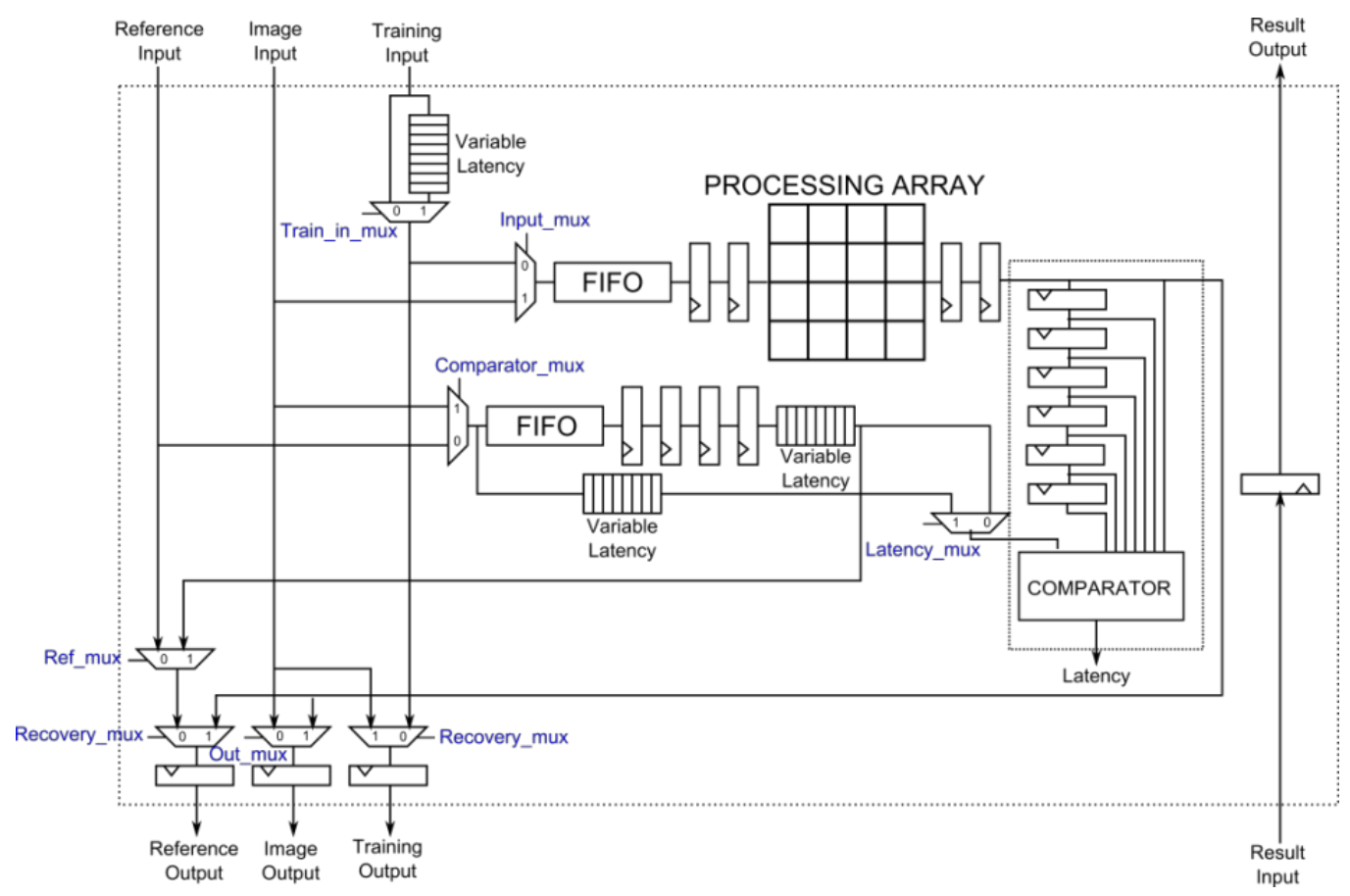

Figure 5-33: Diagram of a single SA-based processing module in [Gallego'14]

However, this approach had a fundamental problem: it was highly specialized for a specific set of tasks, not permitting the implementation of arbitrary interconnection topologies outside of its scope without redesigning the system at HDL level.

This led to a reconsideration of the design, moving from a fixed interconnection scheme to a configurable one. This aims at providing a mechanism to implement arbitrary dataflow schemes. These schemes may be configured either statically at design time or dynamically at run time; the latter would be specially beneficial combined with DPR of modules. Figure 5-34 illustrates this through an example based on a Sobel filter (top), which may either be implemented in a cascaded mode with a static interconnection structure (middle) or use DPR of modules and dynamic interconnections in order to implement the filtering process in four steps (bottom); the latter approach requires using a few memory blocks to store intermediate results but would be useful when FPGA resources are scarce and the latency requirements are more relaxed. 

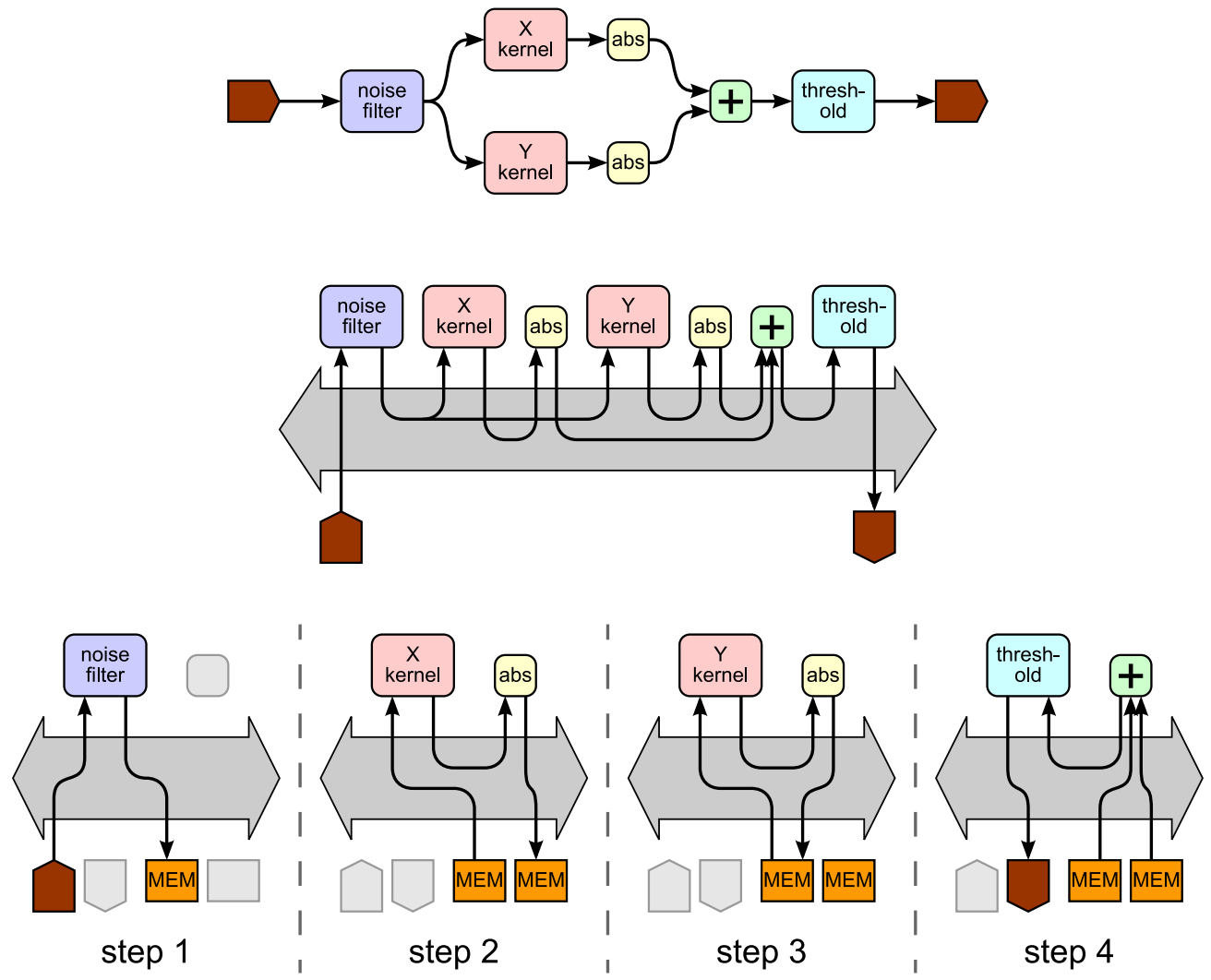

Figure 5-34: Implementation of a Sobel filter (top) using static (middle) and dynamic (bottom) approaches.

In $\S 5.3$, the design was split into modules, facilitating the task of designing new systems with some modifications to the HDL code. However, modern hardware design software such as the Vivado design suite tends to focus less on HDL and puts emphasis on a visual block-based design.

The dataflow-based approach presented here aims to provide the following enhancements to the design methodology:

- A design approach based in self-contained blocks that are directly reusable without requiring to rewrite HDL code.

- The ability to elaborate complex processing structures by interconnecting basic predefined blocks.

- The possibility to make these blocks dynamically reconfigurable in a future. 
Therefore, it becomes necessary to define a common interconnection mechanism for these blocks that is simple enough to implement, and to adapt the system modules to this mechanism.

Original contribution 5-14 The implementation of an evolvable filter based on an $S A$ as a dataflow-oriented video filter.

\subsubsection{Choosing an interconnection interface: AXI4-Stream}

The choice of an appropriate interconnection interface has been made following not only simplicity and versatility considerations, but also feasibility of adoption in future projects, in order to promote and simplify the use of the system developed here-as well as other systems developed using the same design methodology—within said projects.

Although designing a custom interface from scratch has been considered as a possibility, eventually it has been decided to adopt the preexisting AXI4Stream Video protocol, for reasons that will be detailed in this subsection.

\section{The future (and present) of Xilinx: Vivado Design Suite}

So far, this project has been developed under the Xilinx ISE Design Suite, a collection of simulation, synthesis, and other development tools which provides support for Xilinx FPGAs and CPLDs. However, this tool saw its last version in 2013, and is being phased out by Xilinx in favor of a new one: the Vivado Design Suite. Vivado aims to provide better integration between its different parts, and brings a design approach based on a visual placement and interconnection of IP cores (Figure 5-35).

Among other things, Vivado puts emphasis on replacing the previous heterogeneous range of buses (PLB, FSL, NPI, LL...) with a consolidated bus interface specification: ARM® AXI $^{\mathbf{T M}}$. This versatile interface is used in Vivado designs to configure peripheral registers, perform memory-mapped accesses, and transmit point-to-point streams of data [Xilinx-UG761].

Vivado provides a wide range of video processing IP cores [Xilinx-PG231], which use an AXI interface for the transmission of video streams. Additionally, the Vivado High Level Synthesis (HLS) application allows designing custom IPs with a wide support for video applications [Neuendorffer'15], also using this interface. 


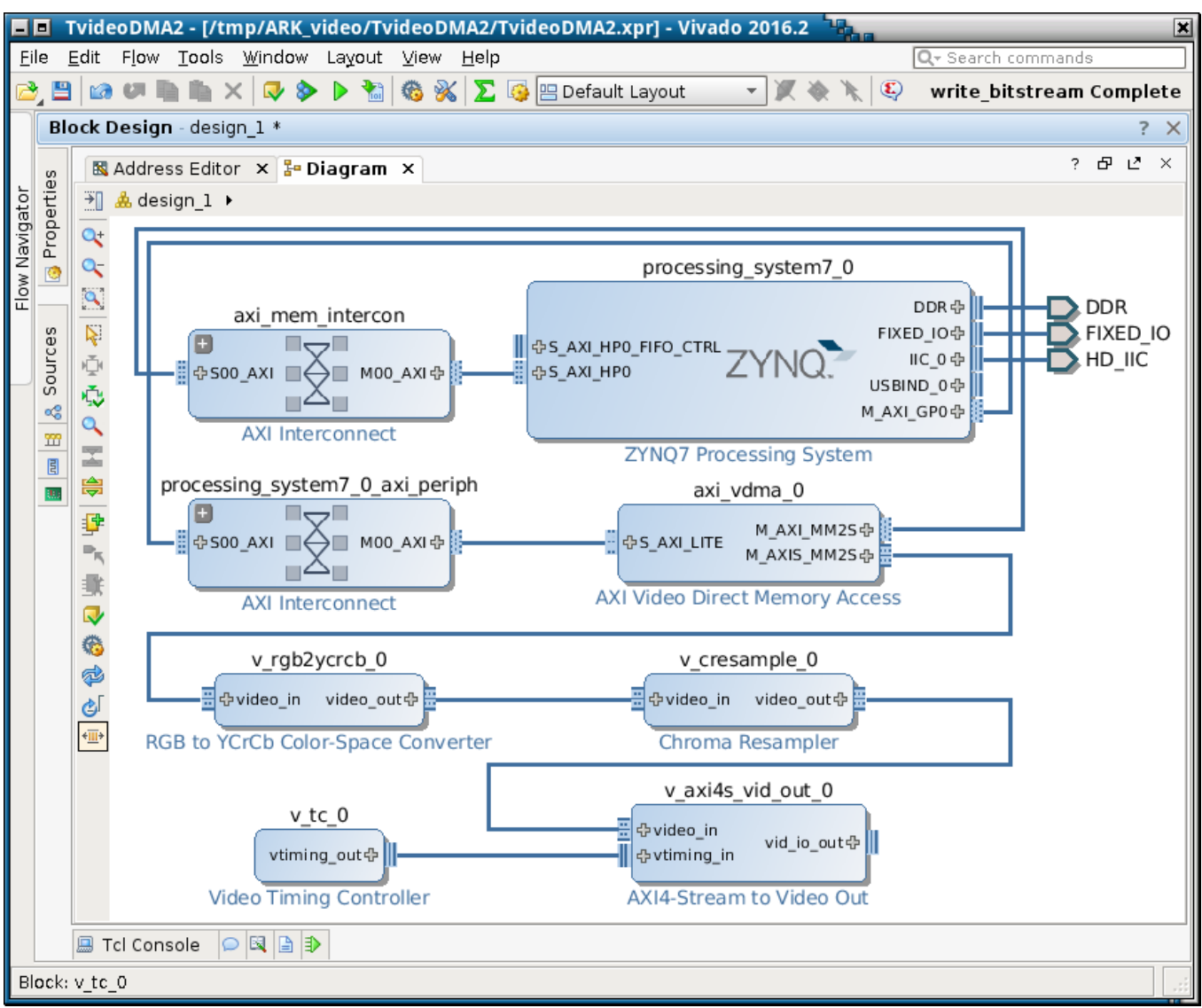

Figure 5-35: Screenshot of Vivado IP Integrator displaying a block design

Given the simplicity and ubiquity of the AXI interface, it has been decided to adopt it as the interconnection interface between blocks in the dataflow design approach. This will make these blocks able to be integrated with other preexisting IP cores provided by Vivado or by third parties, bolstering the use of this methodology in future projects.

\section{What is AXI?}

Advanced eXtensible Interface (AXI ${ }^{\mathrm{TM}}$ ) is a protocol specification developed by ARM ${ }^{\circledR}$ as part of the Advanced Microcontroller Bus Architecture (AMBA $\left.{ }^{\circledR}\right)$ specification [ARM-IHI0022E]. This protocol is intended for use in highbandwidth and low-latency designs.

In its fourth version, this protocol has three variants: AXI4, which provides a full featured address-based interface capable of burst transactions, intended as a memory interface; AXI4-Lite, which is a lightweight subset of AXI4 
for single word transactions, intended for register access; and AXI4-Stream [ARM-IHI0051A], which provides a FIFO-style point-to-point communication intended for unidirectional streams of data (such as video streams).

All these protocols are based on one or more communication channels featuring a synchronous valid-ready handshake (Figure 5-36): when the upstream module has available data, it outputs the data and asserts the valid signal; and when the downstream module is ready to accept a new data word, it asserts the ready signal. When both valid and ready are asserted, it is considered that the transaction has been made and each module sets up the valid or ready signal for the next clock cycle. This permits a throughput of one transaction per clock cycle with a small latency.

This handshake protocol is similar to a FIFO interface, except that instead of read and write signals that have an immediate effect, and therefore have to consider the state of full and empty signals during the same clock cycle, the ready and valid signals are not effective until the other one is, and thus can be set independently of the other module, leading to a synchronous controlling interface which is simpler to implement.

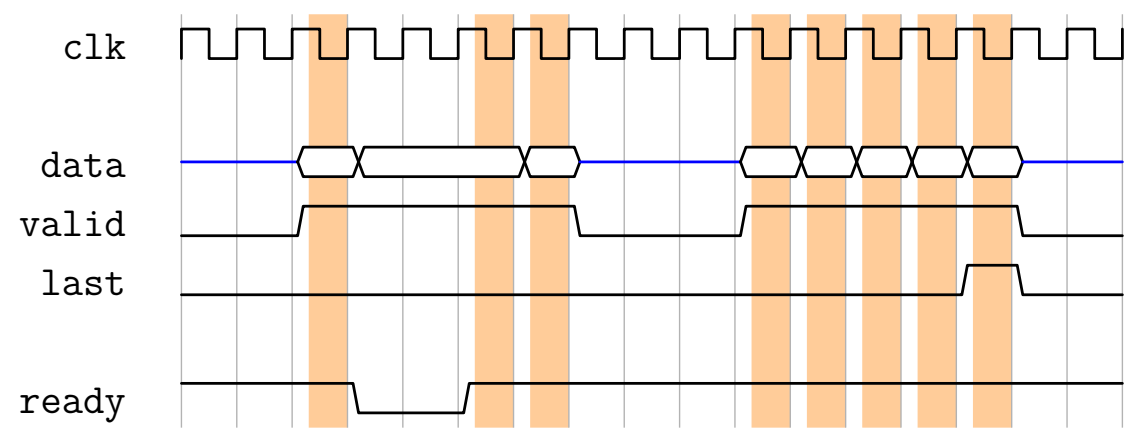

Figure 5-36: Valid-ready handshake. Transactions are shaded in orange.

In order to transmit video data, Xilinx defines its own AXI4-Stream Video protocol [Xilinx-UG934] as a specialized subset of AXI4-Stream with the following signals: DATA [8n-1:0], which transmits $n$-byte pixel value information; VALID and READY, which perform the handshake described before; EOL, which marks the last pixel on a line; and SOF, which indicates the first pixel of a frame. There is no signal to indicate the end of a frame; video processing cores are responsible of figuring this by counting the lines received after SOF has been asserted.

Figure 5-37 depicts an implementation of this signal interface for 8-bit grayscale video transmission as needed for the system at hand. As it can 
be seen, this interface requires only 12 one-bit nets, which may potentially simplify the task of making these interconnections dynamically reconfigurable with tools like DREAMS.

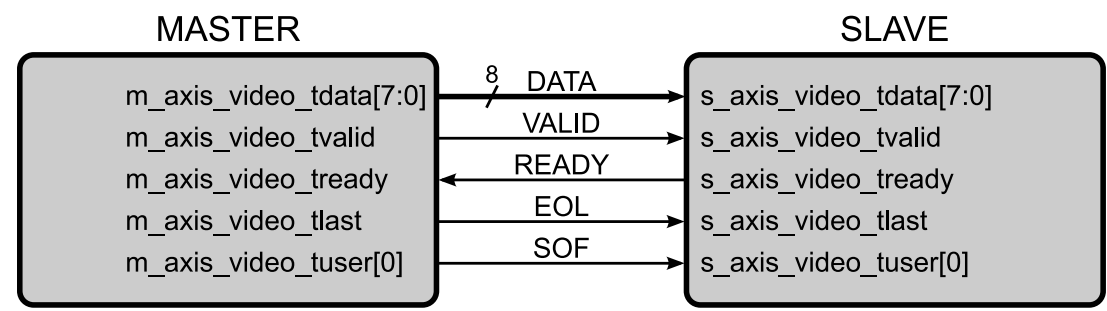

Figure 5-37: AXI4-Stream Video bus for an 8-bit video signal

\section{The AXI4-Stream Infrastructure IP Suite}

The primary advantage of adopting an AXI4-Stream Video interface is its wide support in Vivado and its associated IP availability. As it has been said, this interface is a subset of AXI4-Stream, and therefore it is directly supported by design methodologies targeting AXI4-Stream. Specifically, Vivado provides the AXI4-Stream Infrastructure IP Suite [Xilinx-PG085], which is an extensive collection of modular IP cores facilitating the interconnection of AXI4-Stream blocks and the routing of data streams.

This infrastructure provides several solutions to problems in the current design methodology that so far required to be addressed by developing custom HDL code. Some examples of these previous problems, as well as some potential requirements that may appear in a dataflow approach, are:

- Multiplexing streams of data. This is probably the most important feature provided by the AXI4-Stream Infrastructure for this project. In [Gallego'14], multiplexers were used in order to select the source of pixel data for different parts of the design. However, this addressed only a very specific use case and did not provide a general solution. In order to have a more generalized design methodology, streams can be multiplexed using the AXI4-Stream Switch, which permits selecting a stream from different sources, and routing this stream to the intended target, all while dealing with the valid and ready signals correctly.

- Adapting data streams to different clock frequencies. This was tackled in $\$ 5.3$ by using a custom implementation of a dual-clock FIFO, since the FIFO Generator tool provided by Xilinx ISE did not allow much 
customization and parameterization. However, the AXI4-Stream Clock Converter provides a more elegant solution which is less prone to implementation errors.

- Packing (and unpacking) multiple pixels into single words. This can be used for reading or writing multiple pixels in a single BRAM access, thus permitting different clock rates and potentially reducing energy consumption. This functionality is provided by the AXI4-Stream Data Width Converter.

- Forking a stream of data to multiple sinks. This is needed for example when sending the filtered image to both the output BRAM and the SAE computing unit. This was trivial to do in $\$ 5.3$ since it only required proper clocking, but on an AXI4-Stream system there needs to be proper handling of the valid-ready handshake for all the endpoints. This can be achieved using the AXI4-Stream Broadcaster, which allows "cloning" a data stream so that it can be sent to multiple targets simultaneously. This IP also allows having each target receive only a slice of each data word, which could be useful for example for splitting an RGB video signal into its three components to be processed separately.

- Combining multiple streams together. The AXI4-Stream Combiner is the counterpart of the previous module, and permits merging multiple data streams into a single one (for example, to combine several R, G, and B components into a single RGB signal). This could also be useful for example for implementing an image comparator, which requires pixels from the two sources to be available two by two.

- Accomodating data paths with different latencies. Having multiple data paths that fork and combine at different points could result in a deadlock if these paths have unmatching latencies. This can be overcome by using an AXI4-Stream Data FIFO on the path with lower latency so that it acts as a data buffer. These FIFOs may also be used when the latency of these paths is unpredictable.

The ease with which a dataflow-based system can be built using this AXI4Stream Infrastructure IP Suite is an additional reason to switch to Vivado, as it poses a great improvement in the design methodology.

Original contribution 5-15 The adoption of the AXI4-Stream Video protocol for the SA-based filter is a potentially useful contribution towards future applications, since it enables the integration of the $S A$ system with other video processing modules using a preexisting protocol. 


\subsubsection{Porting the system to the new paradigm}

Once the characteristics of the dataflow approach have been outlined, the system has been adapted to this paradigm. This subsection describes the challenges that this work has involved, motivating the different design decisions adopted to tackle them.

\section{Adapting the design to the 7 Series}

The first challenge encountered when porting the system to a Vivado-based dataflow solution is the fact that Vivado does not support FPGAs earlier than the 7 Series [Xilinx'12]. Therefore, the Virtex-5 LX110T FPGA that has been used so far in this work was abandoned ${ }^{25}$ for the dataflow paradigm in favor of Zynq7000-based devices, such as the XC7Z020 all-programmable $S^{26} C^{26}$ present in development boards such as Zedboard and PYNQ. The Zynq platform has been described by some authors as an excellent platform for EHW design [Dobai'13].

As it was already stated in $\$ 5.1 .1$, the internal architecture of 7 Series devices shares many similarities with the Virtex-5 family, reducing the amount of work required to port the system (both the SA-based filter and the reconfiguration engine) to the 7 Series.

Maybe the most notable difference between Virtex-5 and 7 Series implementations is how the two stages of a PE described in $\$ 5.1 .2$ are arranged: while in Virtex-5 these were placed side by side occupying two CLBs (as seen in $\$ 5.1 .4$ ), for the 7 Series version it has been decided to arrange them vertically, thus making a PE span four CLBs in height and half a CLB in width (Figure 5-38). This is motivated by the fact that 7 Series FPGAs have taller CLB columns, containing 50 CLBs rather than 20; and thus a taller and narrower PE geometry will adapt better to this FPGA and make a better use of resources. This change has required some minor modifications on the HDL code for the SA and reconfiguration engine, as well as on the software procedures controlling the reconfiguration.

Eventually, a BRAM-based reconfigurable SA subsystem for the 7 Series was obtained, using AXI4 and AXI4-Lite interfaces for memory and register access. This system does not yet fulfill the goal of following a dataflow

\footnotetext{
${ }^{25}$ Given the large amount of work and results based on the Virtex-5 LX110T obtained in this thesis, this FPGA will continue being used in ongoing chapters, specifically in chapter 6 and sections 7.1 and 7.2. Nevertheless, given its lack of support in newer tools, it is planned to eventually abandon this platform completely.

${ }^{26}$ Xilinx all-programmable SoCs integrate an FPGA, one or more ARM cores, on-chip memory, and other components such as math coprocessors on a single IC [Xilinx-UG585].
} 
VIRTEX-5

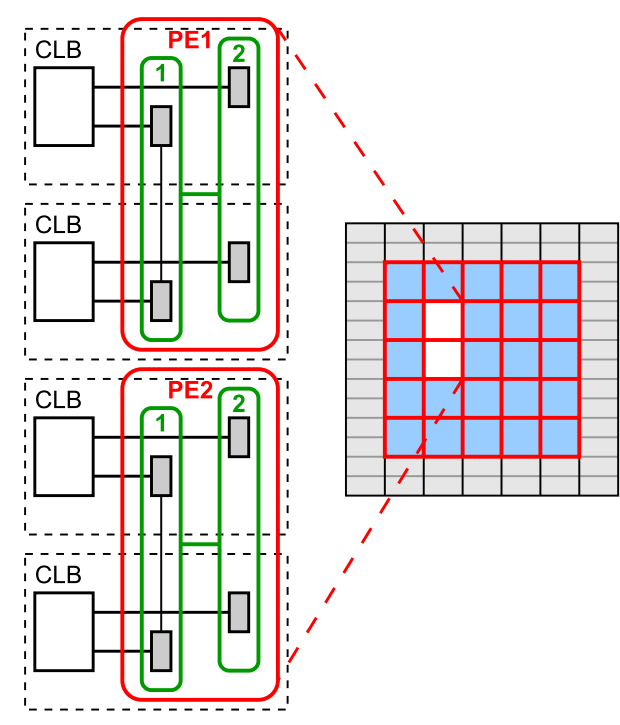

7 SERIES

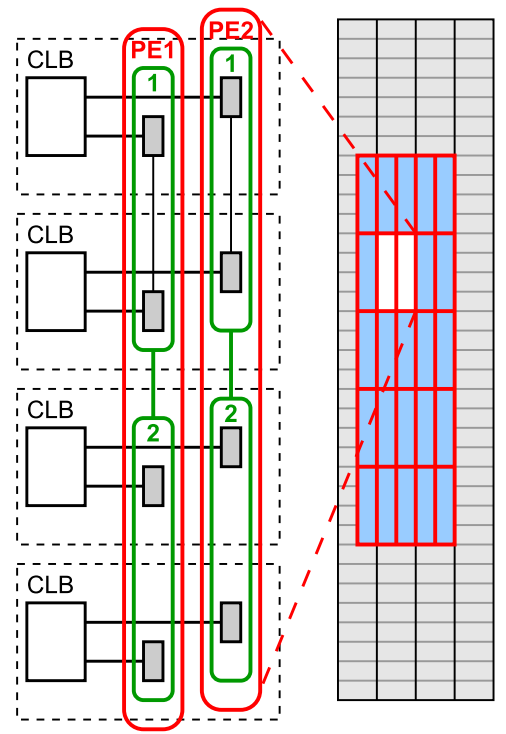

Figure 5-38: Arrangement of the two stages (green) of a PE (red) in Virtex-5 and 7 Series implementations, showing how it affects the shape of an SA (blue) and its occupation of CLB columns (gray), which are larger in 7 Series FPGAs.

paradigm; however, this porting effort has been useful for verifying that the 7 Series implementation of the reconfigurable SA is correct, and permits a direct implementation of the old system on the new technology.

\section{Making the SA compatible with a dataflow approach}

In order to adapt the BRAM-based image processing IP core to the dataflow paradigm, an alternative core was developed which replaces the image memories and their corresponding AXI4 interfaces with a pair of AXI4Stream Video interfaces. In order to keep the system simple and modular, this IP lacks a fitness computing unit, limiting to the SA itself, a window generator, and a simple state machine to control the AXI4-Stream Video interfaces-if fitness computing is needed, it may be implemented as a separate block.

A key difference between the dataflow approach and the previous memorybased one is that there is no guarantee of the availability of data at the input nor the possibility of transmitting processed data to the output, due to the presence of the valid-ready handshake described in $\$ 5.4 .2$. Previously, it was assumed that upstream and downstream modules would always be able to ensure a throughput of one pixel per clock cycle at the SA frequency, and 
the system was designed in such a way that this constraint would be fulfilled. However, the presence of valid and ready signals on an AXI4-Stream interface present the possibility of upstream data momentarily ceasing to be available or downstream modules becoming unable to process any further data, both of which escape the control of the image filtering system.

This is an improvement over the previous approach as it makes no assumptions on the throughput of the SA with respect to upstream and downstream modules, making the system more versatile in terms of frequency and interoperability, but requires some care to be taken on the implementation in order to prevent disrupting the correct pipelining of the system. This issue has been addressed by adding a clock enable (CE) signal to the SA by using the CE input of its FFs (Figure 5-39). This way, the whole SA can be halted when either the upstream valid or the downstream ready are not asserted, without requiring any major change in the SA design except for the addition of an extra control signal.

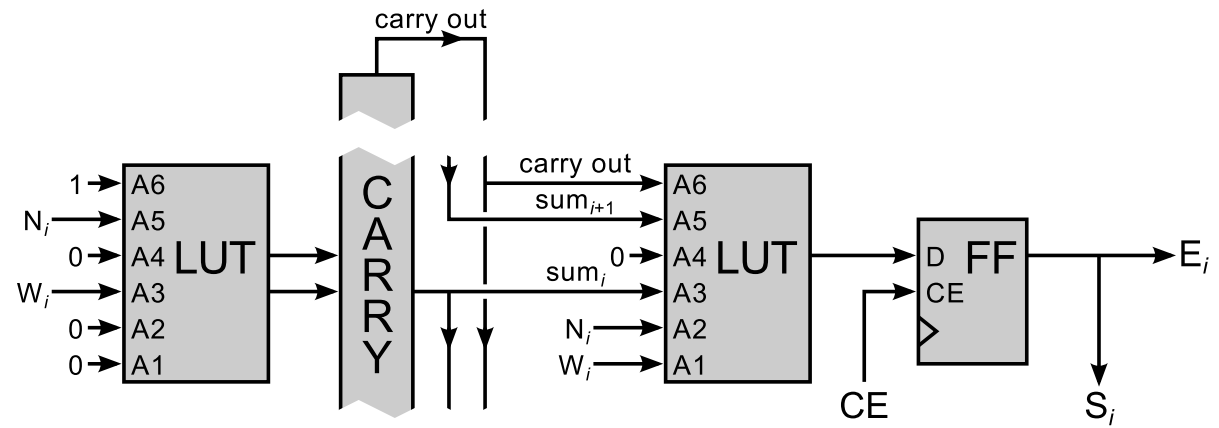

Figure 5-39: Fragment of a dataflow-oriented PE. The design is similar to the one in Figure 5-6, with the addition of the $\mathrm{CE}$ input on the FF, as well as some minor changes in the LUT pin assignment to suit the requirements of 7 Series as discussed in $§ 5.1 .1$.

Aside from the use of the CE inputs on its FFs, the resulting SA is identical in its low-level architecture to the BRAM-based one, and therefore its LUTs can be configured with the same mechanism: their partial bitstreams are compatible. This is an important trait as it permits the use of a PBS obtained for a BRAM-based evolvable SA system to be used directly on its dataflow-based counterpart.

In addition to adapting the system to the AXI4-Stream interface, it is important to make it able to be configured for different frame sizes at run time, in order to enable its use within video systems supporting multiple resolutions. This has been achieved by replacing the previous window generator, implemented as a series of fixed-length shift registers, with one that 
uses a BRAM-based circular buffer (Figure 5-40), as it is done in [González'17]. The choice of a BRAM-based delay line over a shift register-based one allows for greater depths without excessive resource usage, thus allowing rather wide image formats (for example, a $3 \times 3$ window generator for a $1920 \mathrm{px}$ wide frame would use a single 36 Kbit BRAM block).

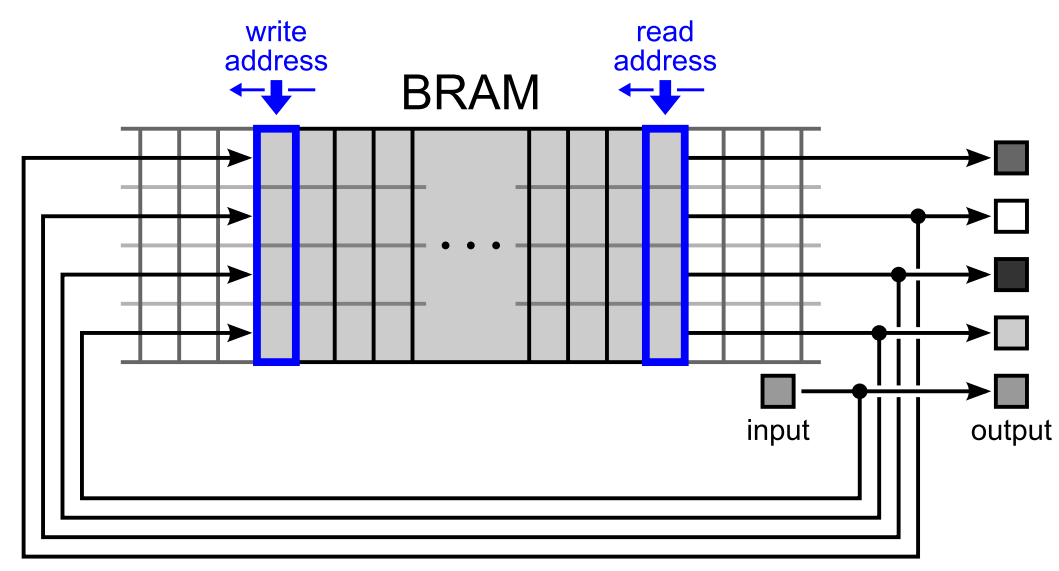

Figure 5-40: Variable width $5 \times 1 \mathrm{px}$ window generator implemented as a circular buffer using a BRAM with 32-bit (4 px) words. Different frame widths are handled by changing the separation between read and write addresses.

\section{Comparison of BRAM-based and stream-based approaches}

Although both the BRAM-based and the stream-oriented realizations develop a common task, both have advantages and disadvantages and are therefore better suited for different use cases.

In particular, the BRAM-based approach performs well in the evolution stage, as it only needs to read and filter the same BRAM data repeatedly. In addition, its current implementation permits the evaluation of multiple solutions in parallel ( $\$ 5.3 .4)$ in order to accelerate the evolution. However, this particular implementation is designed for small fixed-size input frames, making it unsuited for realistic video applications that have to deal with large frame sizes (unless some solution such as dividing the image into several $128 \times 128$ px blocks to be processed separately is applied, with the consequent software overhead this would imply). Additionally, copying the whole image to BRAM, waiting for the whole image to be processed, and copying it back from BRAM entails a large latency and substantially decreases the system throughput, making the BRAM-based approach unsuited for typical real time applications. 
A derived BRAM-based solution that allows filtering arbitrarily large frames of variable size is described in [González'17]. This implementation has the advantage of using the same module for evolution and real-time operation; however, the latter requires that the microprocessor be constantly writing image fragments to the IP core BRAM and reading the processed ones. This prevents the microprocessor from developing other tasks during that time, and causes the filtering to have a large latency (although not as great as if the whole frame had to be copied).

On the other hand, the stream-oriented approach has a very low latency (of only a couple of image lines), and permits arbitrarily large frame sizes as only a few lines need to be stored in BRAM rather than the whole frame. However, using this system for an evolution stage would require the microprocessor to be continuously issuing streams of image data, which may be less efficient than the BRAM-based approach in which the module only needs to receive a "start" instruction to initiate the image filtering. In addition, implementing a whole evolvable system based on the stream-oriented SA would imply developing a stream-oriented SAE computing unit, which has been deemed out of the scope of the work covered by this thesis (although it is a candidate for future work).

Therefore, the stream-oriented approach is ideal for real time applications but unsuited for the evolution stage, whereas the BRAM-based approach is ideal for the evolution stage but suboptimal for real time applications. Therefore, none of the two modules is suited for an evolvable real time video processing system.

The solution to this dilemma is to use both modules on the system: this way, while the BRAM-based subsystem is used to find the optimal solution, the stream-oriented SA system performs the real-time video filtering. These two modules may work in parallel, with the stream-oriented module working with the best solution available while the BRAM-based subsystem searches for better solutions, in a similar manner to what was suggested in $\$$ 3.3.5.

This approach may seem wasteful, but it provides a possibility of performing evolution and filtering at full capacity simultaneously. Nevertheless, future research lines may consider the possibility of implementing a single block that combines both functionalities using a single SA with multiplexed inputs and outputs in order to save resources, or implementing the SAE computing unit as well as BRAM read and write modules as separate AXI4-Stream Video modules, thus allowing the implementation of the original BRAM-based design through the use of basic interconnectable modules, which may be multiplexed using the appropriate utility from the AXI4-Stream Infrastructure. 
It should be noted that having two separate arrays for evolution and realtime operation means that the evolution is no longer intrinsic, and thus the fault tolerance of this system is lost, which further motivates the development of the aforementioned dual functionality SA.

Original contribution 5-16 The dual use of BRAM-based and streambased versions of the SA filter (the former merely as a hardware accelerator for the EA).

\subsubsection{Conclusions}

In this section, the design methodology has been modified in order to include the possibility of following a dataflow paradigm.

The main contribution of this section in terms of development has been the adaptation of the discussed SA-based evolvable image filter to this dataflow paradigm. In addition, the whole system has been ported to a new development platform-Vivado-and to a new FPGA technology-Xilinx 7 Series. This has resulted in two products of the development work: a BRAMbased module similar to the one developed in $\$ 5.3$, which is interesting from the point of view of portability (although not of much academic interest), and a stream-oriented module which opens the door to new potential projects due to its interoperability with preexisting and third-party modules, thanks to the adoption of a widely used interconnection standard and communication paradigm.

Both modules have been implemented and tested in order to assess their validity and correct operation. The BRAM-based module with six $8 \times 8$ SAs in parallel has been successfully tested for frequencies of up to $250 \mathrm{MHz}$, which is the maximum that can be configured directly in Vivado for this FPGA without manually modifying the PLL parameters. However, timing analysis tools report maximum frequencies of about $300 \mathrm{MHz}$, and as it was seen with the Virtex5 implementation, these limits are likely to be too pessimistic, so further research on the maximum achievable frequency and overall evolution speed is suggested as a future research line.

The stream-oriented module has been tested for frequencies of up to $165 \mathrm{MHz}$, which is the maximum video pixel rate specified by HDMITM 1.2 [HDMI'05]. This is enough to cover the $148.5 \mathrm{MHz}$ pixel clock frequency necessary for a $1920 \times 1080 \mathrm{px}$ video stream at 60 frames per second as 
specified by [EIA/CEA-861-B, table 4]. ${ }^{27}$ Therefore, this module is suited for real-time high-definition video processing systems.

An example real-time video filtering application that integrates these modules into a complete video system will be presented in $\S 7.3$.

\footnotetext{
${ }^{27}$ This standard accounts for horizontal and vertical blanking periods, thus requiring a higher frequency than the theoretical minimum frequency of $1920 \times 1080 \times 60=124.416 \mathrm{MHz}$ that would be needed if pixels were transmitted in a compact manner without any blanking.
} 


\section{IMPROVEMENTS ON THE EVOLUTION TECHNIQUES}

With the adoption of the hardware design methodology described in $\$ 5.3$, the reconfiguration time and processing speed of the EHW system have been substantially improved, resulting in an evolution speedup from about 5 minutes to less than 1 . However, this may still be an excessively long time for certain applications.

This chapter studies several ways to reduce the evolution time. In section 6.1, different modifications on the EA are studied with the primary goal of making it converge faster, thus reducing the average number of generations necessary to reach a certain quality requirement. Additionally, some other considerations are taken into account that aim at reducing the reconfiguration time without affecting the evolution performance.

Then, section 6.2 focuses on exploiting the possibility of using multiple SAs in parallel that was introduced in $\$ 5.3 .4$, studying the correlation between the number of arrays and the evolution speed in order to determine the optimal number of arrays in this system.

\subsection{ACCELERATING THE CONVERGENCE OF THE EVOLUTIONARY ALGORITHM}

The evolutionary algorithm described in $\$ 3.1 .3$ is a very simple one that has been proven successful for the current EHW platform. However, it is far from optimal, requiring a considerably large number of generations to produce good results.

Until now, all optimization considerations have focused on improving the design techniques to achieve higher frequencies and thus faster evolution speeds, to a point that can hardly be further optimized as it is close to 
the switching frequency limit of the FPGA. However, exploring possible optimizations of the EA may result in a much greater reduction of the evolution time in a way that is completely independent of the hardware implementation, as there is much room for improvement on the current EA.

Thus, although this thesis focuses primarily on the hardware aspects of EHW, it has been considered that improvements on the EA represent an important aspect of EHW design.

Original contribution 6-1 A combination of evolutionary techniques that accelerate the evolution by minimizing the necessary number of evaluations to perform.

\subsubsection{Specification of the test case and initial results}

All the experiments and results in this section are based on the following system specifications:

- The hardware used for the experiments is the one specified in $\$ 5.3$, with an SA size of $\mathbf{8} \times \mathbf{8}$ PEs. This size has been chosen because it allows a whole LUT-based SA to fit within a single FPGA clock region (which simplifies the reconfiguration process) while offering a similar performance to other state-of-the-art solutions such as the $4 \times 8$ CGP used in [Vašíček'07a, Dobai'14].

- The training image set used for the evolution is the $128 \times 128$ pixel one used in $\$ 5.2$ (Figure 5-14), with $\mathbf{2 0 \%}$ salt and pepper noise, as used in previous work [Vašíček'07a, Salvador'13, Mora'15].

- The initial evolutionary algorithm is the $(1+\lambda)$-EA described in $\$ 3.1 .3$. This EA is run for 120000 generations; therefore a total of $120000 \times 8=\mathbf{9 6 0} 000$ candidate solutions are evaluated. ${ }^{1}$ As it was said, the mutation rate chosen for this EA is $K=3$, as this seems to yield satisfactory results.

\footnotetext{
${ }^{1}$ In previous work, the EA length was 800000 evaluations (100000 generations with 8 evaluations per generation). However, for the comparative analyses carried out in this section, the value has been increased to 960000 because of its divisibility by $4,6,8,10,12,15$, 30 ... which will simplify adjusting the offspring size and subdividing the EA (specifically, $\$ 6.2$ will justify the use of 12 SAs in parallel, requiring a number of evaluations that is a multiple of 12).
} 
A complete evolution run under these conditions takes 52 seconds to complete. The experimental results of the test case problem with the described EA are shown in Figure 6-1. In order to increase the statistical significance of the results, the EA has been run 1000 times with different initial random seeds. The plot shows how the overall fitness of all the EA runs improves compared to the number of candidate solutions evaluated.

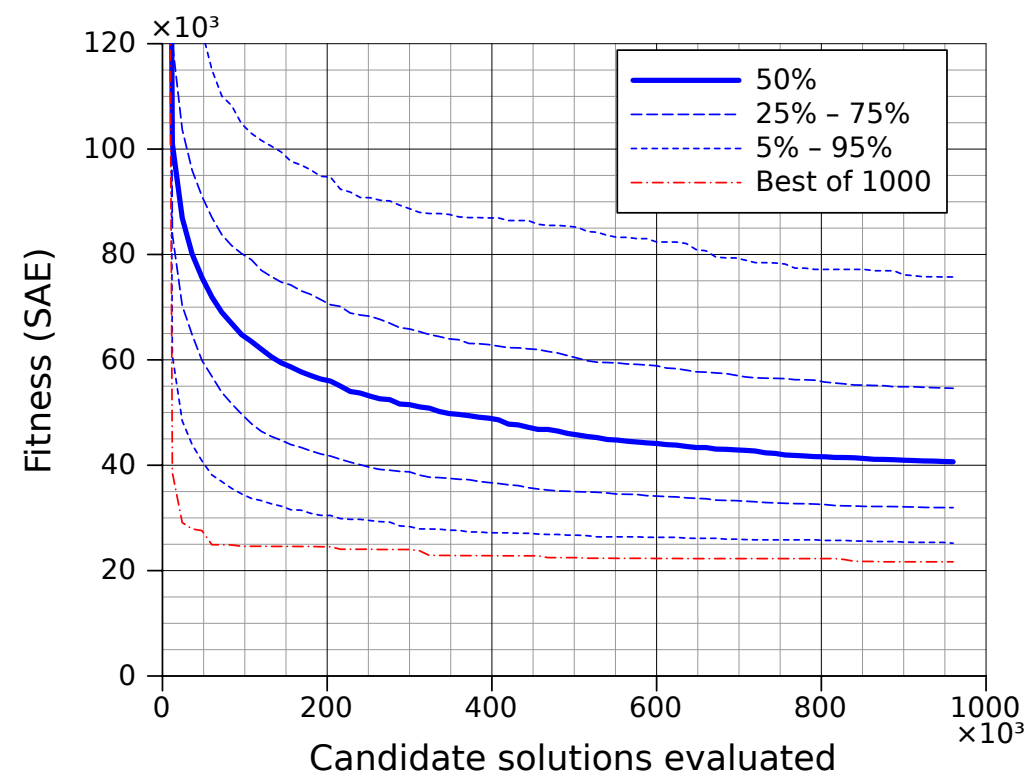

Figure 6-1: Plot showing the progress of the fitness value along the evolution, displaying the median value of the $1000 \mathrm{EA}$ runs (solid) as well as several confidence intervals (dashed). The best result among the 1000 evolutions is also shown.

As it can be seen, the median of the results at the end of the evolution is approximately 40000 . As a reference, the original image has an SAE with respect to the reference of 416297 , and the image filtered with a $3 \times 3$ pixel window median filter has an SAE of 84 187, which was outperformed more than $95 \%$ of the times.

However, the EA converges rather slowly by the end of the evolution, and the dispersion of the results is considerably high. This may be an indicator that the evolution is being easily stuck at local optima, making it very difficult for the EA to progress towards better solutions. 


\subsubsection{Finding the optimal mutation rate}

In $\S 3.1 .3$, a mutation rate of $K=3$ was chosen experimentally by performing a few tests on a $4 \times 4 \mathrm{SA}$, which showed that values between 2 and 5 yielded good results. However, as the array size increases, it becomes important to analyze the mutation rate to see if improvements can be obtained by varying it. Additionally, it should be noted that larger mutation rates involve performing a larger number of reconfigurations, increasing the reconfiguration time and thus slowing down the evolution. ${ }^{2}$ Therefore, it would be interesting to make the mutation rate as small as possible as long as it does not affect the obtained quality.

Figure 6-2 shows the results of the EA with different mutation rates, performing 1000 runs for each of them. Since the EA is still rather slow and a high number of tests are involved, the EA has been run for only 30000 generations (240 000 evaluations).

As can be seen, the best results are obtained for mutation rates of 2 and 3, with nearly identical results. A mutation rate of 1 gives very poor results, causing the evolution to rapidly stall. Mutation rates of 4 or more make the evolution converge more slowly, and thus yield worse results for the same evolution time. Additionally, as it has been stated, high mutation rates cause a longer reconfiguration time. Therefore, given that values of 2 and 3 produce the best results, a mutation rate of 2 becomes a more reasonable choice.

In order to ensure that the difference between these results is statistically meaningful and not a result of an excessively small sample size (even for a size of 1000 values), a two-tailed Student's $t$-test [Student'08] has been performed. This test yielded a p-value of 0.92 for $K=2$ versus $K=3$, not indicating statistical significance, and less than $10^{-16}$ for $K=2$ versus any other value, indicating that the apparent difference seen in Figure 6-2 is statistically significant.

It should be noted that this applies to SAs with $8 \times 8$ PEs; larger sizes would probably require greater mutation rates, so separate experiments should be made for those sizes. ${ }^{3}$

\footnotetext{
${ }^{2}$ Right now, the reconfiguration time only represents less than $20 \%$ of the total (as was seen in Table 5-9), making this improvement not too important; however, this fraction will acquire a larger weight when parallelization is introduced in $\$ 6.2$.

${ }^{3} \mathrm{An}$ analysis of the optimal mutation rate for different array sizes is carried out in [Barajas'17].
} 

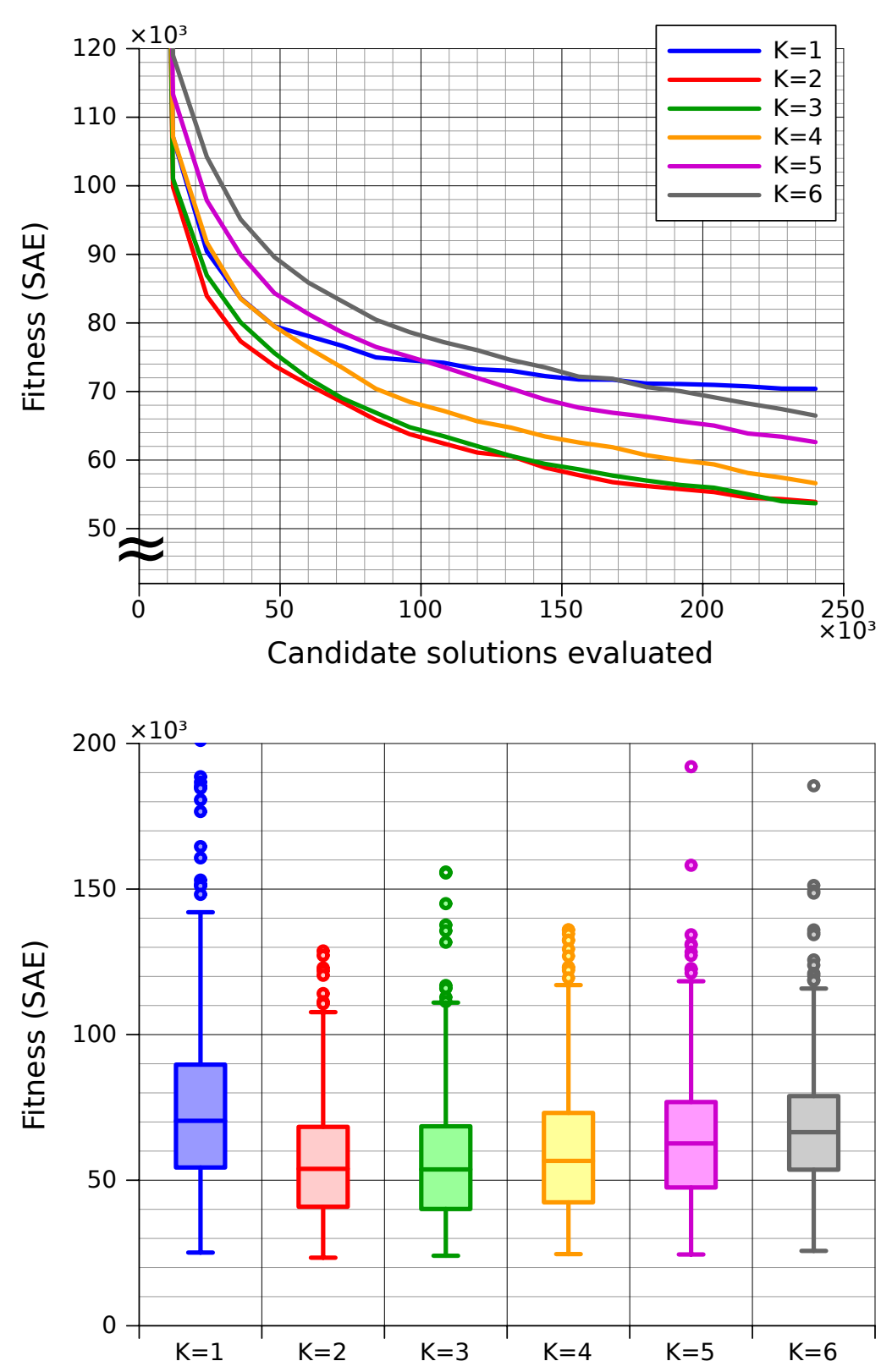

Figure 6-2: Results obtained for different mutation rates, showing the median value of the fitness (top) and the fitness distribution after evolving for a total number of 240000 evaluations (bottom). 


\subsubsection{Effect of the offspring size}

Another parameter that has been subject of study is the offspring size $(\lambda)$ of the $(1+\lambda)$-EA, this is, the number of children tested in each generation.

So far, the employed EA has used an offspring size of $\lambda=8$. Choosing large offspring sizes is typically done in evolution strategies, which are EAs based on real numbers where a small change typically causes an improvement or regression with similar probabilities. However this is not the case for the EA used here, which is closer to a genetic algorithm, where the probability of a change resulting on an improvement is relatively small.

Figure 6-3 compares the (1+8)-EA with a $(1+1)-E A$, for the same number of evaluations (not generations), both with $K=2$. As can be seen, both algorithms yield very similar results. This suggests that the offspring size does not affect the outcome of the EA for the same number of evaluations.

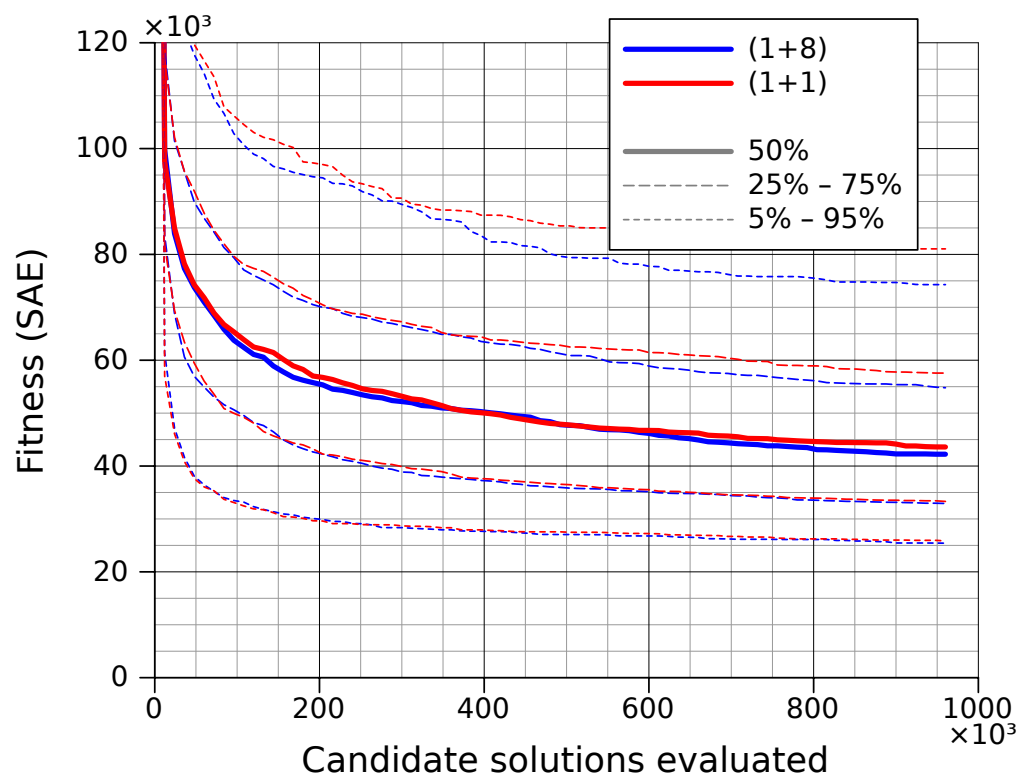

Figure 6-3: Results for a (1+8)-EA run for 120000 generations and a (1+1)-EA run for 960000 generations. Both algorithms perform the same number of evaluations (960 000), and thus take the same time to complete.

Given this result, the $(1+1)$-EA has been chosen over the $(1+8)$-EA in order to simplify its implementation and further modifications. Nevertheless, both strategies are equally valid, so an $(1+8)$-EA (or any other $(1+\lambda)$-EA) could be used if needed (for example to exploit parallelizability over multiple arrays, as is done in [Dobai'14]). 


\subsubsection{Increasing the diversity to avoid the effect of local optima}

The main problem of the original EA is that it is prone to getting stuck at a local optimum. This causes the EA to barely improve after a certain number of generations.

Figure 6-1 showed that the dispersion of the fitness results at the end of the evolution is rather high, with their median value being at 40000 but $90 \%$ of the results ranging from 25000 to 75000 . The curves near the end have a very reduced slope; therefore the evolution could be made shorter without this having a great impact on the result.

An easy way to obtain better results is to repeat the evolution multiple times, and pick the best result. For example, although the probability of getting a result below 32000 (25th percentile) is only $25 \%$, the probability of having any of 5 results below that value is $76 \%$. This involves running the EA multiple times, but since the gain towards the end of the evolution is small, it would be possible to shorten each of the evolutionary runs.

Figure 6-4 shows the median fitness of the best of $n(1+1)$-EA evolutions for a certain number of evaluations, with $n$ from 1 to 64 . The horizontal axis on the left plot represents the combined number of evaluations for the $n$ evolutions, and is therefore proportional to the time required to perform the whole training; the vertical orange line marks 960000 evaluations, detailed on the right plot. The fitness values shown on these plots have been estimated from the data obtained for a single (1+1)-EA using the formula

$$
Q_{n}(p)=Q_{1}(1-\sqrt[n]{1-p})
$$

with $p=0.5$, where $Q_{1}(p)$ is the quantile function of the fitness (i.e., the value for which there is a probability $p$ of obtaining a fitness lower than that value $)^{4}$ for a single (1+1)-EA, and $Q_{n}(p)$ is the quantile function for the "best of $n$ " approach.

As can be seen in Figure 6-4, performing 2 to 8 proportionally shorter evolutionary runs yields better results than performing a single one. Higher numbers of evolutionary runs show worse performance for a small number of evaluations since excessively short evolutions tend to yield bad results; however, this changes for longer evolution times. Specifically, for 960000 evaluations, the optimum is between 8 and 12 runs. $^{5}$

\footnotetext{
${ }^{4}$ For example, $Q(0.5)$ is the median, and $Q(0.25)$ and $Q(0.75)$ are the first and third quartiles.

${ }^{5}$ In view of this, any number of evolutionary runs between 8 and 12 would be a reasonable choice. Nevertheless, in $\$ 6.2$ it will be seen how using 12 SAs in parallel yields an optimal performance, motivating the choice of 12 evolutionary runs to simplify the dispatching.
} 

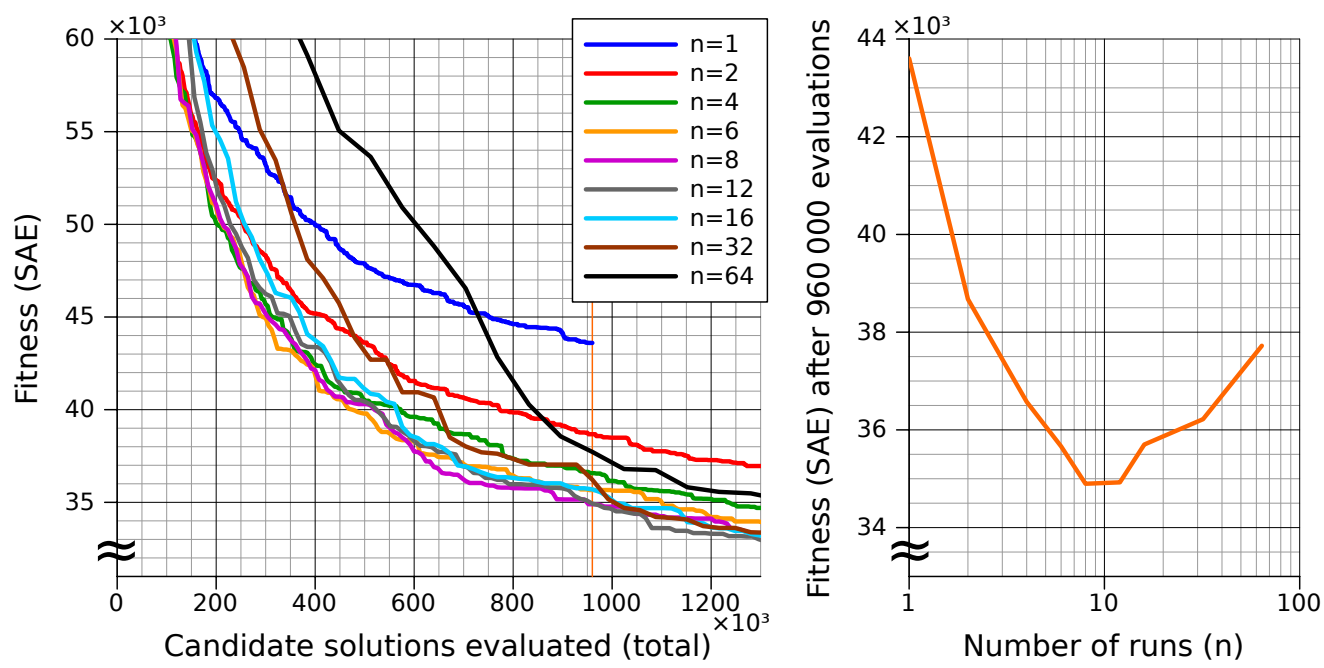

Figure 6-4: Median fitness of the best of $n$ evolutions over time (left) and after 960000 candidate solution evaluations (right).

Figure 6-5 shows the experimental results of selecting the best of $12(1+1)$ EA evolutionary runs of 80000 generations each. As can be seen, the new strategy outperforms the old one after 150000 evaluations, and can obtain results similar to those of the old one in half as many evaluations; additionally, the dispersion of these results is smaller.

In addition to obtaining better results, the new strategy is easily parallelizable, being able to distribute the evolutionary runs across multiple SAs which may even be on different devices; nevertheless, it can also be executed on a single device with a single SA by sequencing all the evolutionary runs, memorizing the final result of each one.

Original contribution 6-2 A method for finding the optimal number of parallel runs based on the quantile function for a single one, providing an improved speedup and, almost more importantly, a reduction in the dispersion of the results. 


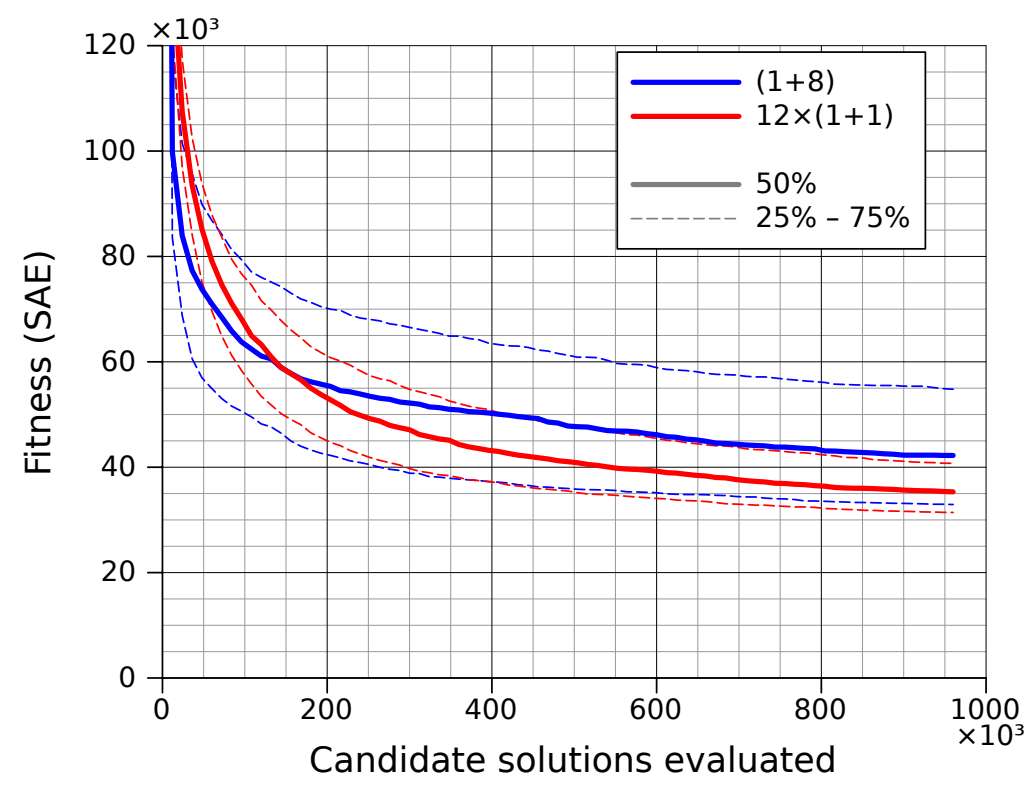

Figure 6-5: Comparison of the original (1+8)-EA with 120000 generations with the best of $12(1+1)$-EA with 80000 generations. Both strategies evaluate a total of 960000 candidate solutions.

\subsubsection{Promoting good evolutions}

Although the strategy described in $\$ 6.1 .4$ reduces the effect of the EA getting stuck at local optima, there is still a risk that some of the 12 evolutionary runs get stuck, becoming of no use for the rest of the evolution. Being able to remove these evolutionary runs and giving advantage to the rest would improve the result.

A way to achieve this is to execute all the evolutionary runs concurrently, performing an exchange every 400 generations. In each exchange, the evolution with the worst fitness is considered to be likely stuck and is terminated, and the one with the best fitness is considered as a promising evolution and is forked in order to improve the chances of success (Algorithm 6-1). This approach is similar to the island model described in [Sudholt'15], except that each "island" (evolutionary run) consists of a single individual and only one island migrates its individual to another one in each exchange.

As can be seen in Figure 6-6, this modification further improves the results. The new strategy is able to achieve the results of the original one in 192000 evaluations. Therefore, the evolution can be made 5 times shorter while still obtaining similar results. 


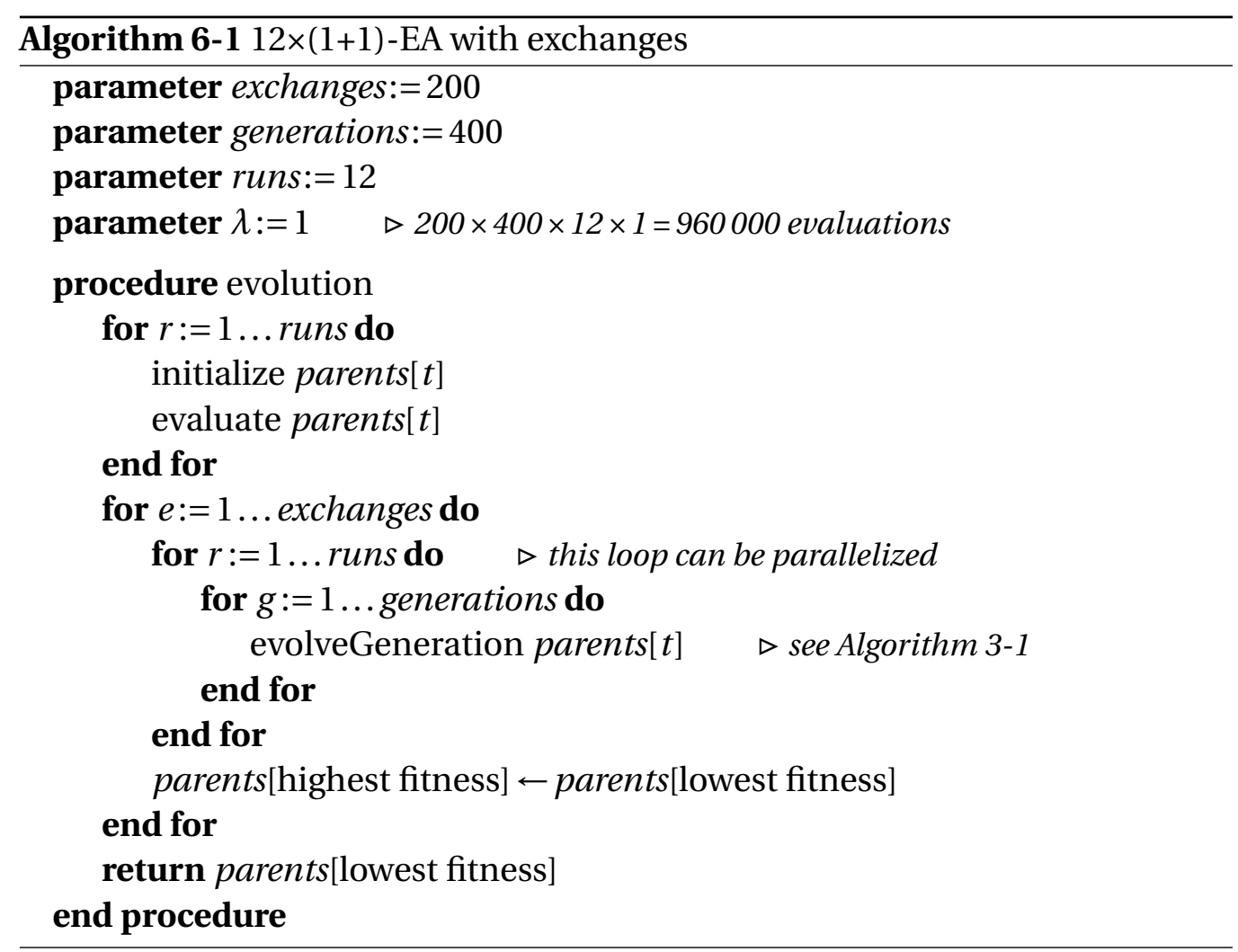

The aforementioned interval length of 400 generations between exchanges has been chosen because it achieves the best results for an evolution length of 192000 evaluations, as shown in Figure 6-7. ${ }^{6}$ Nevertheless, as it can be seen, this optimal point varies depending on the length of the evolution, becoming smaller for shorter evolutions. Therefore, the choice of the optimal exchange interval is subject to the planned evolution length.

\footnotetext{
${ }^{6}$ The exact value of this interval length does not have a great impact in the results. For example, in [Mora'18b], an interval length of 1000 generations rather than 400 was used since an exhaustive analysis of the optimal exchange interval had not yet been performed; nevertheless, the results are similar to the ones obtained here.
} 


\subsection{ACCELERATING THE CONVERGENCE OF THE EA}

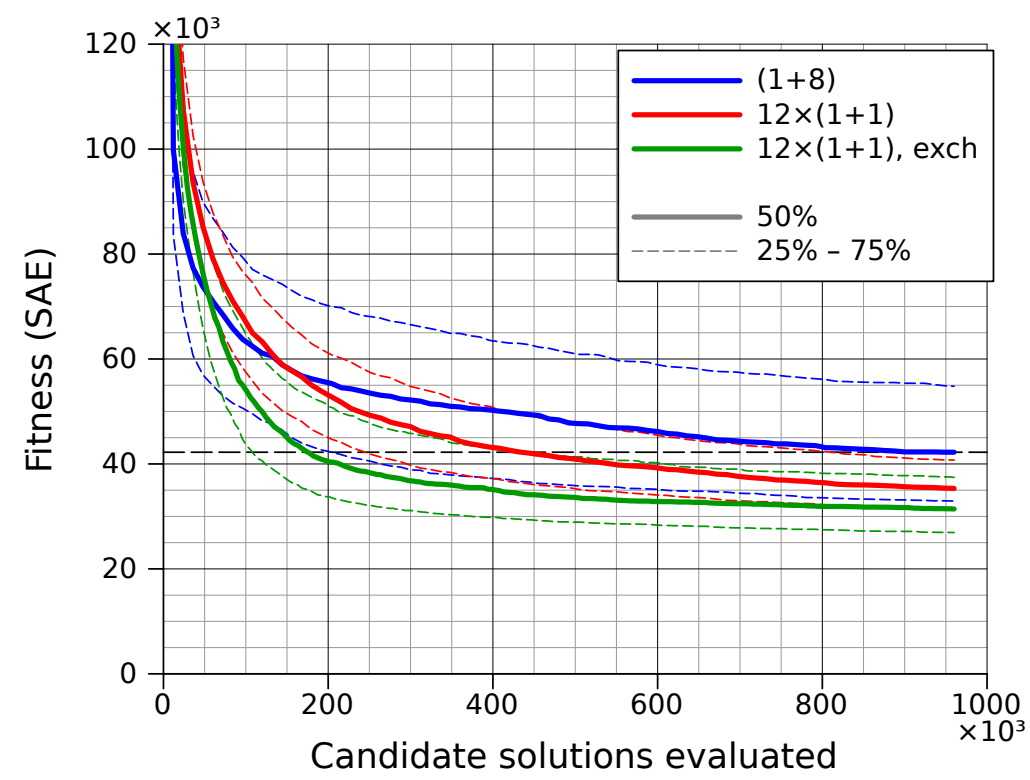

Figure 6-6: Comparison of the original (1+8)-EA, the best of $12(1+1)-\mathrm{EA}$, and $12(1+1)$ EA with an exchange every 400 generations (4800 evaluations), all with $K=2$. The horizontal dashed line marks the median fitness obtained with the original $(1+8)$-EA with $K=2$ (42346).

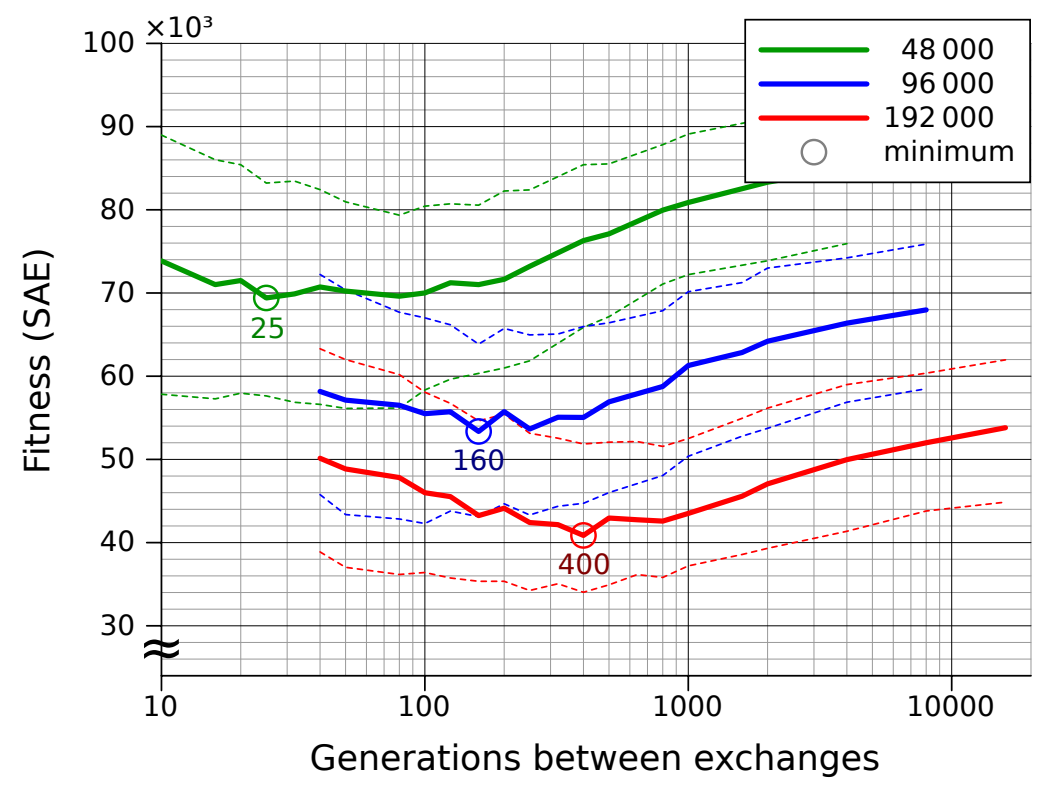

Figure 6-7: EA performance at different exchange intervals for evolution lengths of 48000,96000 , and 192000 evaluations 


\subsubsection{Restricting mutations to a single column}

Lastly, a way to reduce the time spent in reconfiguration is to force all the mutations to be on the same column of the SA. Since the reconfiguration engine described in $\$ 4.1$ writes the PE configuration in whole columns, forcing the mutations to be in the same column reduces the number of columns to reconfigure, which results in the reconfiguration time being reduced by approximately a $30 \%$. This strategy is similar to the one used in [Dobai'14], where mutations are inserted on individual frames of the FPGA configuration memory.

As can be seen in Figure 6-8, this modification does not cause any penalty on the fitness.

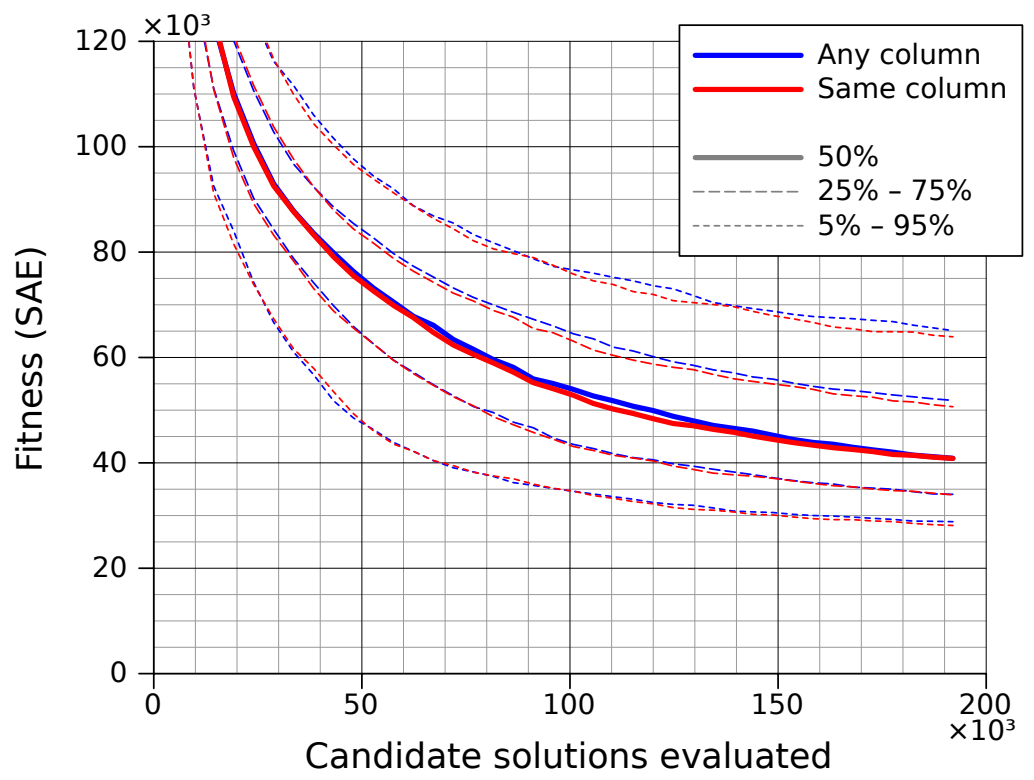

Figure 6-8: $12 \times(1+1)$-EA with exchanges, with $K=2$, comparing the results of mutating PEs and selectors in any column and restricting them to a single column.

\subsubsection{Evaluation of results and conclusions}

By optimizing the EA, the number of evaluations (and therefore the overall evolution time) can be reduced $\mathbf{5}$ times while still achieving similar results.

By reducing the mutation rate and restricting all mutations to a single column, the reconfiguration time has been reduced as well. Currently, the reconfiguration time is small compared to the time spent filtering the image, 
as it was seen in Table 5-9, so these savings are not too beneficial for now; nevertheless they will have a greater weight once the parallelization improvements described in $\$ 6.2$ are applied.

These two reductions in number of evaluations and reconfiguration time have reduced the total evolution time from 52 seconds to less than 10 seconds. The resulting implementation obtains results comparable to previous work [Dobai'14] using 6 times fewer evaluations. ${ }^{7}$

\subsection{EXPLOITING PARALLELISM}

In $\$ 5.3$, several efforts are made in order to maximize the operating frequency of the SA-based hardware module, thus incrementing its throughput and reducing the total evolution time, to a point where this frequency can barely be further improved.

Another way to increase the throughput of this module is to evaluate multiple solutions in parallel. This is done for example in [Dobai'14], where, by using six CGP arrays in parallel, the candidate solution evaluation speed is nearly duplicated with respect to a single array solution.

This section studies how this strategy can benefit EHW systems.

\subsubsection{Analysis of the parallelizability of the system}

As it was said in $\$ 5.3 .4$, the reduction of the PE size has allowed the possibility of implementing multiple SAs in parallel. Ideally, if the EHW system were fully parallelizable, the evaluation speed would be proportional to the parallelization factor. However, not all parts of the algorithm can be parallelized, making the growth in speed nonlinear.

Table 6-1 shows a breakdown of the evolution time per candidate solution evaluated. ${ }^{8}$

\footnotetext{
${ }^{7}$ In [Dobai'14], a Lena image corrupted with $5 \%$ salt and pepper noise is used, obtaining a median fitness value of 11863 after 300000 candidate solution evaluations (50 000 generations with 6 evaluations per generation). The architecture and EA presented here achieve that value for that test image in only 50000 evaluations.

${ }^{8}$ This table has updated values of mutation and reconfiguration times with respect to Table 5-9, resulting from the mutation and reconfiguration optimizations introduced in $\S 6.1$.
} 


\begin{tabular}{lrr} 
Stage & \multicolumn{2}{c}{ Time $(\mu \mathrm{s})$} \\
\hline Mutation & 1.5 & $(3.1 \%)$ \\
Reconfiguration & 5.2 & $(10.6 \%)$ \\
Filtering & 42.0 & $(85.7 \%)$ \\
Selection & 0.3 & $(0.6 \%)$ \\
\hline Total & $\mathbf{4 9 . 0}$ &
\end{tabular}

Table 6-1: Evolution time breakdown in microseconds for a single evaluation

As it can be seen, most of the evolution time is spent in filtering the image; implementing $n$ SAs in parallel would effectively reduce the time used in this stage by a factor of $n$. The rest of the parts (mutation, reconfiguration, and selection) are required to be run sequentially-since there is a single processor and a single reconfiguration port-, and thus cannot be parallelized.

As it can be derived from Amdahl's law [Amdahl'67, Gustafson'88], the overall speed of a parallelized system can be calculated as

$$
v_{n}=\frac{n}{t_{n}}=\frac{n}{1 \cdot t_{\mathrm{P}, 1}+n \cdot t_{\mathrm{S}, 1}}=\frac{1}{\frac{t_{\mathrm{P}, 1}}{n}+t_{\mathrm{S}, 1}}
$$

where $n$ is the parallelization factor, $t_{n}$ is the total time required to perform $n$ evaluations, $t_{\mathrm{P}, 1}$ is the time used by parallelizable operations, and $t_{\mathrm{S}, 1}$ is the time used in a single evaluation by operations that cannot be parallelized. This implies the existence of a limit in the speed that cannot be reached no matter how much the system is parallelized, given by

$$
v_{\max }=\lim _{n \rightarrow \infty} v_{n}=\frac{1}{\frac{t_{\mathrm{P}, 1}}{\infty}+t_{\mathrm{S}, 1}}=\frac{1}{t_{\mathrm{S}, 1}}
$$

this is, the maximum speed is limited by the time spent by sequential operations.

In our EHW system, $n$ corresponds to the number of SAs; $t_{\mathrm{P}, 1}$ is the filtering time $(42 \mu \mathrm{s})$, and $t_{\mathrm{S}, 1}$ is the sum of mutation, reconfiguration, and selection times $(7 \mu \mathrm{s})$. Figure $6-9$ shows the result of applying the previous formulas to this system.

As it can be seen, the parallelized system approaches an asymptote at 143000 evaluations per second as more SAs are added, 7 times faster than with a single SA. However, this approach is quite slow: with 6 SAs, only $50 \%$ of this speed is reached; 18 SAs would achieve $75 \%$ of it.

The solution proposed in [Mora'15] uses 8 SAs, as this seemed to be a good compromise value between resource usage and performance. Said solution 


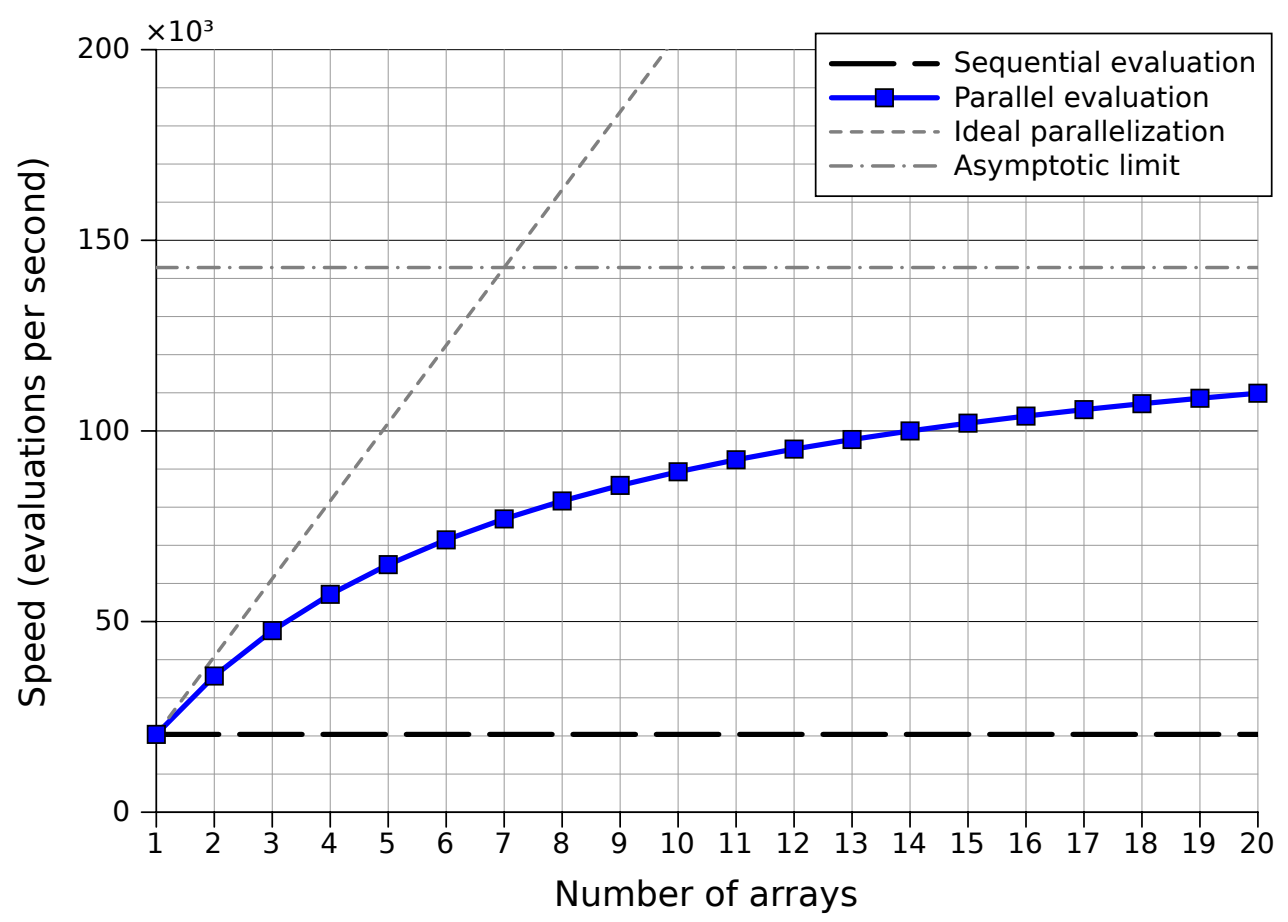

Figure 6-9: Number of evaluations per second achieved with multiple SAs filtering in parallel, compared with using a single SA at a time and an ideal "totally parallelized" model in which all stages are parallelized (this could be achieved using multiple FPGAs).

achieves a speed of 81000 evaluations per second. Using more arrays would have involved a great increase in the resource usage for a small performance gain: having twice as many arrays would only improve the speed by $27 \%$.

\subsubsection{Improved scheduling}

One aspect that has not been considered in $\S 6.2 .1$ is the fact that the SAs are idle during reconfiguration. This is obviously necessary for a system with a single SA, since the array cannot operate until it has been fully reconfigured. However, on a multi-SA system, once an array has been reconfigured, it could start working immediately without waiting for the rest of them.

Launching several SAs asynchronously is not possible in the current implementation, since it uses a common memory for input and reference images. Nevertheless, it is possible to select only a few of the SAs and their corresponding fitness computing units to perform the evaluation, ignoring the output of the rest. 
Therefore, the following strategy has been envisioned: the total number of SAs are divided into two groups, and while half of them are being reconfigured, the other half are already processing an image, as demonstrated in Figure 6-10.

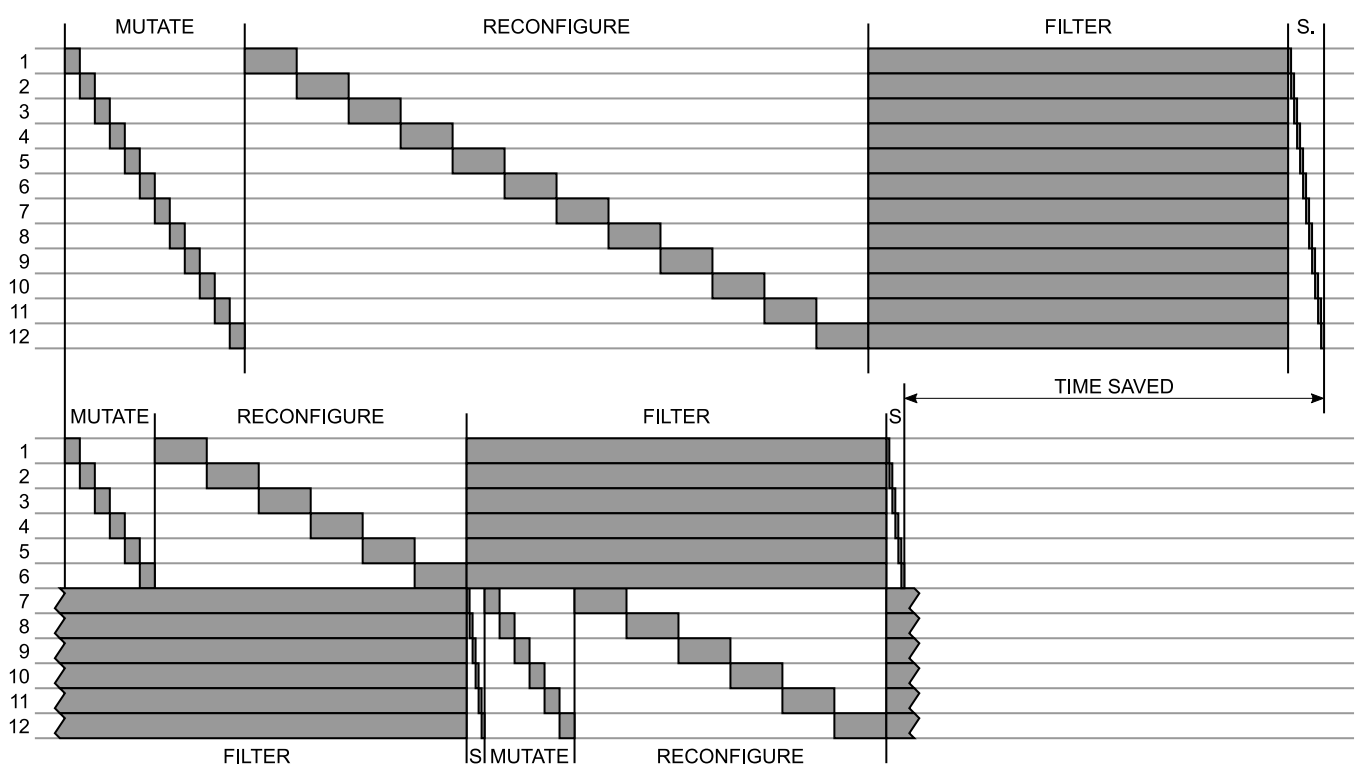

Figure 6-10: Comparison of both scheduling strategies for a 12 SA system. Top: former scheduling, showing a complete mutate-reconfigure-filter-select cycle. Bottom: new "half and half" scheduling, showing a complete cycle for arrays 1 to 6 and two fragments of consecutive cycles for arrays 7 to 12 .

In order to take full advantage of this interleaving of operations, the evolutionary processes on both halves must be independent, so that the configuration of each half is never dependent on the result of the other half. For example, a 6+6 SA implementation (as depicted in Figure 6-10) could use this strategy on a $12 \times(1+1)$-EA or a $2 \times(1+6)$-EA, since this would allow running half of the concurrent evolutionary runs on one SA group and the other half on the other group; but a single $(1+12)$-EA would barely benefit from this improvement, as all the operations in one generation need to finish before deciding the configurations to be tested on the next generation.

This strategy is not always beneficial to the evaluation time, as it makes the filtering stage twice as slow since only half of the SAs are working simultaneously. However, it allows the filtering stage to overlap with the mutation and reconfiguration ones, saving the time corresponding to the shortest of the two. The overall evolution speed obtained with this strategy can 
be determined by the formula

$$
v_{m+n}=\frac{m+n}{t_{m+n}}=\frac{m+n}{\max \left(t_{\mathrm{P}, 1}, m \cdot t_{\mathrm{S}, 1}\right)+\max \left(t_{\mathrm{P}, 1}, n \cdot t_{\mathrm{S}, 1}\right)}
$$

where $m$ and $n$ are the sizes of the two groups (which may be different; for example, if the total number of arrays is odd, one group could have one more element than the other).

Figure 6-11 shows the behavior of this formula in the context of the current system. As it was expected, the performance of this solution for a low number of SAs is worse than the previous one; however, it grows linearly with the number of SAs, surpassing the previous approach for more than 6 SAs and reaching the theoretical limit at 12 SAs.

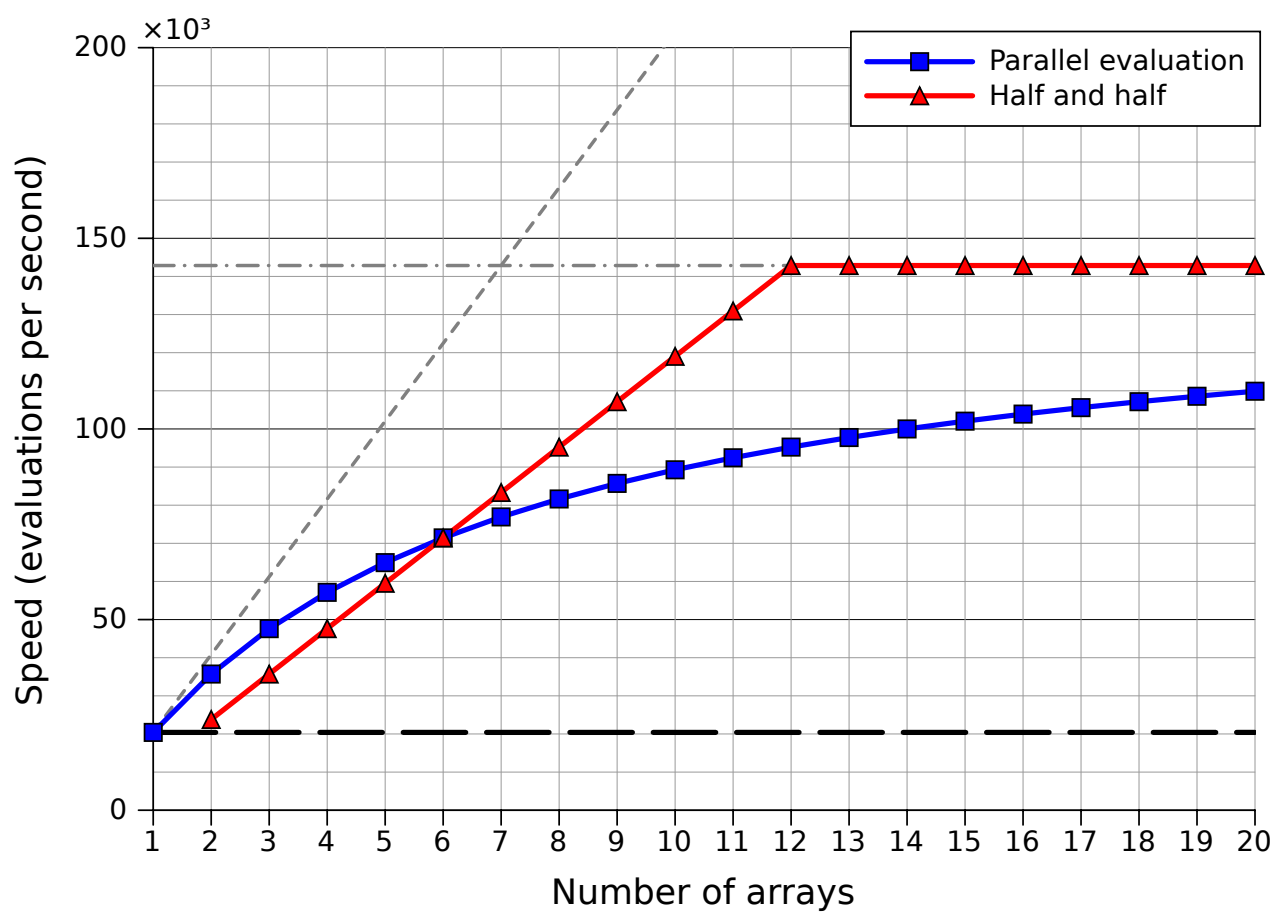

Figure 6-11: Comparison of the previous parallelization approach with the half-andhalf scheduling

The maximum speed is reached when the time of sequential operations surpasses the time of parallelizable operations, since the former becomes the limiting factor. Thus, the number of arrays at which this solution reaches the maximum theoretical speed (so that adding more than these many arrays to the 
system will not improve its performance at all) can be calculated as

$$
n_{\max }=2 \cdot \frac{n_{\max }}{2}=2 \cdot \min \left\{m \in \mathbb{N} \mid m \cdot t_{\mathrm{S}, 1} \geq t_{\mathrm{P}, 1}\right\}=2 \cdot\left\lceil\frac{t_{\mathrm{P}, 1}}{t_{\mathrm{S}, 1}}\right\rceil
$$

where $\lceil x\rceil$ is $x$ rounded up. The critical number of arrays at which half-and-half scheduling becomes preferable over the sequential one is given by

$n_{\text {crit }}=\min \left\{n \in \mathbb{N} \mid t_{\frac{n}{2}+\frac{n}{2}} \leq t_{n}\right\}=\min \left\{n \in \mathbb{N} \mid 2 \cdot t_{\mathrm{P}, 1} \leq t_{\mathrm{P}, 1}+n \cdot t_{\mathrm{S}, 1}\right\}=\left\lceil\frac{t_{\mathrm{P}, 1}}{t_{\mathrm{S}, 1}}\right\rceil$

i.e., the half-and-half strategy will be preferable if the number of arrays is $n_{\text {crit }}$ or more.

In the case at hand, $t_{\mathrm{P}, 1}=42 \mu \mathrm{s}$ and $t_{\mathrm{S}, 1}=7 \mu \mathrm{s}$; thus, these two formulas yield the aforementioned figures of $n_{\max }=12$ and $n_{\text {crit }}=6$. Therefore, the current EHW system achieves its maximum performance with 12 SAs in parallel, and the optimal reconfiguration strategy is the sequential one if it has less than 6 SAs and the half-and-half one if it has 6 or more.

This optimal number of 12 SAs justifies the choice of using specifically 12 concurrent evolutionary threads that was made in $\$ 6.1 .4$, since it simplifies the dispatching of threads across SAs. Nevertheless, any other $n \times(1+\lambda)$-EA strategy where $n$ is even and $n \cdot \lambda$ is a multiple of 12 would have been valid as well.

It should be noted that this strategy has a small penalty on the software time, increasing it from 1.8 to $2.0 \mu$ s per evaluation, and thus the effective speed that is reached with this approach is 139000 evaluations per second, slightly smaller than the predicted 143000 . This represents a speed gain of $45 \%$ with respect to the 95000 that would be obtained with the same 12 SA system if this improved scheduling were not used.

Original contribution 6-3 The use of only half of the available reconfigurable modules in a modular parallel DPR system while the other half processes data, and the criteria to determine the point at which this is a beneficial idea. 


\subsubsection{Evaluation of results and conclusions}

Although the parallelization strategy described in this section does not provide a proportional increase in the evaluation speed, a great speedup can be obtained by implementing up to 12 arrays in parallel and using half of them while the other half is reconfigured. This parallelization has accelerated the evolution of the current system nearly 7 times with respect to a single SA implementation, reducing the evolution time from the 10 seconds reported at the end of $\$ 6.1$ for a 192000 -cadidate evolution to 1.38 seconds without affecting the quality of the results.

The combination of optimized hardware architecture, improved EA, and optimized parallelization makes this system achieve results comparable to those obtained in [Dobai' 14 ] in only $0.36 \mathrm{~s}$, making this approach near 28 times faster.

For 12 or more SAs, mutation and reconfiguration times become the limiting factors, so the current approach cannot be further optimized unless these times are reduced. Both mutation and reconfiguration are limited by the microprocessor, so using a hardware implementation of the EA that communicates directly with the reconfiguration engine (as partially implemented in [Lanza'13]) would reduce this time. If this time were reduced to the theoretical minimum required by the reconfiguration engine $(2.8 \mu \mathrm{s})$, a theoretical speed of up to 350000 evaluations per second could be achieved by using 30 SAs in parallel; however these many SAs would occupy approximately $70 \%$ of the resources of the FPGA currently in use (Xilinx Virtex-5 LX110T), so it is possible that such an implementation requires a larger FPGA.

Nevertheless, this approach limits the range of evolutionary algorithms that can be used, as it is required that these algorithms be easily parallelizable, so for example a single (1+1)-EA (as suggested in $\$ 6.1 .3$ ) would not be able to take advantage of this improvement. Additionally, for an optimal utilization of this parallelization, the algorithm should be evenly divisible by the number of arrays, although there is still room for adjustments on the EA parameters.

Original contribution 6-4 A parallelized EHW system, together with a series of evolution and scheduling techniques, that can evaluate up to 139000 candidate solutions per second, the highest value reported so far for FPGA implementations of EHW to the best of the author's knowledge. 



\section{Chapter}

\section{EXPLORATION OF APPLICATIONS BASED ON THE CURRENT SYSTEM}

Although it has been proven that the system studied in this thesis can adapt to multiple different problems, most of the experimental results so far have focused on a single task: the removal of salt and pepper noise from an image. Nevertheless, this is a generic architecture that could be used in a multitude of applications and can potentially solve arbitrary problems; however, none of these real-life applications have been experimentally assessed yet.

This chapter examines the feasibility of using the studied system in different applications or situations in order to assess its versatility. In section 7.1, the system is tested in a more challenging scenario: training a noise filter in conditions in which the noise model is not known. This is a more realistic task, since normally the conditions at which an adaptive filter will need to work are not known beforehand, and therefore a training set (input and reference) cannot be provided; the system will need to obtain that information directly from its input.

Section 7.2 studies the performance of the system in a variety of tasks, some of them not related to noise removal but to different image enhancing and manipulating operations, with the purpose of assessing its generalizability. Additionally, several modifications to the system configuration such as larger input windows and different sets of PE functions are explored, in order to evaluate how these variations affect each different problem.

Finally, section 7.3 describes an example application that integrates the studied system into a larger design oriented to real-time high-definition video processing, demonstrating the usefulness of the system in real life designs. 


\subsection{NOISE-AGNOSTIC EVOLUTION FOR AUTONOMOUS VIDEO FILTERING SYSTEMS}

One question frequently heard in conferences, workshops, and seminars related to self-adaptive evolvable image filters is: this system is able to train given a noisy input and a noise-free reference, but what if we do not have those?

This question is far from trivial to address. One of the strong points of EHW is its application to create autonomous, self-adaptive systems. However, this is not the case if the training set needs to be supplied externally, as it would fail to be autonomous, or if the training set is fixed, which would make it not adaptive. Therefore, part of the research efforts in this thesis have been in the direction of finding a way to obtain a training data set in an autonomous manner. ${ }^{1}$

Since an adaptive system might be required to change under varying working conditions, the training data set should be obtained from the system input so that this training is performed according to such conditions in a specific moment. One possible way to achieve this on a video filtering system would be to have a physical reference the system can be pointed to, for example, a test card printed on a wall or on the ground. The system could then use the input received when recording this test pattern as a training input; the training reference would be the original test pattern. However, this approach may be infeasible in many cases, ${ }^{2}$ and it would require stopping the system from its normal operation to point its camera to a different location where the test pattern is.

Another possibility would be to perform a software-based filtering (which may be much slower than what would be achievable in hardware) on one of the images obtained from the video input, and then using the result as training reference for the EHW system, so that the system would work as a hardware accelerator of a task that is costly in software; however, this would increase the complexity of software and require some previous knowledge of how to filter the expected types of noise. Alternatively, the image could be analyzed (either in software or using specialized hardware) in order to identify

\footnotetext{
${ }^{1}$ The development of this noise-agnostic evolvable system was part of the M.Sc. thesis of the author [Mora'13a].

${ }^{2}$ An example would be satellite applications, which typically need to be autonomous and where having a physical test pattern may be hard to achieve. Nevertheless, patterns created on the Earth surface for satellite calibration do exist, so this option is not completely impossible.
} 
the noise type and intensity, but this would constrain the possible use cases to a limited set of noise types.

Due to the limitations of the aforementioned approach, the research has focused on finding a solution that is noise-agnostic, this is, that does not attempt to analyze or classify the noise, but rather uses it directly to generate a solution.

The use case that will be studied in this section is noise removal from video frames. Because of this, rather than relying on a single image for the experimental analysis, this section will use the Foreman video sequence (Figure 7-1), a 300-frame (10 seconds) test sequence often used in video filtering applications, and will apply each of the solutions obtained after each EA run to all of the frames in the sequence in order to obtain its overall fitness.

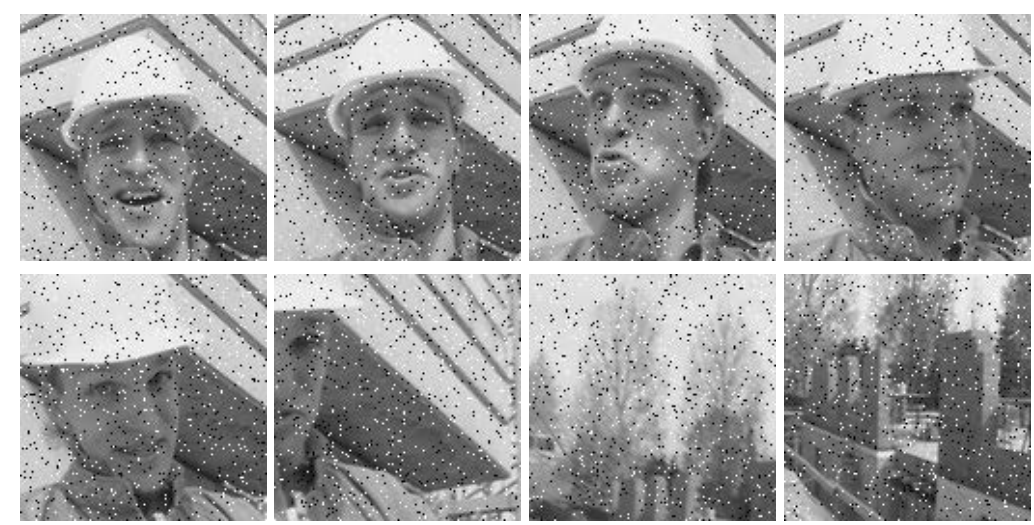

Figure 7-1: Some frames from the Foreman sequence, which has been cropped to $128 \times 128$ pixels and corrupted with $5 \%$ S\&P noise for the experiments.

\subsubsection{Usage of a noisy image as a reference}

The solution chosen in [Mora'13a, Mora'13b] for evolving a noise filter for a video input for which we do not have a golden reference consists in using a noisy image as the reference as well as the input. ${ }^{3}$ This approach is similar to the one used in [Zhou'98] to train an adaptive order statistic filter.

\footnotetext{
${ }^{3}$ This does not mean that input and reference are identical, only that they are derived from the same image and have the same type of noise.
} 
Original contribution 7-1 The use of two noisy versions of the same original image for training an EHW filter is an original contribution; nevertheless, there exists previous work using this technique for tuning other types of adaptive system [Zhou'98].

\section{Theoretical explanation}

This solution relies on the noise being random (and therefore cannot be used in cases where the noise is not random, such as dead pixels on a camera sensor).

If we make the system evolve using a noisy image as its training input and the same image with the same type of noise as training reference, it will try to remove the noise on the input and add the noise on the reference. However, since noise is random, the filter will not be able to infer the noise pattern that has to be added to the image in order to obtain the reference, and thus will only be able to remove the noise on the input. Therefore, although the fitness value obtained during the evolutionary process will be much worse than that obtained with a noise-free reference, the filter performance will actually be very good, comparable to that obtained with a noise-free reference, as will be demonstrated later.

Figure 7-2 illustrates this from the point of view of individual pixels for the case of salt and pepper noise. As can be seen, the system is able to evolve because corrupt pixels in the training input are usually uncorrupted in the training reference, so the system is able to figure out what these pixels should look like.

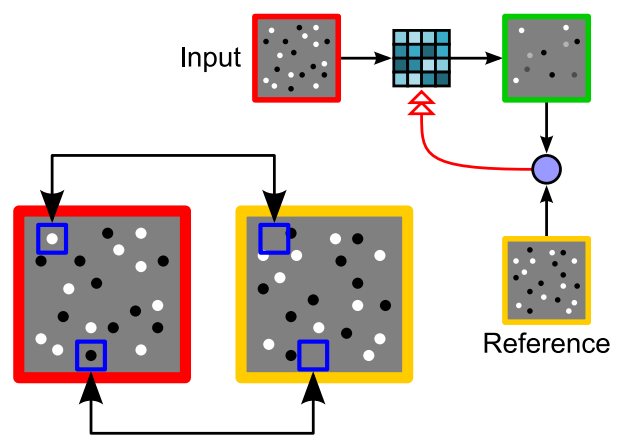

Figure 7-2: Evolution performed with a noisy image as a reference 


\section{Experimental results}

The strategy described above has been experimentally assessed under the system described in $\$ 3.1(4 \times 4$ PE SA), using a $(1+8)$-EA with 5 parallel runs (as described in $\$ 6.1 .4$ ) with a run length of 20000 generations per run (800 000 evaluations in total). The EA has been run a total of 300 times, using a different frame from the Foreman sequence as input and reference each time, both of them with $5 \%$ salt and pepper noise. At the end of each evolutionary run, the obtained filter is evaluated with each of the 300 frames of the sequence, calculating the SAE between the result and the original (noise-free) frame. The resulting $300 \times 300=90000$ SAE values are displayed in a box plot $^{4}$ in Figure 7-3 along with the SAEs at the input, the ones obtained with a median filter, and the ones obtained when evolving with a noise-free reference.

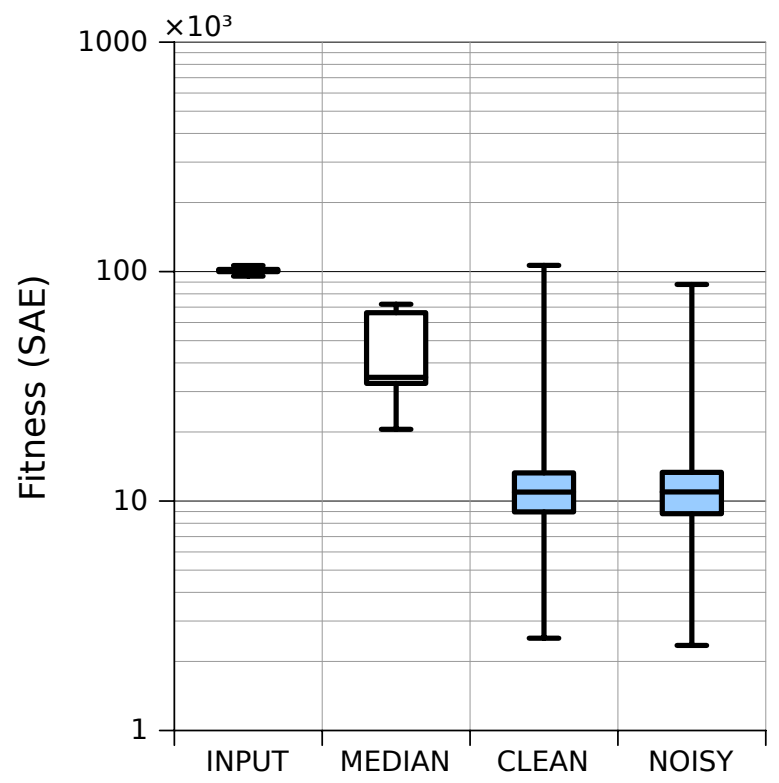

Figure 7-3: Results obtained by evolving using a noisy image as training reference, compared to the ones obtained with a median filter and an evolvable filter trained with a noise-free reference. The SAE between the noisy input images and the original noisefree images is also shown.

\footnotetext{
${ }^{4} \mathrm{~A}$ side effect of having these many values in a box plot is that the dispersion of its extremes becomes very high, making the whiskers give an unreliable visual idea of the overall dispersion, specially when comparing samples with different sizes. Therefore, one should not pay much attention to the length of the whiskers (minimum and maximum) and focus on the boxes instead (25-75\% quartiles).
} 
As it can be seen, the results are almost identical to those obtained using a noise-free reference. Furthermore, excluding extreme values, both approaches yield much better results than the median filter.

\section{Conclusions and remarks}

The results obtained are very promising, and show that it is possible to train the system even without having a noise-free training reference, using exclusively noisy images. Furthermore, this approach leads to solutions of similar quality as those trained with a noise-free reference.

However, this is not a realistic scenario for the case of real time video filtering, as it requires having two identical images with noise. This could be the case, for example, if the aim of the filter were to remove the noise of images due to transmission on a noisy channel, since the same image could be transmitted multiple times. Real time video captured with a noisy sensor, on the other hand, consists of a sequence of slightly different frames, and does not have the opportunity of repeating one of the frames.

\subsubsection{Usage of two consecutive noisy frames as training data set}

In most cases, a video filtering application will be used because it is necessary to filter a video stream targeting moving objects. Therefore, this video stream will consist of a sequence of similar but not identical images, with some parts of the image moving or changing in contrast from one frame to the other.

However, it is expected that two consecutive frames are similar enough to use them as training set despite of their small differences. ${ }^{5}$ Previous work, such as [Saeidi'05], relies on this similitude to implement image filters that remove noise by averaging consecutive frames. In contrast, the proposed approach uses two similar frames (with different noise patterns) for the evolution, with the resulting filter being applied to individual frames.

\footnotetext{
${ }^{5}$ This was informally assessed using the real time demo application described in $\$ 3.4 .5$, using two frames captured with its camera. The results were visually good enough, but may be biased due to the fact that the video frames were intentionally being captured at an appropriate time, when the filmed objects moved the least, rather than capturing two random frames.
} 


\section{Experimental results using two arbitrary consecutive frames}

The procedure for testing this approach is similar to the previous one: for each of the 300 frames of the video, a filter has been obtained by using that frame as input and the next frame as reference.

As it can be seen in Figure 7-4, contrary to what was expected, the results are very bad, worse than the median filter in most cases and even worse than the input for more than $25 \%$ of the cases. This is probably due to the video sequence presenting an excessive amount of motion, making each two frames too different to use as training set, leading to filters that try to distort the image to adapt to the motion or simply to evolutions that do not converge properly. This is solved in [Saeidi'05] by implementing motion compensation algorithms; however, implementing those algorithms in the current system might introduce an excessive complexity.

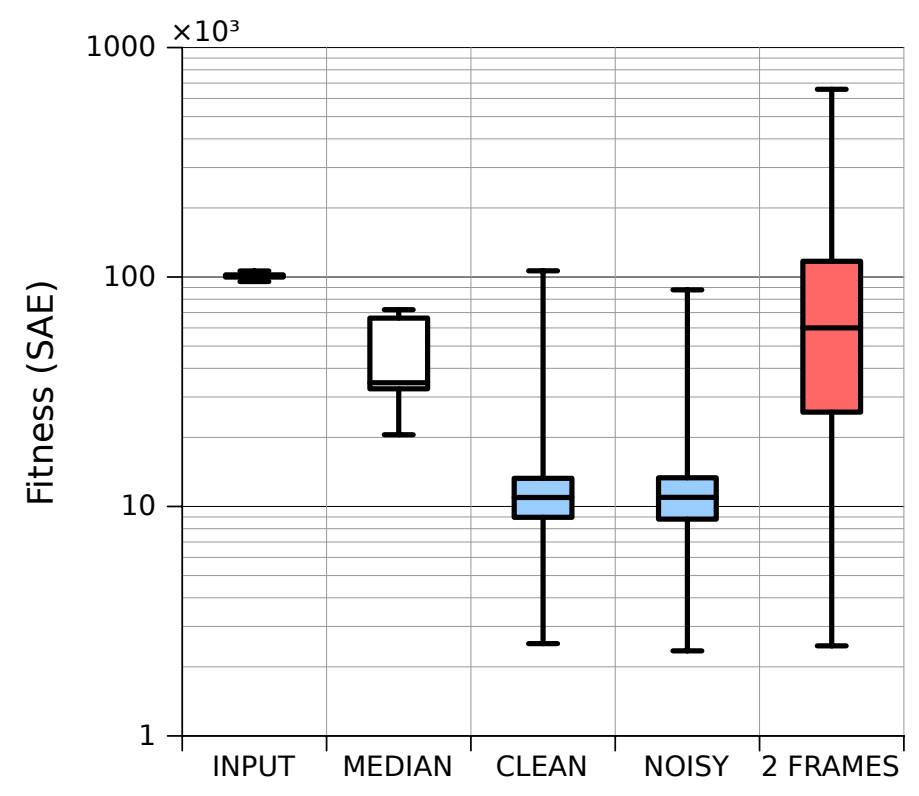

Figure 7-4: Evolution results using two consecutive frames as training input and reference respectively, compared to the previously obtained ones. 


\section{Experimental results selecting frames based on their similitude}

The solution chosen in order to overcome the bad results shown in Figure 7-4 is to perform a search of two frames that are as similar as possible and perform the evolution with said frames. Theoretically, this search should be performed over the original noise-free frames. However, as it can be seen in Figure 7-5, SAE between two noisy frames is strongly correlated with SAE between their noisefree counterparts. Therefore, the search for similar frames can be performed on the noisy video sequence, not requiring any additional input. The search can be performed using the fitness computing unit of the SA system by setting the SA to a copy filter, thus not requiring any additional hardware nor a long software processing time.

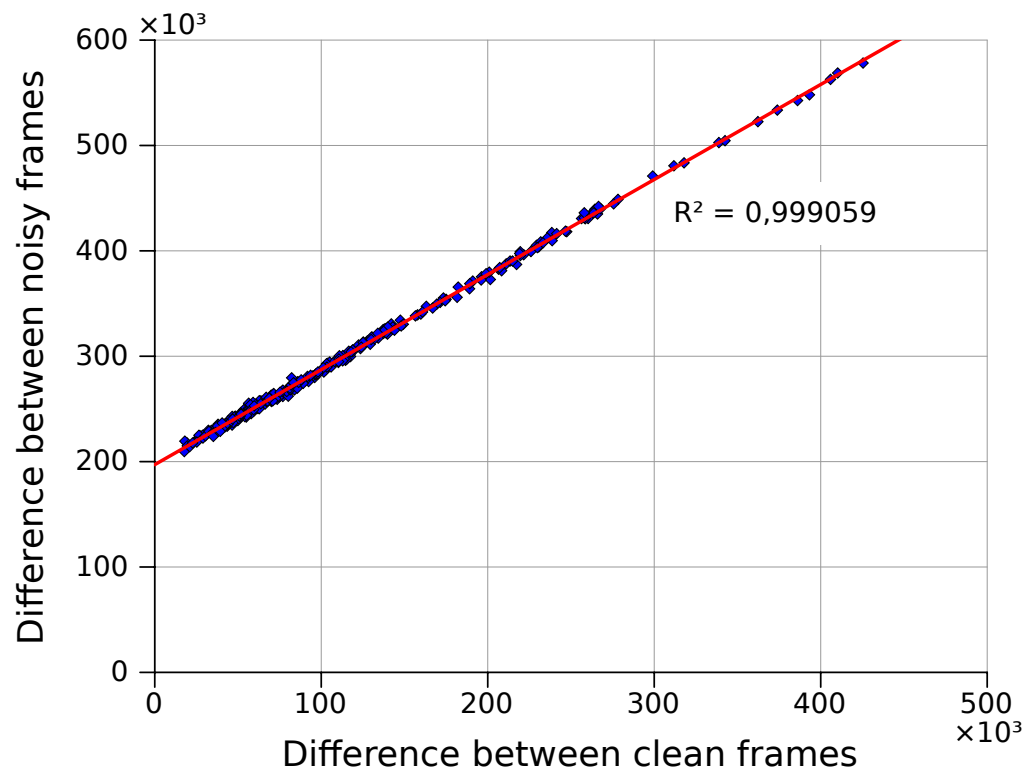

Figure 7-5: Correlation between SAE of noisy and noise-free frames. Each point represents one pair of frames in the sequence. As it can be seen, the deviation from a linear correlation is negligible.

The quality of the results will depend on the length of the sequence in which these two frames are searched: the longer the sequence, the higher the chances to find two similar frames, but the longer the time the system will be performing 
suboptimally. ${ }^{6}$ Figure 7-6 shows the results obtained with lengths of the search sequence of 10 frames $^{7}$ ( $0.3 \mathrm{~s}$ at 30 frames per second), 30 frames ( $\left.1 \mathrm{~s}\right), 60$ frames $(2 \mathrm{~s}), 100$ frames $(3.3 \mathrm{~s})$, and the whole 300 -frame sequence (10 s).

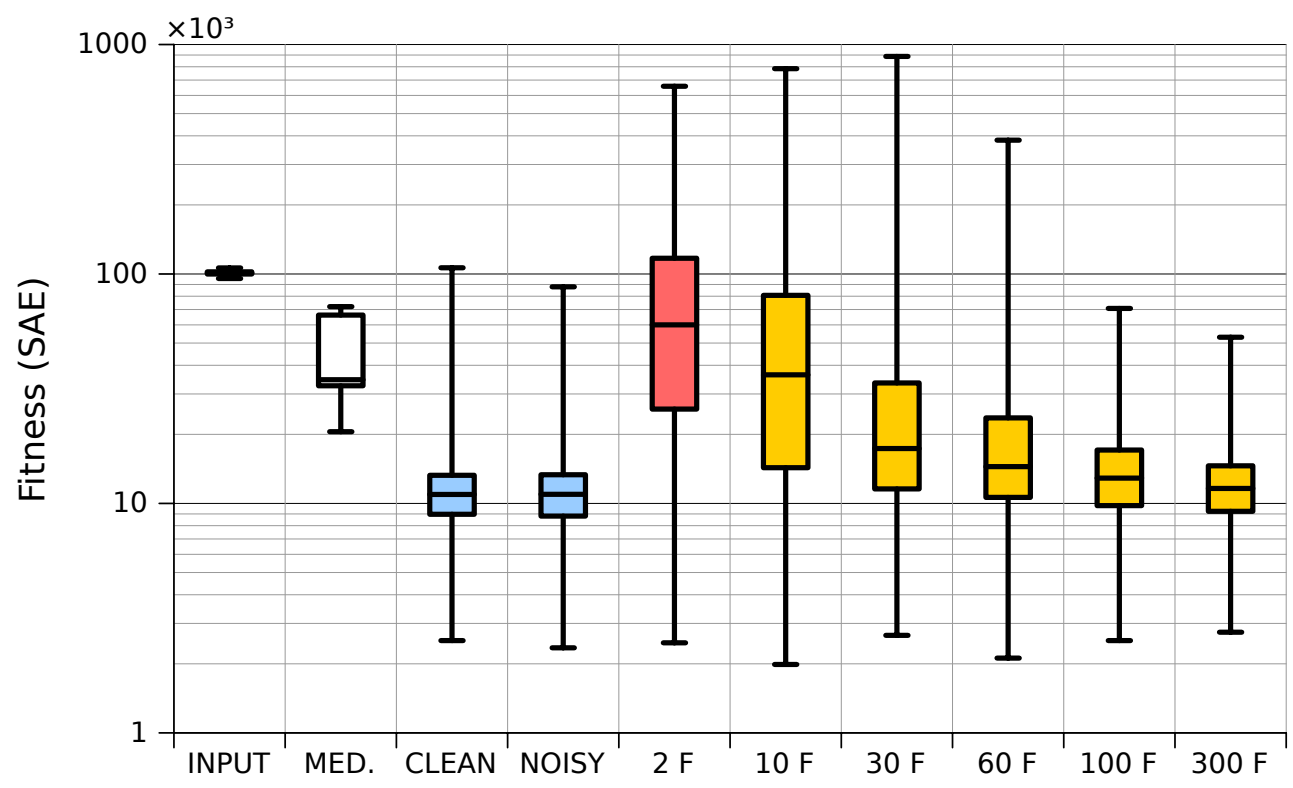

Figure 7-6: Evolution results training with the two most similar frames in a sequence of $10,30,60,100$, and 300 frames, compared to the previously obtained ones.

As it can be seen, although the results obtained with this approach are not as good as before, a search sequence of 30 frames is enough to outperform the median filter, and results close to the original are reached with a search sequence of 300 frames.

Original contribution 7-2 The use of two consecutive frames for training an EHW filter, as well as the search of optimal frames based on their similitude, are original contributions. Notice that other systems using consecutive frames (such as [Saeidi'05]) do not use these two frames in a training stage, but rather as inputs for the filter directly.

\footnotetext{
${ }^{6}$ Alternatively, the system may start evolving as soon as it receives two frames, changing its training set later in the evolution if better candidates are found, thus using the search time for the first stages of the evolution rather than wasting it. Furthermore, the system may use the partial result of the evolution as soon as a frame is received (as mentioned in $\$ 3.3 .5$ ), so the system will not be idle during the training stage. This has been implemented on the autonomous variation of the demo mentioned in $\$ 3.4 .5$ [Mora'14].

${ }^{7}$ There are 9 possible frame pairs in a search sequence of 10 frames.
} 


\subsubsection{Triggering evolution}

Once a solution for the autonomy of the system when obtaining a training data set has been found, a question remains: how to make the system aware that it requires to evolve?

Detecting when the system needs to be re-evolved (for example due to permanent faults in the SA or due to a change in the problem conditions) can be achieved by monitoring the filter performance, as is done in [Gallego' 12, Mora'14]. This can be done by comparing the filter input with its output (i.e., measuring the "quality gap" caused by the filter): a change in the conditions will likely reflect as the filter losing performance (thus making the gap smaller), having a higher effect due to the increased noise level (thus making the gap larger), or presenting an odd behavior due to a fault or a drastic change in the problem conditions (making the gap larger as well). Thus, when the gap deviates significantly from its previous values, it may be a signal that the system needs to be retrained for the new situation, and thus a new evolution needs to be triggered.

This monitoring can be done directly in the hardware described here, by using the filter input as reference and using the fitness computing unit to calculate the SAE between input and output. In [Gallego'12], a variation of the system is presented that allows redirecting the input directly to the fitness computing unit in order to simplify this process.

A modification of the demo described in $\$ 3.4 .5$ that implements all these features is described in [Mora'14].

\subsubsection{Conclusions}

In this section, a solution has been presented which enables evolvable video filtering systems to be fully autonomous; not only in the generation of filters, but also for obtaining training images and evaluating whether the evolution needs to be relaunched.

This solution is based on the correlation between consecutive frames; therefore, it will perform well as long as the target video sequence does not contain too much motion. Otherwise, longer video sequences may be used, and if that fails, it might be possible to use other techniques such as using some motion compensation, although that would increase the software complexity.

It should be noted that, since this approach relies on the randomness of the noise, it is not adequate for problems where the noise is not uniformly 
random and non-correlated between frames, for example, to filter dead pixels or dust in the camera lens, as in these cases the affected pixels exist in both the training input and training reference, and thus the system will not attempt to fix these pixels. Additionally, the range of application of this approach is limited to noise removal tasks; other tasks such as edge detection (as mentioned in $§ 3.3 .2$ ) fall out of its scope.

\subsection{EXTENDING THE PARADIGM TO DIFFERENT PROBLEMS}

Although one of the characteristics of the EHW approach is its adaptivity to different tasks, it is true that, with the exception of a few experiments validating the adaptivity of the system performed in $\$ 3.3 .2$, the only problem that has been experimentally assessed in this thesis has been the removal of salt and pepper noise, which is a substitutive noise that affects some of the pixels of the image while leaving the rest intact. However, it could be argued that this is a relatively simple task, since it consists in identifying which pixels are corrupt (which is easy in the case of salt and pepper noise since they are either extremely black or extremely white) and replacing them with a predicted value (which may entail a bigger complexity; the quality of the filter will be determined mainly by the accuracy of this task). Nevertheless, as it was said in $\$ 3.3 .2$, the adaptivity characteristic of this kind of system makes it potentially suited to a larger range of tasks, and some examples with different types of noise were provided.

However, since the main goal of this thesis is to ensure the generalizability of this system for its use in different tasks, it is important to formally assess whether or not it is suited for them. This section demonstrates the behavior of this system in different tasks, and studies possible ways to improve it in order to achieve a greater generalizability and independence from the task at hand. 


\subsubsection{Exploring applications other than substitutive noise filtering}

Throughout the elaboration of this thesis, several experiments outside of the field of substitutive noise filtering were performed in order to explore new potential applications of the system being studied. These new problems are described below.

\section{Description of the studied problems}

One task that has been explored is the removal of additive white Gaussian noise (AWGN), which is a type of noise that affects all pixels in the image by adding a normally distributed random value to each of them. This type of noise is usually found in camera sensors and many analog transmission channels, and is therefore a more realistic noise model than salt and pepper. However, this type of noise is specially difficult to remove with a window filter, since it requires correcting the value of every pixel and not just that of a few affected ones.

Another explored task has been the use of the evolvable filter as a hardware accelerator that approximates a given image processing algorithm. The algorithm used in this use case was a Sobel filter, which is an image operator that discriminates edges on an image; this operator is frequently used in edge detection applications. In this case, the task does not consist in removing a defect from an image, but in mimicking the effect of a known filter that could be computed in software but needs to be accelerated in hardware. Therefore, the training is performed using a clear image as the input and the result of applying a Sobel filter to that image in software as a reference.

Finally, another problem that has been analyzed is shade removal from text. The choice of this task is motivated by its role in applications such as optical character recognition, which often requires the characters to be "equalized" in terms of brightness and contrast. The training image set for this problem is elaborated by using a text sample as a reference and the same sample with locally altered contrast and brightness levels as input.

In addition to these tasks, two types of substitutive noise are studied as well in order to compare the results: salt and pepper noise (S\&P) and impulse noise, a similar type of noise where random pixels are replaced by random values uniformly distributed between black and white. The input and reference images used for each of these experiments are shown in Figure 7-7. 

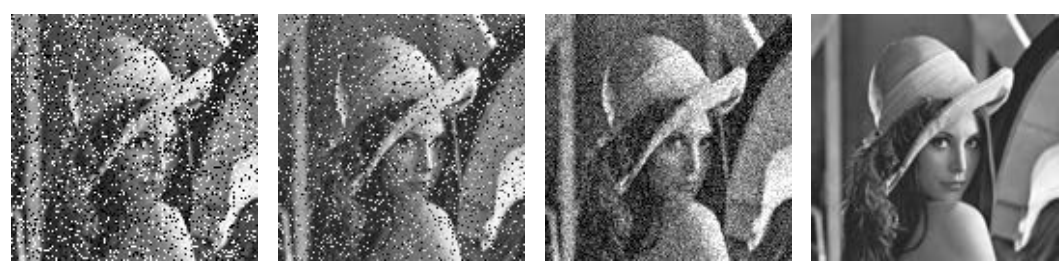

Lorem ipsum dolor sit a consectetur adipiscing $\epsilon$ do eiusmod tempor inci labore et dolore magna Ut enim ad minim venia nostrud exercitation ulla

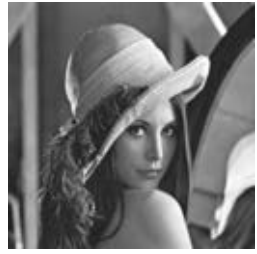

S\&P

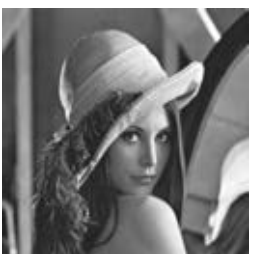

Impulse

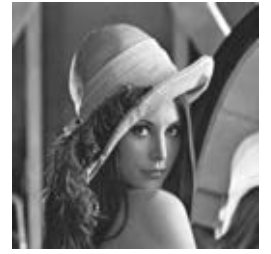

AWGN

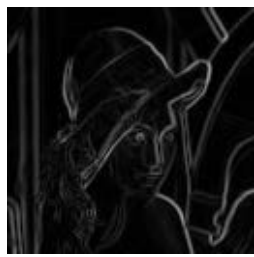

Sobel

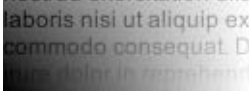

Lorem ipsum dolor sit a consectetur adipiscing $\epsilon$ do eiusmod tempor inci. labore et dolore magna Ut enim ad minim venia nostrud exercitation ulla laboris nisi ut aliquip ex commodo consequat. D irure dolor in reprehend

Shade

Figure 7-7: Input (top) and reference (bottom) images for different problems: $20 \%$ salt and pepper noise removal, $20 \%$ impulse noise removal, $10 \%$ additive white Gaussian noise removal, Sobel filter imitation, and shade removal

\section{Experimental results}

The current EHW system with $128 \times 8$ SAs in parallel has been applied to the five problems described above, using a similar EA to the one described in $\$ 6.1 .5$ : a 12-run (1+1)-EA with an exchange of worst with best every 1000 generations, for a total of 20000 generations (240000 candidate solutions evaluated).

Figure 7-8 shows the experimental results for these problems after 1000 evolutionary runs, in terms of fitness relative to the SAE between input and reference, with the outputs of some of the resulting filters displayed in Figure 7-9. As it can be seen, the results for AWGN are not as good as those for salt and pepper or impulse noise, consisting mostly in blurring the image, but nevertheless reduce the image SAE by $50 \%$. Sobel imitation and shade removal yield acceptable results despite of the different nature of those tasks, although the latter seems to perform poorly for very low contrast levels.

\section{Conclusions}

These experiments show that the described EHW system is not only suited for removal of substitutive noise, but also for several other different tasks in the field of image processing. However, in some cases (such as AWGN or shade removal), the results were not as good as one may expect. This is possibly due to the fact that these problems affect every pixel in the image, and thus may need a more complex filtering and more input data in order to obtain satisfactory results. 


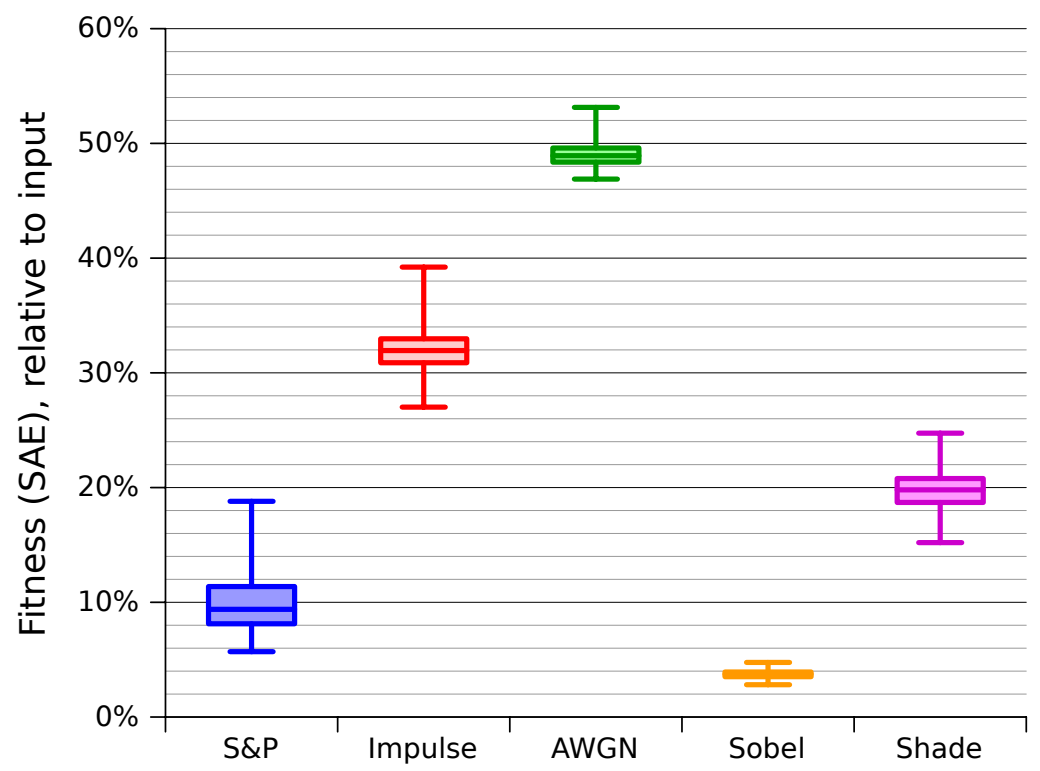

Figure 7-8: Evolution results for the five analyzed problems

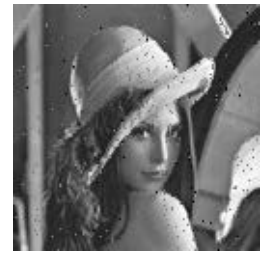

S\&P

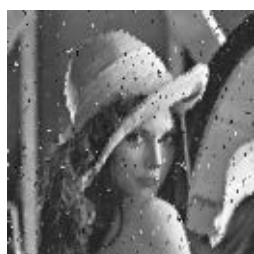

Impulse

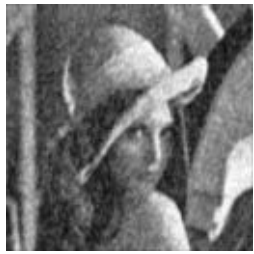

AWGN

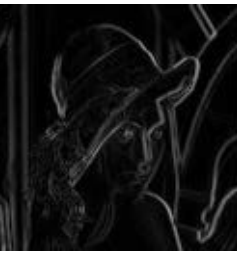

Sobel
Lorem ipsum dolor sit a consectetur adipiscing do eiusmod tempor inci labore et dolore magna Ut enim ad minim venia nostrud exercitation ulla laboris nisi ut aliquip ex commodo consequat. [ irure dolor in reprehene

Figure 7-9: Output of some of the evolved filters

One way to improve the capabilities of the processing system may be to improve the collection of PE functions initially described in Table 3-1. So far, these functions have proven useful for the task of salt and pepper image filtering; however, the PE architecture described in $\$ 5.1 .2$ would permit other PE function configurations which may be worth studying. It is also possible that different problems have different PE function requirements; for example, it is likely that tasks such as Sobel filter imitation make more use of linear functions (addition, difference, average...), whereas substitutive noise problems put more emphasis on the detection of atypical pixels, thus requiring nonlinear functions such as maximum and minimum. Therefore, exploring these potential new PE functions is necessary in order to elaborate a generalpurpose PE library that can successfully deal with different problems. 
On the other hand, the need for a greater range of input data motivates the exploration of larger window sizes. As it was said in $\S 5.1 .3$, the parameterization of the current EHW system makes it possible to implement window sizes of up to $5 \times 5$ pixels, which is an alternative worth exploring.

Finally, it may also be possible that certain tasks have greater precision requirements, making it necessary to increase the data width handled by the PEs from 8 bits to 16 or 32 . However, although this possibility has been taken into consideration and PEs have been parameterized in order to accept different data widths, evaluating these configurations has been considered out of the scope of this thesis.

\subsubsection{Generalization of the reconfiguration engine to accom- modate larger PBS libraries}

As it has been motivated above, tests that involve using larger sliding windows as well as extending the PE library need to be performed. However, in its current state, the reconfiguration peripheral as described in $\$ 5.1 .5$ has some limitations that make it unsuited for these tests and potential future extensions, so some design considerations need to be made prior to launching these tests. These design considerations not only aim at addressing the encountered problems, but also at generalizing the reconfiguration engine so that it can be suited for a wider range of applications.

\section{Motivation}

In $\$ 5.1 .5$, an IP interface built on top of the reconfiguration module proposed in $\$ 4.1$ was introduced with the goal of simplifying the control of that module for a specific use case: the fine-grain LUT-based SA architecture proposed in this thesis. This interface has the advantage of reducing the amount of configuration data needed for a single reconfiguration request to three 32-bit words, thus simplifying its control by the software application and reducing the time spent in sending data from the microprocessor to the peripheral. However, this approach presents a problem: while the underlying reconfiguration module is generalizable to many fine-grain DPR tasks, the described interface is not, constraining the IP to a very narrow range of applications.

The main problem of this IP is its limited addressing capacity for partial bitstreams. By using only 6 bits, a total of 64 PBS memory positions can be addressed. This is more than enough for the current system, as it is able to fit 
the 54 PBSs needed for the first and second stage of each of the 16 PE functions, 9 input selector configurations, and 2 output selector configurations. This would give a narrow margin of 10 extra PBS positions. However, increasing the number of input selectors to 25 (as required to implement a $5 \times 5$ input window) would raise the number of PBSs to 86, and increasing the number of PE functions to any value above 21 would overflow the capacity of the DPR engine as well. Additionally, applications such as the bypassable PEs proposed in $\$ 5.2 .4$ may require a single PE function to have a high number of variations (9 in this case). ${ }^{8}$ Therefore, it may be necessary to modify this DPR engine interface to accommodate more PBS positions, preferably in a configurable way.

Another concern is the use of memory. The described DPR engine is designed to operate using BRAM, which is a scarce resource whose use should be minimized. So far, this has not been a problem, since the PBSs are small in size and number, allowing to fit the entire PBS library within a single $4 \mathrm{~KB}$ BRAM block, which is the smallest size for a dual-port BRAM block. However, as this library grows, it may become interesting to reduce its memory footprint in order to avoid increasing the BRAM usage. Nevertheless, the PE structure proposed in $\$ 5.1 .2$ is highly compressible given that it uses the same LUT function for all eight LUTs on each stage, resulting in a PBS consisting of four repeated words. ${ }^{9}$ This opens the possibility of modifying the DPR engine to perform configurations that consist in repeating the same word or sequence of words multiple times ${ }^{10}$ (which is equivalent to having multiple configurable cells with identical PBS addresses stacked on top of each other); this strategy may be used to reduce the amount of memory required for the PBS library.

Finally, it should be noted that reconfiguration operations in the Virtex5 implementation of the current two-stage PE model always require reconfiguring a column of all first stages or all second stages (as it was shown in Figure 5-38 from $\$ 5.4 .3$ ), so the last bit of all PBS indexes will be the same (assuming they are ordered in pairs in the PBS memory). This would allow removing this bit from all indexes, making them shorter, and replacing

\footnotetext{
${ }^{8}$ In $\$ 5.2 .4$, this was solved by generating the configuration of a single 10-PE column on the fly rather than storing the 144 possible function variations in memory, which had important repercussions in the reconfiguration time.

${ }^{9}$ Each 32-bit configuration word contains data from two LUTs, as it was seen in $§ 5.1 .1$ (Table 5-1).

${ }^{10}$ It should be noted that this is a very simple compression method that requires the whole PBS to consist of repetition sequences of identical lengths, unlike other compression techniques such as RLE which allow varying lengths. One example of such technique is the multiple frame write mechanism supported by Xilinx FPGAs in which the same frame data can be written to multiple addresses, thus potentially reducing the bitstream size significantly.
} 
it with a single bit common to all of them. This may not seem like a great advantage, but given the current compactness of data in the configuration registers (see Table 5-5 from $\$ 5.1 .5$ ) it means being able to address twice as many PBS positions without adding an additional configuration register, with the overhead that transmitting said register would imply. ${ }^{11}$ Additionally, it simplifies the control from the software side, as it only requires writing the PBS index configuration words once for every two stages, since the second stage will use the same indexes as the first one, further reducing the number of transactions from the microprocessor to the peripheral. (This idea could be generalized to any DPR application where reconfigurable regions are fragmented into non-contiguous segments.)

All these considerations motivate the generalization of the interface of the DPR IP with the microprocessor.

\section{Generalizing the reconfiguration engine interface}

In order to address the requirements of the current design and others to come, a generalized fine-grain reconfiguration IP has been developed. This IP is built upon the generic reconfiguration module proposed in $\$ 4.1$ by adding a parameterized signal interface layer. The purpose of this interface is to map the data from the software-accessible IP registers to the control signals of the reconfiguration module in a customizable manner, so that reconfiguration engines tailored for different applications can be implemented by just setting certain parameters.

In order to simplify the addressing of individual PBSs (and thus shorten the addressing information that needs to be transmitted from processor to peripheral), the address space of the PBS memory has been logically partitioned into multiple positions marking the beginning of a PBS, with three hierarchical indexes associated to each position: a major index that can be specified individually for each reconfigurable cell, an optional middle index that is chosen at design time for each cell, and an optional minor index that is common to all cells in a column. The minor index is useful when there are several types of reconfigurable columns, each with its own version of the PBS library (e.g., stage 1 and stage 2 LUT columns in the LUT-based PE design described in $\$ 5.1$, or designs where SLICEL and SLICEM columns require

\footnotetext{
${ }^{11}$ Given the high speed of the current reconfiguration engine (about $0.5 \mu \mathrm{s}$ ) and the relatively large latency of transmitting a single word through the system bus (about $0.1 \mu \mathrm{s}$ ), the current bottleneck in the reconfiguration process is suspected to be in the transmission of these words rather than the ICAP configuration itself.
} 
different PBSs for the same functionality [Gallego'14]). Similarly, the middle index may specify different types of cell within each column. ${ }^{12}$

Figure 7-10 shows an example of this hierarchical indexing for a 10-bit address with a 5-bit major index $(\mathrm{X}), 1$-bit middle index $(\mathrm{Y})$, and 2-bit minor index (Z); the effective PBS initial address will be the result of concatenating these indexes and setting the remaining 2 bits to zero.

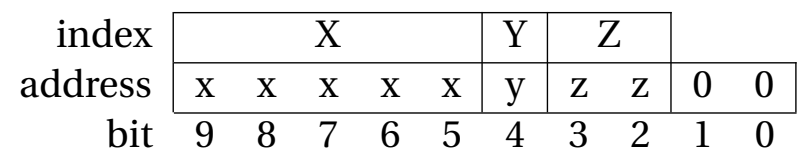

Figure 7-10: Example mapping from hierarchical indexes to PBS address: $(\mathrm{X})$ major index, $(\mathrm{Y})$ middle index, $(\mathrm{Z})$ minor index

Major indexes are supplied through the microprocessor-accessible IP configuration registers. The first register is reserved for the transmission of the initial frame address, the number of frames to reconfigure, and the minor index common to all cells, all of which are specific to each reconfigurable column and can therefore be considered as the "column specification". The remaining registers are divided into several sub-registers, each intended to hold a major index; which sub-register corresponds to each cell can be configured at design time. As an example, a register layout for an implementation with 6-bit major indexes, 1-bit minor index, and 7-bit frame count can be seen in Table 7-1, with the major index sub-registers labeled A to J.

\begin{tabular}{c||c|c|c|c|c|c|c|} 
Bits & 31 & 30 & $29 \ldots 24$ & $23 \ldots 18$ & $17 \ldots 12$ & $11 \ldots 6$ & $5 \ldots 0$ \\
\hline \hline Register 0 & Z & \multicolumn{2}{|c|}{ frames } & \multicolumn{4}{|c|}{ frame address } \\
\hline Register 1 & - & E & D & C & B & A \\
\hline Register 2 & - & J & I & H & G & F \\
\hline
\end{tabular}

Table 7-1: Example register description

Overall, the following parameters can be configured: number of softwareaccessible IP registers, number of reconfigurable cells in which reconfigurable regions are vertically partitioned, height of each cell, sub-register corresponding to each cell, middle index of each cell, address width (memory size), and widths of major index, middle index, minor index, frame address, and frame count.

\footnotetext{
${ }^{12}$ This may be useful for example in the PE layout for 7 Series FPGAs described in $\$ 5.4 .3$, which has two "twin" cells stacked on top of each other, one for each PE stage (Figure 5-38, right).
} 
Lastly, it should be mentioned that, since the interface layer described here is simply a mapping from registers to control signals, it has no impact in the resource usage: it is implemented as a series of nets that do not use any logic. ${ }^{13}$

Original contribution 7-3 A highly parameterized fine-grain reconfiguration engine supporting compact and efficient PBS addressing, varying target applications, and compression of PBSs consisting of repeating data. This engine employs an original indexing scheme for $P B S$ addressing in order to minimize data transactions.

\section{Results}

The new generalized reconfiguration IP core has been used in the SA-based EHW system, configured with 6-bit major indexes and a 1-bit minor index. This allows having up to 64 different PE/selector functions, enough for up to $53 \mathrm{PE}$ functions (for a $3 \times 3$ window), or $37 \mathrm{PE}$ functions if a $5 \times 5$ window is used. The 1-bit minor index allows for two versions of each PBS, which will be used to host the two stages of the PE. These $64 \times 2$ PBSs still fit on a single 4 KB RAMB36 primitive, and thus do not increase the resource usage in terms of BRAM.

As a side effect, this improved reconfiguration IP core simplifies the reconfiguration of designs based on two-stage PEs such as the one at hand: since the major indexes for both stages are the same and only the minor index changes, these major indexes only need to be transmitted before reconfiguring the first stage and not the second one, thus saving data transactions between microprocessor and peripheral. It has been experimentally observed that this reduces the reconfiguration time from 5.2 to $3.7 \mu \mathrm{s}$. Under these new conditions, the candidate solution evaluation speed as calculated in $\$ 6.2$ could go up to 187000 evaluations per second if 16 SAs were used in parallel, reducing the total time of a 192000 -candidate evolution to $\mathbf{1 . 0 3}$ seconds. ${ }^{14}$

This generalized reconfiguration engine could also be useful for other applications that require a high number of PBSs, such as the bypassable PEs proposed in $\$ 5.2 .4$. This application requires 9 times more PBSs, since

\footnotetext{
${ }^{13}$ The only exception to this is the conversion from number of frames to number of words, which involves multiplying by the frame size. This is done to reduce the size of this field.

${ }^{14}$ However, implementing a system with these many arrays has proven to be impractical (the whole embedded system design required more than 13 hours to synthesize on the employed computer, which is an indicator that the resulting system is reaching the limits of feasibility of its implementation due to placement and routing complexity). For consistency with previously obtained evolution results, it has been decided to stick to the previous setup of 12 SAs.
} 
stage 2 of each PE can work in 9 different ways for every possible function depending on the bypass configuration for east and south outputs. Therefore, the reconfiguration engine for this system would require a capacity for at least 180 PBSs, which can be easily achieved by using a different configuration of the proposed reconfiguration IP core; additionally, as it was suggested before, a single reconfigurable cell may be replaced by four identical cells that use the same PBS given the homogeneity of the LUT configuration across the eight LUTs within the same PE stage, effectively compressing this PBS to a quarter of its size, allowing the whole 180-PBS library to fit on a single 4 KB RAMB36 primitive.

This parameterization of the reconfiguration engine may also become useful in future implementations of arrays of PEs with different data widths, since these would only require to change the height of each reconfigurable cell (or the number of repeated cells if compression is used); however, evaluating these setups is out of the scope of this thesis.

\subsubsection{Increasing the size of the sliding window}

The first experiment that has been performed has consisted in evaluating the performance of the EHW system when using a $5 \times 5 \mathrm{px}$ window. The EA is identical to the previous one, with the genes controlling the input selectors being able to take 25 different values rather than 9 .

The evolution results after 1000 evolutionary runs under these parameters are shown in Figure 7-11, compared to the ones obtained with a $3 \times 3$ window. Fitness values are displayed relative to the median value that was obtained in each problem with the $3 \times 3$ window. As it can be seen, the new window size does not provide an improvement on the evolution results: SAE increases by $7 \%$ for salt and pepper noise and by $3.5 \%$ for Sobel imitation, and yields nearly the same results for the other three problems (with less than $0.5 \%$ improvement). Specifically, the only problem that was shown to improve with statistical significance ( $p=0.004$ for a Wilcoxon-Mann-Whitney test) was AWGN, which only reduced the median SAE by $0.25 \%$.

These bad results could be explained by the fact that the new input window leads to an excessively big search space, making the EA unable to converge to a good choice of pixels from the window. Additionally, the $8 \times 8$ SA may be too small to take full advantage of these window sizes, since it only has 16 inputs, so not all pixels from this $5 \times 5$ window will be used. Therefore, the EA should be improved in order to take full advantage of this window size, for example by modifying the mutation mechanism for input selector genes, but this has been 


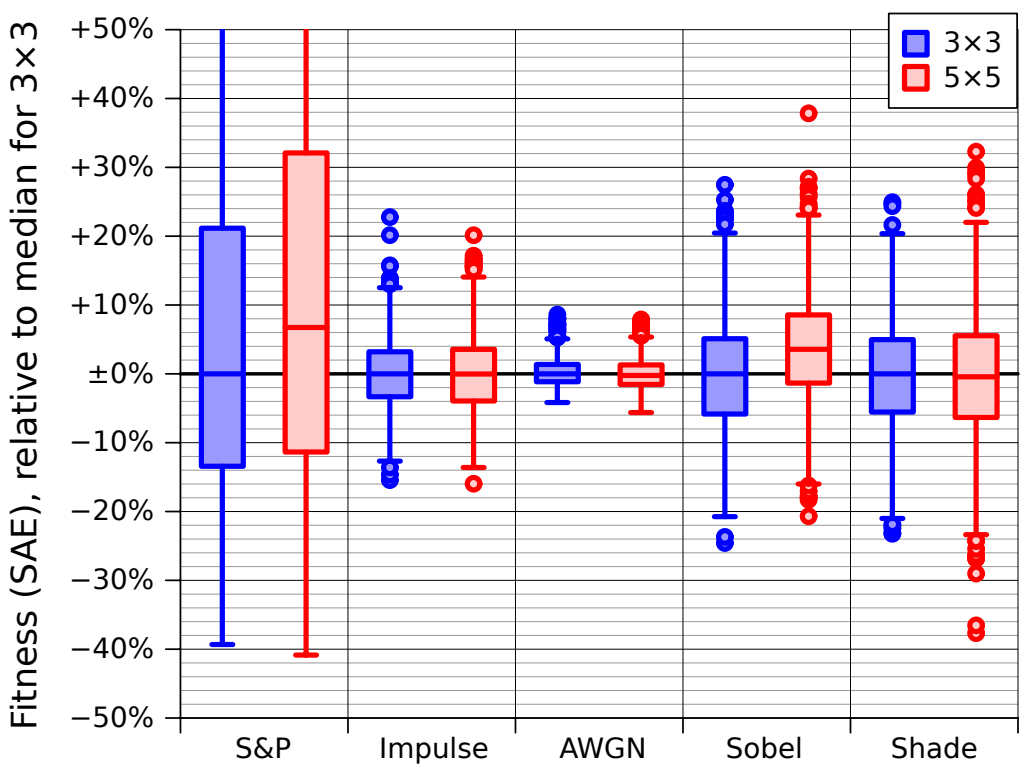

Figure 7-11: Comparison of evolution results with $3 \times 3$ and $5 \times 5$ windows

considered out of the scope of this thesis. Nevertheless, it should be noted that the system with a $5 \times 5$ window is a superset of one with a $3 \times 3$ window, and thus it is possible to perform a $3 \times 3$ window filtering without changing the hardware by simply using a subset of the input window in the EA.

\subsubsection{Improving the PE library}

Another possibility that has been explored is the improvement of the PE function library. So far, the set of functions implemented by the PEs in this thesis has always been the same. This set was defined in [Salvador'11a], and is a subset of the set of 34 functions described in [Sekanina'04] obtained by choosing the functions that appeared most frequently in the evolved solutions. This reduced set of functions was partly motivated by the need of having a PE library with a small memory footprint, as well as having to synthesize each PE individually. However, the LUT-based PE architecture described in $\$ 5.1 .2$ opens the possibility of implementing a wide range of functions in the same hardware without having to synthesize each function individually and with a very small memory footprint.

Prior to this work, some research effort into expanding the PE function library was made in [Niño'15]; however, not all of the functions proposed in said work can be accurately implemented in the current PE architecture, and 
some of them required an additional parameter that needed to be evolved as well, significantly deviating from the proposed EHW architecture.

\section{Potential new PE functions}

In order to explore the effect of altering the PE function library, a series of possible PE functions has been designed, with the restriction that all functions must follow the scheme proposed in $\$ 5.1 .2$ (Figure 5-4): ${ }^{15}$

- A stage 1 adder/comparator which may add none, one, or both inputs or their bitwise complement.

- A stage 2 selector which yields one of the inputs, the result of the adder, said result divided by 2 , a constant value of 0 , or the complement of any of those, possibly conditioned by the carry output of the adder.

This restriction not only ensures that the PEs can be implemented in the current PE architecture, but also that the LUT content will be the same for all 8 LUTs in each stage, thus simplifying the generation of PBSs and allowing their compression as explained in $\$ 7.2 .2$.

The list of 44 proposed PE functions is shown in Table 7-2. This list extends the original one in $\$ 3.1$ with more constants ( 0 and 128), more arithmetic functions covering different behaviors on overflow, some conditional functions (denoted as "condition ? result-when-true : result-when-false") that yield 0 or 255 or the value of one input conditioned to the value of the other, and two functions that approximate the absolute value of the difference. ${ }^{16}$ Additionally, a Matlab script was developed that finds the closest approximation of arbitrary functions by brute force evaluation of the 2100 possible combinations. Despite of relying on brute force, it finds a solution in a few seconds.

Original contribution 7-4 A list of PE functions that can be implemented in terms of LUT functions for the proposed two-stage PE architecture.

\footnotetext{
${ }^{15}$ This excludes some possible non-arithmetic functions such as bitwise AND or addition of an arbitrary constant that could be implemented in the current PE architecture.

${ }^{16}$ The function $|N-W|$ cannot be exactly calculated in the current PE architecture, since it requires two comparators/adders and the PEs only have one. Nevertheless, approximations that yield the correct value when $N \geq W$ and the value minus 1 when $N<W$ (or vice versa) can be implemented in this architecture.
} 


\begin{tabular}{cc|cc|cc}
$\#$ & Equation & $\#$ & Equation & $\#$ & Equation \\
\hline 0 & $N+W \bmod 256$ & 16 & 0 & 31 & $\min (N-W+256,255)$ \\
1 & $2 N \bmod 256$ & 17 & 128 & 32 & $\min (W-N+256,255)$ \\
2 & $2 W \bmod 256$ & 18 & $255-N$ & 33 & $N-W \bmod 256$ \\
3 & $\min (N+W, 255)$ & 19 & $255-W$ & 34 & $W-N \bmod 256$ \\
4 & $\min (2 N, 255)$ & 20 & $\max (2 N-256,0)$ & 35 & $(N \geq 128) ? 255: 0$ \\
5 & $\min (2 W, 255)$ & 21 & $\max (2 W-256,0)$ & 36 & $(W \geq 128) ? 255: 0$ \\
6 & $\left\lfloor\frac{N+W}{2}\right\rfloor$ & 22 & $\min (N, 255-N)$ & 37 & $(N \geq 128) ? W: 0$ \\
7 & 255 & 23 & $\min (W, 255-W)$ & 38 & $(W \geq 128) ? N: 0$ \\
8 & $\left\lfloor\frac{N}{2}\right\rfloor$ & 24 & $\left\lfloor\frac{N}{2}+128\right\rfloor$ & 39 & $(N \geq 128) ? W: 255-W$ \\
9 & $\left\lfloor\frac{W}{2}\right\rfloor$ & 25 & $\left\lfloor\frac{W}{2}+128\right\rfloor$ & 40 & $(W \geq 128) ? N: 255-N$ \\
10 & $N$ & 26 & $\max (N+W-256,0)$ & 41 & $(N \geq W) ? 255: 0$ \\
11 & $W$ & 27 & $\left\lfloor\frac{N-W}{2}+128\right\rfloor$ & 42 & $(W \geq N) ? 255: 0$ \\
12 & $\max (N, W)$ & 28 & $\left\lfloor\frac{W-N}{2}+128\right\rfloor$ & 43 & $(N+W \geq 256) ? 255: 0$ \\
13 & $\min (N, W)$ & 29 & $\left\lfloor\left|N-W+\frac{1}{2}\right|\right\rfloor$ & & \\
14 & $\max (N-W, 0)$ & 30 & $\left.\left\lfloor\mid W-N+\frac{1}{2}\right\rfloor\right\rfloor$ & & \\
15 & $\max (W-N, 0)$ & & & &
\end{tabular}

Table 7-2: List of proposed PE functions, consisting of the 16 original functions (0-15) and 28 new ones (16-43)

Original contribution 7-5 A brute-force Matlab script to obtain PE configurations that match or approximate arbitrary functions.

Results after evolving with this new PE function library are shown in Figure 7-12. As before, results for each problem are represented relatively to the median value obtained with the original library for that problem.

As it can be seen, with the exception of the S\&P problem, which achieves a reduction of $3 \%$ in the SAE with this extended library, all other problems have their SAE incremented between $3 \%$ (for shade removal) and $18 \%$ (for impulse noise), despite of the new library being a superset of the old one and therefore being able to implement the same solutions and more. This is most likely a consequence of the search space becoming excessively large with these many functions. Therefore, in order to avoid this, the library should be kept at a moderate size (such as the previous 16 element size); finding out 


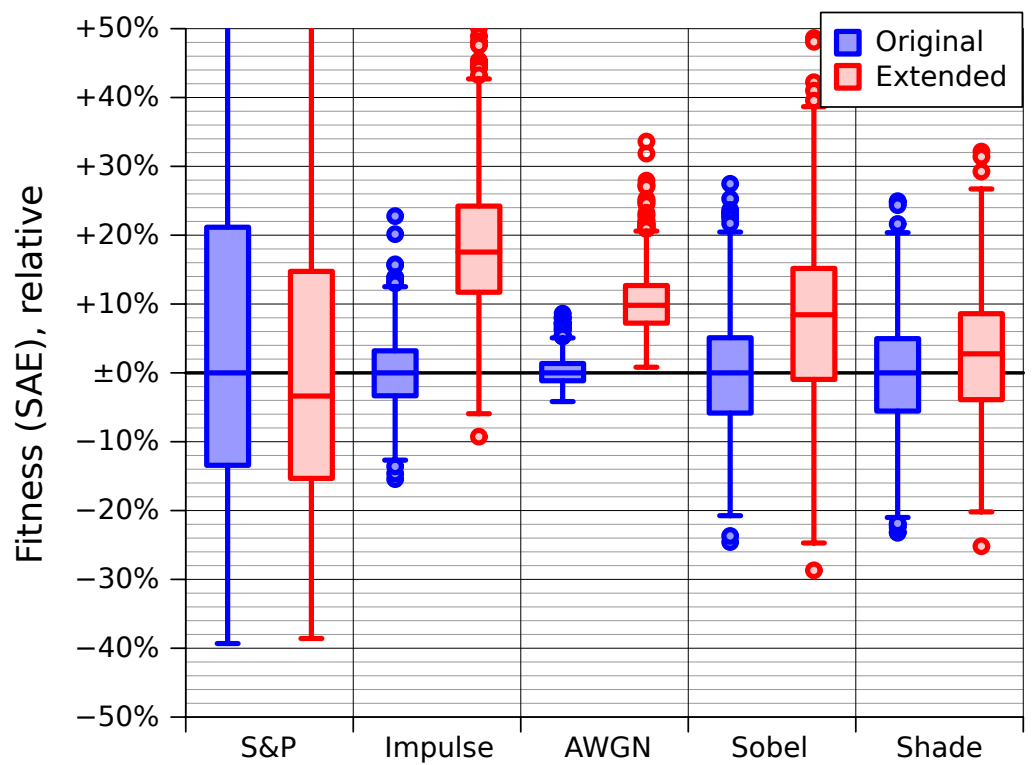

Figure 7-12: Evolution results with the extended $44 \mathrm{PE}$ function library, compared with the original one

which functions are best suited will require a more detailed analysis that will be elaborated below.

Alternatively, an EA could be developed which not only evolves the SA configuration but also the set of functions used for this, adding or removing functions from the global 44 element library in order to select the most appropriate ones; however, this would require a careful analysis that has been regarded as out of the scope of this thesis.

\section{Systematic analysis of the impact of each function}

In order to evaluate the suitability of each of these PE functions for each problem, the following experiment has been devised: first, a random 16function library is generated by randomly selecting functions from the set of 44 proposed functions; ${ }^{17}$ then, the EA is run using that function library; ${ }^{18}$ finally, the obtained fitness is annotated together with the 16 used functions. In order to obtain statistically meaningful results, this process is repeated 10000 times for each of the five problems. Once all 10000 runs have been performed, a

\footnotetext{
${ }^{17}$ Some of these 16 functions may be repeated.

${ }^{18}$ Since there is no guarantee that pass-through functions (\#10 and \#11) are among the 16 chosen ones, the EA population is initialized with a random configuration rather than a passthrough one as it is done in the rest of experiments.
} 
figure of merit is calculated for each function by averaging all of the fitness values that were obtained using this function.

Table 7-3 shows the rank of each function for each of the problems, with 1 being the function that yielded the lowest average fitness and 44 the one with the highest. Additionally, the average of these five ranks for each function is shown, as well as the rank of said average, in order to make an approximate idea of the overall performance of each function.

As it can be seen, the functions yielding the best results (marked in bold in the table) vary considerably depending on the problem: for example, positive and negative average functions (\#6, \#27, \#28) are very beneficial to AWGN, Sobel, and shade but perform poorly for S\&P and impulse. Similarly, modulo256 functions (\#0, \#33, \#34) perform nicely on S\&P but not so well on the rest. Some functions, such as maximum and minimum (\#12,\#13) are beneficial for all problems, and others such as constants (\#7,\#16,\#17) and multiplication and division by 2 (\#1, \#2, \#4, \#5, \#8, \#9, \#24, \#25) seem to perform poorly in general.

Therefore, it can be concluded that, while the optimal PE function library will depend on the problem to solve—as it was suggested in [Sekanina'04]—, it might be possible to find a generalized function library that yields satisfactory results for a wide range of problems.

Original contribution 7-6 A methodology for optimizing the PE function library and comparing the contribution of individual functions to the overall fitness. This method is likely more robust than the selection of most frequently occurring functions from [Sekanina'04] performed in [Salvador'11a] as it covers the whole evolution process.

\section{Analysis of results of a library optimized for S\&P}

Using the results from Table 7-3, a 16-function library optimized for the S\&P problem was elaborated. This library contains the two $W$ and $N$ identity functions (\#11 and \#10), ${ }^{19}$ as well as the 14 functions other than these that ranked highest for the S\&P problem (\#12, \#33, \#0, \#13, \#34, \#29, \#14, \#31, \#3, \#30, \#15, \#40, \#32, \#26).

Figure 7-13 shows the results of evolving with this optimized library. As it can be seen, this choice of PE functions considerably improves the evolution

\footnotetext{
${ }^{19}$ Given that the EA always initializes the first chromosome as a pass-through filter, it has been decided to always make the $W$ and $N$ identity functions (\#11 and \#10) available regardless of their performance. Specifically, \#11 has been included in this 16-function optimized library regardless of being ranked in 18th position for the S\&P problem.
} 


\begin{tabular}{|c|c|c|c|c|c|c|c|}
\hline \# & S\&P & $\operatorname{Imp}$ & AWGN & Sobel & Shade & Average & Rank \\
\hline 0 & 3 & 12 & 8 & 21 & 28 & 14.4 & 13 \\
\hline 1 & 32 & 36 & 28 & 41 & 37 & 34.8 & 36 \\
\hline 2 & 22 & 41 & 34 & 37 & 43 & 35.4 & 40 \\
\hline 3 & 10 & 7 & 13 & 19 & 13 & 12.4 & 8 \\
\hline 4 & 31 & 39 & 39 & 33 & 42 & 36.8 & 42 \\
\hline 5 & 24 & 26 & 38 & 30 & 38 & 31.2 & 33 \\
\hline 6 & 43 & 38 & 1 & 3 & 1 & 17.2 & 17 \\
\hline 7 & 26 & 28 & 29 & 32 & 41 & 31.2 & 33 \\
\hline 8 & 36 & 31 & 11 & 18 & 24 & 24.0 & 24 \\
\hline 9 & 38 & 33 & 17 & 15 & 20 & 24.6 & 25 \\
\hline 10 & 7 & 15 & 6 & 9 & 12 & 9.8 & 4 \\
\hline 11 & 18 & 14 & 10 & 12 & 14 & 13.6 & 11 \\
\hline 12 & 1 & 1 & 4 & 10 & 2 & 3.6 & 1 \\
\hline 13 & 4 & 2 & 5 & 6 & 10 & 5.4 & 2 \\
\hline 14 & 8 & 3 & 19 & 17 & 5 & 10.4 & 5 \\
\hline 15 & 12 & 6 & 20 & 14 & 11 & 12.6 & 9 \\
\hline 16 & 34 & 40 & 27 & 35 & 40 & 35.2 & 39 \\
\hline 17 & 44 & 44 & 42 & 39 & 44 & 42.6 & 44 \\
\hline 18 & 16 & 19 & 7 & 25 & 18 & 17.0 & 15 \\
\hline 19 & 20 & 17 & 18 & 26 & 26 & 21.4 & 20 \\
\hline 20 & 33 & 32 & 40 & 36 & 39 & 36.0 & 41 \\
\hline 21 & 30 & 25 & 35 & 34 & 33 & 31.4 & 35 \\
\hline 22 & 23 & 23 & 26 & 5 & 32 & 21.8 & 21 \\
\hline 23 & 25 & 21 & 24 & 11 & 29 & 22.0 & 22 \\
\hline 24 & 37 & 34 & 9 & 22 & 21 & 24.6 & 25 \\
\hline 25 & 39 & 37 & 16 & 23 & 22 & 27.4 & 30 \\
\hline 26 & 15 & 4 & 21 & 24 & 15 & 15.8 & 14 \\
\hline 27 & 42 & 43 & 3 & 8 & 4 & 20.0 & 19 \\
\hline 28 & 41 & 35 & 2 & 4 & 3 & 17.0 & 15 \\
\hline 29 & 6 & 9 & 25 & 2 & 17 & 11.8 & 6 \\
\hline 30 & 11 & 10 & 23 & 1 & 19 & 12.8 & 10 \\
\hline 31 & 9 & 5 & 12 & 7 & 7 & 8.0 & $\mathbf{3}$ \\
\hline 32 & 14 & 8 & 15 & 16 & 6 & 11.8 & 6 \\
\hline 33 & 2 & 16 & 14 & 13 & 25 & 14.0 & 12 \\
\hline 34 & 5 & 18 & 22 & 20 & 23 & 17.6 & 18 \\
\hline 35 & 40 & 29 & 43 & 40 & 36 & 37.6 & 43 \\
\hline 36 & 35 & 27 & 36 & 42 & 35 & 35.0 & 38 \\
\hline 37 & 27 & 20 & 31 & 31 & 31 & 28.0 & 31 \\
\hline 38 & 17 & 13 & 41 & 29 & 27 & 25.4 & 27 \\
\hline 39 & 28 & 22 & 33 & 28 & 34 & 29.0 & 32 \\
\hline 40 & 13 & 11 & 32 & 27 & 30 & 22.6 & 23 \\
\hline 41 & 19 & 24 & 37 & 44 & 8 & 26.4 & 29 \\
\hline 42 & 21 & 30 & 30 & 38 & 9 & 25.6 & 28 \\
\hline 43 & 29 & 42 & 44 & 43 & 16 & 34.8 & 36 \\
\hline
\end{tabular}

Table 7-3: Rank of each PE function for each problem and overall rank 
results for the S\&P problem, reducing its median fitness by $\mathbf{3 1 \%}$. However, since this library has been optimized specifically for that problem, it does not work so well with the other problems: impulse noise and shade removal yield approximately the same results as before, whereas AWGN and Sobel imitation yield considerably worse fitness values, $24 \%$ and $40 \%$ higher respectively. This indicates that the optimized library has been overfit for a specific problemS\&P noise filtering-and therefore is not generalizable to other problems.

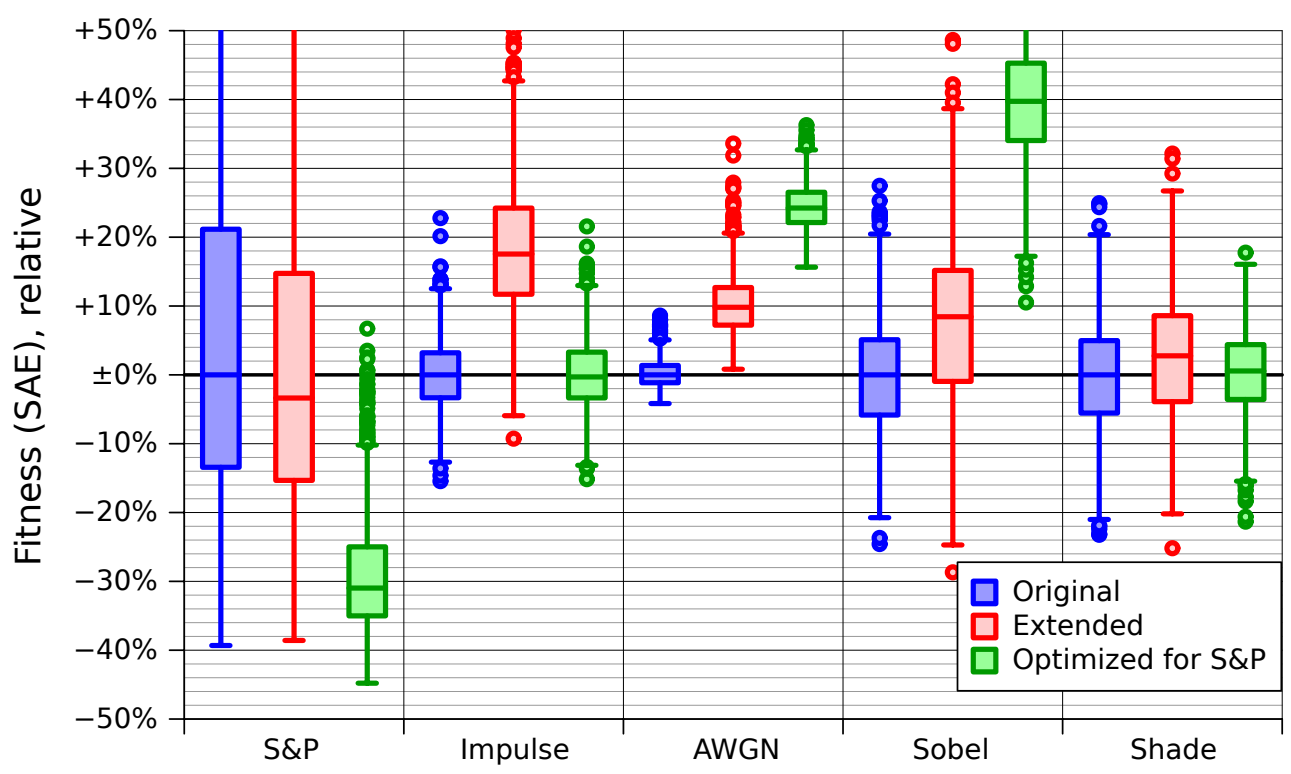

Figure 7-13: Evolution results with the PE function library optimized for S\&P, compared with the previous ones

\section{Optimizing the PE library for each problem}

In order to obtain similarly good results for the rest of the problems, different PE function libraries have been developed, each with the best 16 functions for each problem (always including $N$ and $W$ identity functions), following the ranks in Table 7-3.

The results for each problem using a PE function library optimized for that problem are shown in Figure 7-14. As it can be seen, in this case the improvement applies to almost all problems: in addition to the aforementioned $31 \%$ reduction of SAE for S\&P, there is a $1.5 \%$ reduction for impulse noise, $12 \%$ for Sobel imitation, and $13 \%$ for shade removal. The only exception is AWGN, which does not present any improvement-in fact, its SAE increases slightly $(+0.3 \%)$. 


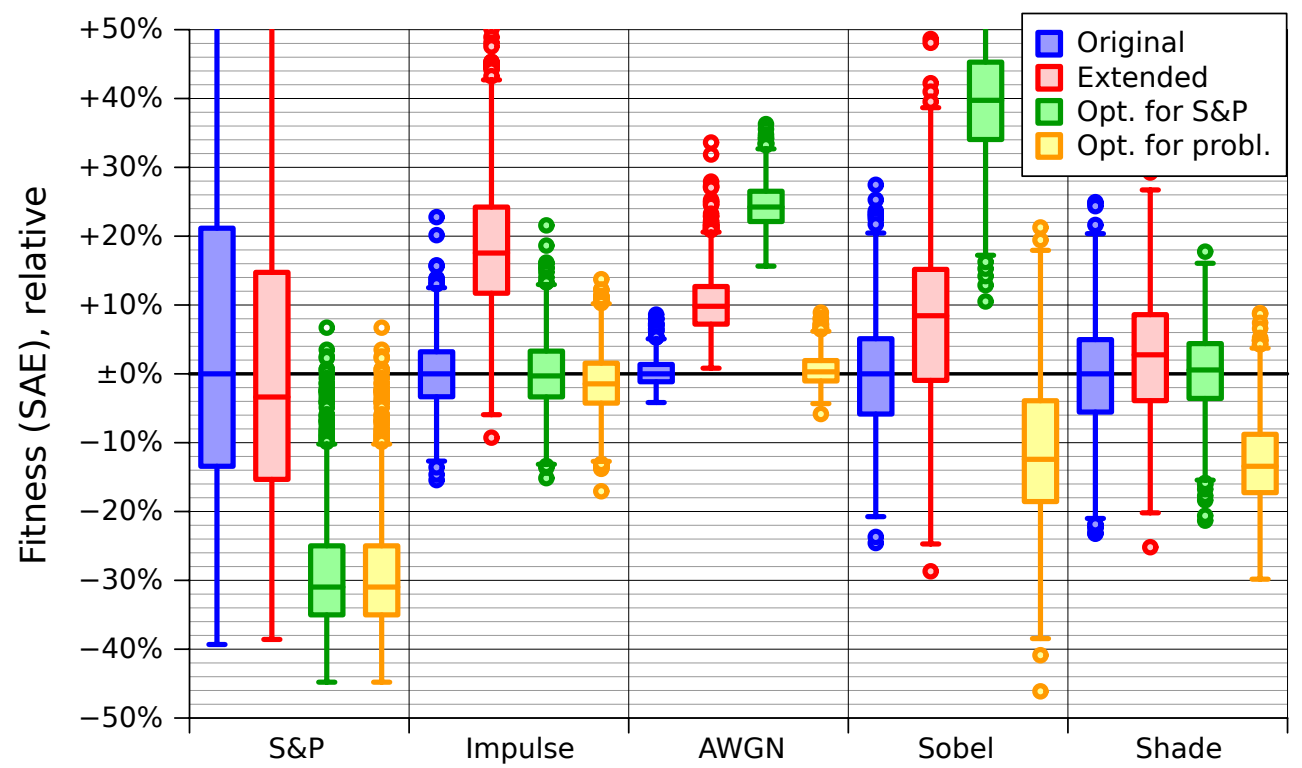

Figure 7-14: Evolution results with a PE library optimized for each problem

However, this approach has an important drawback: in this case, the system needs to be preloaded with a library specific to the problem to solve, and therefore requires previous knowledge of the problem. It is, therefore, not a real "agnostic" adaptive system able to process arbitrary problems, but only a very specific one or a narrow range of them. Therefore, this is not a satisfactory solution for this thesis: an ideal solution should be able to address arbitrary problems with a single library.

Nevertheless, these results are very interesting, as they show that the choice of a specific set of PE functions greatly affects the outcome, suggesting that there is room for improvement in the PE function library.

\section{Design of a general-purpose improved PE library}

In order to provide a generalized solution, an attempt has been made at designing a compromise PE function library that yields satisfactory results for all of the problems in scope. As it can be seen from Table 7-3, different problems evolve better with different categories of functions. For example, S\&P performs very well with modulo 256 addition and subtraction (\#0, \#33, \#34), but these functions yield poor results for the rest of problems; average functions (\#6, \#27, \#28) are greatly beneficial for AWGN, Sobel, and shade removal but detrimental for S\&P and impulse. Therefore, a general purpose library should compromise 
among these categories, picking the most globally beneficial ones and avoiding the ones that perform worst.

A PE function library is proposed in Table 7-4, along with the corresponding function indexes from Table 7-2. This library includes $W$ and $N$ identity functions, maximum and minimum (which were observed to provide the best overall performance), addition and subtraction with different overflow behaviors, and halved addition and subtraction.

\begin{tabular}{cc|cc}
$\#$ & Equation & $\#$ & Equation \\
\hline 11 & $W$ & 12 & $\max (N, W)$ \\
10 & $N$ & 13 & $\min (N, W)$ \\
26 & $\max (N+W-256,0)$ & 0 & $N+W \bmod 256$ \\
14 & $\max (N-W, 0)$ & 33 & $N-W \bmod 256$ \\
15 & $\max (W-N, 0)$ & 34 & $W-N \bmod 256$ \\
3 & $\min (N+W, 255)$ & 6 & $\left\lfloor\frac{N+W}{2}\right\rfloor$ \\
31 & $\min (N-W+256,255)$ & 27 & $\left\lfloor\frac{N-W}{2}+128\right\rfloor$ \\
32 & $\min (W-N+256,255)$ & 28 & $\left\lfloor\frac{W-N}{2}+128\right\rfloor$
\end{tabular}

Table 7-4: Proposed PE function library

The results for this proposed library are shown in Figure 7-15. As it can be seen, evolution results achieved with this new library are greatly beneficial for S\&P, decreasing the obtained SAE by $\mathbf{1 5 \%}$, and to a lesser degree to shade removal (4\%); all without an excessive negative impact on the other problems, which have their SAE increased between $2 \%$ and $4 \%$. Therefore, it could be considered that changing to this library is a good solution overall, although this may not be the case if problems such as impulse noise or AWGN are expected.

Original contribution 7-7 The proposal of a 16 PE function library that noticeably improves the evolutionary results of several problems. 


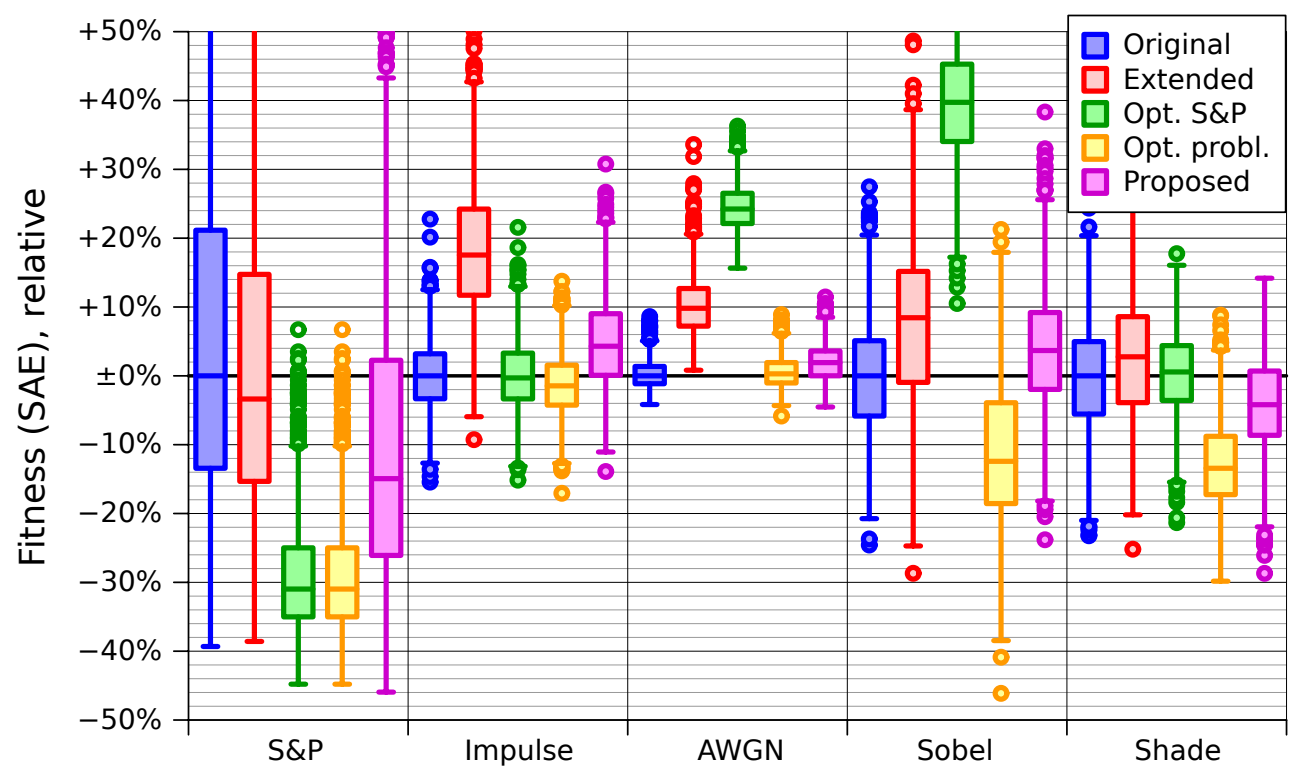

Figure 7-15: Evolution results with the proposed PE library

\subsubsection{Conclusions}

In this section, a methodology for designing new PE functions for the LUT-based PE architecture proposed in $\$ 5.1 .2$ has been described. This methodology leads to some interesting properties on the resulting PEs such as compressibility of the PBS and scalability to different data widths. Additionally, a methodology for evaluating the performance of these functions for different problems has been established, and a potentially better function library has been proposed based on it. This methodology only studies the effect of individual functions, and thus could be improved in a future by studying the interactions between different functions, analyzing which functions are redundant and which functions complement each other.

Furthermore, other possibilities such as different window sizes have been studied, which open the door to future development in the line of improving the EA to take advantage of these sizes.

Finally, a less conceptual and more technical improvement introduced in this section has been the generalization of the reconfiguration engine, which simplifies the generalization of the described system to different platforms and for different purposes. This opens the door to future research lines involving different PE shapes, such as the use of different data widths (e.g., 16 or 32 bits) which would permit a more precise processing. In addition, these improvements have also impacted in the reconfiguration process, which 
is now noticeably more efficient and simpler to control, potentially increasing the evaluation speed to $\mathbf{1 8 7 0 0 0}$ candidate solutions per second and reducing the evolution time to one second if the number of SAs is accordingly increased.

All these results show that this system is suitable for a wide range of problems and can be used in numerous applications. In some cases, however, it might be desirable to perform a prior analysis of the problem in order to obtain an optimized PE library for it.

\subsection{EXAMPLE DATAFLOW-BASED APPLICATION: STREAM VIDEO PROCESSING SYSTEM}

One of the main goals that have been pursued in this thesis is that the elaborated tools and methodologies can find practical applications in real world projects. This has been most prominently shown in $\$ 5.4$, where efforts have been made in order to improve the compatibility of the system with preexisting and potential applications by adopting more up-to-date technologies as well as a dataflow design approach.

In order to demonstrate the versatility of modules developed following this methodology, an example real-time video processing application was developed using this dataflow-oriented approach. Although not of much academic relevance, the development of this application has been considered important to show the feasibility of using the described system within larger projects. Since this is a mere demonstrative application which makes use of blocks that have been already studied, no experimental data assessing its properties is provided. 


\subsubsection{Description of the video processing system}

The premise for the development of this example application has been the implementation of an edge/corner detection system that can be used in combination with noisy images. Typically, edge and corner detection filters (Sobel, Harris, FAST...) are highly sensitive to noise [Rashmi'13, Dey'12, Rosten'06], since corrupted pixels result in abrupt differences in the image brightness which may be mistaken with the border of a shape. Therefore, these applications typically include a previous stage that reduces the noise of the video input, either with a specialized noise filter or with a simple Gaussian blurring filter.

In this example, this noise filtering is performed by the stream-oriented SAbased configurable video filter proposed in $\$ 5.4$. As an alternative, a Gaussian blurring filter is also provided in order to show the difference between these two filtering approaches. The filtered video stream is then processed by either an edge or a corner detection filter, which are considered system invariants. ${ }^{20}$ The configuration for the SA-based filter is obtained using a BRAM-oriented EHW module that acts as a hardware accelerator for the search of this configuration, and uses a small image $(128 \times 128 \mathrm{px})$ for the training in order to reduce the evolution time. This scheme is shown in Figure 7-16.

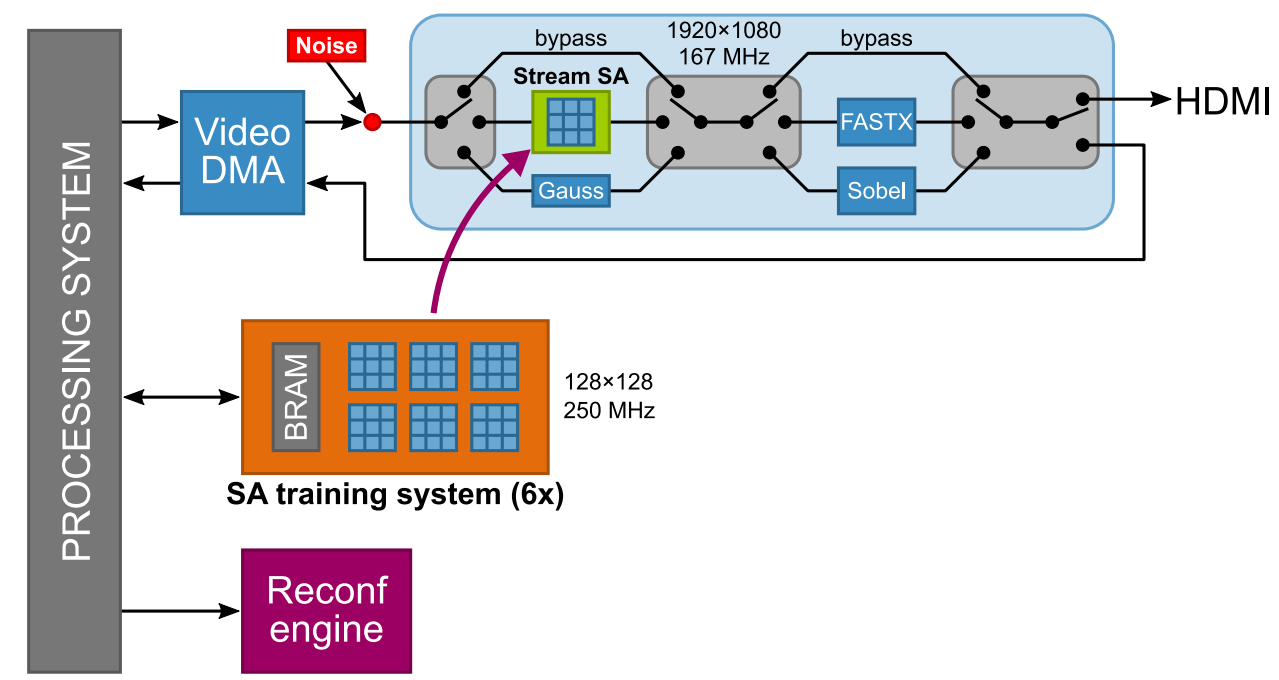

Figure 7-16: Block diagram of the dataflow demonstration application

\footnotetext{
${ }^{20}$ Although these filters could likely be implemented as configurable SA-based filters as well, this has been considered out of the scope of this example.
} 
The system was implemented on a Zedboard development board, which uses a Zynq-7020 all-programmable SoC.

\subsubsection{Description of the different video blocks}

All modules used in this example application have either been developed in previous sections of this thesis or provided by Xilinx (directly or indirectly).

\section{Video processing blocks}

The system uses four video processing blocks: an $8 \times 8$ SA-based reconfigurable image filter, a Gaussian blurring filter (used as an alternative to the SA-based filter for comparison purposes), a Sobel-based edge detection filter, and a corner detection filter based on the FAST algorithm [Rosten'06].

With the exception of the SA-based filter, all blocks have been implemented using the Vivado high-level synthesis (HLS) development tool. This environment provides a high-level C++-based interface for the development of hardware modules, included a large video processing function library based on OpenCV [OpenCV]. Specifically, the functions hls: : GaussianBlur, hls::Sobel, and hls::FASTX [Neuendorffer'15] provide the functionality required for these modules.

All these modules use an AXI4-Stream Video interface for input and output, and are designed to filter frames of sizes up to $1920 \times 1080 \mathrm{px}$.

\section{Noise generation}

In order to demonstrate the video filtering capabilities of the system, a configurable pseudorandom noise generator is added at the video input. This block is based on the one used in the application described in $\S 3.4 .5$.

\section{Video input and output}

Video input is obtained from a static frame stored in SDRAM, streamed using an AXI Video Direct Memory Access (VDMA) block provided by Xilinx. This static frame could be replaced in the future by a sequence of frames or an actual video input from a camera (the latter has not been done due to the lack of a video input on the development board in use). 
Video output can be written back to SDRAM using the write port in the VDMA module, or transmitted to the board's ADV7511 HDMI controller IC in order to be sent to a monitor through the HDMI port.

\section{Interconnection mechanism}

All stream video processing blocks are interconnected either directly or through instances of the AXI4-Stream Switch module from the AXI4-Stream Infrastructure IP Suite [Xilinx-PG085]. This allows routing the video data streams at run time, thus permitting the choice of different video processing blocks.

\section{Evolvable subsystem}

In order to generate the optimal configuration for the SA-based streamoriented reconfigurable image filter, a BRAM-oriented evolvable filtering module with 6 SAs in parallel has been used. This module acts as a hardware accelerator that searches for better solutions while the stream-based one operates with the best solution that has been found so far, thus allowing it to operate at full capacity. This way, the evolution does not interfere with the operation of the system.

This module targets images with a size of $128 \times 128 \mathrm{px}$, and therefore does not use an excessive amount of BRAM.

\subsubsection{Analysis of the resulting system and conclusions}

The resulting system uses a clock frequency of $250 \mathrm{MHz}$ - the highest directly available in Vivado-for the evolvable subsystem and reconfiguration engine, in order to accelerate this part as much as possible. The video processing subsystem uses a clock frequency of $167 \mathrm{MHz}$, allowing it to process a highdefinition $1080 \mathrm{p}(1920 \times 1080 \mathrm{px})$ video stream at frame rates of more than 60 frames per second. Therefore, this system is well suited for real-time video processing applications.

The resource usage of the different modules of this system is shown in Table 7-5. As it can be seen, the stream SA filter is not too big compared with the rest of the filters, and the resource overhead introduced by the reconfiguration engine is quite small. On the other hand, the BRAM-based evolvable system is rather big; however, this system could be made smaller 
by using fewer SAs (at the cost of evolution speed); furthermore, it could be removed using DPR once the evolution has finished, and replaced with other modules.

\begin{tabular}{lrrrr} 
Module & LUT & FF & BRAM & DSP \\
\hline Stream SA filter & 2155 & 1417 & 1 & - \\
Gaussian filter & 697 & 452 & 1.5 & 4 \\
Sobel filter & 1327 & 1189 & 3 & - \\
FAST filter & 3319 & 2225 & 4 & - \\
Switches & 533 & 1103 & - & - \\
\hline BRAM SA system & 13581 & 9874 & 12 & - \\
Reconfiguration engine & 590 & 476 & 1 & - \\
\hline Available in Zynq 7020 & 53200 & 106400 & 140 & 220
\end{tabular}

Table 7-5: Resource usage of the video filtering system

Figure 7-17 shows the result of applying a Sobel filter to an image with $20 \%$ salt and pepper noise without filtering, with a Gaussian blur, and with an SAbased filter respectively. As it can be seen, the results obtained with the evolved filter are considerably better.

This example application shows that, with very little effort, a system that combines a video processing block developed under the proposed design methodology with other preexisting video processing modules can be elaborated. This demonstrates the capabilities of this design methodology, and gives a rough idea of the potential projects that could be developed with it. 

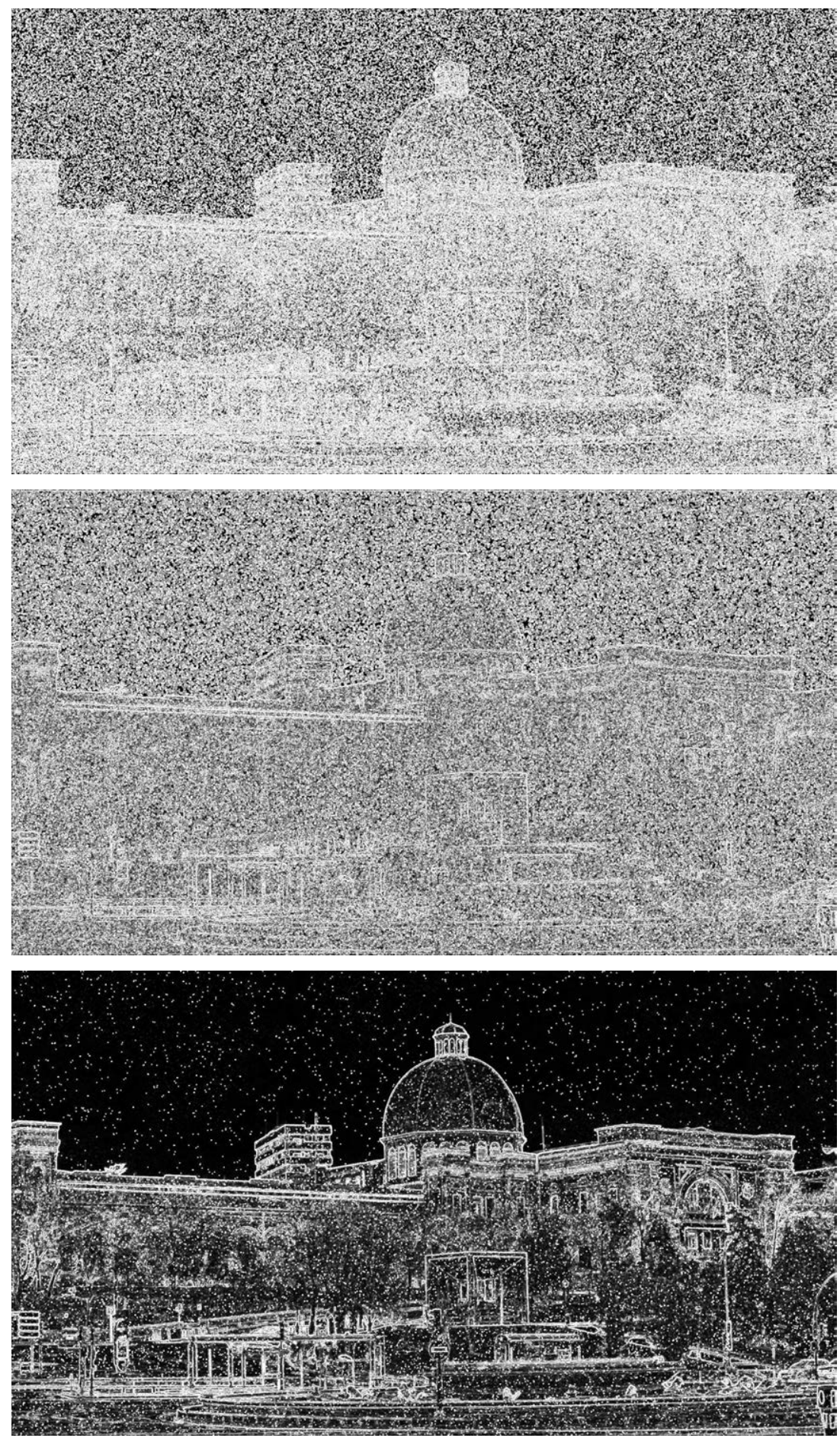

Figure 7-17: Result of applying a Sobel filter to a noisy input (top) and to the same input preprocessed with Gaussian (middle) and evolvable (bottom) filters. 


\section{CONCLUSIONS AND FUTURE LINES}

This chapter concludes the thesis by summarizing its results and establishing a series of future research lines.

First, a summary of the results and conclusions obtained from this thesis is presented in section 8.1. Then, section 8.2 summarizes the main contributions of the thesis to the field of dynamically reconfigurable EHW systems, indicating the sections in which these contributions are elaborated. After that, section 8.3 lists the results of this thesis in terms of publications, related projects, and co-supervised academic works. Finally, section 8.4 enumerates the proposed future work that may be performed in order to perfect and take advantage of the methodology and architecture developed in this thesis.

\subsection{CONCLUSIONS OF THE THESIS}

The first FPGA-based implementations of EHW relied on the direct manipulation of the configuration bitstream. With the emergence of circuit overlays, these implementations became more powerful and easier to implement. This thesis has studied and exploited the use of overlays for the elaboration of EHW systems, analyzing different aspects of their design and implementation in order to tackle the difficulty of their realization.

This work has revolved around a specific application of an EHW systeman evolvable image filter based on a reconfigurable systolic array-, which has served as a proof of concept for demonstrating the theoretical and practical contributions of this thesis. However, it has been shown how these contributions are not limited to this specific application, providing examples of the generalizability of these results and developing a design methodology that is agnostic to the application and focuses on the usefulness of these results in future projects. 
The design methodology elaborated in this thesis follows the principles of performance, scalability, and generalizability, aiming at simplifying the implementation of EHW systems which are able to evolve in a short time and that have a high performance once implemented. This has been tackled by defining a fast and versatile reconfiguration technique as well as an optimized processing architecture, which ensure the quick adaptation of the EHW system to specific problems. The benefits contributed by this methodology are reflected in a considerable acceleration of the proof-of-concept EHW system, resulting in an evolution speed of 187000 candidate solution evaluations per second - which, to the best of the author's knowledge, is the highest evaluation speed ever reported - and a reduction of the total evolution time to one second for many applications. The resource efficiency of the design methodology has allowed the implementation of processing architectures of a high compacity, leading to implementations of evolvable processing arrays of up to 576 PEs that can operate at speeds of up to $500 \mathbf{~ M H z}$ on the FPGA at hand, being able to process image data at a rate of 500 million grayscale pixels per second, equivalent to $4 \mathrm{~K}$ UHD $(3480 \times 2160)$ at 60 frames per second.

The elaboration of this thesis has involved a series of exhaustive experimental tests in which approximately 30 billion candidate configurations were evaluated in total, performing an estimate of 200 billion partial reconfigurations. ${ }^{1}$ This shows the importance of a fast and reliable reconfiguration and evaluation mechanism, which not only benefits the implementation of EHW systems but also their experimental validation and analysis.

In addition to the design methodology, several hardware modules and software tools have been developed, aiming at the creation of systems that integrate EHW in a future. With this, the author wants to demonstrate that the work of this thesis has practical applications that may be helpful for future research and development in the field of EHW.

\footnotetext{
${ }^{1}$ This only considers the experiments whose results are described in this document, and not any discarded experiments or demonstrations.
} 


\subsection{SUMMARY OF MAIN CONTRIBUTIONS}

In this thesis, a series of design techniques that facilitate the implementation of EHW systems based on DPR have been defined. These techniques have been validated through the implementation of an EHW system-a sliding window image filter based on a reconfigurable SA-and its experimental assessment under different working conditions, studying its behavior and comparing the results of different design variations. Although the development of these techniques has revolved around a particular implementation of EHW, they have been conceived with generalizability in mind, aiming at their usability in future projects.

In addition, several secondary contributions in the form of ready-to-use hardware modules and software tools have been made. These contributions serve as a demonstration of the methodology, and may be useful for future projects where the low resource usage and high flexibility and adaptivity of DPR-based EHW may be beneficial.

This section summarizes all these contributions-methodological, analytical, and in the form of developed utilities. The impact of these contributions is reflected as progressive improvements on the EHW system at hand in terms of resource usage, scalability, and performance; a series of tables summarizing the evolution of those along the thesis is provided at the end of the section.

\subsubsection{Methodological contributions}

This thesis has contributed the following methods and techniques to the field of DPR-based EHW:

- A design methodology for the implementation of dynamically reconfigurable hardware on Xilinx FPGAs. This methodology relies on the use of LUT-based DPR ( $\$ 5.1)$ to be performed with a hardware-accelerated finegrain DPR engine $(\$ 4.1)$, as well as the development of highly reusable modules for the fast evaluation of candidate solutions (\$ 5.3) and for the implementation of dataflow-based video processing systems (\$ 5.4).

This methodology is characterized by:

- A high degree of parameterization and modularity, aiming at reusability of the design and ease of maintenance and future extension. 
- Automatic generation of constraints necessary for component placement and placement prohibition within the HDL code, removing the need of additional work other than component instantiation once the core has been designed.

- High performance of the design in terms of speed, resource usage, and quality of results.

- Scalability, both in the size of the processing modules and the number of them.

- A minimization of the software overhead necessary to control the resulting cores.

- A simplified design: unnecessary complexity not only hinders the maintenance and extension of the developed cores but may also reduce the maximum operating frequency of the resulting hardware.

This methodology seeks two goals: to make the developed hardware modules easy to adopt/instantiate/use once developed, and to make them as versatile and flexible as possible.

- A methodology for the analysis of potentially beneficial PE functions and the generation of new PE function libraries $(\$ 7.2)$. This methodology has allowed elaborating an improved PE function library that reduces the fitness value for the salt and pepper noise problem by $14 \%$, while performing satisfactorily for other problems such as shade removal or Sobel filter imitation.

- A series of methods for the analysis and comparison of the quality of the developed hardware $(\$ 5.2)$ and algorithms $(\$ 6.1)$. These methods have been useful for defining the optimal parameters of the architecture and EA, as well as the assessment of the impact of possible future modifications.

- A selection of strategies to accelerate the evolutionary process of the developed EHW system. These strategies consist in:

- Improving the EA in use $(\$ 6.1)$ and providing a methodology for adjusting its parameters for a better performance under different use cases. This improved EA reaches similar results as before in one fifth as many candidate solution evaluations, resulting in an acceleration of the evolution time of 5.5 times. 
- Exploiting the parallelizability of the developed system ( $\$ 6.2)$, providing a methodology to analyze the optimal strategy and parallelism level based on the filtering, reconfiguration, and software times. This parallelization further reduces the evolution time by 6.8 times.

The combination of these two strategies has provided an overall reduction of the evolution time from 51.6 to 1.38 seconds, i.e., more than 37 times faster.

\subsubsection{Analytical contributions}

The following are contributions of this thesis in the form of extensive experimental analyses:

- A comparative analysis between two candidate topologies for the implementation of dynamically reconfigurable EHW in FPGAs-SA and CGP-, the study of their scalability, and a proposal for the improvement of the SA topology at hand ( $(5.2)$. This analysis has shown that an SA with a size of $10 \times 10$ PEs outperforms an equivalent CGP implementation of $5 \times 10$ PEs while using half as many resources, making it the preferable topology for this kind of system. Additionally, it shows that the SA topology scales satisfactorily for sizes up to $10 \times 10$ but shows no improvement above sizes of $12 \times 12$. CGP exhibits a similar behavior: it scales smoothly for sizes up to $5 \times 10$, but its improvement with size slows down past this point, reaching a limit at $10 \times 20$.

- A brief study of the performance of the proposed system under different problems and the individual contributions of each PE function $(\S 7.2$ ). This study reveals that some functions (such as the constant value of 255) do not provide a great benefit in general, whereas some new functions (such as the half-difference) may be highly beneficial for certain problems such as shade removal. 


\subsubsection{Developed hardware modules}

In addition to theoretical and methodological research results, this thesis has produced practical contributions in the form of hardware and software assets. The hardware modules and systems developed in this thesis, which may be beneficial in future work (either by direct use or for further development), are:

- A fully parameterized LUT-based systolic array module for Virtex-5 and 7 Series FPGAs ( $\$ 5.1)$. This module is primarily intended for processing image data, but may be used for any other type of data. This module is targeted towards reusability. The module has been designed so that all necessary constraints are integrated in its HDL code, simplifying its use (this is part of the design methodology). The parameters that can be configured are:

- PE sample data width.

- SA height and width.

- Number of inputs and delay levels per input (which correspond to height and width of a sliding window).

- Single- or dual-output PEs (as used in $\$ 5.2 .4$ for implementing bypassable PEs).

- SA position within the FPGA.

This SA is scalable at design time and highly compact, using $100 \%$ of LUTs locally available in the FPGA area it covers and a very small number of LUTs per PE. This compactness results in short interconnections and thus high operating frequencies, which go up to $500 \mathrm{MHz}$ for sizes of $8 \times 8$ PEs, and up to $450 \mathrm{MHz}$ for $24 \times 24$ (for a Virtex-5 FPGA). Additionally, the array has been designed so that its functionality can be changed by reconfiguring a reduced number of frames (affecting only its LUTs), thus minimizing the reconfiguration time.

- A highly parameterized SA-based image processing and fitness computing peripheral with parallel evaluation capabilities for Virtex-5 and 7 Series FPGAs $(\$ 5.3)$. This peripheral can be used either for filtering small images with a fixed SA configuration or for evaluating the fitness of several SA configurations in parallel (as a hardware accelerator for the fitness computation of an EA). The filter is based on a sliding window of parameterizable size that feeds pixels to an SA. The following parameters can be configured in addition to the ones from the SA itself: 
- Number of SAs.

- Height and width of the image.

- Clock speed of the different parts (SA, memory access, fitness computation).

- Parallelization level of fitness computing units (useful for reducing their clock speed).

- An SA-based streaming video processing core with AXI4-Stream Video interface for 7 Series FPGAs (\$5.4). This interface allows for an easy integration with preexisting video processing cores, thus promoting the use of this core for constructing more complex processing systems. This core can process arbitrarily large video frame sizes, which can be changed at run time; specifically, it is able to process high-definition video $(1920 \times 1080 \mathrm{px}$ frames at 60 frames per second).

- A fast and versatile ICAP-based fine grain reconfiguration engine for Virtex-5 and 7 Series FPGAs, supporting sub-frame reconfiguration via on-the-fly composition of multiple PBSs (\$4.1), with which a highly parameterized reconfiguration peripheral has been built ( $\$ 7.2 .2)$. The engine can be customized for arbitrary sub-frame layouts and multiple use cases, so it is reusable in other projects, not being limited to SA nor LUT-based reconfiguration. This reconfiguration engine has been tested for speeds of up to $300 \mathrm{MHz}$.

- Several applications integrating these modules and demonstrating their capabilities:

- A demonstration application showing the evolution and real-time operation of the system $(\$ 3.4 .5)$.

- A system based on a very large SA $(24 \times 24$ PEs $)$ used to study its scalability (\$5.2).

- An application demonstrating the ability of the system to adapt to unknown noise types without using a noise-free training reference $(\S 7.1)$.

- An evaluation platform to study the performance of the system with different sliding window sizes and PE function libraries under different problems (\$ 7.2).

- An example video filtering application demonstrating the operation of the stream-based filter and its integration with other video processing components $(\$ 7.3)$. 
These applications provide a means of dissemination of research results in the form of conference demos and publications.

\subsubsection{Developed software tools}

Finally, this work has yielded a series of software tools and applications which may be useful in future projects:

- A simulation platform for both SA $(\$ 3.4 .1)$ and CGP $(\$ 5.2 .1)$ image filters with a functionally identical behavior to the hardware counterpart. This platform allows analyzing and comparing the behavior of SA and CGP and different potential modifications without needing to implement them in hardware. Additionally, it serves as a tool for checking that a hardware implementation behaves as expected.

- A partial bitstream extraction tool that can be easily extended to support additional FPGAs ( $\$ 4.2$ ). This tool has been used to obtain the PBS of PEs in early stages of this project, and has also been useful in other projects related to DPR.

- A script to automate the generation of exact or approximate PEs within the constraints imposed by the PE model described in $\$ 5.1 .2$ (\$7.2.4). This script may be useful for implementing new arbitrary functions for the proposed SA architecture.

\subsubsection{Summary of improvements on the hardware platform}

To conclude this section, a summary of the incremental improvements to the original hardware platform described in chapter 3 in terms of resource usage, scalability, and acceleration of the evolution time is provided. These improvements reflect the practical implications of the contributions of this thesis.

Table 8-1 shows the improvement in resource usage per PE, and how it has impacted in the available array size and number of arrays implemented on the system.

Table 8-2 summarizes the improvement on the evolution time, both as a reduction of the evaluation time (as a combination of reconfiguration, filtering, and software times) and as a reduction in the number of evaluations, and shows the incremental acceleration of the evolution stage caused by this improvement. As it can be seen, this stage has been accelerated near 500 times with respect to the initial system described in chapter 3. 


\begin{tabular}{|c|c|c|c|c|}
\hline \multicolumn{2}{|c|}{ Section } & PE size & Array size & Arrays \\
\hline \multicolumn{5}{|c|}{ Initial system } \\
\hline$\$ 3.1$ & Full frame reconfiguration & 40 CLBs & $4 \times 4$ & 1 \\
\hline$\S 3.1 .2$ & Sub-frame reconfiguration & 10 CLBs & $4 \times 4$ & 1 \\
\hline$\S 3.1 .2$ & DREAMS tool routing & 5 CLBs & $8 \times 7$ & 1 \\
\hline \multicolumn{5}{|c|}{ Thesis contributions } \\
\hline$\S 5.1$ & LUT-based DPR & 2 CLBs & $8 \times 8$ & 1 \\
\hline$\S 5.3 .4$ & Parameterized system & 2 CLBs & $8 \times 8$ & 16 \\
\hline
\end{tabular}

Table 8-1: Progression of the resource usage and scalability

\begin{tabular}{|c|c|c|c|c|c|c|c|}
\hline \multirow{2}{*}{\multicolumn{2}{|c|}{ Section }} & \multicolumn{3}{|c|}{ Time $(\mu s)$} & \multirow[t]{2}{*}{ Evals. } & \multirow[t]{2}{*}{ Total (s) } & \multirow[t]{2}{*}{ Accel. } \\
\hline & & Rcfg & Filt & SW & & & \\
\hline \multicolumn{2}{|c|}{ Initial system $^{2}$} & 333 & 166 & 2.5 & $960 \mathrm{k}$ & 481 & \\
\hline \multicolumn{8}{|c|}{ Hardware improvements } \\
\hline$\S 4.1$ & DPR engine & 74 & 166 & 2.5 & $960 \mathrm{k}$ & 233 & $\times 2.1$ \\
\hline$\S 5.1$ & LUT-based SA & 9.3 & 42 & 2.5 & $960 \mathrm{k}$ & 51.6 & $\times 4.5$ \\
\hline \multicolumn{2}{|l|}{ Total } & & & & & & $\times 9.3$ \\
\hline \multicolumn{8}{|c|}{ EA improvements } \\
\hline$\S 6.1 .2$ & Mutation rate & 7.5 & 42 & 1.8 & $960 \mathrm{k}$ & 49.2 & $\times 1.05$ \\
\hline$\S 6.1 .3$ & Offspring size & 7.4 & 42 & 1.8 & $960 \mathrm{k}$ & 49.1 & $\times 1$ \\
\hline$\S 6.1 .4$ & Best of 12 & 7.4 & 42 & 1.8 & 480k & 24.6 & $\times 2$ \\
\hline$\S 6.1 .5$ & Exchanges & 7.4 & 42 & 1.8 & 192k & 9.83 & $\times 2.5$ \\
\hline$\S 6.1 .6$ & Single column & 5.2 & 42 & 1.8 & $192 \mathrm{k}$ & 9.41 & $\times 1.05$ \\
\hline \multicolumn{2}{|l|}{ Total } & & & & & & $\times 5.5$ \\
\hline \multicolumn{8}{|c|}{ EA parallelization } \\
\hline$\$ 6.2 .1$ & 12 parallel SAs & 5.2 & 3.5 & 1.8 & $192 \mathrm{k}$ & 2.01 & $\times 4.6$ \\
\hline$\S 6.2 .2$ & Half-and-half & 5.2 & $(\mathbf{0})$ & 2.0 & $192 k$ & 1.38 & $\times 1.5$ \\
\hline \multicolumn{2}{|c|}{ Total } & & & & & & $\times 6.8$ \\
\hline \multicolumn{8}{|c|}{ Further improvements } \\
\hline$\S 7.2 .2$ & Generalized engine & 3.7 & (0) & 1.7 & $192 \mathrm{k}$ & 1.03 & $\times 1.3$ \\
\hline \multicolumn{2}{|l|}{ Total } & & & & & & $\times 1.3$ \\
\hline \multicolumn{2}{|c|}{ Overall total } & & & & & & $\times 467$ \\
\hline
\end{tabular}

Table 8-2: Incremental improvements on the evolution time

\footnotetext{
${ }^{2}$ Using 10-CLB PEs.
} 


\subsection{IMPACT OF THE THESIS}

This section summarizes the academic results of this thesis in the form of publications, related projects, and co-supervised works.

\subsubsection{Publications and dissemination}

During this thesis, multiple articles and papers have been published showing the research proposals and experimental results. In addition, scientific dissemination has also been achieved in the form of conference demonstrations (most of them using the demo application developed in $\$ 3.4 .5$ and its ongoing improvement as a result of this thesis).

\section{Journal publications}

The author of this thesis has coauthored three journal articles published in Q2Q3 JCR journals:

[Mora'18a] J. Mora, R. Salvador, E. de la Torre, “On the scalability of evolvable hardware architectures: comparison of systolic array and Cartesian genetic programming", in Genetic Programming and Evolvable Machines, Oct. 2018, ISSN 1573-7632, doi:10.1007/s10710-018-9340-5. 2017 JCR impact factor: $1.455(\mathrm{Q} 2)$.

This article presents the comparative analysis between CGP and SA in terms of scalability and resource usage described in $₫ 5.2$.

[Mora'18b] J. Mora, E. de la Torre, "Accelerating the evolution of a systolic array-based evolvable hardware system", in Microprocessors and Microsystems, vol. 56, pp. 144-156, Feb. 2018, ISSN 0141-9331, doi: 10.1016/j.micpro.2017.12.001. 2017 JCR impact factor: 1.049 (Q3).

This article summarizes the efforts targeting the acceleration of the evolution, including both the hardware improvements made in $\$ 4.1$, $\S 5.1$, and $\$ 5.3$, the EA improvement from $\$ 6.1$, and the parallelization strategy described in $\$ 6.2$.

[Salvador'13] R. Salvador, A. Otero, J. Mora, E. de la Torre, T. Riesgo, L. Sekanina, "Self-Reconfigurable Evolvable Hardware System for Adaptive Image 
Processing", in IEEE Transactions on Computers, vol. 62, no. 8, pp. 14811493, Aug 2013, ISSN 0018-9340, doi:10.1109/TC.2013.78. 2013 JCR impact factor: $1.473(\mathrm{Q} 2)$.

This article describes the generalizability and adaptivity properties of the EHW system described in $§ 3.3$.

\section{Conference publications}

The following are all coauthored conference publications published during the time span of this thesis:

[Zamacola'18] R. Zamacola, A. García, J. Mora, A. Otero, E. de la Torre, "IMPRESS: Automated Tool for the Implementation of Highly Flexible Partial Reconfigurable Systems with Xilinx Vivado”, in 2018 International Conference on Reconfigurable Computing and FPGAs (ReConFig), Dec. 2018, ISSN 2325-6532.

This paper presents a tool for the generation of relocatable PBSs in Vivado, permitting a similar design methodology to the one provided by the DREAMS tool in ISE.

[Mora'15] J. Mora, A. Otero, E. de la Torre, T. Riesgo, "Fast and compact evolvable systolic arrays on dynamically reconfigurable FPGAs", in Reconfigurable Communication-centric Systems-on-Chip (ReCoSoC), 2015 10th International Symposium on, pp. 1-7, June 2015, doi: 10.1109/ReCoSoC.2015.7238087.

This paper introduces the compact LUT-based SA implementation described in $\$ 5.1$ and its parallelization capabilities.

[Gallego'13c] Á. Gallego, J. Mora, A. Otero, E. de la Torre, T. Riesgo, "A scalable evolvable hardware processing array", in 2013 International Conference on Reconfigurable Computing and FPGAs (ReConFig), pp. 1-7, Dec 2013, ISSN 2325-6532, doi:10.1109/ReConFig.2013.6732266.

This paper presents a dynamically scalable SA-based EHW system, motivating the scalability parameterization and analysis carried out in $\S 5.1$ and $\S 5.2$.

[Veljković'13] F. Veljković, J. Mora, T. Riesgo, L. Berrojo, R. Regada, Á. Álvaro, E. de la Torre, "Prospection of Reconfiguration Capabilities using Space 
Qualified SRAM-based FPGAs for a Satellite Communications Application", in 31st AIAA International Communications Satellite Systems Conference, International Communications Satellite Systems Conferences (ICSSC), American Institute of Aeronautics and Astronautics, Oct. 2013, doi:10.2514/6.2013-5683.

This paper demonstrates the benefits of DPR in terms of power consumption and resource usage for a space application.

[Mora'13b] J. Mora, Á. Gallego, A. Otero, E. de la Torre, T. Riesgo, "Noiseagnostic adaptive image filtering without training references on an evolvable hardware platform", in Design and Architectures for Signal and Image Processing (DASIP), 2013 Conference on, pp. 182-189, Oct 2013, http://ieeexplore.ieee.org/document/6661538/.

This paper proposes a strategy for obtaining a training data set from a noisy input for use in real-time video processing systems where the type of noise may not be known, as described in $\$ 7.1$.

[Gallego' 13b] Á. Gallego, J. Mora, A. Otero, R. Salvador, E. de la Torre, T. Riesgo, "A Novel FPGA-based Evolvable Hardware System Based on Multiple Processing Arrays", in 2013 IEEE International Symposium on Parallel Distributed Processing, Workshops and Phd Forum, pp. 182-191, May 2013, doi:10.1109/IPDPSW.2013.56.

This paper proposes several use cases for a triple SA-based EHW system, both for higher computational capabilities and for fault tolerance. The necessity of designing this type of cascaded EHW systems motivated the development of the dataflow-based design approach proposed in $\$ 5.4$.

\section{Previous conference publications}

The following are publications under the scope of the research covered by this thesis but that were published prior to its start.

[Salvador'12] R. Salvador, A. Otero, J. Mora, E. de la Torre, T. Riesgo, L. Sekanina, "Implementation techniques for evolvable HW systems: virtual VS. dynamic reconfiguration”, in 22nd International Conference on Field Programmable Logic and Applications (FPL), pp. 547-550, Aug. 2012, ISSN 1946-147X, doi:10.1109/FPL.2012.6339376.

[Salvador'11b] R. Salvador, A. Otero, J. Mora, E. de la Torre, L. Sekanina, T. Riesgo, "Fault Tolerance Analysis and Self-Healing Strategy 
of Autonomous, Evolvable Hardware Systems", in 2011 International Conference on Reconfigurable Computing and FPGAs, pp. 164-169, Nov 2011, ISSN 2325-6532, doi:10.1109/ReConFig.2011.37.

[Otero'11a] A. Otero, R. Salvador, J. Mora, E. de la Torre, T. Riesgo, L. Sekanina, "2D Reconfigurable Systolic Core Architecture for Evolvable Systems", in Proceedings of the XXVI Conference on Design of Circuits and Integrated Systems (DCIS 2011), Nov. 2011.

[Salvador'11a] R. Salvador, A. Otero, J. Mora, E. de la Torre, T. Riesgo, L. Sekanina, "Evolvable 2D computing matrix model for intrinsic evolution in commercial FPGAs with native reconfiguration support", in Adaptive Hardware and Systems (AHS), 2011 NASA/ESA Conference on, pp. 184-191, June 2011, doi:10.1109/AHS.2011.5963934.

[Otero'11b] A. Otero, R. Salvador, J. Mora, E. de la Torre, T. Riesgo, L. Sekanina, "A fast Reconfigurable 2D HW core architecture on FPGAs for evolvable Self-Adaptive Systems", in Adaptive Hardware and Systems (AHS), 2011 NASA/ESA Conference on, pp. 336-343, June 2011, doi: 10.1109/AHS.2011.5963956.

\section{Other dissemination channels}

In addition to conference papers and journal articles, further dissemination in the form of demos, posters, and oral presentations were performed.

[López'14] B. López, J. Mora, P. Mansanet, E. de la Torre, T. Riesgo, "Development of Brain-Computer Interfaces using Evolvable Hardware", in 2014 Conference on Design and Architectures for Signal and Image Processing (DASIP), pp. 1-1, IEEE, 2014, http://oa.upm.es/37019/. Poster presentation.

[Mora'14] J. Mora, Á. Gallego, A. Otero, E. de la Torre, T. Riesgo, "Increased fault tolerance in evolvable hardware through automatic upscaling", in Conference on Design and Architectures for Signal and Image Processing (DASIP 2014), pp. 1-1, 2014, http://oa.upm.es/37142/. Demo.

[Valverde'14] J. Valverde, A. Rodríguez, J. Mora, C. Castañares, J. Portilla, E. de la Torre, T. Riesgo, "A Dynamically Adaptable Image Processing Application Trading Off Between High Performance, Consumption and Dependability in Real Time", 2014, http://oa.upm.es/37015/. Demo. 
[Mora'13c] J. Mora, Á. Gallego, A. Otero, E. de la Torre, T. Riesgo, "A noiseagnostic self-adaptive image processing application based on evolvable hardware", in 2013 Conference on Design and Architectures for Signal and Image Processing, pp. 351-352, Oct. 2013, https://ieeexplore.ieee. org/document/6661571/. Demo.

[Gallego'13a] Á. Gallego, J. Mora, A. Otero, B. López, E. de la Torre, T. Riesgo, "A self-adaptive image processing application based on evolvable and scalable hardware", in 2013 23rd International Conference on Field programmable Logic and Applications (FPL), pp. 1-1, Sep. 2013, ISSN 1946-147X, doi:10.1109/FPL.2013.6645631. Demo.

[Otero'12b] A. Otero, J. Mora, R. Salvador, Á. Gallego, E. de la Torre, L. Sekanina, "Evolvable hardware FPGA-based platform for autonomous faulttolerant systems", in 2012 International Conference on ReConFigurable Computing and FPGAs (ReConFig), Demo Night, Cancun (Mexico), Dec. 2012. Demo.

[Otero'12a] A. Otero, J. Mora, R. Salvador, Á. Gallego, E. de la Torre, L. Sekanina, "Evolvable hardware FPGA-based platform for autonomous fault-tolerant systems", in 2012 Design, Automation \& Test in Europe (DATE), University Booth, Dresden, Germany, Mar. 2012. Demo.

[Mora'12] J. Mora, "Self-healing evolvable hardware architectures for image processing applications", Mar. 2012, http://www.cei.upm.es/media/ seminarios/2012/Presentations/4_4_Seminario_CEI_2012_SelfHealing.pdf. Oral presentation.

\subsubsection{Related projects and research lines}

This thesis has been elaborated under the scope of projects DREAMS (TEC2011-28666-C04) and REBECCA (TEC2014-58036-C4-2-R) from the Spanish Ministry of Economy and Competitiveness. These projects target the exploration of dynamically reconfigurable systems and their application to multimedia systems and smart cities.

Additionally, the author has collaborated in the European project FASTCUDA (FP7-SME-2011 286770), which targeted the development of hardwaresoftware codesign tools for the implementation of CUDA kernels on reconfigurable platforms.

Regarding academic research, although this thesis has primarily focused on the study of SA-based evolvable image filters, the author has explored 
and been involved in parallel research lines related to DPR and EHW. One of these research lines has focused on the possibility of implementing a low-level routing tool similar to DREAMS using Vivado's Tcl scripting, which would allow the automated generation of routing constraints for DPR modules relying on custom HDL attributes. However, the adoption of the LUT-based approach has made these routing constraints unnecessary for this thesis, making this research line less prioritary. Nevertheless, this research line has remained active and is being used in current projects [Zamacola'18].

Another complementary research line the author has been involved in is the development of systems for the acquisition, processing, and classification of electroencephalographic (EEG) signals targeting the development of braincomputer interfaces (BCIs). This line of research had the goal of finding possible applications of SA-based evolvable signal processing systems other than image filtering, and showed some of the potential requirements and necessary improvements of the system at hand such as the need for higher precision PEs and larger processing arrays. This research line has shown the feasibility of using SA-based EHW systems from a theoretical point of view [López'14], leaving the implementation of matching hardware systems for future work.

Finally, there is an ongoing collaboration with a parallel research line focused on the $A R T I C o^{3}$ hardware architecture [Rodríguez'18]. This research line aims at developing hardware-software systems on all-programmable SoCs through the use of dynamically reconfigurable hardware accelerators, and thus shares many common goals with this thesis. Therefore, there is an ongoing work of integration of some of the products of this thesis, such as the partial bitstream extraction tool developed in $\$ 4.2$ or the SA-based image processing filter. $^{3}$

\subsubsection{Co-supervised works}

This thesis has represented an opportunity for academic research, leading to the co-direction of multiple B.Sc. theses and proyectos fin de carrera (B.Sc./M.Sc. final projects) ${ }^{4}$ presented in the Universidad Politécnica de

\footnotetext{
${ }^{3}$ Specifically, there is an ongoing integration of the SA filter as an ARTICo ${ }^{3}$ reconfigurable accelerator, which would make use of DPR at two levels: coarse grain (reconfiguration of a whole accelerator) and fine grain (reconfiguration of individual PEs).

${ }^{4}$ In Spain, the proyecto fin de carrera was the final project elaborated at the end of the long-cycle engineering degree (equivalent to a bachelor's and a master's degree) prior to international normalization through the Bologna Process.
} 
Madrid. These theses not only served academic and formative purposes, but also provided valuable results for use within this research line.

[González'17] C. González, Filtrado mediante hardware evolutivo de imágenes de tamaño arbitrario sobre plataforma Zynq, B.Sc. thesis, Universidad Politécnica de Madrid, 2017, http://oa.upm.es/45517/.

This B.Sc. thesis explored the possibility of using a window generator for variable width images as a means to filter real-time video of arbitrary size. This development eventually led to the stream-based video filter implementation described in $\$ 5.4$.

[Barajas'17] R. Barajas, Análisis y optimización de algoritmos genéticos para el filtrado de imágenes mediante hardware evolutivo, B.Sc. thesis, Universidad Politécnica de Madrid, Feb. 2017, http://oa.upm.es/45666/.

In this B.Sc. thesis, several parameters of the EA such as the mutation rate and possible crossover mechanisms are analyzed, along with their effect on arrays of different sizes. These results were important for the elaboration of the experimental assessment methodology used in $\$ 5.2$, and pose some ideas for future research in the topic of the EA.

[Correa'17] C. J. Correa, Adquisición y procesamiento multisensorial en FPGA para EEG, B.Sc. thesis, Universidad Politécnica de Madrid, Feb. 2017, http://oa.upm.es/45521/.

This B.Sc. thesis developed a physical system for EEG signal acquisition, as part of the research line focused on BCI.

[Iglesias'16] P. Iglesias, Brain-Computer Interfaces: Desarrollo de un sistema de identificación de estados mentales alfa, B.Sc. thesis, Universidad Politécnica de Madrid, Feb. 2016, http://oa.upm.es/39564/.

This B.Sc. thesis demonstrated a hardware implementation of an EEG signal identification.

[Conejo'15] R. Conejo, Brain-computer interfaces: desarrollo de sistema de adquisición de datos, B.Sc. thesis, Universidad Politécnica de Madrid, Jul. 2015.

This B.Sc. thesis implemented a hardware interface for the EEG signal acquisition system.

[Rojas'15] P. Sánchez de Rojas, Ejecución de aplicaciones multihilo sobre sistemas en FPGA con aceleradores hardware descritos en CUDA, B.Sc./M.Sc. 
final project, Universidad Politécnica de Madrid, Sep. 2015, http://oa. upm.es/43483/.

This B.Sc./M.Sc. final project focused on the development of (potentially reconfigurable) hardware accelerators described in CUDA for the FASTCUDA project [Mavroidis'12].

[Mansanet'14] P. Mansanet, Biblioteca para modelado y simulación de alto nivel de sistemas evolutivos complejos implementados en hardware, B.Sc./M.Sc. final project, Universidad Politécnica de Madrid, Sep. 2014.

In this B.Sc./M.Sc. final project, a library for modeling and simulating complex SA-based evolvable systems was developed. This thesis provided some insight into the possibilities of dual-output PEs and improvements on the EA.

[Polop'14] F. J. Polop, Implementación de filtros de eliminación de moteado de imágenes mediante hardware evolutivo iterativo, B.Sc. thesis, Universidad Politécnica de Madrid, Sep. 2014.

This B.Sc. thesis explored the use of cascaded SA-based evolvable image filters for the removal of speckle noise.

[Fernández'14] V. Fernández, Modelado de hardware evolutivo sobre plataformas CUDA, B.Sc. thesis, Universidad Politécnica de Madrid, Sep. 2014.

This B.Sc. thesis implemented a GPU-accelerated version of the SA-based system software simulation. In addition to a faster simulation of SA systems, this could be useful in the context of distributed heterogeneous evolvable systems (CPU, GPU, and FPGA).

\subsection{FUTURE WORK}

This thesis has presented a very versatile EHW platform intended for signal processing accompanied by a DPR implementation methodology and tools. However, several aspects of this thesis may be further improved and explored in the future, ranging from experimental analyses of the current platform under different conditions to potential improvements on the architecture and the exploration of possible applications. 


\subsubsection{Experimental analysis and exploitation of the current architecture}

An aspect that may be considered for future research is the study and exploitation of the possibilities provided by the proposed architecture.

In $\$ 5.2 .4$, it was shown how the use of bypassable PEs greatly improves the SA performance. However, this approach has barely been explored in this thesis: it would be worthwhile to perform a more in-depth analysis of this topology variation, studying how to better adapt the EA and DPR methodology to it in order to obtain optimal results. In addition, this two-output model not only allows bypassing the PEs, but also combining two PE functions in one PE as long as they have the same stage 1 (for example, maximum and minimum). Thus, a more complex PE function library with some of these combinations could be specified and studied. Previous work [Mansanet'14] suggests that these dual PEs may greatly improve the performance of the system.

Another interesting line of research would be the overall improvement of the PE function library. The experimental methodology proposed in $§ 7.2 .4$ has the flaw of only analyzing the individual contribution of each PE function, without considering how two functions may interact positively or negatively (for example, redundant functions would interact negatively, whereas functions that may be used together would interact positively); a study that takes into account these interactions (and explores a wider set of problems than the five used in $\$ 7.2 .4$ ) may yield better results than the proposed methodology. In addition, it should be considered whether the size of the PE function library should be extended or reduced. Furthermore, it may be possible to implement an EA that directly chooses which subset of functions it uses at run time. Alternatively, a finer-grain EA could be used that creates new functions on the fly, either by evolving the two stages separately or by evolving LUT content bits individually.

Finally, a feature that has not been explored is the use of different PE data widths, such as 16 or 32 bits. This may be useful for processing extended precision images or for an eventual exploration of other types of signal. Although it is expected that the EA will perform similarly regardless of the data width, this should be experimentally assessed. 


\subsubsection{Evolutionary algorithm}

As it was shown in $\$ 6.1$, a small effort on improving the EA can represent a high speedup in the evolution stage. So far, the current system is able to evolve in one second for the current use case. Nevertheless, it may be possible that this were still too slow for some real-time applications with tight speed requirements. In addition, the fact that the evolution fitness keeps improving during the evolution without apparently reaching a defined limit suggests that the maximum potential of the processing architecture is not being reached. Therefore, further research in the EA may be advisable.

One possibility to explore would be the inclusion of crossover in order to make a proper GA. The EA used in this thesis did not use crossover because previous work [Vassilev'99, Sekanina'04] states that uniform crossover is an inefficient operator for the evolution of CGP (and it was assumed that this would also apply to SA). This is probably due to uniform crossover operating on a one-dimensional genotype, whereas CGP and SA have a two-dimensional layout. Nevertheless, crossover operators intended for two-dimensional architectures exist [Moon'01], and some experiments with a similar crossover mechanism have shown that this strategy may be beneficial [Barajas'17, ch.7]. The introduction of crossover would open multiple possibilities for advanced selection mechanisms (tournament, roulette-wheel...) and extended population sizes. In addition to crossover, it might be worth considering the change to a bit-level mutation rather than gene-level (with one gene being represented by a few bits), as is customary in GA.

Other improvements that could be made on the EA are parametric improvements, such as fine-tuning mutation rate and offspring size (or making them vary as the evolution progresses), and implementing more sophisticated evolutionary techniques that achieve good results with fewer candidate solution evaluations.

\subsubsection{Architecture}

Although the processing architecture has been highly optimized and generalized, there are still some improvements that are worth exploring in the future.

One of the possible points of improvement for the architecture is the use of larger input windows. As it can be seen in Figure 8-1, the size of the sliding window could be increased to up to $6 \times 9$ ( 6 input channels, 9 possible delay values) without needing more reconfigurable LUTs. Outside of image filtering, this may be useful for implementing FIR filters on multi-channel signals; an 
example of such an application would be EEG signal processing. However, experimental tests performed in $\$ 7.2 .3$ show that the current EA is not efficient at using large windows, so it should be improved in this aspect in order to take advantage of the larger windows. One way to achieve this improvement would be to make the EA choose a subset of the input window so that only those pixels are used, as is done in [Vašíček'09].

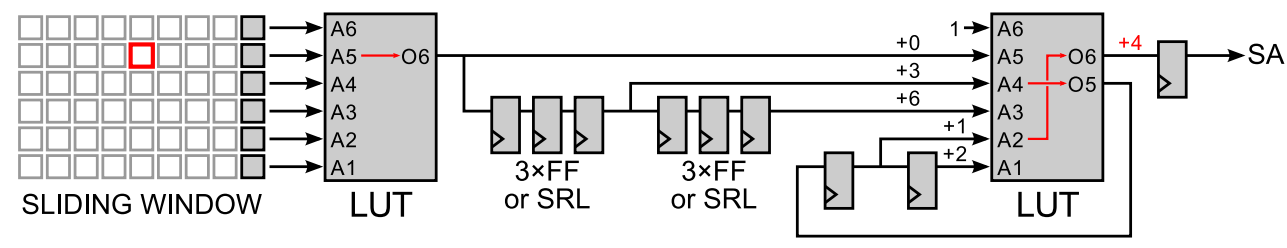

Figure 8-1: Possible implementation of a $6 \times 9$ window selector, with example configuration for a specific pixel marked in red.

Another aspect of the architecture that may be improved is the level of parameterization, focusing mostly on improving the system performance. One example of a system characteristic that may benefit from parameterization is how the PEs are internally arranged. As stated in $\$ 5.4 .3$, the 7 Series version of the SA uses a different spatial arrangement of stages 1 and 2 in order to adapt to the geometry of these FPGAs (see Figure 5-38 in that section). However, this arrangement has a negative impact in the maximum operating frequency. ${ }^{5}$ Permitting the modification of this arrangement via module parameters would allow easily adapting the design to different requirements of the application (frequency or resource usage).

Regarding the dataflow approach, it was stated that having two different modules for the evolution and the real-time stream operation is redundant and could be avoided by developing a strictly dataflow-based module library that can function both for the evolution stage and the real-time operation (including a stream-based fitness computing unit). In addition, these dataflowbased modules could be made dynamically reconfigurable (as shown in $\$ 5.4$.1, Figure 5-34), which would result in a highly flexible processing architecture.

Finally, it would be worthwhile studying and improving the fault tolerance capabilities of the system. First, the current design makes the input and output selector chains vulnerable points of failure; in order to make this design fully fault tolerant, redundancy techniques such as error-correcting codes (for the input selectors) and staggered TMR voters [Lee'01] (for the output selector)

\footnotetext{
${ }^{5} \mathrm{~A}$ timing analysis showed that this arrangement entails a reduction in the maximum operating frequency of about $18 \%$.
} 
may be used. In addition, for a truly fault tolerant design, the rest of the hardware system should be fault tolerant as well, using techniques such as TMR. Furthermore, the impact of faults over a parallelized SA system should be studied as well, since a fault affecting a single SA could be easily corrected by not using that SA or treating it differently to the rest. The learning by imitation technique proposed in [Gallego'13b] could be useful for this situation.

\subsubsection{Recofiguration methodology and tools}

Another area of the thesis where future work may be done is the one covering the partial reconfiguration methodology.

The partial bitstream extraction tool has several aspects that may need to be improved. Specifically, it may be a good idea to move to a higher level interpretation of the bitstream commands, since the tool currently assumes that all configuration data appears on a single contiguous block, whereas this may not always be the case: if configuration data is sent in multiple configuration packets, the tool should be able to combine them to form the overall bitstream. Another point of this tool that would need to be improved is the extension to a wider range of FPGAs, possibly by finding a way to automate the generation of FPGA definition files.

The DPR engine could also be improved in several ways. First, some parts of this project have shown the need to combine multiple PBSs into one (e.g., to configure dual-output PEs by combining two "basic configurations", one for each output, rather than storing all possible combinations beforehand). This would provide a higher flexibility for fine grain reconfiguration tasks. Second, it might be necessary to provide the ability to adapt to different PE layouts in case the PE sizes and positions are not constant across the design, with some regions having a different layout to others. And last, it may be useful to include readback capabilities in order to reconfigure regions that mix static and reconfigurable logic, or where the flip-flop state needs to be saved.

Finally, elaborating a routing tool for Vivado similar to DREAMS would allow extending the reconfiguration methodology to the interconnections rather than limiting it to the LUTs. Work in this topic is already being done [Zamacola'18], and will hopefully provide a framework to perform fine- and coarse-grain reconfigurable designs. 


\subsubsection{Applications and future projects}

Finally, an area of research that should be given some attention is the exploration of potential applications derived from the methodology described in this thesis, aside from the use cases already presented.

An application that could be implemented in the near future for demonstrative purposes is a real-time high-definition video processing application. This would extend the application described in $\$ 7.3$ to real-time video input rather than using a predefined sequence, on a development board that supports it such as the PYNQ-Z1. ${ }^{6}$ This implementation may be complemented by an experimental analysis of the maximum operating frequency on this platform: currently, pixel frequencies of up to $165 \mathrm{MHz}$ have been tested-enough to cover the demands of HDMI 1.2 and provide Full HD video $(1920 \times 1080)$ at 60 fps-; however, timing analysis showed that the SA can work at up to $300 \mathrm{MHz}$, although it is likely that this limit can be exceeded - a pixel frequency of $340 \mathrm{MHz}$ would be enough to cover all formats supported by HDMI 1.3, including WQXGA $(2560 \times 1600)$ at $60 \mathrm{fps}$ or $4 \mathrm{~K}$ UHD $(3480 \times 2160)$ at $30 \mathrm{fps}$.

It would also be worthwhile to explore possible applications that go beyond image processing, such as image classification, sound processing, multichannel EEG processing and classification, control of cyber-physical systems, etc. While image processing is a very useful use case from the academic point of view, since there are plenty of published use cases and metrics with which to compare and an immediate visual feedback of the quality of the results, EHW has plenty of data processing applications outside of it. This jump to different signal types will pose several challenges such as different representations and distributions of data, which may require modifications on the hardware architecture and EA.

Finally, porting this system to multiple platforms (different FPGA families, CPU, GPU...) would allow deploying heterogeneous distributed computing networks that accelerate the execution of an EA across multiple nodes and enabling the use of this system on platforms such as wireless sensor networks.

\footnotetext{
${ }^{6}$ This board has the same all-programmable SoC as the Zedboard-a Zynq 7020—and two HDMI video connectors, allowing the implementation of video filtering systems.
} 


\section{Bibliography}

[Amdahl'67] G. M. Amdahl, "Validity of the Single Processor Approach to Achieving Large Scale Computing Capabilities", in Proceedings of the April 18-20, 1967, Spring Joint Computer Conference, AFIPS '67 (Spring), pp. 483-485, ACM, New York, NY, USA, 1967, doi:10.1145/1465482.1465560. ${ }^{172}$

[ARM-IHI0022E] ARM, AMBA AXI and ACE Protocol Specification (ARM IHI 0022E). ${ }^{148}$

[ARM-IHI0051A] ARM, AMBA 4 AXI4-Stream Protocol Specification (ARM IHI O051A). ${ }^{149}$

[Bäck'96] T. Bäck, Evolutionary Algorithms in Theory and Practice, Oxford University Press, Oxford, UK, 1996, ISBN 0-19509971-0. ${ }^{19,47,50}$

[Barajas'17] R. Barajas, Análisis y optimización de algoritmos genéticos para el filtrado de imágenes mediante hardware evolutivo, B.Sc. thesis, Universidad Politécnica de Madrid, Feb. 2017, http://oa.upm.es/45666/. 162, 230, 233

[Bartolini'13] D. B. Bartolini, M. Carminati, F. Cancare, M. D. Santambrogio, D. Sciuto, "HERA Project's Holistic Evolutionary Framework", in 2013 IEEE International Symposium on Parallel Distributed Processing, Workshops and Phd Forum, pp. 231-238, May 2013, doi:10.1109/IPDPSW.2013.110. ${ }^{33}$

[Claus'10] C. Claus, R. Ahmed, F. Altenried, W. Stechele, Towards Rapid Dynamic Partial Reconfiguration in Video-Based Driver Assistance Systems, pp. 55-67, Springer Berlin Heidelberg, Berlin, Heidelberg, 2010, ISBN 978-3-642-12133-3, doi: 10.1007/978-3-642-12133-3_8. ${ }^{51,75,78}$

[Conejo'15] R. Conejo, Brain-computer interfaces: desarrollo de sistema de adquisición de datos, B.Sc. thesis, Universidad Politécnica de Madrid, Jul. 2015. ${ }^{230}$ 
[Correa'17] C. J. Correa, Adquisición y procesamiento multisensorial en FPGA para EEG, B.Sc. thesis, Universidad Politécnica de Madrid, Feb. 2017, http://oa.upm.es/45521/. ${ }^{230}$

[Dey'12] N. Dey, P. Nandi, N. Barman, D. Das, S. Chakraborty, "A Comparative Study between Moravec and Harris Corner Detection of Noisy Images Using Adaptive Wavelet Thresholding Technique", in International Journal of Engineering Research and Applications (IJERA), vol. 2, no. 1, pp. 599-606, Jan.-Feb. 2012, https://www.ijera.com/papers/Vol2_issue1/ CS21599606.pdf. ${ }^{210}$

[Dobai'13] R. Dobai, L. Sekanina, "Towards evolvable systems based on the Xilinx Zynq platform", in 2013 IEEE International Conference on Evolvable Systems (ICES), pp. 89-95, April 2013, doi:10.1109/ICES.2013.6613287. ${ }^{152}$

[Dobai'14] R. Dobai, K. Glette, J. Tørresen, L. Sekanina, "Evolutionary digital circuit design with fast candidate solution establishment in field programmable gate arrays", in Evolvable Systems (ICES), 2014 IEEE International Conference on, pp. 85-92, Dec 2014, doi:10.1109/ICES.2014.7008726. $33,76,114,129,138,160,164,170,171,177$

[Dobai'15] R. Dobai, L. Sekanina, "Low-Level Flexible Architecture with Hybrid Reconfiguration for Evolvable Hardware", in ACM Trans. Reconfigurable Technol. Syst., vol. 8, no. 3, pp. 20:120:24, May 2015, ISSN 1936-7406, doi:10.1145/2700414. ${ }^{33}$

[EIA/CEA-861-B] Electronic Industries Alliance, A DTV Profile for Uncompressed High Speed Digital Interfaces (EIA/CEA-861-B). ${ }^{158}$

[Fernández'14] V. Fernández, Modelado de hardware evolutivo sobre plataformas CUDA, B.Sc. thesis, Universidad Politécnica de Madrid, Sep. 2014. ${ }^{65,114,231}$

[Fogel'62] L. J. Fogel, "Autonomous automata", in Industrial Research, vol. 4, pp. $14-19,1962 .{ }^{20}$

[Fogel'96] D. B. Fogel, L. J. Fogel, "An introduction to evolutionary programming", in J.-M. Alliot, E. Lutton, E. Ronald, M. Schoenauer, D. Snyers, editors, Artificial Evolution, pp. 21-33, Springer Berlin Heidelberg, Berlin, Heidelberg, 1996, ISBN 978-3-540-49948-0, doi:10.1007/3-540-61108-8_28. ${ }^{20}$ 
[Gallego'12] Á. Gallego, Implementación de un sistema evolutivo escalable, B.Sc./M.Sc. final project, Universidad Politécnica de Madrid, Oct. 2012. ${ }^{143,188}$

[Gallego'13a] Á. Gallego, J. Mora, A. Otero, B. López, E. de la Torre, T. Riesgo, "A self-adaptive image processing application based on evolvable and scalable hardware", in $201323 \mathrm{rd}$ International Conference on Field programmable Logic and Applications (FPL), pp. 1-1, Sep. 2013, ISSN 1946-147X, doi: 10.1109/FPL.2013.6645631. Demo. ${ }^{228}$

[Gallego'13b] Á. Gallego, J. Mora, A. Otero, R. Salvador, E. de la Torre, T. Riesgo, "A Novel FPGA-based Evolvable Hardware System Based on Multiple Processing Arrays", in 2013 IEEE International Symposium on Parallel Distributed Processing, Workshops and Phd Forum, pp. 182-191, May 2013, doi: 10.1109/IPDPSW.2013.56. ${ }^{45,66,129,144,226,235}$

[Gallego'13c] Á. Gallego, J. Mora, A. Otero, E. de la Torre, T. Riesgo, "A scalable evolvable hardware processing array", in 2013 International Conference on Reconfigurable Computing and FPGAs (ReConFig), pp. 1-7, Dec 2013, ISSN 2325-6532, doi:10.1109/ReConFig.2013.6732266. $33,37,45,46,63,66,100,114,121,128,129,135,225$

[Gallego'14] Á. Gallego, Dynamically scalable evolvable hardware processing array, Master's thesis, Universidad Politécnica de Madrid, Mar. 2014, http://www.cei.upm.es/media/TFM/ Gallego_Angel_TFM_2014.pdf. ${ }^{3,51,144,145,150,196}$

[Garis'94] H. de Garis, "CAM-BRAIN Growing an Artificial Brain with a Million Neural Net Modules Inside a Trillion Cell Cellular Automata Machine", in Journal of the Society of Instrument and Control Engineers, vol. 33, no. 2, pp. 128-134, 1994, doi: 10.11499/sicejl1962.33.128. ${ }^{15}$

[Garis'02] H. de Garis, M. Korkin, "The CAM-Brain Machine (CBM): an FPGA-based hardware tool that evolves a 1000 neuronnet circuit module in seconds and updates a 75 million neuron artificial brain for real-time robot control", in Neurocomputing, vol. 42, no. 1, pp. 35-68, 2002, ISSN 0925-2312, doi:10.1016/S0925-2312(01)00593-8. Evolutionary neural systems. 25,32 
[Glette'07] K. Glette, J. Tørresen, M. Yasunaga, "An Online EHW Pattern Recognition System Applied to Face Image Recognition", in M. Giacobini, editor, Applications of Evolutionary Computing, pp. 271-280, Springer Berlin Heidelberg, Berlin, Heidelberg, Apr. 2007, ISBN 978-3-540-71805-5, doi:10.1007/978-3540-71805-5_30. ${ }^{33}$

[Glette'08] K. Glette, T. Gruber, P. Kaufmann, J. Tørresen, B. Sick, M. Platzner, "Comparing Evolvable Hardware to Conventional Classifiers for Electromyographic Prosthetic Hand Control", in Adaptive Hardware and Systems, 2008. AHS '08. NASA/ESA Conference on, pp. 32-39, June 2008, doi: 10.1109/AHS.2008.12. ${ }^{33,112}$

[Glette’09] K. Glette, J. Tørresen, M. Hovin, “Intermediate Level FPGA Reconfiguration for an Online EHW Pattern Recognition System", in Adaptive Hardware and Systems, 2009. AHS 2009. NASA/ESA Conference on, pp. 19-26, July 2009, doi: 10.1109/AHS.2009.46. ${ }^{33}$

[Glette'14] K. Glette, P. Kaufmann, "Lookup table partial reconfiguration for an evolvable hardware classifier system", in 2014 IEEE Congress on Evolutionary Computation (CEC), pp. 1706-1713, July 2014, ISSN 1089-778X, doi: 10.1109/CEC.2014.6900503. ${ }^{33}$

[Goldberg'89] D. E. Goldberg, Genetic Algorithms in Search, Optimization and Machine Learning, Addison-Wesley Longman Publishing Co., Inc., Boston, MA, USA, 1st ed., 1989, ISBN 0-20115767-5. ${ }^{20}$

[González'17] C. González, Filtrado mediante hardware evolutivo de imágenes de tamaño arbitrario sobre plataforma Zynq, B.Sc. thesis, Universidad Politécnica de Madrid, 2017, http://oa. upm.es/45517/. ${ }^{3,143,155,156,230}$

[Gustafson'88] J. L. Gustafson, "Reevaluating Amdahl's Law", in Commun. $A C M$, vol. 31, no. 5, pp. 532-533, May 1988, ISSN 0001-0782, doi:10.1145/42411.42415. ${ }^{172}$

[Hansen'11] S. G. Hansen, D. Koch, J. Tørresen, "High Speed Partial RunTime Reconfiguration Using Enhanced ICAP Hard Macro", 
in 2011 IEEE International Symposium on Parallel and Distributed Processing Workshops and Phd Forum, pp. 174-180, May 2011, ISSN 1530-2075, doi:10.1109/IPDPS.2011.139. $51,75,78$

[HDMI'05] HDMI Licensing, LLC, High-Definition Multimedia Interface Specification Version 1.2, 2005. ${ }^{157}$

[Higuchi'93] T. Higuchi, T. Niwa, T. Tanaka, H. Iba, H. de Garis, T. Furuya, "Evolving Hardware with Genetic Learning: A First Step Towards Building a Darwin Machine", in Proceedings of the Second International Conference on From Animals to Animats 2 : Simulation of Adaptive Behavior: Simulation of Adaptive Behavior, pp. 417-424, MIT Press, Cambridge, MA, USA, 1993, ISBN 0-262-63149-0, http://dl.acm.org/citation. cfm?id=171174.171223. ${ }^{32}$

[Higuchi'96] T. Higuchi, M. Iwata, I. Kajitani, H. Yamada, B. Manderick, Y. Hirao, M. Murakawa, S. Yoshizawa, T. Furuya, "Evolvable hardware with genetic learning", in 1996 IEEE International Symposium on Circuits and Systems. Circuits and Systems Connecting the World. ISCAS 96, vol. 4, pp. 29-32, May 1996, doi:10.1109/ISCAS.1996.541893. ${ }^{32}$

[Holland'75] J. H. Holland, Adaptation in natural and artificial systems: an introductory analysis with applications to biology, control, and artificial intelligence, University of Michigan Press, 1975, ISBN 9780472084609. ${ }^{20}$

[Iglesias'16] P. Iglesias, Brain-Computer Interfaces: Desarrollo de un sistema de identificación de estados mentales alfa, B.Sc. thesis, Universidad Politécnica de Madrid, Feb. 2016, http: //oa.upm.es/39564/. ${ }^{230}$

[Koza'92] J. R. Koza, Genetic Programming: On the Programming of Computers by Means of Natural Selection, MIT Press, Cambridge, MA, USA, 1992, ISBN 0-262-11170-5. ${ }^{20}$

[Kung'78] H. T. Kung, C. E. Leiserson, Systolic Arrays for (VLSI), CMU-CS, Carnegie-Mellon University, Department of Computer Science, 1978, http://www.dtic.mil/docs/citations/ ADA066060. 15, 26, 125 
[Kung'84] S.-Y. Kung, “On supercomputing with systolic/wavefront array processors", in Proceedings of the IEEE, vol. 72, no. 7, pp. 867-884, July 1984, ISSN 0018-9219, doi: 10.1109/PROC.1984.12944. ${ }^{27,135,137}$

[Lanza'13] D. Lanza, Implementación en hardware de un algoritmo evolutivo, B.Sc. thesis, Universidad Politécnica de Madrid, Sep. 2013. ${ }^{3,75,177}$

[Lee'01] S. Lee, I. Lee, "Staggered voting for TMR shift register chains in poly-Si TFT-LCDs", in Journal of Information Display, vol. 2, no. 2, pp. 22-26, 2001, doi: $10.1080 / 15980316.2001 .9651853 .{ }^{103,234}$

[Li'09] J. Li, "Evolvable Hardware Based Gray-level Image Enhancement", in 2009 NASA/ESA Conference on Adaptive Hardware and Systems, pp. 67-74, Jul. 2009, doi:10.1109/AHS.2009.12. 112

[Lie'09] W. Lie, W. Feng-yan, "Dynamic Partial Reconfiguration in FPGAs", in 2009 Third International Symposium on Intelligent Information Technology Application, vol. 2, pp. 445-448, Nov. 2009, doi:10.1109/IITA.2009.334. ${ }^{15}$

[Lim'04] D. Lim, M. Peattie, Two Flows for Partial Reconfiguration: Module Based or Small Bit Manipulations (XAPP290 v1.2), Xilinx, Inc., 9 Sep. 2004, https://web.archive. org/web/20071122065659/https://www.xilinx.com/ support/documentation/application_notes/xapp290.pdf. Description of bus macros only in v1.2 or earlier; latest version (v2.0) has removed all mentions of bus macros. ${ }^{30,43}$

[López'14] B. López, J. Mora, P. Mansanet, E. de la Torre, T. Riesgo, "Development of Brain-Computer Interfaces using Evolvable Hardware", in 2014 Conference on Design and Architectures for Signal and Image Processing (DASIP), pp. 1-1, IEEE, 2014, http://oa.upm.es/37019/. Poster presentation. ${ }^{3,227,229}$

[Lysaght'06] P. Lysaght, B. Blodget, J. Mason, J. Young, B. Bridgford, "Invited Paper: Enhanced Architectures, Design Methodologies and CAD Tools for Dynamic Reconfiguration of Xilinx FPGAs", in 2006 International Conference on Field 
Programmable Logic and Applications, pp. 1-6, Aug. 2006, ISSN 1946-147X, doi:10.1109/FPL.2006.311188. ${ }^{43}$

[Mann'47] H. B. Mann, D. R. Whitney, “On a Test of Whether one of Two Random Variables is Stochastically Larger than the Other", in The Annals of Mathematical Statistics, vol. 18, no. 1, pp. 5060, 03 1947, doi:10.1214/aoms/1177730491. ${ }^{7}$

[Mansanet'14] P. Mansanet, Biblioteca para modelado y simulación de alto nivel de sistemas evolutivos complejos implementados en hardware, B.Sc./M.Sc. final project, Universidad Politécnica de Madrid, Sep. 2014. ${ }^{231,232}$

[Mathworks] "Overview of Adaptive Filters and Applications", Mathworks, Inc., https://mathworks.com/help/dsp/ug/ overview-of-adaptive-filters-and-applications.html. ${ }^{14}$

[Mavroidis'12] I. Mavroidis, I. Mavroidis, I. Papaefstathiou, L. Lavagno, M. Lazarescu, E. de la Torre, F. Schäfer, "FASTCUDA: Open Source FPGA Accelerator \& Hardware-Software Codesign Toolset for CUDA Kernels", in 2012 15th Euromicro Conference on Digital System Design, pp. 343-348, Sep. 2012, doi: 10.1109/DSD.2012.58. ${ }^{231}$

[McCanny'82] J. V. McCanny, J. G. McWhirter, "Implementation of signal processing functions using 1-bit systolic arrays", in Electronics Letters, vol. 18, no. 6, pp. 241-243, March 1982, ISSN 00135194, doi:10.1049/el:19820166. ${ }^{41,135,137}$

[Merchant'10] S. G. Merchant, G. D. Peterson, "Evolvable Block-Based Neural Network Design for Applications in Dynamic Environments", in VLSI Design, vol. 2010, Article ID 251210, 2010, doi:10.1155/2010/251210. ${ }^{25,33}$

[Miller'99] J. F. Miller, “An Empirical Study of the Efficiency of Learning Boolean Functions Using a Cartesian Genetic Programming Approach", in Proceedings of the 1st Annual Conference on Genetic and Evolutionary Computation, vol. 2 of GECCO'99, pp. 1135-1142, Morgan Kaufmann Publishers Inc., San Francisco, CA, USA, 1999, ISBN 1-55860-611-4, http://dl. acm.org/citation.cfm?id=2934046.2934074. ${ }^{15,25,32}$

[Miller'11] J. F. Miller, editor, Cartesian Genetic Programming, Natural Computing Series, Springer-Verlag Berlin Heidelberg, 2011, 
ISBN 978-3-642-17310-3, doi:10.1007/978-3-642-17310-3. 49,112

[Mitchell'98] M. Mitchell, An Introduction to Genetic Algorithms, MIT Press, Cambridge, MA, USA, 1998, ISBN 0262631857. ${ }^{47,49}$

[Moon'01] S.-W. Moon, S.-G. Kong, "Block-based neural networks", in IEEE Transactions on Neural Networks, vol. 12, no. 2, pp. 307-317, Mar. 2001, ISSN 1045-9227, doi:10.1109/72.914525. $19,24,32,233$

[Mora'11] J. Mora, Implementación de hardware evolutivo en un array sistólico mediante reconfiguración parcial, B.Sc./M.Sc. final project, Universidad Politécnica de Madrid, Oct. 2011, http: //oa.upm.es/48786/. 2, 10,37, 51

[Mora'12] J. Mora, "Self-healing evolvable hardware architectures for image processing applications", Mar. 2012, http://www. cei.upm.es/media/seminarios/2012/Presentations/4_4_ Seminario_CEI_2012_SelfHealing.pdf. Oral presentation. ${ }^{228}$

[Mora'13a] J. Mora, Noise-agnostic self-adaptive evolvable hardware for real time video filtering applications, Master's thesis, Universidad Politécnica de Madrid, Sep. 2013, http://oa. upm.es/32194/. 2, 180, 181

[Mora'13b] J. Mora, Á. Gallego, A. Otero, E. de la Torre, T. Riesgo, "Noise-agnostic adaptive image filtering without training references on an evolvable hardware platform", in Design and Architectures for Signal and Image Processing (DASIP), 2013 Conference on, pp. 182-189, Oct 2013, http://ieeexplore.ieee. org/document/6661538/. ${ }^{2}, 33,58,135,181,226$

[Mora'13c] J. Mora, Á. Gallego, A. Otero, E. de la Torre, T. Riesgo, “A noiseagnostic self-adaptive image processing application based on evolvable hardware", in 2013 Conference on Design and Architectures for Signal and Image Processing, pp. 351-352, Oct. 2013, https://ieeexplore.ieee.org/document/6661571/. Demo. ${ }^{228}$

[Mora'14] J. Mora, Á. Gallego, A. Otero, E. de la Torre, T. Riesgo, "Increased fault tolerance in evolvable hardware through 
automatic upscaling", in Conference on Design and Architectures for Signal and Image Processing (DASIP 2014), pp. 1-1, 2014, http://oa.upm.es/37142/. Demo. ${ }^{2,64,187,188,227}$

[Mora'15]

J. Mora, A. Otero, E. de la Torre, T. Riesgo, "Fast and compact evolvable systolic arrays on dynamically reconfigurable FPGAs", in Reconfigurable Communication-centric Systemson-Chip (ReCoSoC), 2015 10th International Symposium on, pp. 1-7, June 2015, doi:10.1109/ReCoSoC.2015.7238087. $33,116,129,160,172,225$

[Mora'18a] J. Mora, R. Salvador, E. de la Torre, "On the scalability of evolvable hardware architectures: comparison of systolic array and Cartesian genetic programming", in Genetic Programming and Evolvable Machines, Oct. 2018, ISSN 15737632, doi:10.1007/s10710-018-9340-5. ${ }^{113,224}$

[Mora'18b] J. Mora, E. de la Torre, "Accelerating the evolution of a systolic array-based evolvable hardware system", in Microprocessors and Microsystems, vol. 56, pp. 144-156, Feb. 2018, ISSN 01419331, doi:10.1016/j.micpro.2017.12.001. ${ }^{33,129,168,224}$

[Neuendorffer'15] S. Neuendorffer, T. Li, D. Wang, Accelerating OpenCV Applications with Zynq-7000 All Programmable SoC using Vivado HLS Video Libraries (XAPP1167 v3.0), Xilinx, Inc., 24 Jun. 2015, https://www.xilinx.com/support/ documentation/application_notes/xapp1167.pdf. 144, 147, 211

[Niño'15] A. Niño, Análisis cuantitativo de filtros evolutivos sobre imágenes con ruido realista, B.Sc./ M.Sc. final project, Universidad Politécnica de Madrid, Apr. 2015. 3, 65, 114, 199

[Nurvitadhi'17] E. Nurvitadhi, G. Venkatesh, J. Sim, D. Marr, R. Huang, J. Ong Gee Hock, Y. T. Liew, K. Srivatsan, D. Moss, S. Subhaschandra, G. Boudoukh, "Can FPGAs Beat GPUs in Accelerating Next-Generation Deep Neural Networks?", in Proceedings of the 2017 ACM/SIGDA International Symposium on FieldProgrammable Gate Arrays, FPGA '17, pp. 5-14, ACM, New York, NY, USA, 2017, ISBN 978-1-4503-4354-1, doi: $10.1145 / 3020078.3021740 .{ }^{15}$

[OpenCV] “About”, OpenCV library, https://opencv.org/about.html. ${ }^{211}$ 
[Otero'10] A. Otero, A. Morales-Cas, J. Portilla, E. de la Torre, T. Riesgo, "A Modular Peripheral to Support Self-Reconfiguration in SoCs", in 2010 13th Euromicro Conference on Digital System Design: Architectures, Methods and Tools, pp. 88-95, Sept 2010, doi:10.1109/DSD.2010.100. ${ }^{42,45,75,76}$

[Otero'11a] A. Otero, R. Salvador, J. Mora, E. de la Torre, T. Riesgo, L. Sekanina, "2D Reconfigurable Systolic Core Architecture for Evolvable Systems", in Proceedings of the XXVI Conference on Design of Circuits and Integrated Systems (DCIS 2011), Nov. 2011. ${ }^{227}$

[Otero'11b] A. Otero, R. Salvador, J. Mora, E. de la Torre, T. Riesgo, L. Sekanina, "A fast Reconfigurable 2D HW core architecture on FPGAs for evolvable Self-Adaptive Systems", in Adaptive Hardware and Systems (AHS), 2011 NASA/ESA Conference on, pp. 336-343, June 2011, doi:10.1109/AHS.2011.5963956. $2,33,227$

[Otero'12a] A. Otero, J. Mora, R. Salvador, Á. Gallego, E. de la Torre, L. Sekanina, "Evolvable hardware FPGA-based platform for autonomous fault-tolerant systems", in 2012 Design, Automation \& Test in Europe (DATE), University Booth, Dresden, Germany, Mar. 2012. Demo. ${ }^{2,228}$

[Otero'12b] A. Otero, J. Mora, R. Salvador, Á. Gallego, E. de la Torre, L. Sekanina, "Evolvable hardware FPGA-based platform for autonomous fault-tolerant systems", in 2012 International Conference on ReConFigurable Computing and FPGAs (ReConFig), Demo Night, Cancun (Mexico), Dec. 2012. Demo. ${ }^{228}$

[Otero'12c] A. Otero, E. de la Torre, T. Riesgo, "Dreams: A tool for the design of dynamically reconfigurable embedded and modular systems", in 2012 International Conference on Reconfigurable Computing and FPGAs, pp. 1-8, Dec 2012, ISSN 2325-6532, doi:10.1109/ReConFig.2012.6416740. ${ }^{46,63}$

[Otero'14] A. Otero, Run-Time Scalable Hardware for Reconfigurable Systems, Ph.D. thesis, Universidad Politécnica de Madrid, 2014, http://oa.upm.es/22584/. 2, 10, 37

[Polop'14] F. J. Polop, Implementación de filtros de eliminación de moteado de imágenes mediante hardware evolutivo iterativo, 
B.Sc. thesis, Universidad Politécnica de Madrid, Sep. 2014. 3,231

[Rashmi'13] Rashmi, M. Kumar, R. Saxena, "Algorithm and Technique on Various Edge Detection: A Survey", in Signal \& Image Processing : An International Journal, vol. 4, no. 3, pp. 65-75, Jun. 2013, doi:10.5121/sipij.2013.4306. ${ }^{210}$

[Rechenberg'65] I. Rechenberg, "Cybernetic solution path of an experimental problem", in Royal Aircraft Establishment Translation No. 1122, B. F. Toms, Trans., Ministry of Aviation, Royal Aircraft Establishment, Farnborough Hants, Aug. 1965. ${ }^{19}$

[Rodríguez'18] A. Rodríguez, J. Valverde, J. Portilla, A. Otero, T. Riesgo, E. de la Torre, "FPGA-Based High-Performance Embedded Systems for Adaptive Edge Computing in Cyber-Physical Systems: The ARTICo ${ }^{3}$ Framework", in Sensors, vol. 18, no. 6, 2018, ISSN 1424-8220, doi:10.3390/s18061877. ${ }^{229}$

[Rojas'15] P. Sánchez de Rojas, Ejecución de aplicaciones multihilo sobre sistemas en FPGA con aceleradores hardware descritos en CUDA, B.Sc./M.Sc. final project, Universidad Politécnica de Madrid, Sep. 2015, http://oa.upm.es/43483/. ${ }^{230}$

[Rosenblatt'62] F. Rosenblatt, Principles of neurodynamics: perceptrons and the theory ofbrain mechanisms, Report (Cornell Aeronautical Laboratory), Spartan Books, 1962. ${ }^{23}$

[Rosten'06] E. Rosten, T. Drummond, “Machine Learning for High-Speed Corner Detection", in Computer Vision - ECCV 2006, pp. 430443, Springer Berlin Heidelberg, Berlin, Heidelberg, 2006, ISBN 978-3-540-33833-8, doi:10.1007/11744023_34. ${ }^{210,211}$

[Saeidi'05] M. Saeidi, S. A. Motamedi, A. Behrad, B. Saeidi, R. Saeidi, R. Saeidi, "Noise reduction of consecutive images using a new adaptive weighted averaging filter", in IEEE Workshop on Signal Processing Systems Design and Implementation, 2005., pp. 455-460, Nov 2005, ISSN 2162-3562, doi: 10.1109/SIPS.2005.1579912. ${ }^{52,184,185,187}$

[Salvador'11a] R. Salvador, A. Otero, J. Mora, E. de la Torre, T. Riesgo, L. Sekanina, "Evolvable 2D computing matrix model for intrinsic evolution in commercial FPGAs with native reconfiguration support", in Adaptive Hardware and Systems (AHS), 
2011 NASA/ESA Conference on, pp. 184-191, June 2011, doi: 10.1109/AHS.2011.5963934. ${ }^{27,33,41,96,114,135,136,199,203,227}$

[Salvador'11b] R. Salvador, A. Otero, J. Mora, E. de la Torre, L. Sekanina, T. Riesgo, "Fault Tolerance Analysis and Self-Healing Strategy of Autonomous, Evolvable Hardware Systems", in 2011 International Conference on Reconfigurable Computing and FPGAs, pp. 164-169, Nov 2011, ISSN 2325-6532, doi: 10.1109/ReConFig.2011.37. ${ }^{2,15,33,37,63,226}$

[Salvador'12] R. Salvador, A. Otero, J. Mora, E. de la Torre, T. Riesgo, L. Sekanina, "Implementation techniques for evolvable HW systems: virtual VS. dynamic reconfiguration", in 22nd International Conference on Field Programmable Logic and Applications (FPL), pp. 547-550, Aug. 2012, ISSN 1946-147X, doi:10.1109/FPL.2012.6339376. ${ }^{226}$

[Salvador'13] R. Salvador, A. Otero, J. Mora, E. de la Torre, T. Riesgo, L. Sekanina, "Self-Reconfigurable Evolvable Hardware System for Adaptive Image Processing", in IEEE Transactions on Computers, vol. 62, no. 8, pp. 1481-1493, Aug 2013, ISSN 0018-9340, doi:10.1109/TC.2013.78. 47, 57, 59, 60, 135, 160, 224

[Salvador'15] R. Salvador, Parametric and structural self-adaptation of embedded systems using evolvable hardware, Ph.D. thesis, Universidad Politécnica de Madrid, 2015, http://oa.upm.es/ $39354 / .^{2,10,37}$

[Schwefel'77] H.-P. Schwefel, Numerische Optimierung von ComputerModellen mittels der Evolutionsstrategie, Ph.D. thesis, Universität Dortmund, 1977, doi:10.1007/978-3-0348-5927-1, https://www.researchgate.net/publication/248568763. ${ }^{19,47}$

[Schwefel'93] H.-P. Schwefel, Evolution and Optimum Seeking: The Sixth Generation, John Wiley \& Sons, Inc., New York, NY, USA, 1993, ISBN 0471571482. ${ }^{19}$

[Sekanina'03] L. Sekanina, "Virtual Reconfigurable Circuits for RealWorld Applications of Evolvable Hardware", in Lecture Notes in Computer Science, vol. 2003, no. 2606, pp. 186-197, 2003, ISSN 0302-9743, http://www.fit.vutbr.cz/research/ view_pub.php?id=7150. ${ }^{29,32,41,114,135}$ 
[Sekanina'04] L. Sekanina, Evolvable Components: From Theory to Hardware Implementations, Natural Computing Series, SpringerVerlag Berlin Heidelberg, 2004, ISBN 978-3-540-40377-7, doi: 10.1007/978-3-642-18609-7. 127, 199, 203, 233

[Sekanina'13] L. Sekanina, Z. Vašíček, "Approximate circuit design by means of evolvable hardware", in Evolvable Systems (ICES), 2013 IEEE International Conference on, pp. 21-28, April 2013, doi:10.1109/ICES.2013.6613278. ${ }^{49}$

[Shayani'13] H. Shayani, A Practical Investigation into Achieving BioPlausibility in Evo-Devo Neural Microcircuits Feasible in an FPGA, Ph.D. thesis, University College London, 2013, http: //discovery.ucl.ac.uk/1420124/. ${ }^{92}$

[Student'08] Student, “The Probable Error of a Mean”, in Biometrika, vol. 6, no. 1, pp. 1-25, 1908, ISSN 00063444, doi: $10.2307 / 2331554$. $^{7,162}$

[Sudholt'15] D. Sudholt, Parallel Evolutionary Algorithms, pp. 929-959, Springer Berlin Heidelberg, Berlin, Heidelberg, 2015, ISBN 978-3-662-43505-2, doi:10.1007/978-3-662-43505-2_46. ${ }^{167}$

[Thompson'95] A. Thompson, "Evolving fault tolerant systems", in First International Conference on Genetic Algorithms in Engineering Systems: Innovations and Applications, pp. 524-529, Sep. 1995, ISSN 0537-9989, doi:10.1049/cp:19951102. ${ }^{15}$

[Thompson'97] A. Thompson, “An evolved circuit, intrinsic in silicon, entwined with physics", in T. Higuchi, M. Iwata, W. Liu, editors, Evolvable Systems: From Biology to Hardware, pp. 390-405, Springer Berlin Heidelberg, Berlin, Heidelberg, 1997, ISBN 978-3-540-69204-1, doi:10.1007/3-540-631739_61. 22, 28, 32

[Tørresen’00] J. Tørresen, “Scalable evolvable hardware applied to road image recognition", in Proceedings. The Second NASA/DoD Workshop on Evolvable Hardware, pp. 245-252, Jul. 2000, doi: 10.1109/EH.2000.869362. ${ }^{32}$

[Tyrrell'01] A. M. Tyrrell, G. Hollingworth, S. L. Smith, "Evolutionary strategies and intrinsic fault tolerance", in Proceedings Third NASA/DoD Workshop on Evolvable Hardware. EH-2001, pp. 98-106, Jul. 2001, doi:10.1109/EH.2001.937951. ${ }^{15}$ 
[Upegui'06] A. Upegui, E. Sanchez, "Evolving Hardware with Selfreconfigurable connectivity in Xilinx FPGAs", in First NASA/ESA Conference on Adaptive Hardware and Systems (AHS'06), pp. 153-162, Jun. 2006, doi:10.1109/AHS.2006.38. 22,32

[Valverde'14] J. Valverde, A. Rodríguez, J. Mora, C. Castañares, J. Portilla, E. de la Torre, T. Riesgo, "A Dynamically Adaptable Image Processing Application Trading Off Between High Performance, Consumption and Dependability in Real Time", 2014, http://oa.upm.es/37015/. Demo. ${ }^{227}$

[Vašíček'07a] Z. Vašíček, L. Sekanina, "Evaluation of a New Platform For Image Filter Evolution", in Second NASA/ESA Conference on Adaptive Hardware and Systems (AHS 2007), pp. 577-586, Aug 2007, doi:10.1109/AHS.2007.49. 52, 160

[Vašíček'07b] Z. Vašíček, L. Sekanina, "An Evolvable Hardware System in Xilinx Virtex II Pro FPGA", in International Journal of Innovative Computing and Applications, vol. 1, no. 1, pp. 6373, 2007, ISSN 1751-648X, doi:10.1504/IJICA.2007.013402. $32,41,112$

[Vašíček'09] Z. Vašíček, M. Bidlo, L. Sekanina, J. Tørresen, K. Glette, M. Furuholmen, "Evolution of Impulse Bursts Noise Filters", in 2009 NASA/ESA Conference on Adaptive Hardware and Systems, pp. 27-34, July 2009, doi:10.1109/AHS.2009.33. 39,234

[Vassilev'99] V. K. Vassilev, J. F. Miller, T. C. Fogarty, "On the nature of two-bit multiplier landscapes", in Proceedings of the First NASA/DoD Workshop on Evolvable Hardware, pp. 36-45, 1999, doi:10.1109/EH.1999.785433. ${ }^{233}$

[Veljković'13] F. Veljković, J. Mora, T. Riesgo, L. Berrojo, R. Regada, Á. Álvaro, E. de la Torre, "Prospection of Reconfiguration Capabilities using Space Qualified SRAM-based FPGAs for a Satellite Communications Application", in 31st AIAA International Communications Satellite Systems Conference, International Communications Satellite Systems Conferences (ICSSC), American Institute of Aeronautics and Astronautics, Oct. 2013, doi:10.2514/6.2013-5683. ${ }^{15,225}$ 
[Wasserman'89] P. D. Wasserman, Neural Computing: Theory and Practice, Van Nostrand Reinhold Co., New York, NY, USA, 1989, ISBN 0-442-20743-3. ${ }^{22}$

[Wolpert'97] D. H. Wolpert, W. G. Macready, "No free lunch theorems for optimization", in IEEE Transactions on Evolutionary Computation, vol. 1, no. 1, pp. 67-82, Apr. 1997, ISSN 1089778X, doi:10.1109/4235.585893. ${ }^{17}$

[Xilinx-DS100] Xilinx, Inc., Virtex-5 Family Overview (DS100), https: //www.xilinx.com/support/documentation/data_sheets/ ds100.pdf. ${ }^{9,143}$

[Xilinx-DS202] Xilinx, Inc., Virtex-5 FPGA Data Sheet: DC and Switching Characteristics, https://www.xilinx.com/support/ documentation/data_sheets/ds202.pdf. ${ }^{131,138}$

[Xilinx-DS586] Xilinx, Inc., LogiCORE IP XPS HWICAP (DS586), https://www.xilinx.com/support/documentation/ip_ documentation/xps_hwicap.pdf. ${ }^{41}$

[Xilinx-PG085] Xilinx, Inc., AXI4-Stream Infrastructure IP Suite v2.2 (PG085), https://www.xilinx.com/support/documentation/ ip_documentation/axis_infrastructure_ip_suite/v1_1/ pg085-axi4stream-infrastructure.pdf. ${ }^{150,212}$

[Xilinx-PG231] Xilinx, Inc., Video Processing Subsystem v1.0 (PG231), https://www.xilinx.com/support/documentation/ip_ documentation/v_proc_ss/v1_0/pg231-v-proc-ss.pdf. 144,147

[Xilinx-UG190] Xilinx, Inc., Virtex-5 FPGA User Guide (UG190), https://www.xilinx.com/support/documentation/user_ guides/ug190.pdf. ${ }^{89,90,91,115}$

[Xilinx-UG191] Xilinx, Inc., Virtex-5 FPGA Configuration User Guide (UG191), https://www.xilinx.com/support/documentation/ user_guides/ug191.pdf. ${ }^{79,89,92}$

[Xilinx-UG470] Xilinx, Inc., 7 Series FPGAs Configuration User Guide (UG470), https://www.xilinx.com/support/documentation/ user_guides/ug470_7Series_Config.pdf. ${ }^{94}$ 
[Xilinx-UG585] Xilinx, Inc., Zynq-7000 All Programmable SoC (UG585), https://www.xilinx.com/support/documentation/user_ guides/ug585-Zynq-7000-TRM.pdf. ${ }^{152}$

[Xilinx-UG621] Xilinx, Inc., Virtex-5 Libraries Guide for HDL Designs (UG621), https://www.xilinx.com/support/documentation/ sw_manuals/xilinx14_7/virtex5_hdl.pdf. ${ }^{90,92}$

[Xilinx-UG625] Xilinx, Inc., Constraints Guide (UG625), https://www.xilinx. com/support/documentation/sw_manuals/xilinx14_7/cgd. pdf. ${ }^{89,104,106}$

[Xilinx-UG761] Xilinx, Inc., AXI Reference Guide (UG761), https://www. xilinx.com/support/documentation/ip_documentation/ ug761_axi_reference_guide.pdf. ${ }^{147}$

[Xilinx-UG904] Xilinx, Inc., Vivado Design Suite User Guide: Implementation (UG904), https://www.xilinx.com/support/documentation/ sw_manuals/xilinx2016_4/ug904-vivado-implementation. pdf. ${ }^{91,} 99$

[Xilinx-UG934] Xilinx, Inc., AXI4-Stream Video IP and System Design Guide (UG934), https://www.xilinx.com/support/documentation/ ip_documentation/axi_videoip/v1_0/ug934_axi_videoIP. pdf. ${ }^{149}$

[Xilinx-XUPV5] "Xilinx University Program XUPV5-LX110T Development System", Xilinx, Inc., https://www.xilinx.com/univ/ xupv5-lx110t.htm. ${ }^{38}$

[Xilinx'12] "Vivado - Are Spartan-6, Virtex-6 and older devices supported in the Vivado design tools?", 2012, https://www.xilinx. com/support/answers/53109.html. ${ }^{9,152}$

[Xilinx'14] "Vivado Constraints - LOC constraint does not support ranges in Vivado", Xilinx Answer Records, 2014, https:/ /www. xilinx.com/support/answers/52562.html. ${ }^{106}$

[Yao'99] X. Yao, T. Higuchi, "Promises and challenges of evolvable hardware", in IEEE Transactions on Systems, Man, and Cybernetics, Part C (Applications and Reviews), vol. 29, no. 1, pp. 87-97, Feb. 1999, ISSN 1094-6977, doi:10.1109/5326.740672. 15 
[Zamacola'18] R. Zamacola, A. García, J. Mora, A. Otero, E. de la Torre, "IMPRESS: Automated Tool for the Implementation of Highly Flexible Partial Reconfigurable Systems with Xilinx Vivado", in 2018 International Conference on Reconfigurable Computing and FPGAs (ReConFig), Dec. 2018, ISSN 23256532. $73,225,229,235$

[Zhou'98] X. Zhou, W. G. Wee, “Adaptive order statistic filters for noise characterization and suppression without a noise-free reference", in Communications, 1998. ICC 98. Conference Record. 1998 IEEE International Conference on, vol. 3, pp. 1774-1778, Jun 1998, doi:10.1109/ICC.1998.683134. ${ }^{52,181,182}$ 